\title{
AN INVESTIGATION OF DAMAGE ARRESTMENT DEVICES APPLICATION WITH FASTENER/HOLE INTERACTION
}

\author{
A Thesis \\ Presented to the \\ Aerospace Engineering Department \\ California Polytechnic State University, San Luis Obispo \\ In Partial Fulfillment of Requirements for the \\ Degree of Master of Science in Aerospace Engineering
}

By

Richard Vincent Santos Balatbat

September 2010 
(C) 2010

Richard Vincent Santos Balatbat All Rights Reserved 
Title:

Author:

Date Submitted:

COMMITTEE CHAIR:

COMMITTEE MEMBER:

COMMITTEE MEMBER:

COMMITTEE MEMBER:

COMMITTEE MEMBER:
An Investigation of Damage Arrestment Devices Application with Fastener/Hole Interaction

Richard Vincent Santos Balatbat

September 2010

Faysal Kolkailah, Ph.D., P.E.

Eltahry Elghandour, Ph.D.

Eric Mehiel, Ph.D.

Susan Opava, Ph.D.

Eric Kasper, Ph.D., P.E. 


\begin{abstract}
$\underline{\text { Abstract }}$
An Investigation of Damage Arrestment Devices Application with Fastener/Hole Interaction

by

Richard Vincent Santos Balatbat

This thesis presents a parametric study on the effects of how damage arrestment devices application interacts with a fastener in a composite sandwich panel. The primary objective of the damage arrestment device was to prevent the failure of the composite face sheet, such as crack propagation, around the hole/fastener joint. The damage arrestment devices are made of composite strips that are inserted under the face sheet to increase the overall structural strength of the panel and to prevent the propagation of failure along the hole. This was supposed to be a quicker and stronger alternative to potted inserts for composite sandwich panels for designer. The manufacturing curing cycle of the composite sandwich specimens has been carried out by using a Tetrahedron Composite Air Press. The press has been used to fabricate composite sandwich panels by applying constant pressure and variable heat to create panels with dimensions of 5 ” $\mathrm{x}$ 2” x .552”. The panels were stacked using a polyurethane foam, Last-A-Foam FR-6710 with two layers of a carbon-fiber/epoxy weave, LTM45, on both sides of the foam. The specimens were loaded under a compressive strain of $0.5 \mathrm{~mm} / \mathrm{min}$. The damage arrestment devices' thickness was varied and tested under both monotonic and fatigue loading.

The experimental results indicate that as the thickness of the device increased the overall strength of the part increased at a parabolic curve with it topping at a thickness of 0.065 ” and a strength increase of $109 \%$. Under fatigue loading, a control group test case and damage arrestment device configuration case was tested. The experimental results indicate that both cases have similar fatigue trends but shows that the damage arrestment specimens are stronger due to the increase of structural strength.

The experimental results were compared with numerical results or Finite Element Model. The results showed that numerical results can capture the linear or elastic portion of the experimental results having identical Elastic Modulus values. The models do differ in the maximum displacement of the specimen and the failure mode around the hole of the composite sandwich panel. The discrepancy in displacement and the failure mode was attributed to inaccurate loading on the hole of the composite sandwich panel and non-linear modeling of the solution. The correlation between the FEM and the experimental data was good enough in predicting the trends of the composite sandwich panels.
\end{abstract}




\section{$\underline{\text { Acknowledgement }}$}

I would like to first thank my thesis advisors, Dr. Faysal Kolkailah, Dr. Eltahry

Elghandour and Dr. Eric Kasper for providing me with the opportunity to work on such a project. I am sincerely grateful for their understanding, support, and dedicated assistance at all stages of this project. I would like to especially thank Dr. Elghandour for being in the lab in his off hours helping on all the experimental testing.

Thanks to all my family and friends who supported and believed in me from the beginning of my college career. Without their love, guidance, and support, I would not be the person who I am today. Thanks to my father and mother who inspired and motivated me to follow my dreams and were there to support all my decisions in life. Thanks to my brother who was there to support and believe in his older brother. Thanks to my friends and roommates for being there for help when I needed it.

I would also like to thank the Aerospace Department, the Aerospace Student Fee Committee, and C3RP for their funding and support of this project. Without their assistance, the materials and the lab upgrades would not be possible to conduct the research.

In addition, I would also like to thank Mr. Cody Thompson for his assistance in the lab and also letting me use his machine shop and tools to work on the research. Thanks to Michael Jacobson and Thomas Woo for giving me advice on the research and helping me build the test apparatus. Thanks to Danny Nunes and Matt Ales for helping me machine out the foam for the experimental testing. Thanks to Dominic Surano, Tony Tran, Ahmad Amini, and Jenny Shatts on helping me out in the lab during the research.

Finally, I would like to thank God for giving me the opportunity in life to succeed. 


\section{Table of Contents}

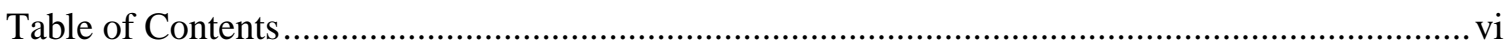

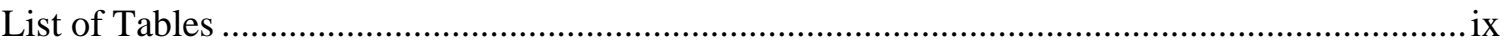

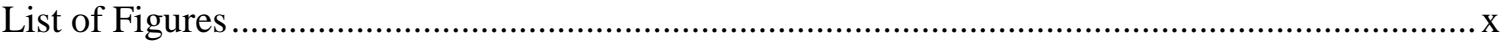

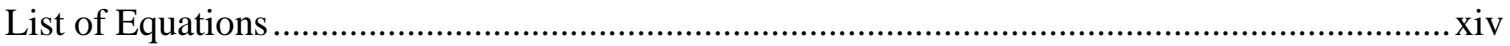

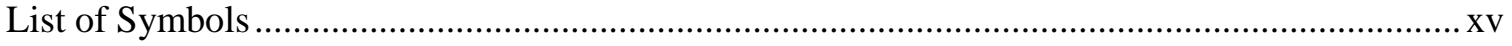

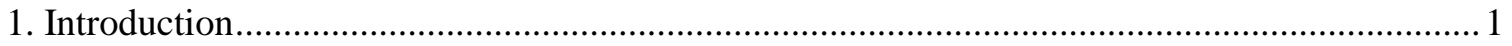

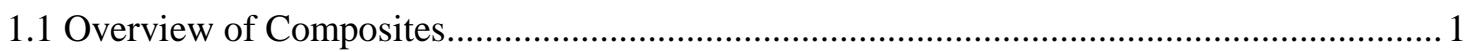

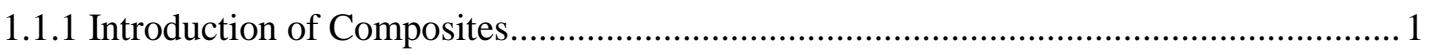

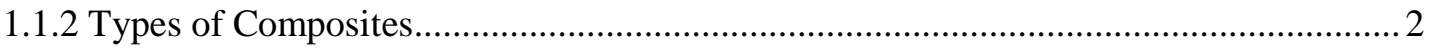

1.1.3 Advantages and Disadvantages of Composites............................................................. 4

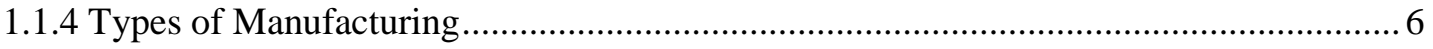

1.1.5 Composite Structures .............................................................................................. 9

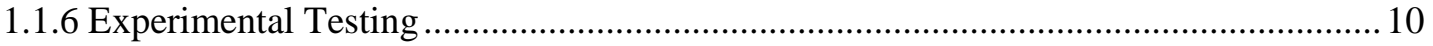

1.2 Application of Sandwich Composite Structures/Problems .................................................11

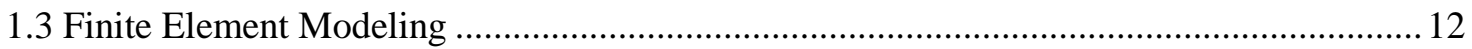

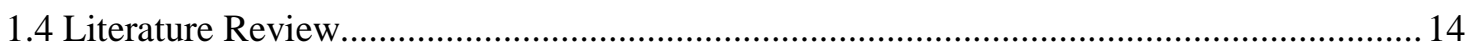

1.4.1 Composite Sandwiches with through holes with a shear key ..................................... 14

1.4.2 Fastener Failure Interaction of Composite Sandwich Panels......................................... 15

1.4.3 Monotonic and Dynamic Loading of the Composite Sandwich ................................... 17

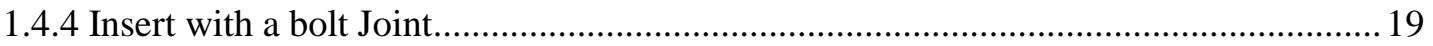

1.4.5 Finite Element Models of Composite/Fastener Interactions ......................................... 20

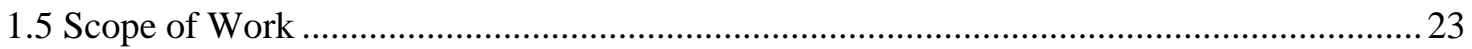

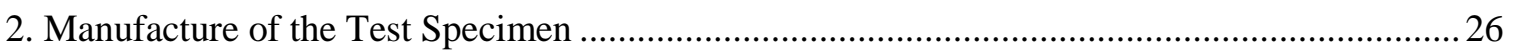

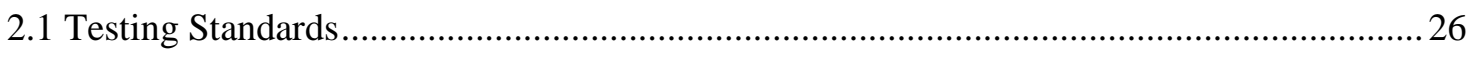

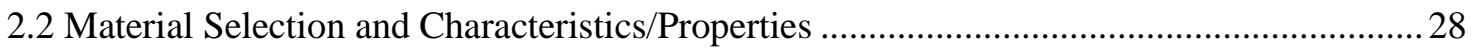

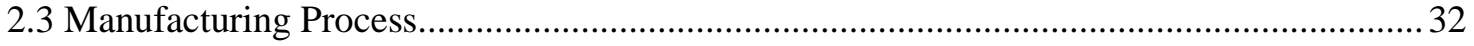

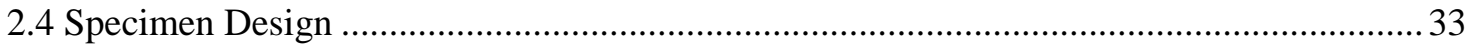

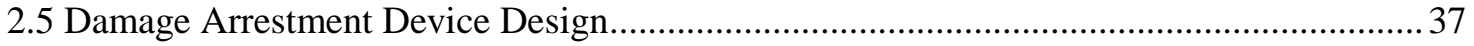

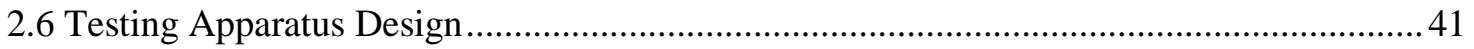

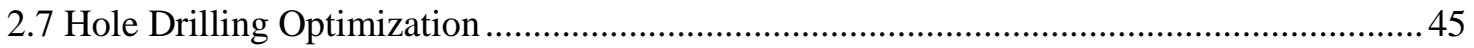




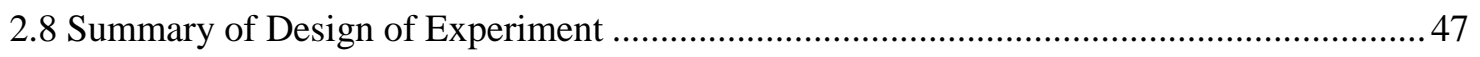

3. Manufacture Process and Prepping Procedure............................................................................. 49

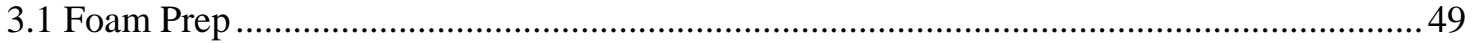

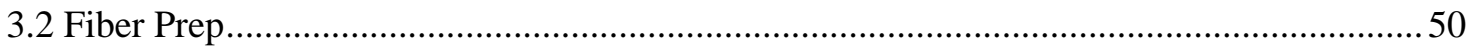

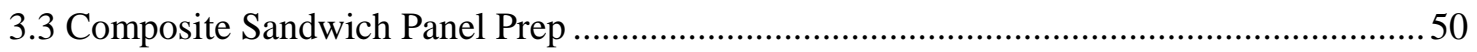

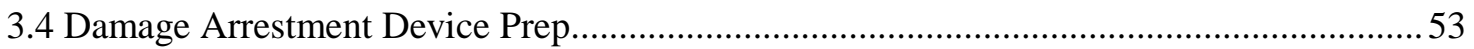

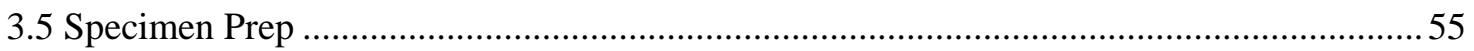

3.6 Testing Procedure of Volume Fraction of Face Sheet ..................................................... 59

3.7 Specimen Preparation for Material Properties Testing ......................................................... 61

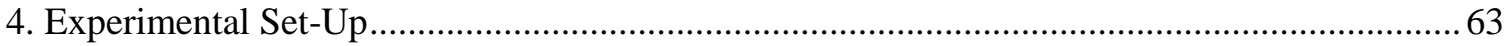

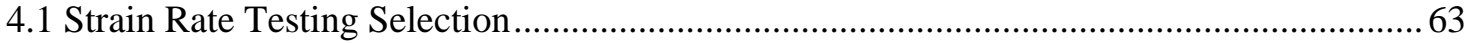

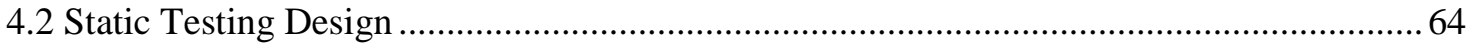

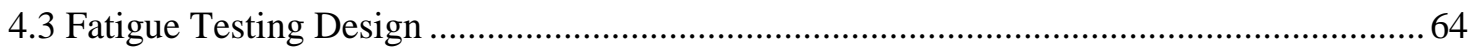

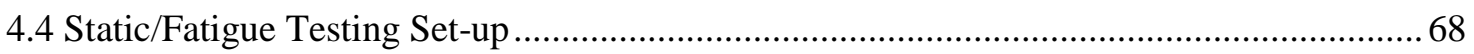

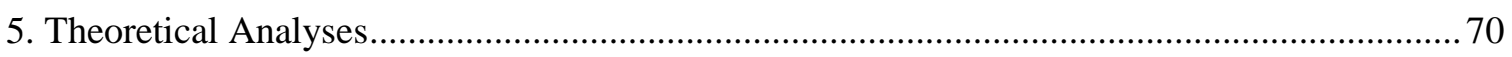

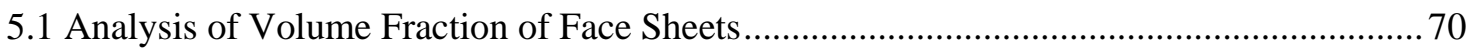

5.2 Analysis of Elastic Modulus of Composite Sandwich Panel ............................................... 72

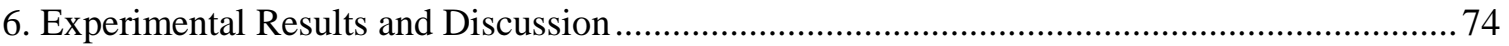

6.1 Mechanical Characteristics of Material/Specimen................................................................. 74

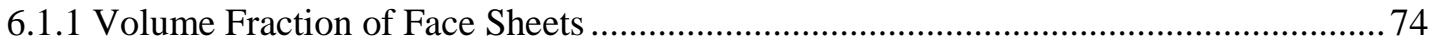

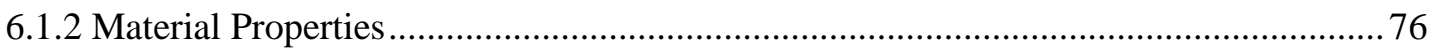

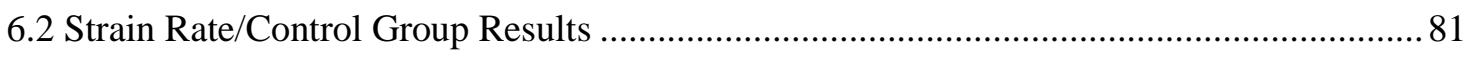

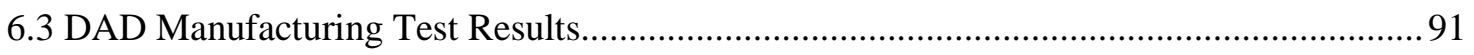

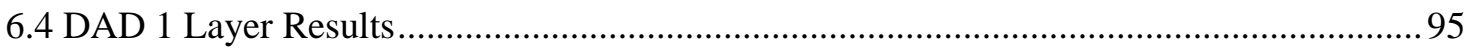

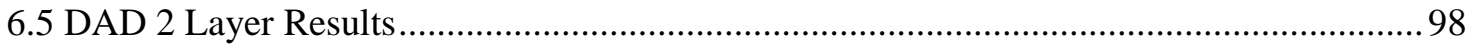

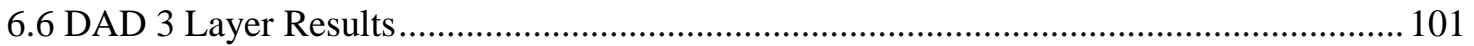

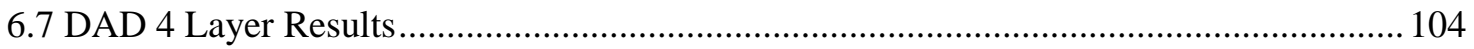

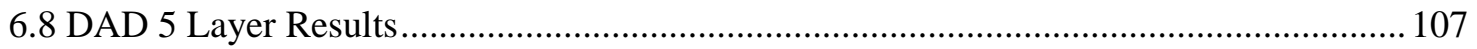

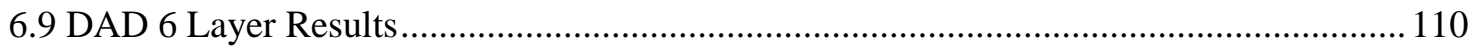

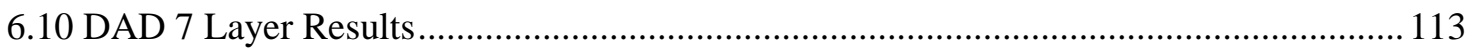

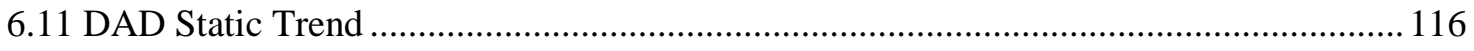

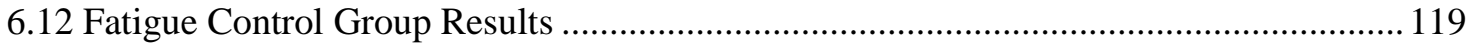

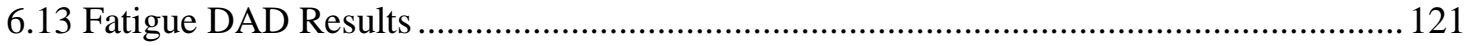




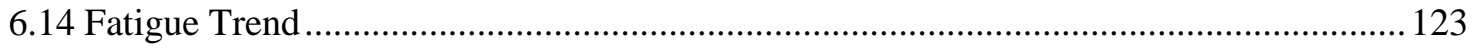

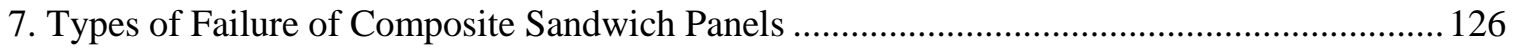

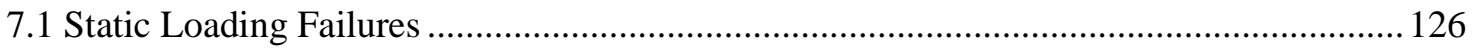

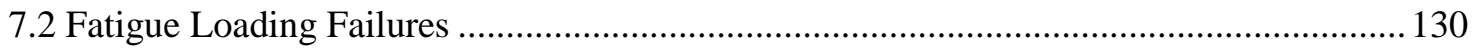

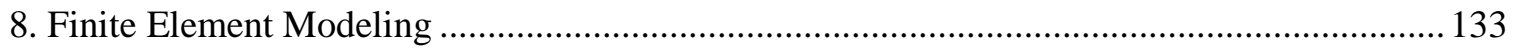

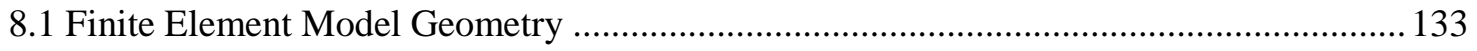

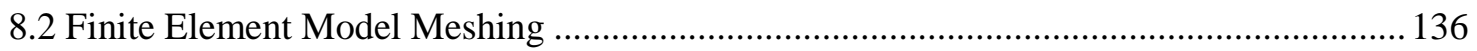

8.3 Finite Element Model Static Loading/Displacement ...................................................... 141

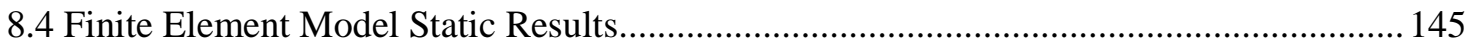

9. Comparison between Theoretical, Experimental and FEM .................................................. 168

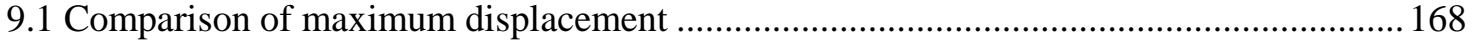

9.2 Comparison of load/extension curve and Elastic Modulus.............................................. 169

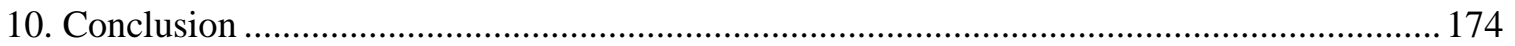

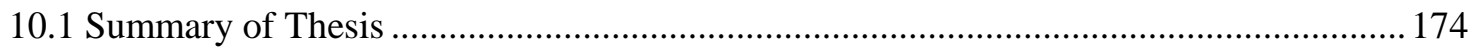

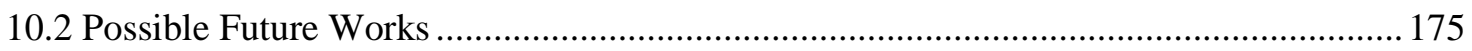

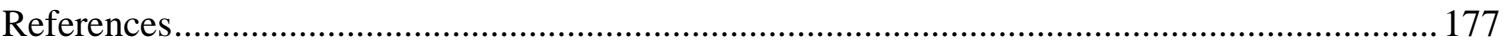




\section{List of Tables}

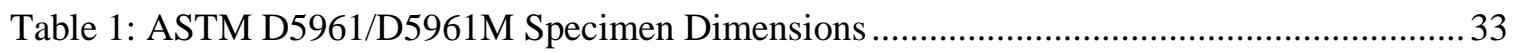

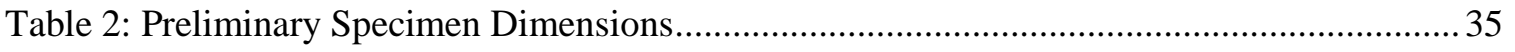

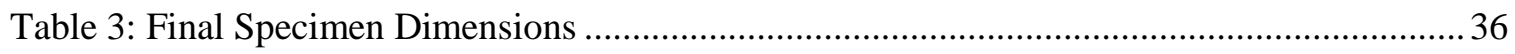

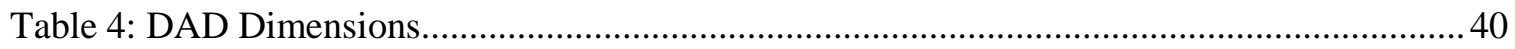

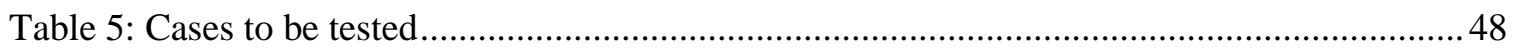

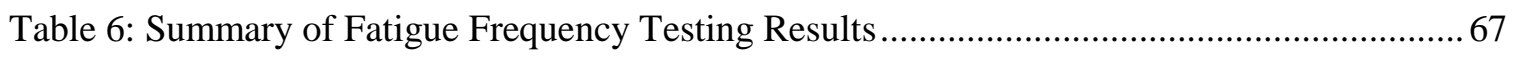

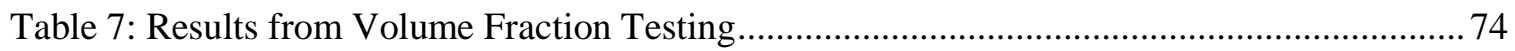

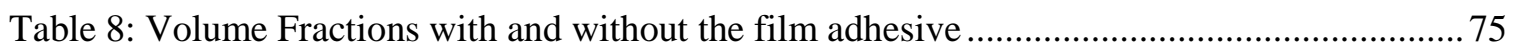

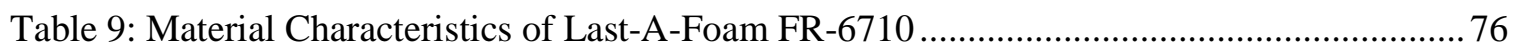

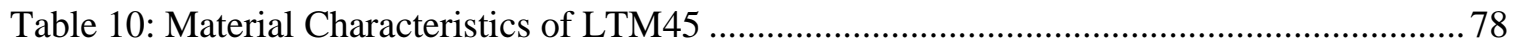

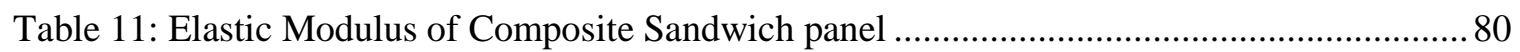

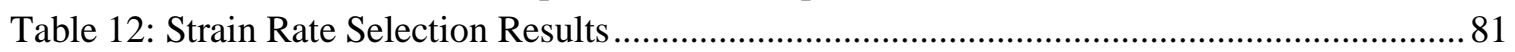

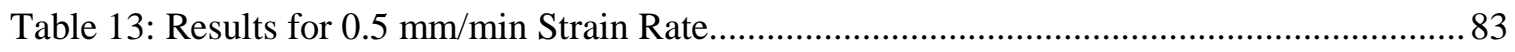

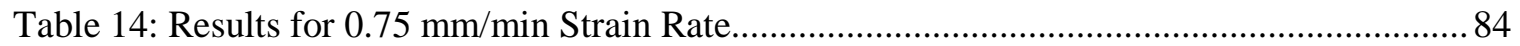

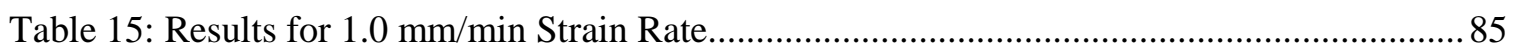

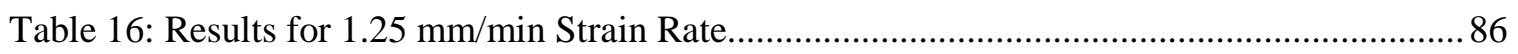

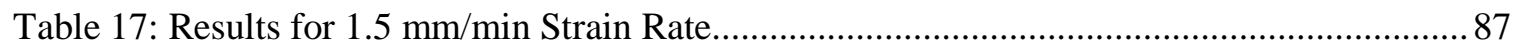

Table 18: Test Results for DAD Manufacturing Method 1 ............................................................ 91

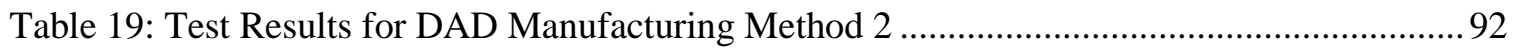

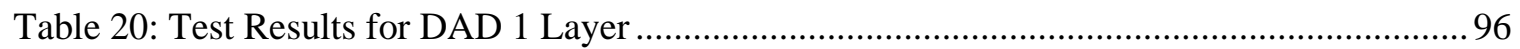

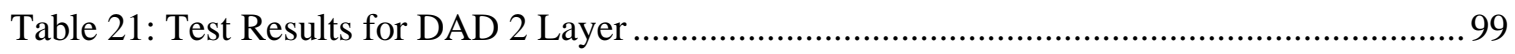

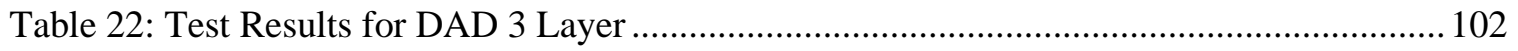

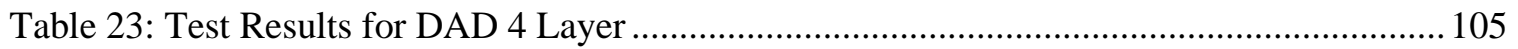

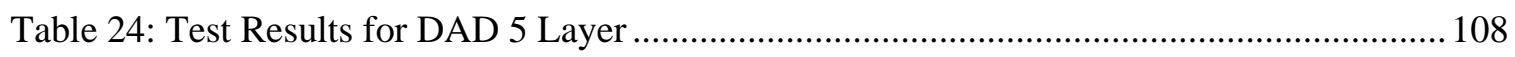

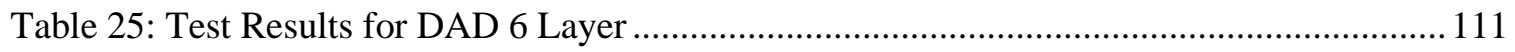

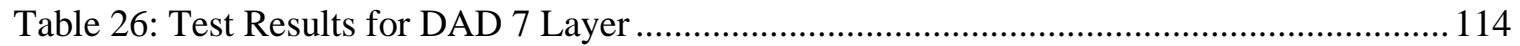

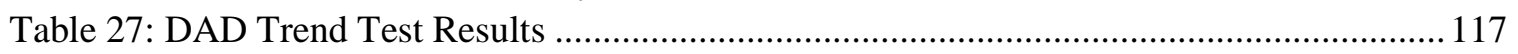

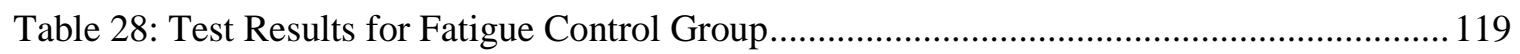

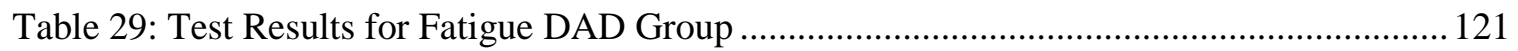

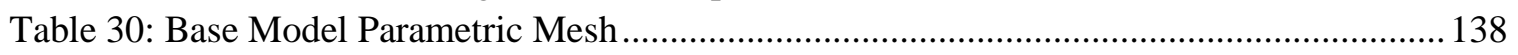

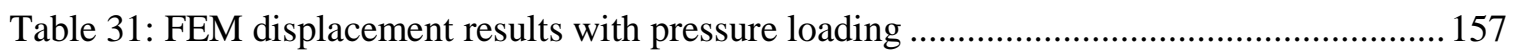

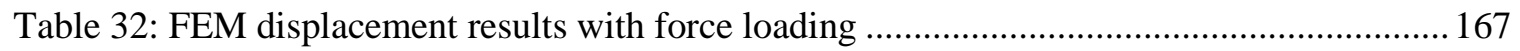

Table 33: Comparison of maximum displacement between Experimental and FEM Results..... 168

Table 34: Elastic Modulus Comparison for Control Group.....................................................171

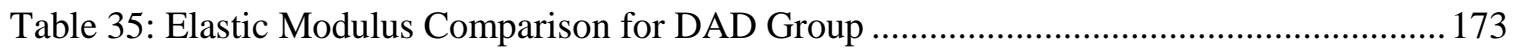




\section{List of Figures}

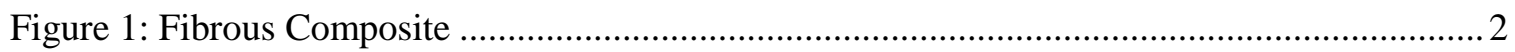

Figure 2: Laminated Composite (Courtesy of Picses EU) …...................................................... 3

Figure 3: Particle Composite (University of Illinois Engineering) ................................................ 4

Figure 4: Strength and Stiffness of Advanced Composite Materials ............................................. 5

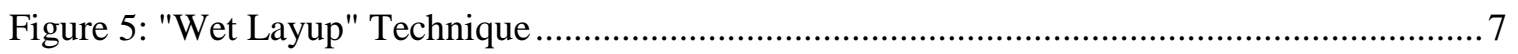

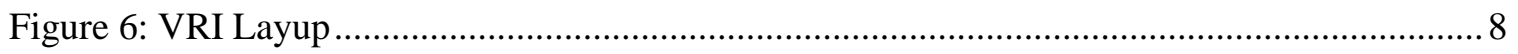

Figure 7: Pre-preg Layup (Courtesy of Composite World) ..................................................... 9

Figure 8: Example of Sandwich Composites (Courtesy of Engineer Materials Inc) ...................... 10

Figure 9: Example of Composite Sandwich Panels in Aircraft Structures ................................... 11

Figure 10: An FEA model of an aircraft fuselage. (Courtesy of McGettrick Engineering) .......... 13

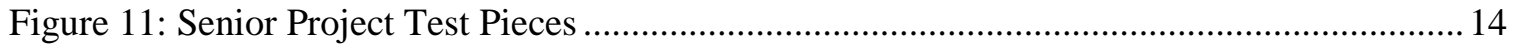

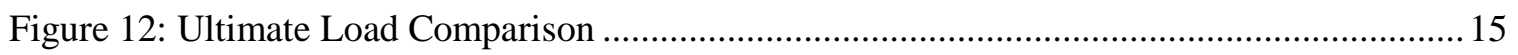

Figure 13: Examples of Failure Modes for Fastener/Joint Interaction ........................................16

Figure 14: Empirical Observation of Hole Placement on Composite Panel ...................................17

Figure 15: Shear Testing by Demelio et al ............................................................................ 18

Figure 16: Types of failure seen during testing by Demelio et al ............................................... 19

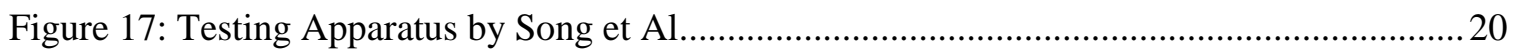

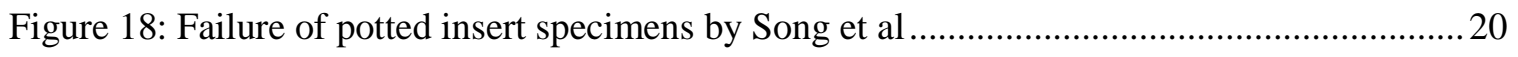

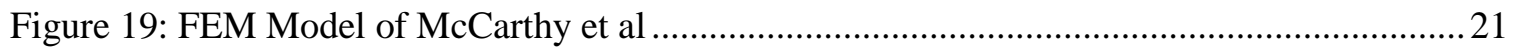

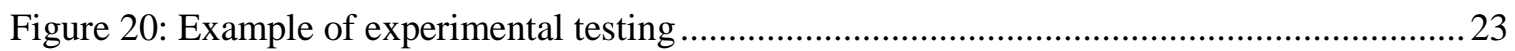

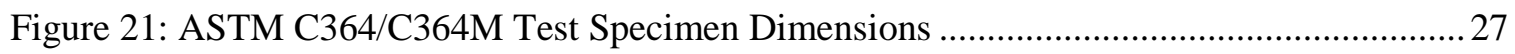

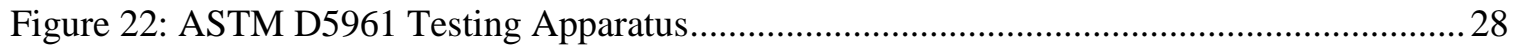

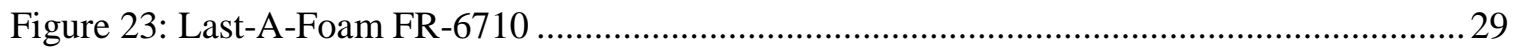

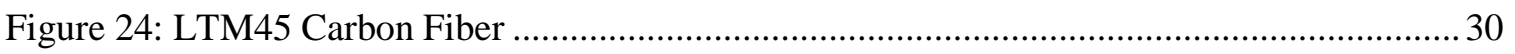

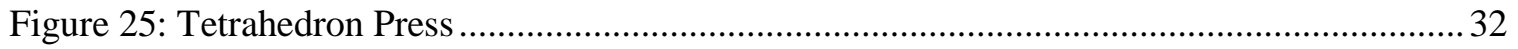

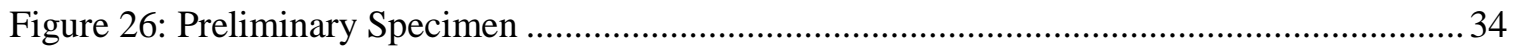

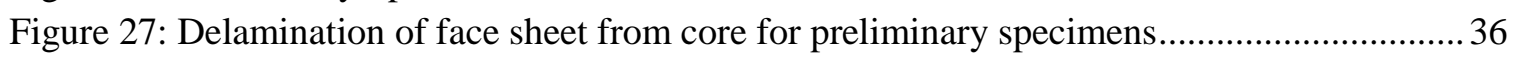

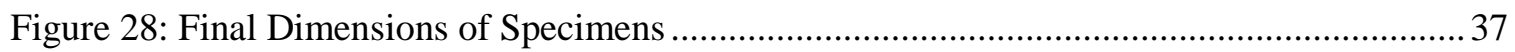

Figure 29: Circular Damage Arrestment Devices from Balatbat et al......................................38

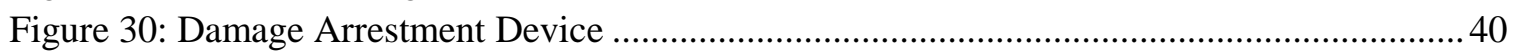

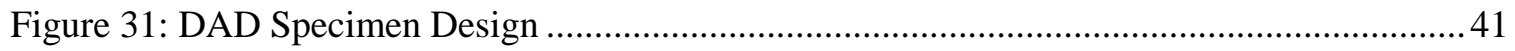

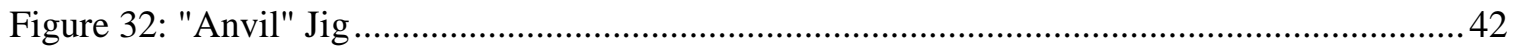

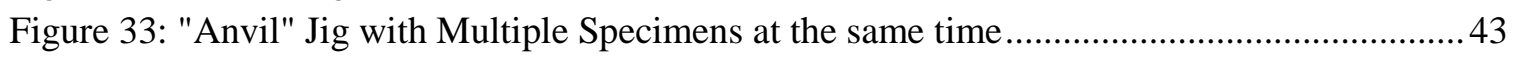

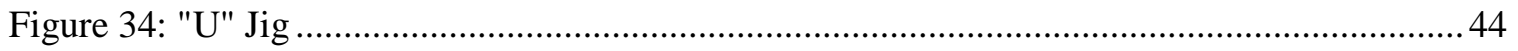

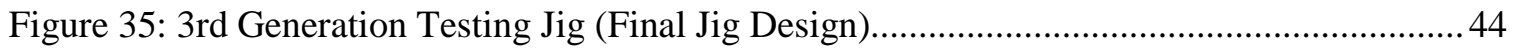

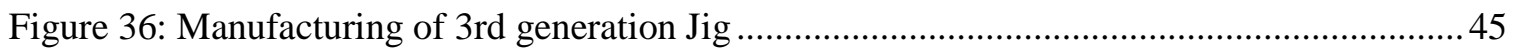

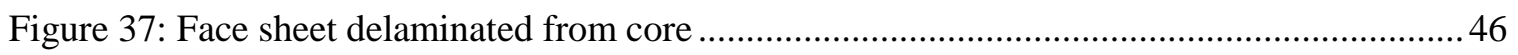

Figure 38: Bits used for hole drilling (Left: Conical Sanding Drill Bit/Right: Glass and Tile Drill

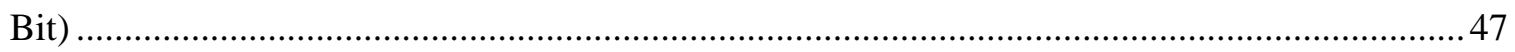

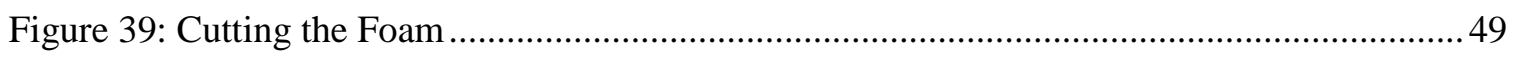

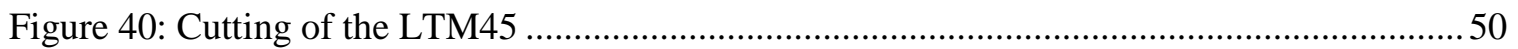

Figure 41: Schematic for Lay-up of Composite Sandwich Panels ............................................. 51 


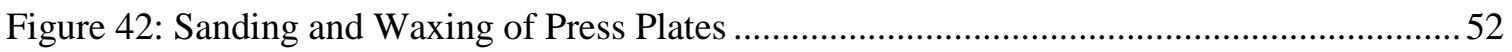

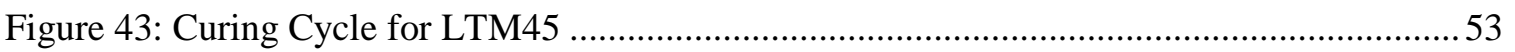

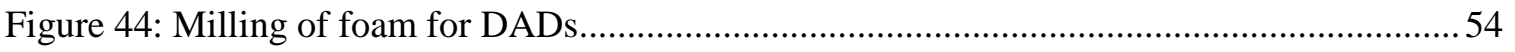

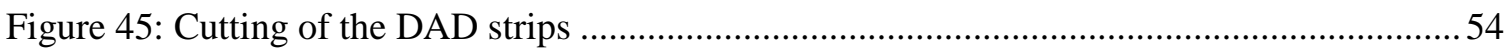

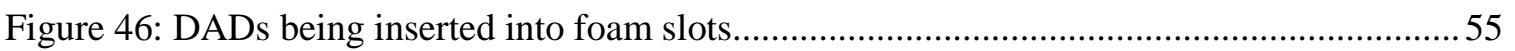

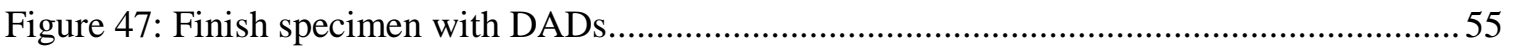

Figure 48: Cutting of Composite Sandwich Plates without DADs ............................................... 56

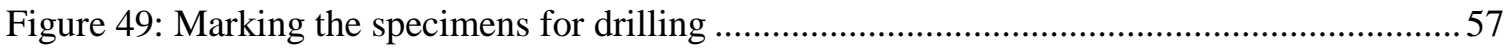

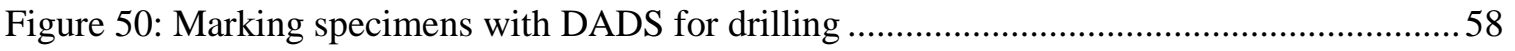

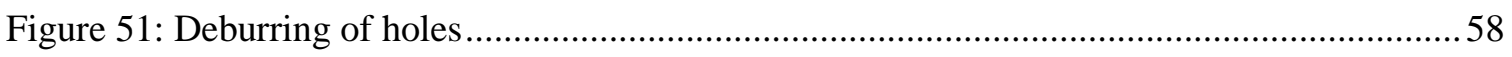

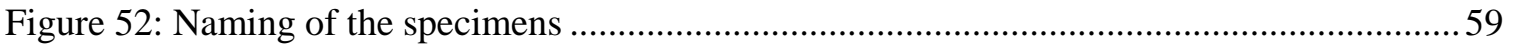

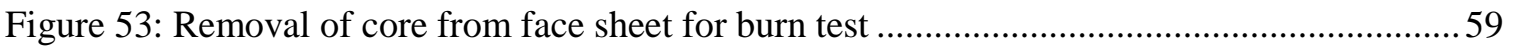

Figure 54: Measurements of specimens for volumetric fraction testing.......................................60

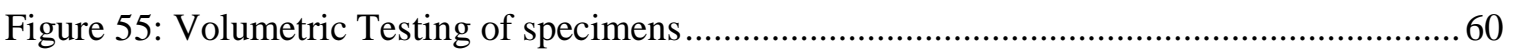

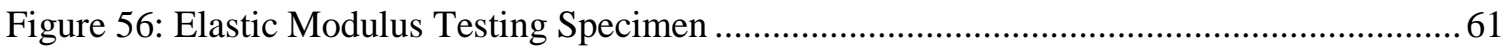

Figure 57: Manufacturing of Elastic Modulus Testing Specimens..............................................62

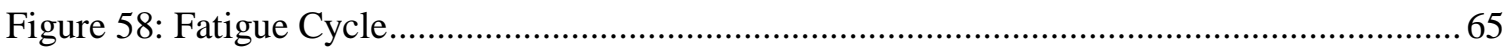

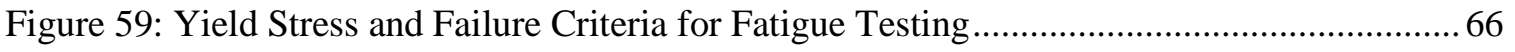

Figure 60: Example of recording measurements in Goggle Docs ............................................... 68

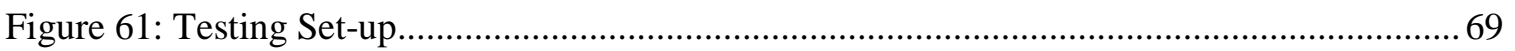

Figure 62: Example of laminate heights (Courtesy of Agarawal) ............................................... 73

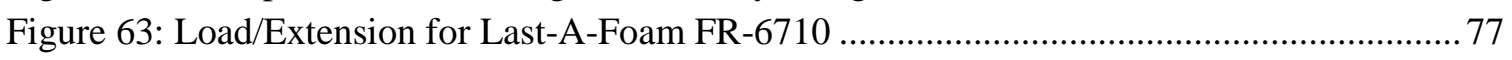

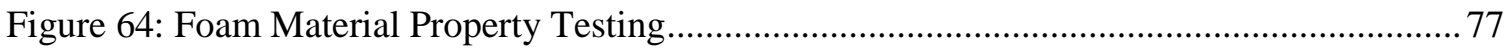

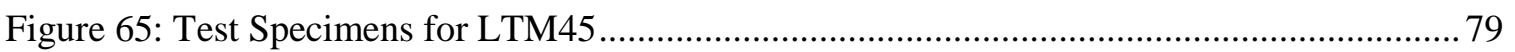

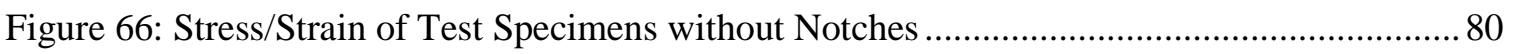

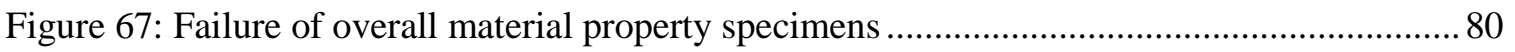

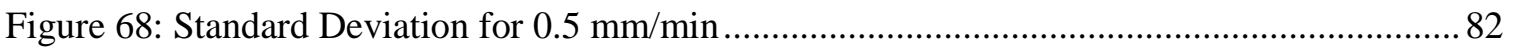

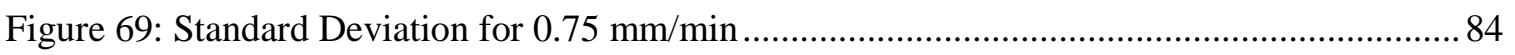

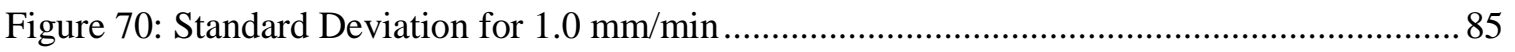

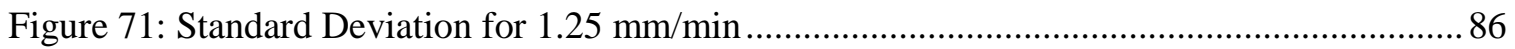

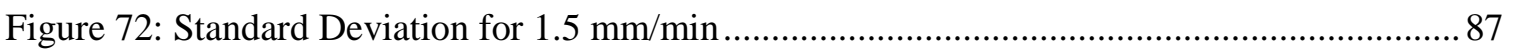

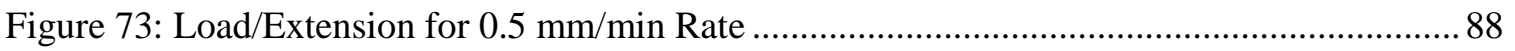

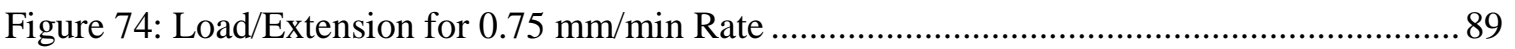

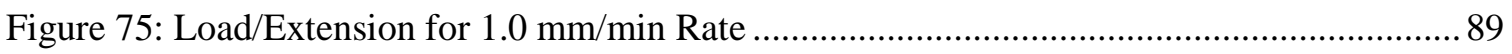

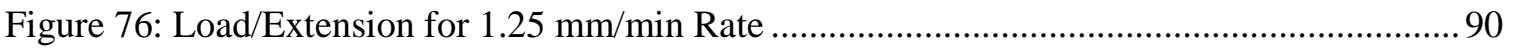

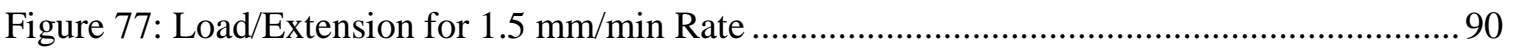

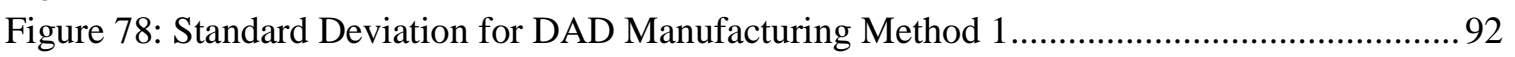

Figure 79: Standard Deviation for DAD Manufacturing Method 2............................................. 93

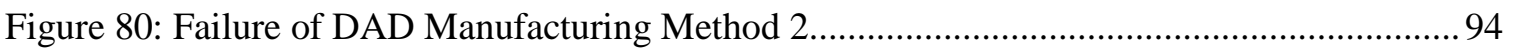

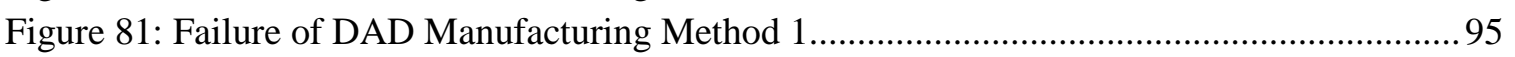

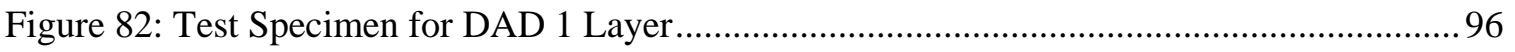

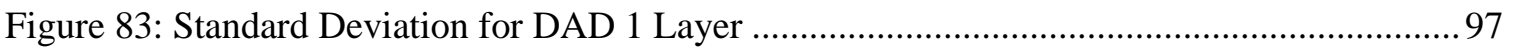

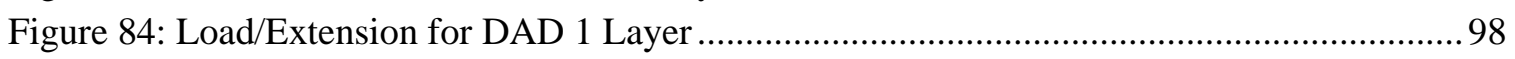

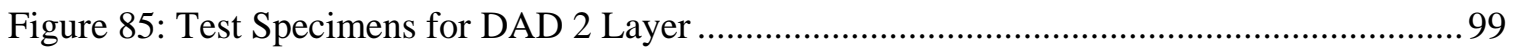




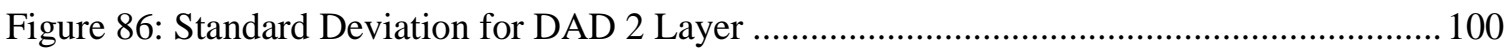

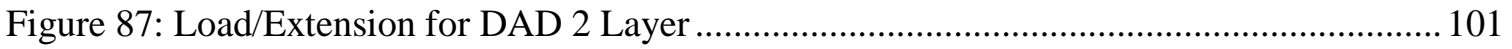

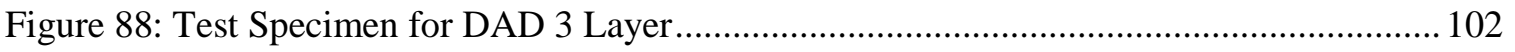

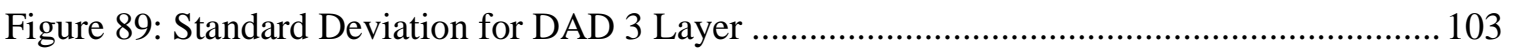

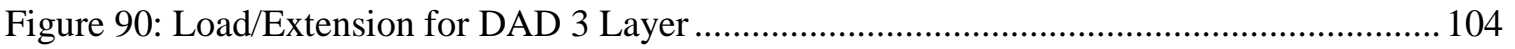

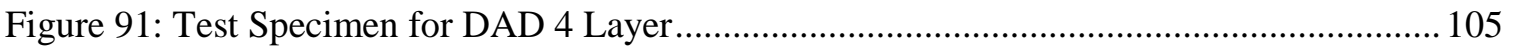

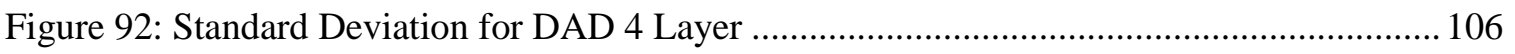

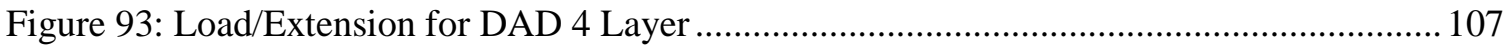

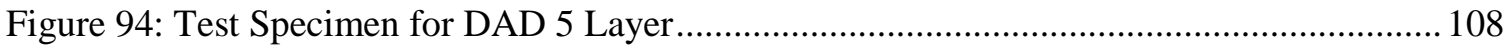

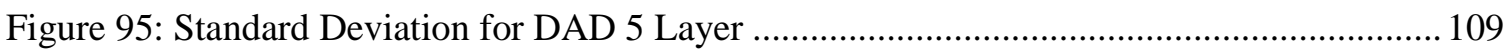

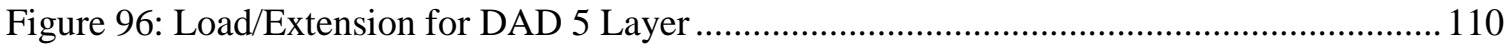

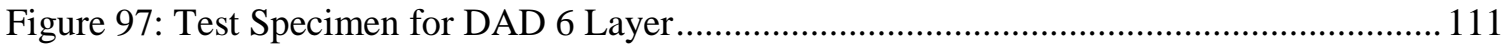

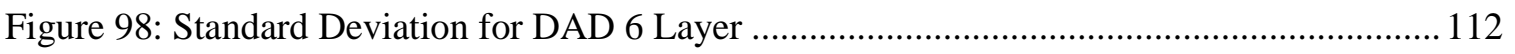

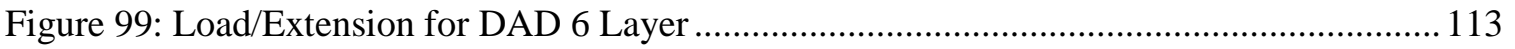

Figure 100: Test Specimens for DAD 7 Layer ....................................................................... 114

Figure 101: Standard Deviation for DAD 7 Layer ................................................................115

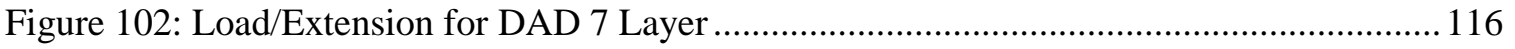

Figure 103: Ultimate Load DAD thickness trend ....................................................................118

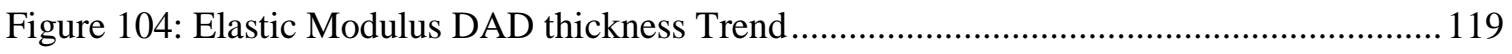

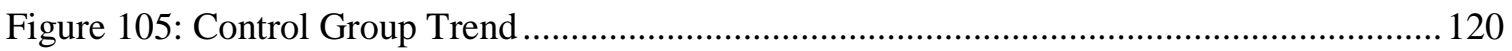

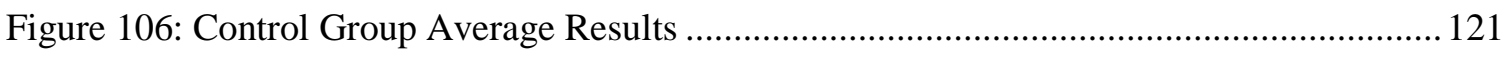

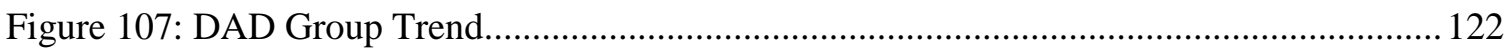

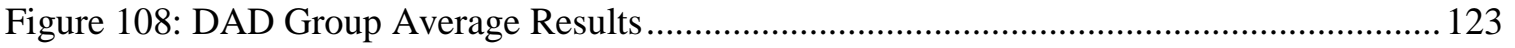

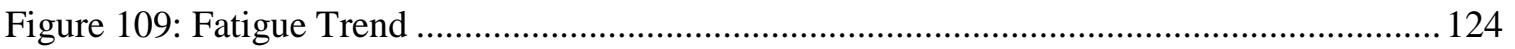

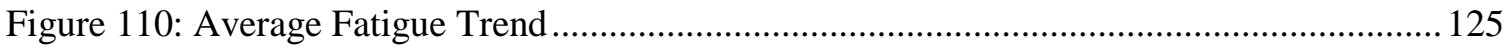

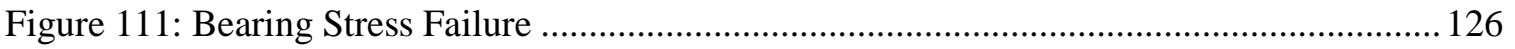

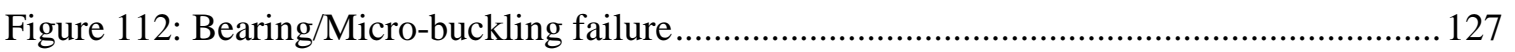

Figure 113: Bearing Stress on high thickness DADs............................................................ 128

Figure 114: Complete failure of composite sandwich panel....................................................... 129

Figure 115: Complete failure with buckling of composite sandwich panel................................ 130

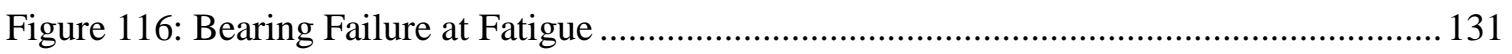

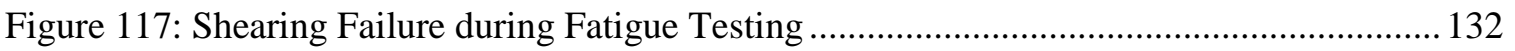

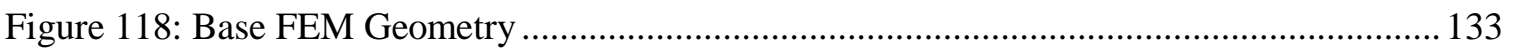

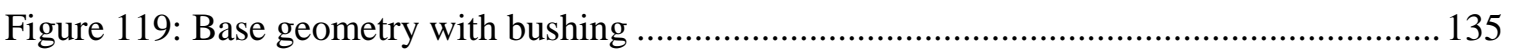

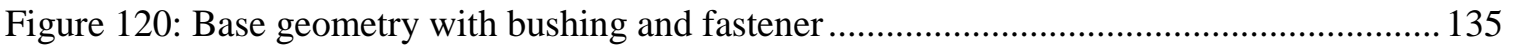

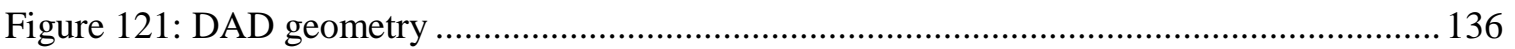

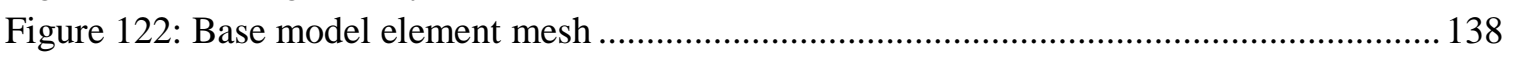

Figure 123: Base model with bushing element mesh .................................................................. 139

Figure 124: Base model with bushing and fastener element mesh ..........................................140

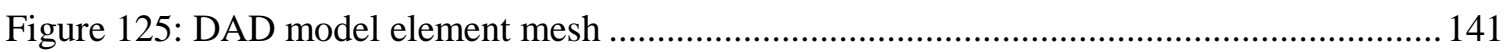

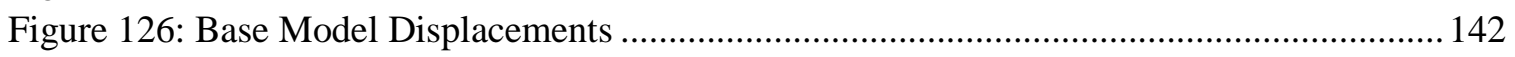

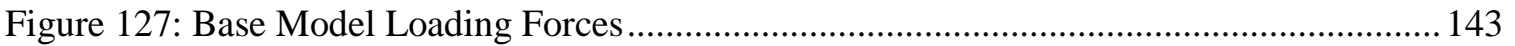

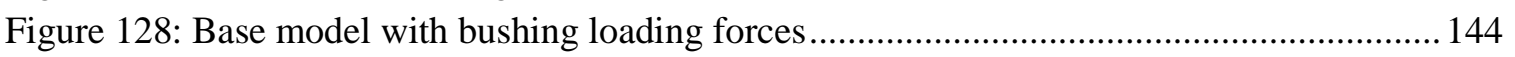

Figure 129: Base model with bushing and fastener loading forces .......................................... 144 
Figure 130: Force Loading on FEM Base Model 145

Figure 131: Displacement response of FEM base model with pressure loading ......................... 146

Figure 132: Stress response of FEM base model with pressure loading.....................................147

Figure 133: Displacement response of FEM base model with bushing and pressure loading.....148

Figure 134: Stress response of FEM base model with bushing and pressure loading ................. 149

Figure 135: Displacement response of FEM base model with bushing, fastener and pressure

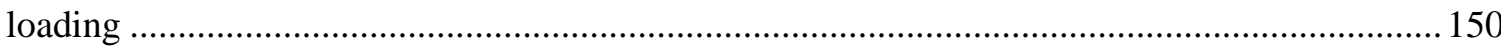

Figure 136: Stress response of FEM base model with bushing, fastener and pressure loading... 151

Figure 137: Displacement response of FEM DAD base model and pressure loading................. 152

Figure 138: Stress response of FEM DAD base model and pressure loading ............................. 153

Figure 139: Displacement response of FEM DAD base model with bushing and pressure loading

Figure 140: Stress response of FEM DAD base model with bushing and pressure loading........ 155

Figure 141: Displacement response of FEM DAD base model with bushing, fastener and pressure

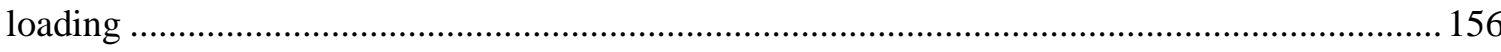

Figure 142: Stress response of FEM DAD base model with bushing, fastener and pressure loading

Figure 143: Displacement response of FEM base model with force loading ............................. 158

Figure 144: Stress response of base model with force loading .................................................. 159

Figure 145: Displacement response of FEM base model with bushing and force loading........... 160

Figure 146: Stress response of FEM base model with bushing and force loading ...................... 160

Figure 147: Displacement response of FEM base model with bushing, fastener and force loading

Figure 148: Stress response of FEM base model with bushing, fastener and force loading........ 162

Figure 149: Displacement response of FEM DAD base model with force loading...................... 163

Figure 150: Stress response of FEM DAD base model with force loading .................................163

Figure 151: Displacement response of FEM DAD base model with bushing and force loading 164

Figure 152: Stress response of FEM DAD base model with bushing and force loading............. 165

Figure 153: Displacement response of FEM DAD base model with bushing, fastener and force

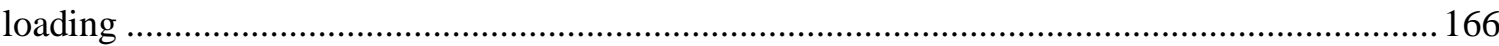

Figure 154: Stress response of FEM DAD base model with bushing, fastener and force loading

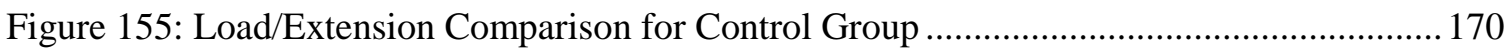

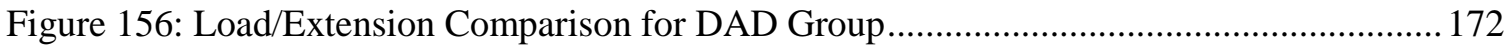




\section{List of Equations}

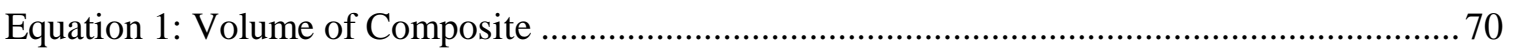

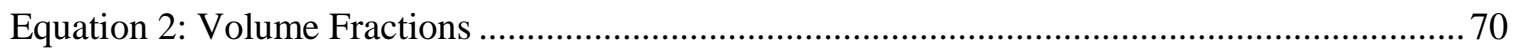

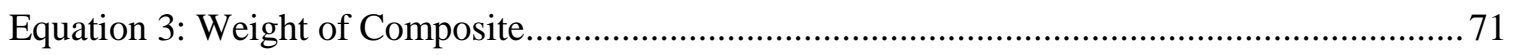

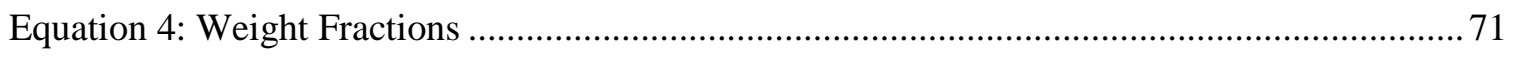

Equation 5: Generalized Equation of the Volume and Weight Fraction Relation......................... 71

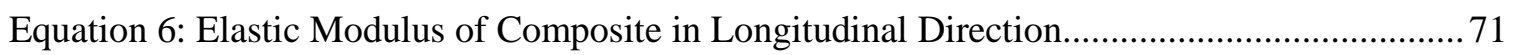

Equation 7: Elastic Modulus of Composite in Transverse Direction........................................... 71

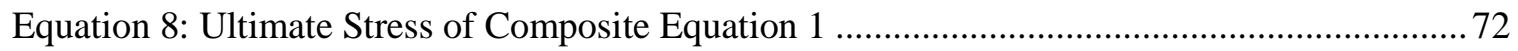

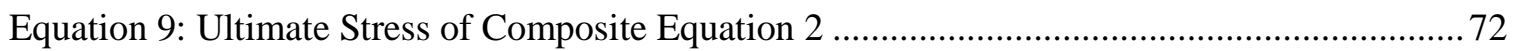

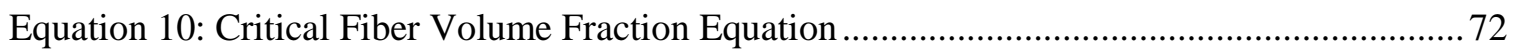

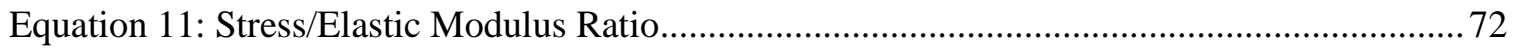

Equation 12: Generalized Form of the Extensional Stiffness Matrix ......................................... 73

Equation 13: Alternate Extensional Stiffness Matrix ............................................................... 73

Equation 14: General Constitutive Equation for forces with a symmetric plate............................ 73 


\section{List of Symbols}

\begin{tabular}{|c|c|c|}
\hline A & Amplitude & $(-)$ \\
\hline A & Extensional stiffness matrix & $(-)$ \\
\hline $\mathrm{E}$ & Elastic Modulus & $\left(\mathrm{lbs} / \mathrm{in}^{2}\right)$ \\
\hline $\mathrm{f}$ & Frequency & $(\mathrm{Hz})$ \\
\hline h & height & (in) \\
\hline K & stress concentration factor & $(-)$ \\
\hline $\mathrm{k}$ & Stiffness & (lbs/in) \\
\hline $\mathrm{N}$ & Force/load & (lbs) \\
\hline $\mathrm{P}$ & Force/load & (lbs) \\
\hline Q & Reduced stiffness matrix & $(-)$ \\
\hline $\mathrm{R}^{2}$ & Coefficient of determination & $(-)$ \\
\hline $\mathrm{t}$ & Thickness & (in) \\
\hline $\mathrm{V}$ & Volumetric fraction & $(-)$ \\
\hline $\mathrm{V}$ & Volume & $\left(\mathrm{in}^{3}\right.$ or $\left.\mathrm{cm}^{3}\right)$ \\
\hline $\mathrm{W}$ & Weight fraction & $(-)$ \\
\hline $\mathrm{W}$ & Weight & (oz or g) \\
\hline $\mathrm{X}$ & Coordinate Direction & $(-)$ \\
\hline $\mathrm{Y}$ & Coordinate Direction & $(-)$ \\
\hline $\mathrm{Z}$ & Coordinate Direction & $(-)$ \\
\hline$\varepsilon$ & Strain & $(-)$ \\
\hline$\rho$ & Density & $\left(\mathrm{g} / \mathrm{cm}^{3}\right)$ \\
\hline$\sigma$ & Stress & $\left(\mathrm{lbs} / \mathrm{in}^{2}\right)$ \\
\hline$v$ & Poisson’s Ratio & $(-)$ \\
\hline \multicolumn{3}{|c|}{ Subscript } \\
\hline $\mathrm{C}$ & Composite & \\
\hline cr & Critical & \\
\hline crit & Critical & \\
\hline $\mathrm{cu}$ & Longitudinal strength of composite & \\
\hline$e_{f}$ & Matrix stress at fiber fracture strain & \\
\hline$f$ & Fiber & \\
\hline fu & Ultimate strength of fiber & \\
\hline $\mathrm{i}$ & Matrix row & \\
\hline j & Matrix column & \\
\hline $\mathrm{k}$ & Layer of laminate from center & \\
\hline $\mathrm{m}$ & Matrix & \\
\hline $\mathrm{sb}$ & Shear Stress & \\
\hline tb & Tensile Stress & \\
\hline $\mathrm{y}$ & Yield & \\
\hline ult & Ultimate & \\
\hline 1 & Longitudinal direction & \\
\hline 2 & Axial direction & \\
\hline
\end{tabular}




\section{Introduction}

A brief discussion on all things composites was talked about in this chapter to familiarize the reader on the subject matter. Composite are defined by the types of composite materials, on how they are manufactured, and their advantages and disadvantages. The application and research of composites such as composite sandwich panels in industry are also discussed. Lastly, the purpose of the research pertaining to composite sandwich panels was discussed at the end of the chapter.

\subsection{Overview of Composites}

\subsubsection{Introduction of Composites}

Composite materials have been used since the beginning of human history. The earliest recorded use of composites was in ancient Egypt where they created bricks from mud and straw to build their buildings. They also achieved higher strength and better resistance to thermal expansions to their bricks by adding plywood to it. Swords and armor were created with different layers of materials to improve strength during Medieval Europe.

A composite was defined as a material that contains two or more constituents that are combined on a macroscopic scale to form a new material. Composites exhibit the best qualities of their constituents and often some qualities that neither possesses. Some of the properties improved by composite materials include strength, stiffness, corrosion resistance, wear resistance, attractiveness, weight, fatigue life, temperature-dependent behavior, thermal insulation, thermal conductivity, and acoustical insulation. Creating a composite material does not improve all of the properties. The designer can choose which properties to improve by choosing the right pieces to combine. Laminated composites are used to combine the best aspects of the constituent layer in order to achieve a more useful material. 


\subsubsection{Types of Composites}

There are three types of composites: fibrous, laminated and particulate composites. All three types are made out of two constituents, the actual composite material and the binding agent or the matrix. The composite material can be a type of metal or a fiber. The binding agent or the matrix can be another type of metal or some type of adhesive material. The two constituent materials are combined with another to create a hybrid material that was much stronger and stiffer.

Fibrous composites consist of fibers in a matrix; this can be seen in Figure 1. Fibers in fibrous composites are much stiffer and stronger than the same material in its bulk form. The geometry of the fiber was crucial to its strength and must be considered in structural applications. The difference between the strength of a fiber and the strength of its bulk material was due to the crystal alignment along the fiber axis. There are also fewer defects in fibers than its respective bulk material.

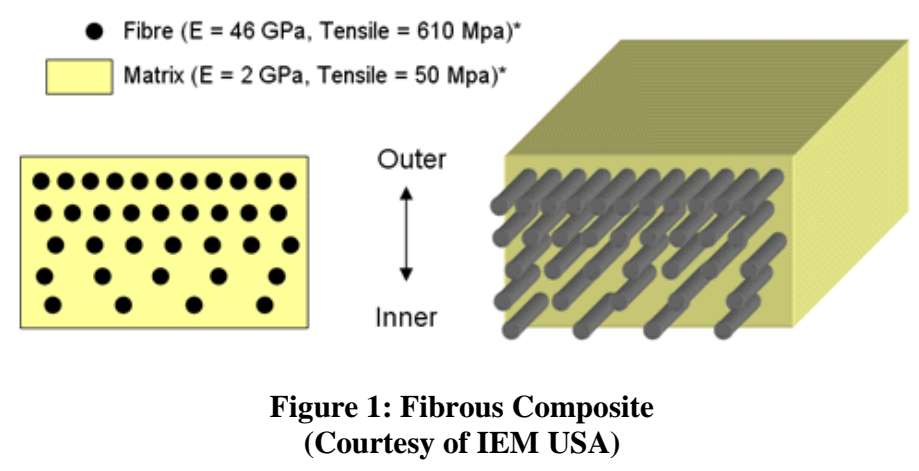

The purpose of the matrix was to support, protect, and transfer the stresses along the fibers. The matrix has a lower density, stiffness, and strength than the fibers. The combination of the fibers and the matrix can create a very strong and stiff material while still maintaining a low density.

A laminated composite can consist of layers of at least two different materials bound to one another; this can be seen in Figure 2. Some classes of laminated composites are bimetals, clad metals, laminated glass, plastic based laminates, and laminated fibrous composites. Bimetals are 
laminates of two different metals with significantly different coefficients of thermal expansion. Clad metals are the cladding or sheathing of one metal with another, which was done to obtain the best properties of both metals. An example was high-strength aluminum alloy covered with a corrosion-resistant aluminum alloy. This was a composite material with unique and attractive advantages over regular aluminum which was very corrosive. Plastic based laminates are materials that are saturated with various plastics to improve its material property. Laminated fibrous composites are a hybrid of composites involving both fibrous composites and lamination techniques; this was also known as laminated fiber-reinforced composites. Layers of fiberreinforced material are built with the fiber directions of each layer typically oriented in different directions to give different strengths and stiffness in various directions.

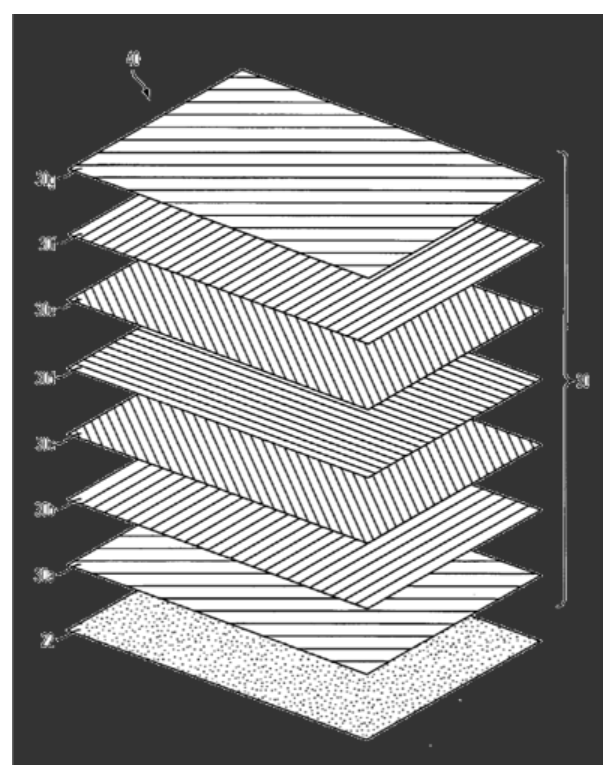

Figure 2: Laminated Composite (Courtesy of Picses EU)

Particulate composites are composed of particles in a matrix; this can be seen in Figure 3. Particles are non-fibrous and generally have no long dimension. The particles and the matrix can be either metallic or nonmetallic. The choice of a particular combination depends upon the desired properties of the final material. The most common example for a particulate composite was concrete. Concrete consist of small sand and rock particles bonded together by a mixture of 
cement and water that chemically reacts and hardens. The strength of the concrete was comparable to rock.
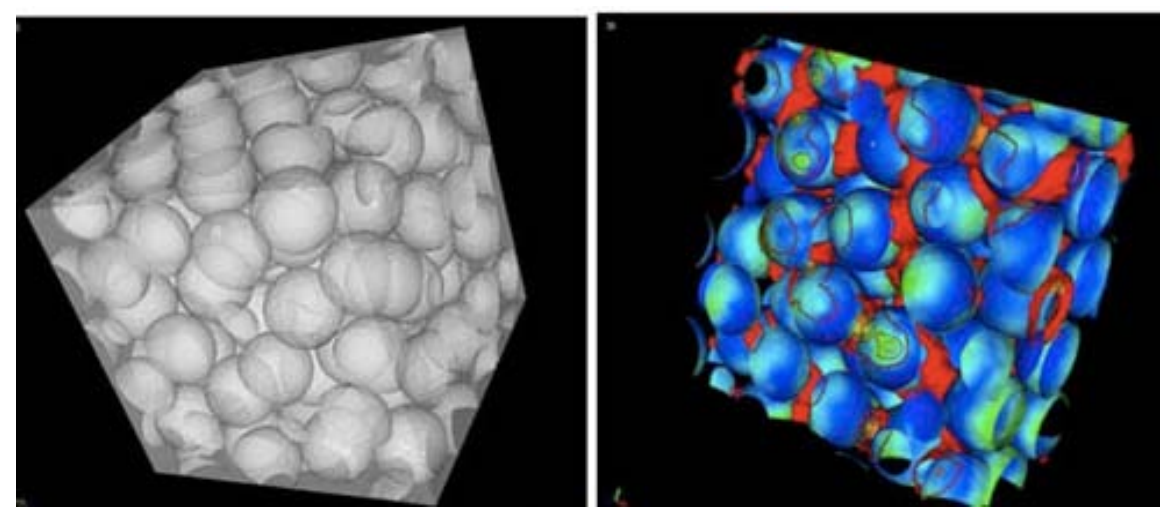

Figure 3: Particle Composite (University of Illinois Engineering)

\subsubsection{Advantages and Disadvantages of Composites}

Composites have two major advantages: improved strength and stiffness, especially when compared with other materials on a unit weight basis. The best way of showing the effectiveness of strength or stiffness of a material was the weight per unit volume, as seen in Figure 4. It shows a comparison of the strength and stiffness of several types of advanced composite materials and contemporary metals. The most desired material in the figure was located in the upper right hand corner of the figure, which represents a material with high strength and stiffness. It shows that composite materials are stronger and stiffer than common metals such as aluminum, steel, and titanium, while being lighter than those metals. 


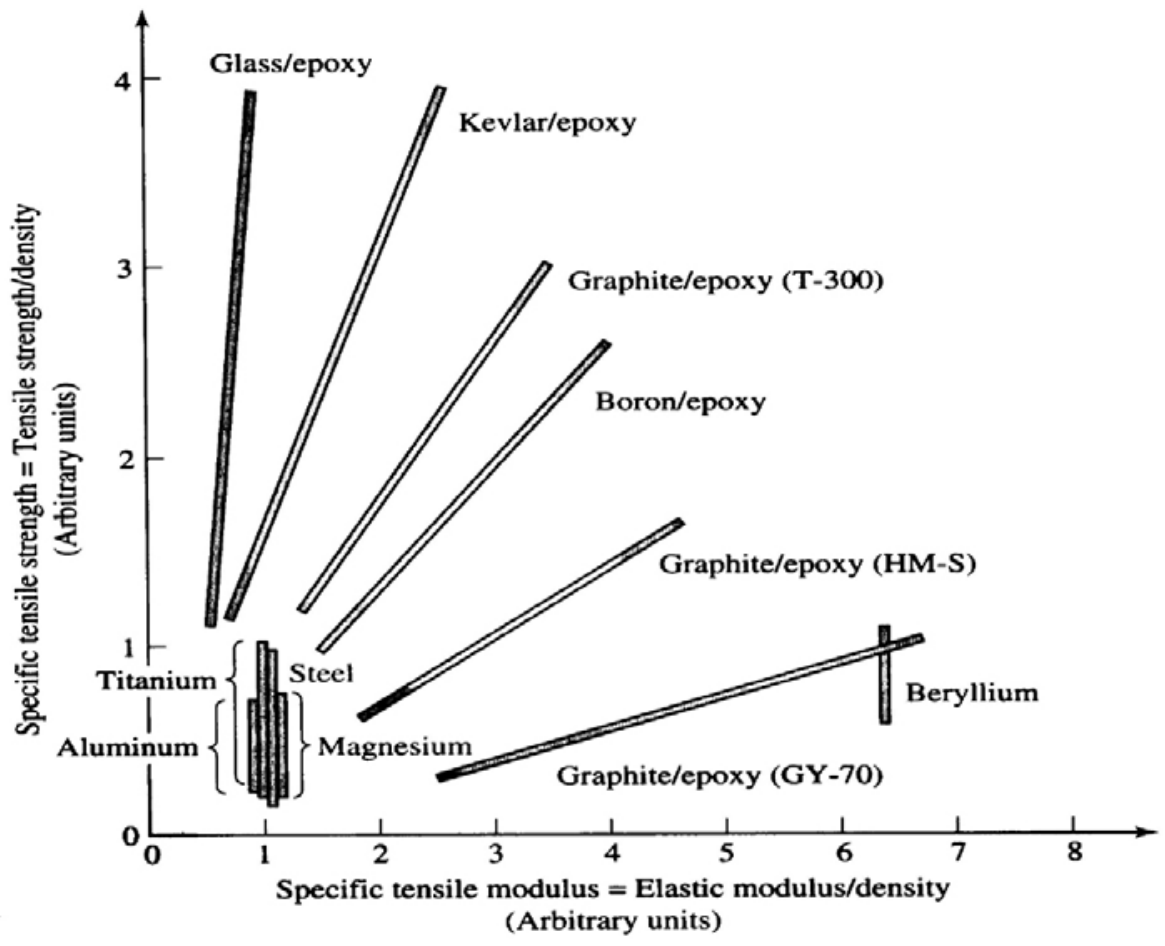

Figure 4: Strength and Stiffness of Advanced Composite Materialsvii ${ }^{\text {ii }}$

Properties of composites are strongly influenced by the properties of the materials they're composed of, the material distribution, and the interaction between the materials. Properties of the composite are mainly dependent on the geometry of the re-enforcement of the composite material such as shape, size, and orientation. Size and distribution control of the material determines the interaction between the fiber and the matrix.

Materials are stronger and stiffer in the fibrous form as in its bulk material form. A high fiber aspect ratio permits very effective transfer of load via matrix materials to the fibers, making a very effective reinforcement material. There are several types of fibers used in composites including glass, carbon, graphite, aramid, boron, and ceramic. Glass fibers are the most common fiber used for composite materials because of their low cost and high strength. Carbon and graphite fibers are mostly high-strength, high-modulus fibers that are used for high-performance composites. Aramid fibers, or Kevlar, are a type of fiber that can change its material property depending on how the material was created. Kevlar fibers have higher tensile strength and 
modulus, but have a lower fiber elongation than glass or carbon fibers. Boron fibers have a higher tensile strength than carbon fibers, but the fibers are more expensive to produce than other fibers. Ceramic fibers were created because of the need for high temperature fibers. Ceramic fibers combine high strength and elastic modulus with higher temperature capability than other types of fiber materials.

The matrix of a composite material binds the fibers together, transfers loads between them, and protects them against environmental degradation and damage due to handling. The matrix has a great influence on the composite material because it defines several material properties such as shear, compression, transverse modulus, and strength properties. It also limits a materials temperature property by defining the maximum operational temperature the composite material can work in. Polymers or plastics are the most widely used matrix material for fiber composites because of their low cost, ease of production, chemical resistance, and low specific gravity. One of the main disadvantages of polymers was their low strength, low modulus, and low operating temperature. The most common polymers used for a composite matrix are polyester resin and epoxy resins. Metals can also be used as a matrix material for composites. Metals have a high strength, high modulus, high toughness, impact resistance, and its insensitivity to temperature changes over polymers. The main problems associated with use of metals as a matrix was their high density, high processing temperatures, reactivity with fibers, and corrosiveness.

\subsubsection{Types of Manufacturing}

There are three different common types of layup processes used for creating composite pieces. The simplest of these layup processes was the "wet layup” technique; this can be seen in Figure 5. This technique involves the least amount of materials and preparation time out of the three methods, but comes at the cost of the quality of the part. A wet layup was one in which the resin was pushed through the fibers by hand, and then placed inside a vacuum sealed bag and allowed to cure. Major advantages for this layup include low cost relative to the other layup 
processes, no special materials or additional equipment was required, the part was normally capable of curing at room temperatures, and the method was basic so that almost anyone can use it. Some disadvantages associated with this method include restricted work time (based on the resin), inconsistencies in resin to fiber ratio or the part, as well as destroying fiber integrity while working resin through the fibers.

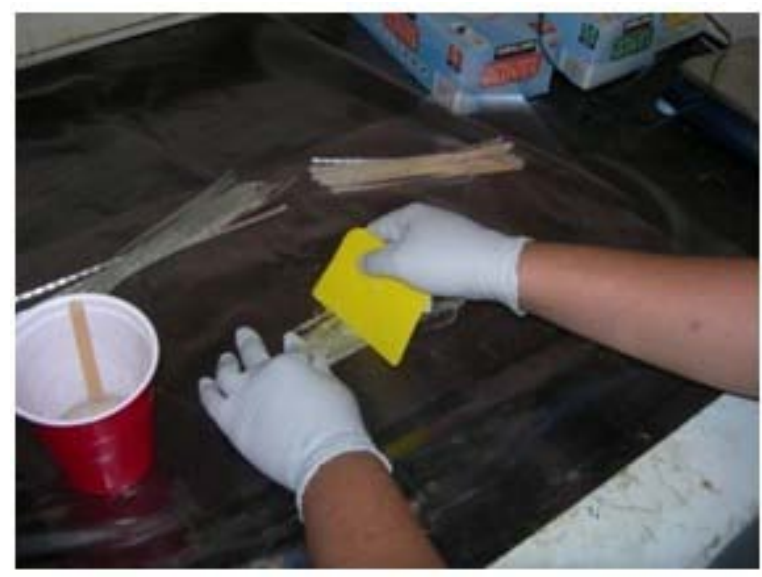

Figure 5: "Wet Layup" Technique

Another method typically used was called a resin infusion process or a vacuum resin infusion (VRI) process; this can be seen in Figure 6. This method has the most variation from person to person since each individual typically uses a slightly different setup and process. However, the main idea behind this method was that a vacuum was used to pull resin through the part. The advantage to this was a more consistent resin to fiber ratio throughout the whole part similar to that of the pre-preg but at a greatly reduced cost. However it does not require a complex cure cycle nor do the materials need to be stored to preserve a "shelf life" like the prepreg. It was also a preferred method for repeated experiments over a wet layup since the parts are reproducible and provide more consistent results. However this process does require more knowledge of the materials being used. Work time was dependent on the viscosity of the resin as well as the strength of the pump. The size of the part must also be taken into account since the resin may start to cure before reaching the other side of a larger part. VRI requires more materials 
and preparation time than either of the other two methods. A flow media was needed in order to allow resin to flow across the part, a peel-ply was placed under this flow media in order to remove it from the part, and additional tubing was required for the resin to be sucked through. This makes this method more costly then the wet layup, but allows the fabricator to avoid tampering with the fibers and causing misalignments in the part.

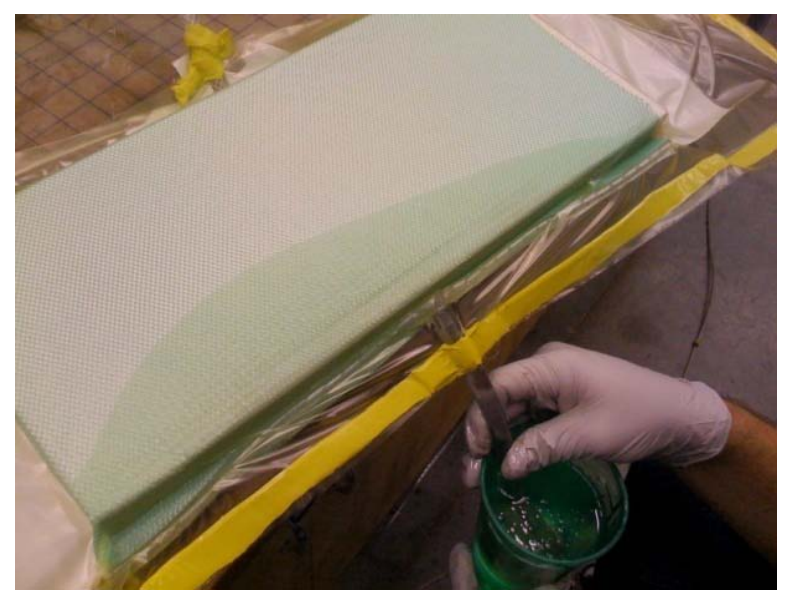

Figure 6: VRI Layup

The final method used in industry involves the use of pre-impregnated fibers also known as "pre-preg"; this can be seen in Figure 7. This method was ideal for almost any application in which money was not an issue. The extreme advantage with the pre-preg was that it already has the ideal resin to fiber ratio giving the part the maximum strength to weight ratio. The method gives the best consistency and higher quality in fabrication of the parts due to the premade resin to fiber ratio. Another advantage of pre-preg was the work time which was significantly longer then the wet layup technique, which starts to cure when the hardener and the epoxy are mixed. However, the disadvantage of this was that the manufacturer specifies the work time for pre-preg, and after the specified "shelf life" has expired, the material was no longer usable. Due to the “shelf life”, a freezer was needed to store the pre-preg in order to prevent it from losing "shelf life” when it was not being used. Pre-preg requires a heating cycle in order for the part to cure; 
meaning some type of machine has to be used to create the heating cycle. This includes autoclaves and heat presses to cure the pre-preg composite.

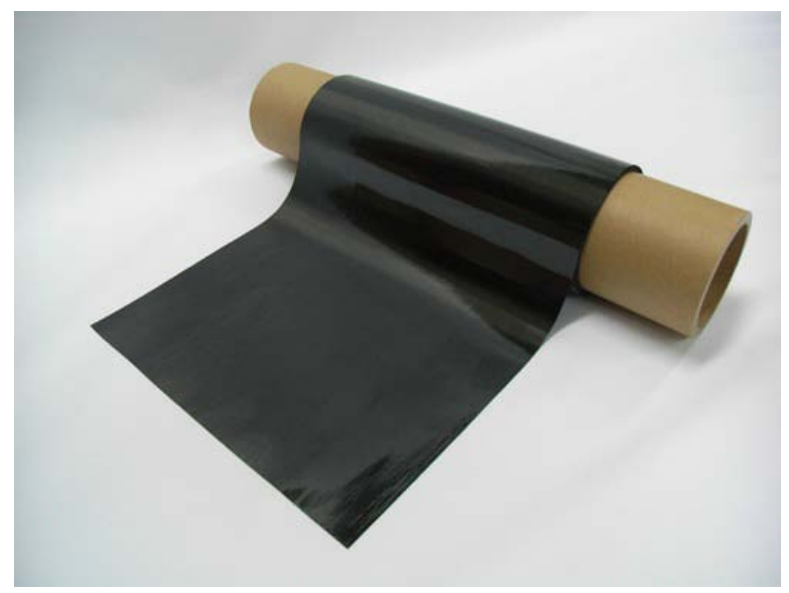

Figure 7: Pre-preg Layup (Courtesy of Composite World)

\subsubsection{Composite Structures}

Composite structures are any structures that contain a fibrous or laminated composite attached to it. The most common type of composite structure that will be used was sandwich composite panels. They widely used in several industries such as the marine, aerospace, and automotive, this can be seen in Figure 8. For application in the aerospace industry, fuselage hulls are made of panels of a foam core sandwiched between layers of carbon fiber. There are major advantages of sandwich composite panels such as decrease in weight and an increase in structural strength. Some of the major drawbacks are low shear strength and delamination of the composite skin from the core. The sandwich composite panels are susceptible to in-plane shear resisted primarily by the core, core compression failure, indentation of face-sheet subjected to impact loading, global buckling and wrinkling instability, and delamination of the face sheet from the core. In a structure with a delaminated surface that was loaded in compression, the face sheet over the delaminated region may buckle allowing the delamination to propagate through the panel causing the structure to fail. The shortcomings of fiber-reinforced plastics are that they have low mechanical properties and are susceptible to failure by impact loading. Low velocity impact on a 
sandwich structure can induce damage to the facings, the core and the core-facing interface. According to Castings ${ }^{\mathrm{i}}$, there are five different failure modes: core crushing, delamination in the impacted face sheet, core cracking, matrix cracking and fiber breakage in the facings. Damage of a composite panel was dependent on the properties and interaction of the core material and the facing of the plates.

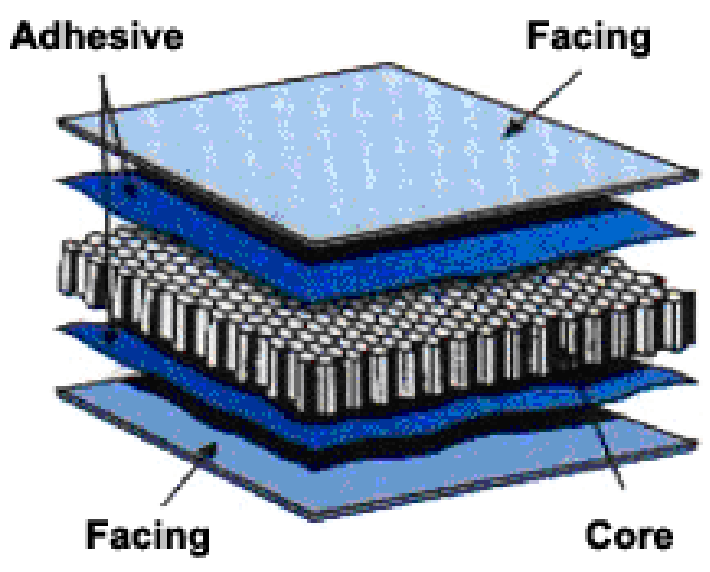

Figure 8: Example of Sandwich Composites (Courtesy of Engineer Materials Inc)

\subsubsection{Experimental Testing}

There are accepted testing standards where results could be compared with other researches by following a guideline on experimental testing. One of these standards was the American Standard for Testing and Materials (ASTM). It was an internationally recognized organization that develops and publishes consensus technical standards for materials, products, systems, and services. Most standards that pertain to composite testing are for the compression of composite sandwich structures and for testing of composite laminates with fasteners. The standard's give a full detailed account on how the dimensions of the test specimens, the testing apparatus, and how to analyze the results of the testing to be comparable to other results. Since there are no definite or accepted standards to test composite sandwich panels with a fastener, a variation or evolution of some of the standards was used for the experimental testing to require 
the desired or predicted results of the research; this will be further discussed in 2.1 Testing Standards.

\subsection{Application of Sandwich Composite Structures/Problems}

Composite sandwich structures are being used more in the aerospace industry. They are being used from the infrastructure of the aircraft such as the wings, the control surfaces and the fuselage of the aircraft; this can be seen in Figure 9. It shows a picture of a broken section of the rudder of an aircraft that was made out of a honeycomb composite sandwich structure with bolts attaching it to the rest of the vehicle. Composite sandwich structures are also used in the interior of airliners such as overhead storage bins, passenger cabin class dividers, galleys, lavatories, and insulation. They are mainly used on aircraft because they are light weight and stronger than aluminum, which was greatly used in old aircrafts.

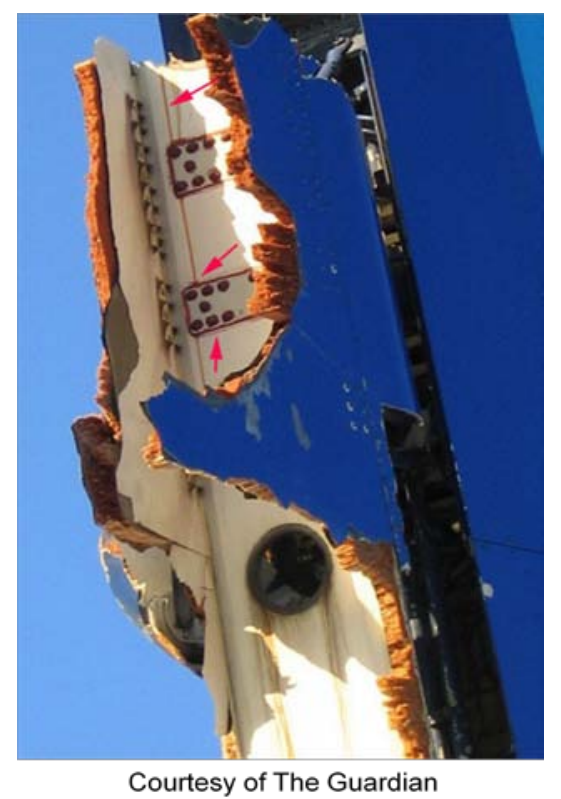

Figure 9: Example of Composite Sandwich Panels in Aircraft Structures

The structure of sandwich composite panels makes it ideal for both structural (load bearing) and non-structural (non load bearing) applications. The face sheets of a sandwich composite panel act similarly to the flanges on an I-beam because they resist the bending loads and increase the bending stiffness of the section by spreading the face sheets apart. This shape 
gives excellent stiffness and weight ratios to the structure. Also, good thermal and acoustic insulation properties exist in the core whose purpose was to bear shear loads to separate the face sheets and to carry loads from one face sheet to another.

Composite sandwich panels are weak at bearing concentrate loads, thus making it difficult to join panels. The conventional methods of joining composite structures to other composite or to metallic parts are adhesive bonding, mechanical fastening with rivets and joining by specially designed pieces. The core of the sandwich was normally light, soft, and weak making it susceptible to failure from mechanical joints. In order to install a mechanical joint, the core of the composite sandwich panel must be reinforced with some potting materials and inserts to sustain the fastener to the panel. Fasteners are then inserted to the insert.

Adding additional potting materials and a metallic insert to the composite sandwich panel increases the weight of the structure and decreases the overall stiffness of the panel. An alternative to this method was needed.

Damage arrestment devices (DADs) are some type of composite device that stops stress concentration from propagating. The purpose of the damage arrestment device was to prevent or prolonging failure in the specimen to propagate. This would increase the structural strength of the composite panel by prolonging the overall failure of the panel. These types of devices could be an alternative to potted inserts in a composite sandwich panel by having the same characteristics as potted inserts but are a lot lighter.

\subsection{Finite Element Modeling}

When an aircraft was conceptually designed for the aerospace industry, a structural analysis must be conducted to see if the aircraft was airworthy. To do this, the industry actually builds a model or replica of the aircraft to test if it was structurally sound. During a conceptual stage of design, engineers cannot build an actual model and test it. The method that was used to analyze the structural airworthiness of an aircraft was to use Finite Element Modeling (FEM) or 
Finite Element Analysis (FEA). The method was cheaper to analyze the structures of an aircraft; this can be seen in Figure 10. An engineer can obtain multiple structural results for an aircraft in different situations before a model can be built and tested.

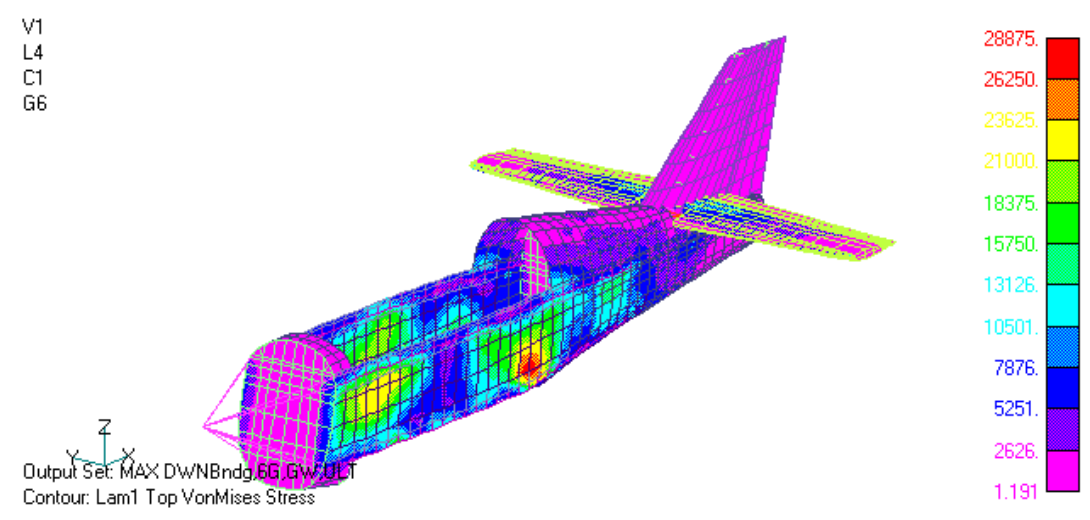

Figure 10: An FEA model of an aircraft fuselage. (Courtesy of McGettrick Engineering)

Finite element analysis was a numerical method for finding the numerical solution to PDEs that solve the stresses, strains, and displacement of an object. The purpose of FEA was to solve the stress/strain equations in a small section of the geometry and combine these results with the rest of the geometry to obtain the stress analysis of the object. It consists of a system of nodes that makes a grid of elements, which was called a mesh. Each element contains the material and structural properties which define how the structure will react to certain loading conditions. The stresses travel from node to node and are analyzed to see how much stress/strain that section observes. FEA was mostly used on geometries that are too complicated to solve by hand, this was especially true with the aerospace industry. The industry cannot afford to make a scaled or actual model of every aircraft design because it was too expensive to do. FEA was far cheaper to use because it testing and results are from computer simulations. 


\subsection{Literature Review}

\subsubsection{Composite Sandwiches with through holes with a shear key}

In 2009, a research on damage arrestment devices was conducted by a group of California Polytechnic State University, San Luis Obispo students for their senior project on how the devices prevent a crack or failure from propagating any further from a through hole on a composite sandwich panel ${ }^{\mathrm{ii}}$. The researchers were Balatbat, Dixon, Jacobson, Mackey and Woo.

The specimens were foam panels placed between skins of fiberglass with center holes and were manufactured through the vacuum resin infusion process (VRI). Some of the specimens contained damage arrestment devices surrounding the hole; this can be seen in Figure 11. Other specimens contained straight damage arrestment devices on either one side, or both sides of the part. Some improvement in stiffness and ultimate strength were seen from the circular damage arrestment devices in specimens containing holes with diameters equal to or larger than half of the width of the part (3 in and 4 in hole diameter specimens). Circular damage arrestment devices were successful in instigating a failure on the side of the part that did not contain the damage arrestment device, which made the failure more predictable and easier to study and understand. Straight damage arrestment devices also made the failure occur away from the center hole at the end of the damage arrestment device.

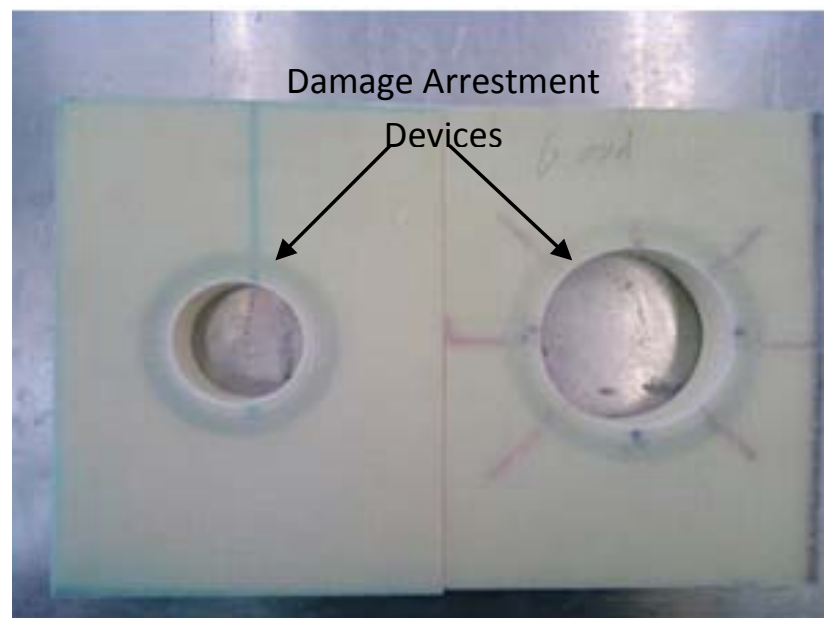

Figure 11: Senior Project Test Pieces 
The maximum loads among the specimens were compared, as shown in Figure 12. The figure shows that the control group piece, a panel without any damage arrestment devices or holes, was able to take the most force before failing. The trend shows that as the hole diameter increased, the lower the ultimate load the panel could take. The trend also shows that the damage arrestment devices helped the material compared to parts with holes and no damage arrestment devices. The most significant increase was seen in the 3” and 4” hole diameters. The trend also showed that specimens with a small diameter hole were weaker with damage arrestment devices than without.

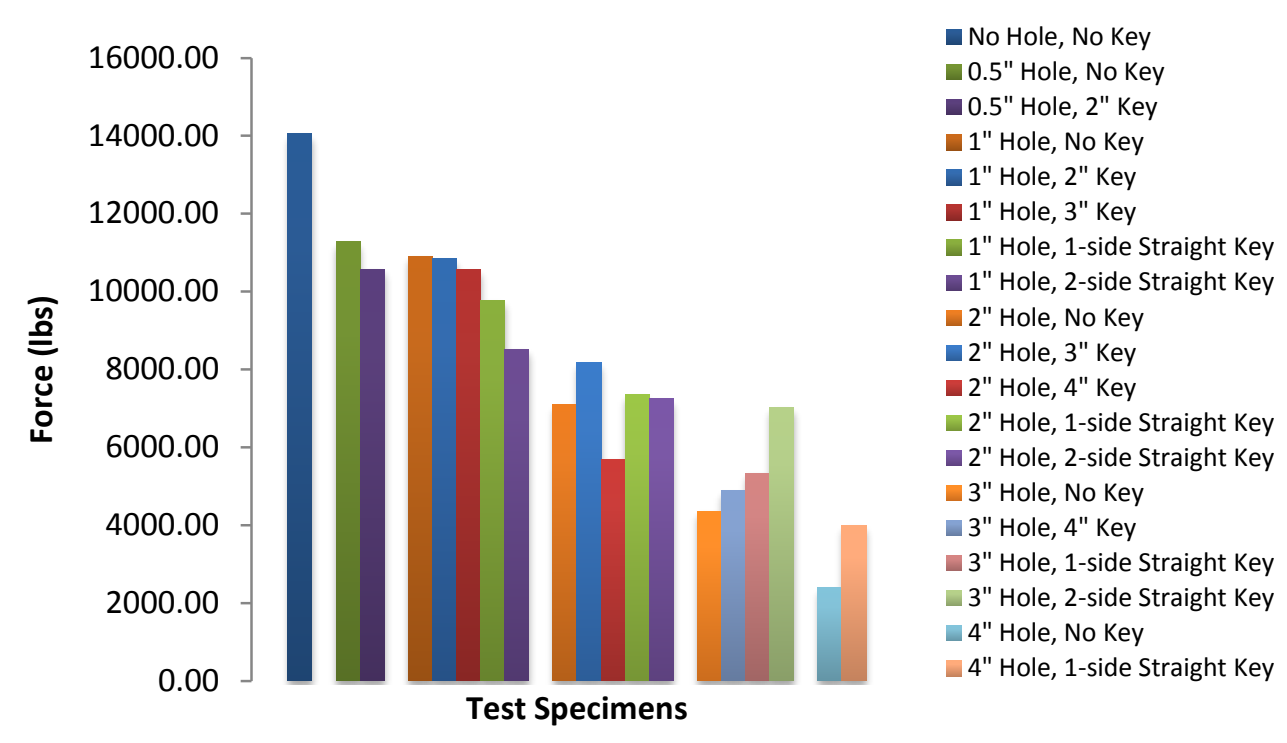

Figure 12: Ultimate Load Comparison

\subsubsection{Fastener Failure Interaction of Composite Sandwich Panels}

In 1996, a research on fastener/hole interaction with composite sandwich panels was investigated by Camanho and Matthews ${ }^{\mathrm{iii}}$. The two did experimental research on seeing different variations with how the fastener affects the composite sandwich panel. They observed the effects of joint geometry, ply-orientation, and lay-up and through-thickness pressure on the joint connection for both single and multi-fastener panels. The research found that there were four types of failure modes found during the testing, as seen in Figure 13. 


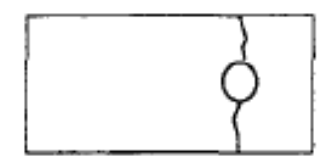

Tension

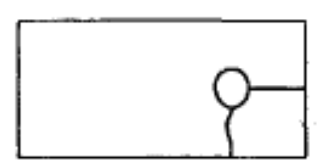

Cleavage

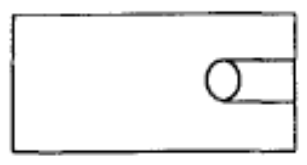

Shear

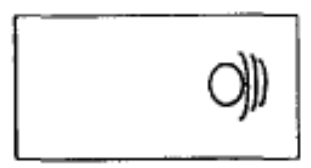

Bearing

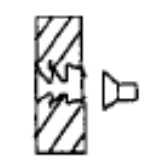

Pull-through

Figure 13: Examples of Failure Modes for Fastener/Joint Interaction

The four failure modes observed during the experimental testing were tension, shear, cleavage, and bearing. Tension failure was when failure was seen along the entire width of the strip/panel due to the bolt diameter being a huge portion of the overall width of the strip/panel. Bearing failure was when the hole was elongated due to the fastener interaction; this was due to the bolt diameter being a small fraction of the panel width. Shear failure was considered a specific case of bearing failure, this occurring when the hole was too far from the edge of the strip/panel. Cleavage failures are a combination of bearing and tension failure.

Camanho and Matthews also observed that there was a "sweet spot" where the right hole diameter/part width ratio and the distance of the hole from the edge of the part would give a perfect bearing failure, this can be seen in Figure 14. The figure shows the empirical observation of hole placement and diameter in respect to the ply orientation of the panel. From both graphs, it can observed that as the width to diameter ratio increases, the tensile stress concentration factor, $\mathrm{K}_{\mathrm{tb}}$, and the shear stress concentration factor, $\mathrm{K}_{\mathrm{sb}}$, becomes asymptotic. The factors are $\mathrm{a}$ relationship of the max hoop and shear stress over the desired bearing stress of the part. 

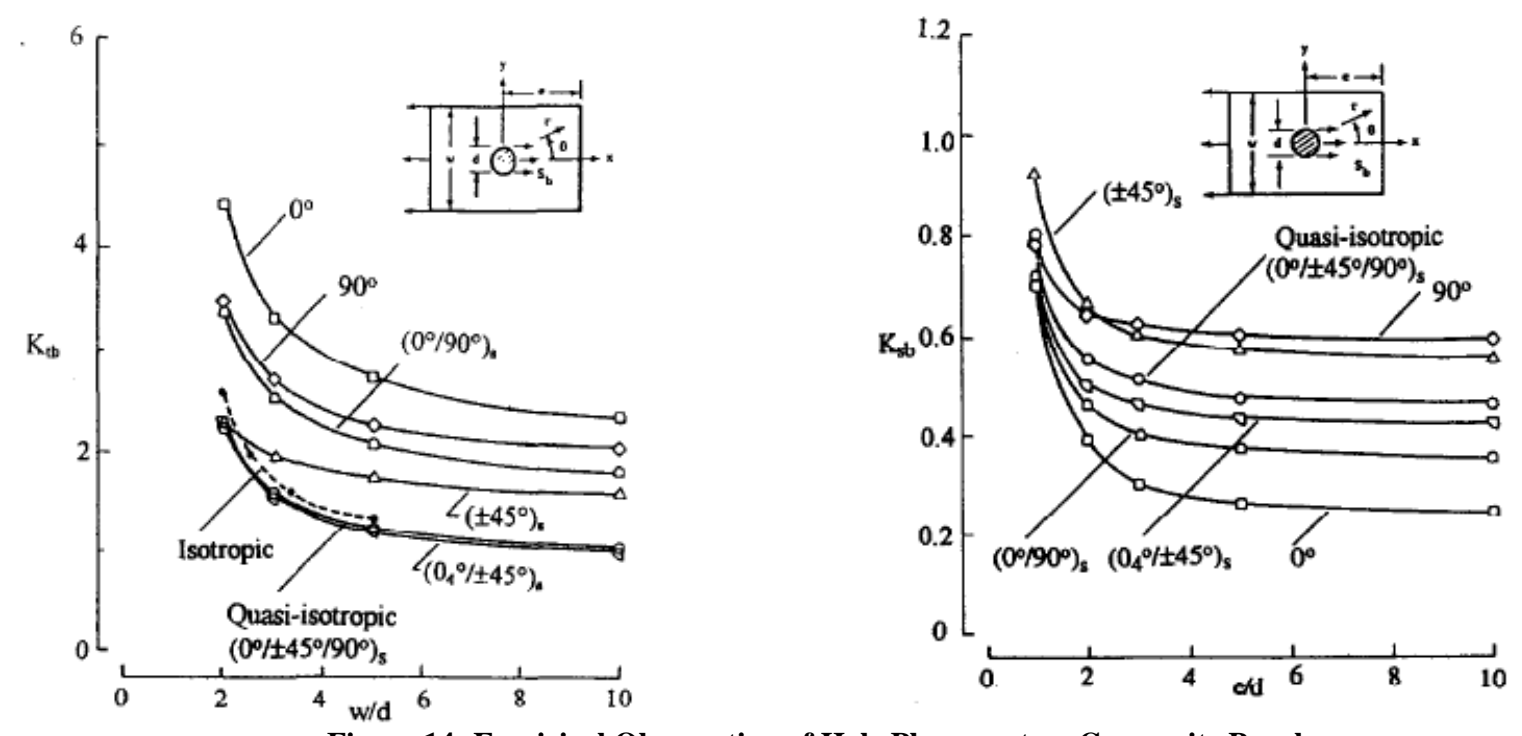

Figure 14: Empirical Observation of Hole Placement on Composite Panel

\subsubsection{Monotonic and Dynamic Loading of the Composite Sandwich}

In the same manner as Camanho and Matthews, another experiment on composite sandwich panels with fastener interactions was investigated in 2001 with the use of metal jigs under monotonic and dynamic loading ${ }^{\text {iv }}$. Demelio and others found that the first problem with composite testing was that drilling holes in composite panels could cause delamination of the face sheet and the fraying of fibers along the hole. The researchers found out that drilling with a slow speed rate and having a metal plate behind the panel would decrease burrs and prevent the face sheet from delamination.

The static and fatigue testing was varied with a carbon/glass and carbon/Kevlar laminate with four different types of fasteners. They conducted the static and fatigue testing under two types of loading a pull-out loading and a shear loading. For the purposes of the previous works talked about, the shear loading testing and results was discussed. The specimens were tested by bolting a composite sandwich panel to two rectangular metal plates that were connected to a testing machine; this can be seen in Figure 15. The loading from the machine was a tension force 
with an extensometer attached between the steel plates to measure the strain of the sandwich composite panel due to the shear loading.
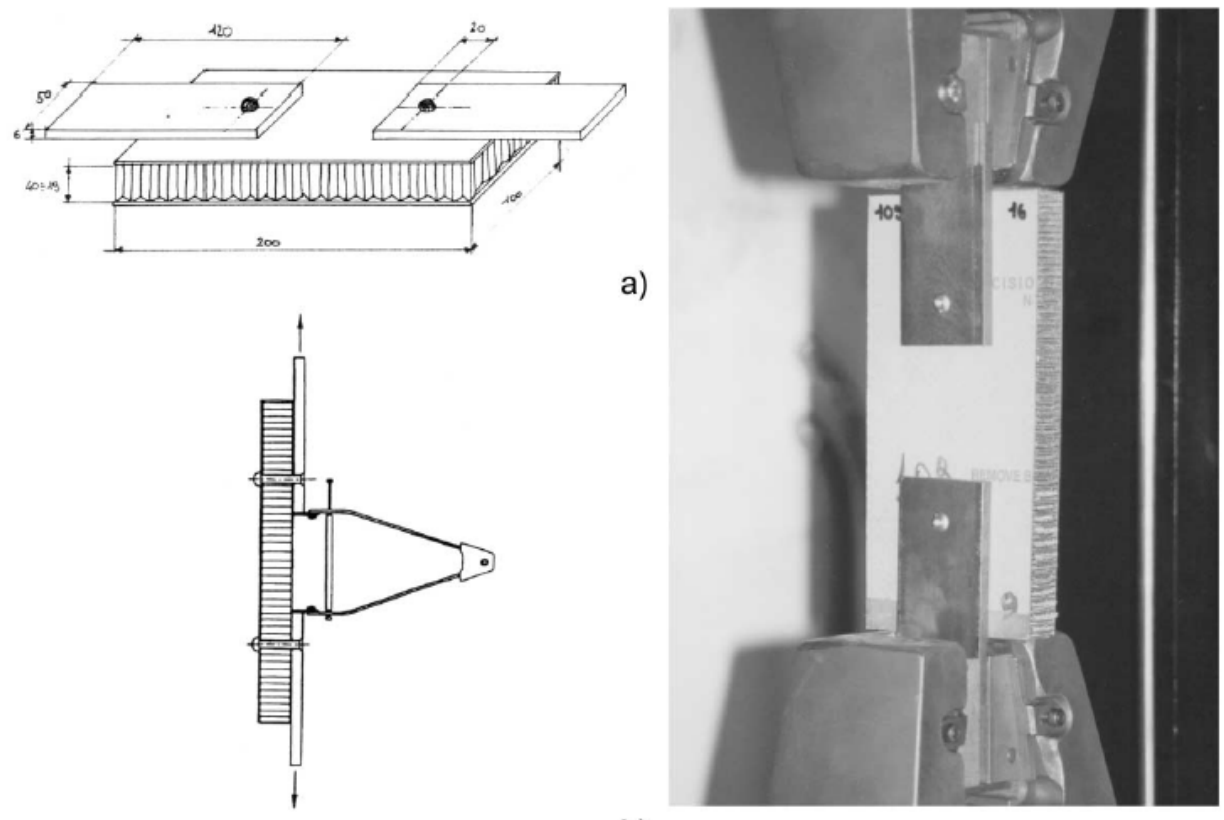

a)

b)

c)

Figure 15: Shear Testing by Demelio et al

The conclusions that were observed from the experiment were that under shear loading the fasteners had a higher failure loading than the pull-out loading. This could be due to the piece preventing the fastener from completely shearing through the piece. They were also able to observe the types of failure that occurred around the fastener/panel joint. Bearing stress was mainly observed by elongating the hole and fraying the face sheet from the panel; this can be seen in Figure 16. A trend for the load/extension curve of the shearing specimens was observed. They first observed that the curve had a first significant line that related to the structure's settling over the load. The next line, the slope, rapidly increased until it reached the maximum value of the fastener/panel joint interaction. The final line occurred when the load dropped down as the fastener first widened the hole until it crushed the skin and completely left the panel. 

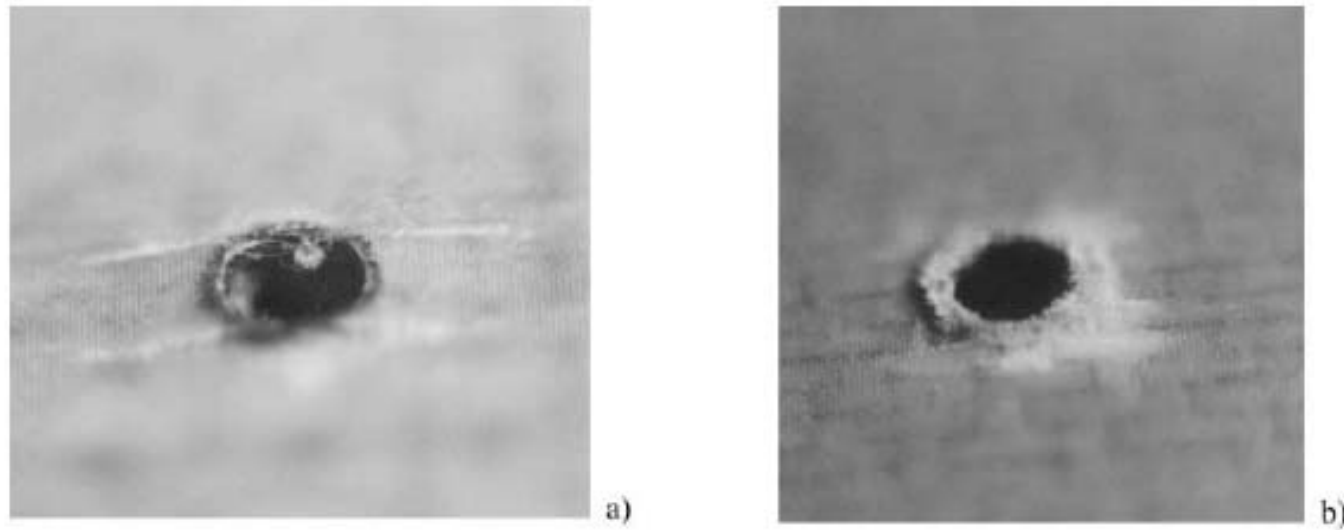

Figure 16: Types of failure seen during testing by Demelio et al

For fatigue testing, the results were inconclusive by being too spread out and having very small values to formulate any valid trend for the data. The observation from the fatigue testing was that there was a $20 \%$ band where specimens or systems can be useful for the fatigue design analysis for this type of fastener/panel set-up.

\subsubsection{Insert with a bolt Joint}

Instead of studying how fasteners affect composite sandwiches, another research was conducted in 2008 to study the insert joint strength of composite sandwich structures ${ }^{\mathrm{v}}$, by Song et al. The paper addresses and experimental study of the pull-out and shear failure loads of composite sandwich insert joints. The composite sandwich panels consisted of Nomex honeycomb core and a carbon-epoxy composite with a film-type adhesive to help bond the core and face sheet. The specimens were varied depending on the core height and density, face thickness, insert clearance, and loading direction. The results showed that pull-out loading was affected by the properties of the core, but also by the thickness of the face sheet. Specimens with and without potted inserts showed significant differences under shear loading, potted inserts had higher failure loads than tightly fitted insert joints. The results showed that for shear loading, can be seen in Figure 17, the failure loads of joints were dominated by the face sheet thickness, while core properties had little to no effect on the failure load. 

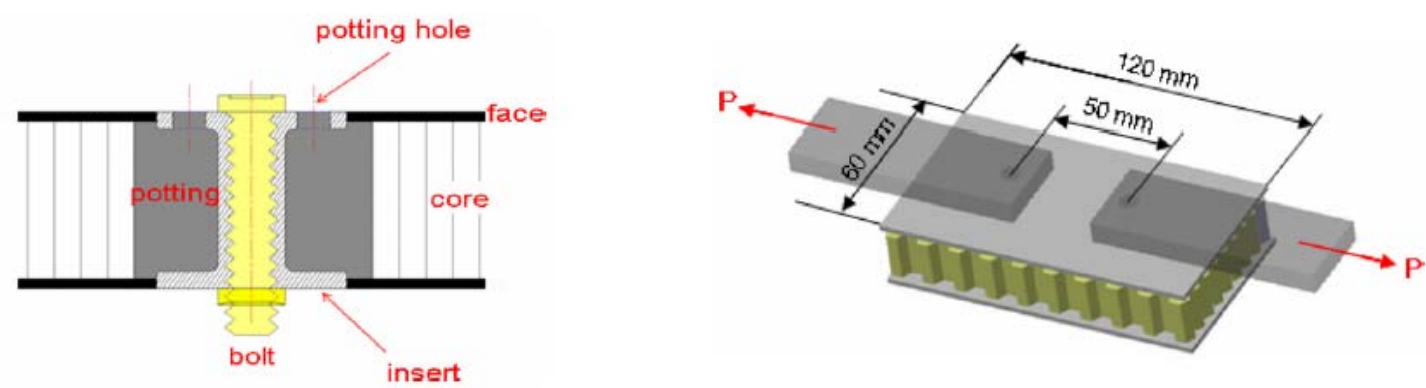

Figure 17: Testing Apparatus by Song et Al

For the experimental testing, Song et al, defined the failure load for both types of testing as the first peak of a load-displacement curve, also known as the yield load of the specimen. Once the specimen reached the failure mode, the separation of the potting material and the core was developed. The core beneath the composite face cannot support the insert any longer and all pullout of the insert was supported by the face sheet. At this point, it indicates the final failure of the composite sandwich; this can be seen in Figure 18.
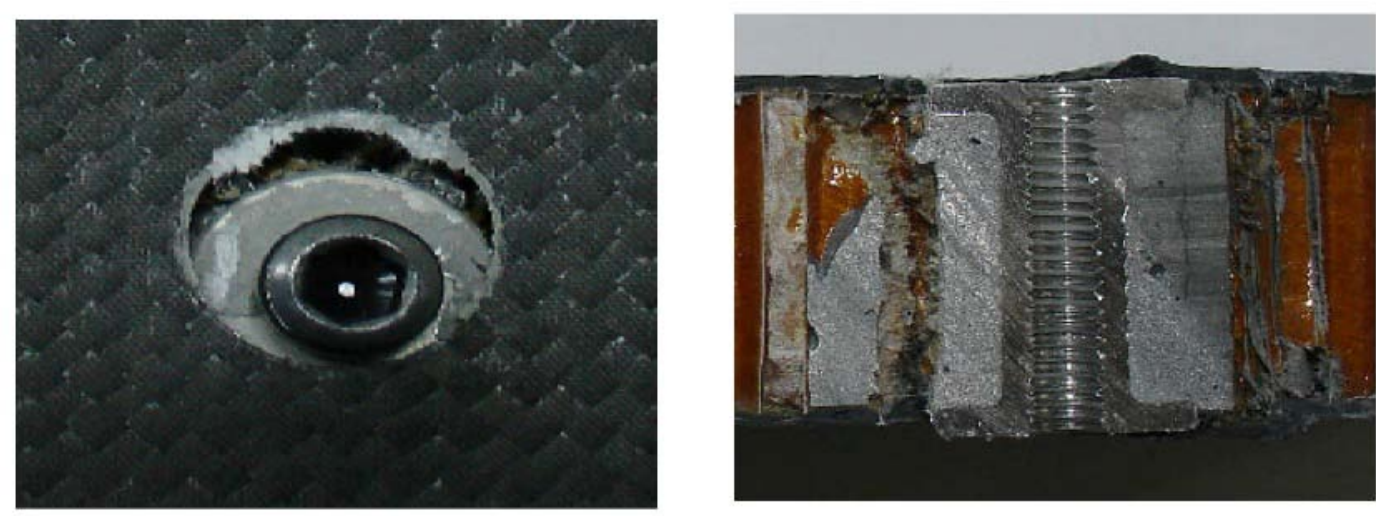

Figure 18: Failure of potted insert specimens by Song et al

\subsubsection{Finite Element Models of Composite/Fastener Interactions}

In 2004, a study on the finite element model of a single-lap, single-bolt composite joint was developed by McCarthy et $\mathrm{al}^{\mathrm{vi}}$. A validation of the finite element analysis solution was compared to experimental results that were conducted by the researchers. The model has been 
developed for a study of the effects of bolt-hole clearance, without any experimental testing, just all numerical results. McCarthy et al were the first researchers to publish a paper on threedimensional finite element modeling on variable hole clearance of composite laminates.

The single-bolt composite joint was modeled using MSC. Marc. Efficiency was improved by defining contact bodies as sub-parts of the joint components, and using a contact table to define which contact bodies could come into contact. The use of single-sided contact placed restrictions on the meshing of the different joint parts, and the order in which contact bodies were defined. The mesh also had to be adjusted to minimize passing through of “overhanging nodes”. Finally, it was found to be vital to choose the analytical contact option, which fits a smooth surface through the contact body; this can be seen in Figure 19.
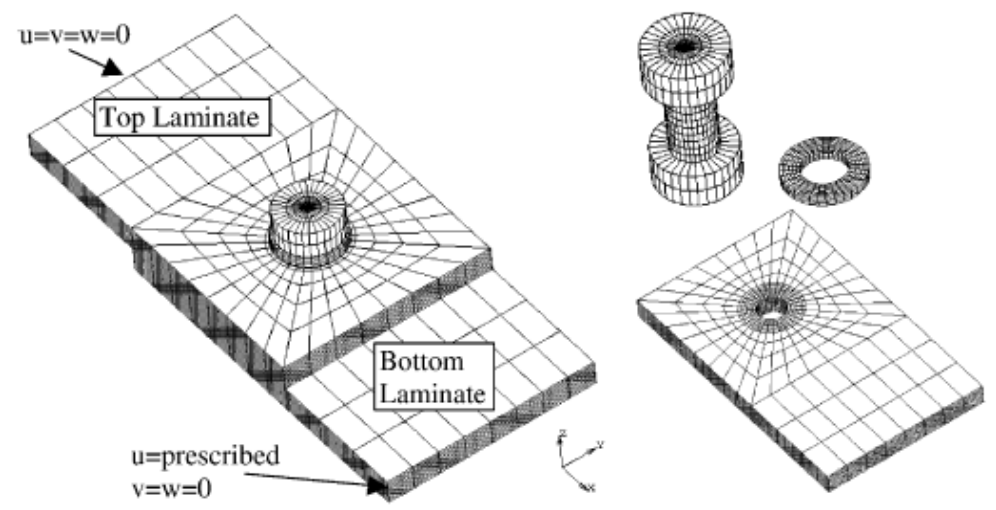

Figure 19: FEM Model of McCarthy et al

Strain gauges were placed on a number of joints and the resulting effects were found both experimentally and numerically. Results showed that significant amounts of bending of the laminates, so that the external surface of the joint was in compression, despite the tensile loading applied to the joint. An observation seen during the research was that the surface had a doublecurvature, meaning that the joint was saddling like a wide beam in bending. The joint was also found to twist slightly about its longitudinal axis. The strain along the surface of the laminate was found to not be affected by the bolt-hole clearance, except located on the loaded side of the hole. 
A parametric study was carried out during the research to see the correlation between the numerical and experimental solutions without incurring an excessive penalty on computational resources. From McCarthy et al., "the factors that most improved the model were a refined nonoverlap region, use of the assumed strain formulation with first-order elements, implementing a routine to allow separate tensile and compressive properties, and modeling the clamped area of the joint.” These factors from the research showed a significant improvement between the experimental and numerical strains at the axial joint location, with little increase in computational resources.

For the finite element model, the stress at and around the hole was a major concern. It was important to recognize the presence of singularities at this location in the model. Singularities are also seen in the model, such as the washer-bolt, washer laminate, and bolt-laminate interfaces, and interfaces between plies. Great care and refinement are needed around these locations to accurately determine the failure criteria or stress concentration factors.

As the bolt rotation increased, the clearance results of the joint decreased in bolt-hole contact area and decreased in joint stiffness. The clearance trend of joints gradually increases in stiffness with an increase of applied loading. The trends show that three-dimensional finite element analysis was capable of quantifying these effects with a high degree of accuracy in comparison of experimental data.

The stress distribution in the laminate of single-lap joints shows a non-uniform trend through the thickness of the laminate and the way the laminate was laid-up. An increase of radial stress was shown in the clearance when homogenous material properties were used for the model, which showed a shift of the location of the peak tangential stress towards the bearing plane, an increase of the tangential stress and the compressive tangential stress of the bearing plane. With the use of layered solid elements, the radial and tangential stress in each ply of the laminate was recovered. The recovery of the stresses was consistent with previous two-dimensional studies on the clearance of laminates. 


\subsection{Scope of Work}

The research encompassed the use of composite sandwich panels with holes drilled into it. Fastener or bolts were placed through the hole to be used to imitate the panel being attached or joined with another panel; this can be seen in Figure 20. The main purpose of the research was to investigate the effects of a fastener/joint interaction with a composite sandwich panel, with emphases on how the fastener affects the failure mode around the hole section of the composite sandwich panel.

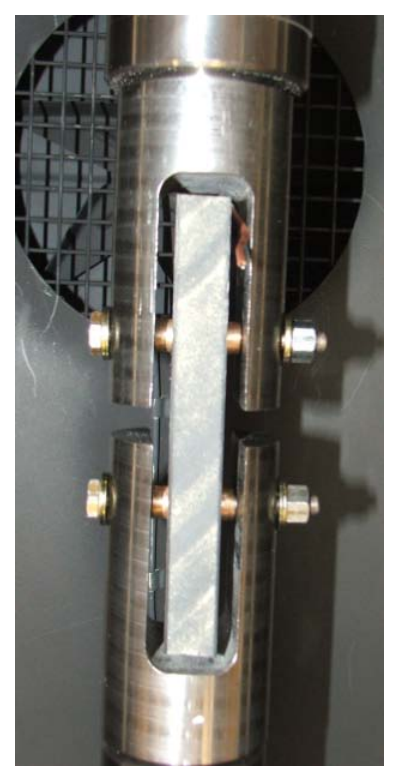

Figure 20: Example of experimental testing

The research was also to prove that adding a damage arrestment device (DAD) around the hole of the composite sandwich panel would prolong the failure by increasing the stiffness and the strength of the panel. The thickness of the DAD was varied to see how it affects the failure of the panel and to see if the thickness affects the overall stiffness and strength of the material. The testing consisted of monotonic and fatigue loading. The monotonic loading was used to investigate the failure mode and the material characteristics of the composite sandwich panel. The fatigue loading were used to investigate how the DADs affect the overall life cycle of the composite sandwich panel for future design and application of the concept. 
The analytical analyses were performed by modeling the composite sandwich panel with Finite Element Modeling (FEM). The FEM was used to determine the failure mode and the displacement around the hole of the composite sandwich panel. The experimental and analytical methods were then compared with one another. From this investigation of various composite sandwich panels for a hole and fastener, a determination was made of the most optimum design of a DAD to the panel.

The paper discussed the experimental work for the research’s experimental design. The evolutionary design of the test specimen was discussed from it preliminary dimension and manufacturing process to its final dimension and manufacturing process. The evolutionary design of the DADS was also discussed. The problems that arose during the design process, such as the test apparatus, the manufacturing process, and the prepping of the specimens, were discussed on how they affected the final design of the experiment.

The paper discusses the detailed and step by step process of manufacturing and preparing of the test specimen. The manufacturing's of test specimens with and without DADs was discussed from the cutting of the foam and composite materials to cutting down the panels to dimensions and the marking and naming of the test specimens. The manufacturing and testing process for the volume fraction and material properties of the test specimens were discussed. The experimental set-up and testing of the research was then designed. The strain rate to run the speed of test of the Instron machine for all monotonic testing was investigated. The failure criterion for monotonic loading was also decided for consistency between the results. The design of the fatigue testing for the research was conducted such as the failure criterion and the frequency of the machine. Lastly the procedure for setting up and running the experimental test was discussed.

The paper also discussed the theoretical analysis of composite sandwich panels such as determining the volume fraction of the face sheets. The volume fraction determines if the matrix to fiber ratio of the face sheet was ideal enough that the laminate wasn't too brittle or too ductile 
for usage. An analysis on the overall Elastic Modulus of the composite sandwich panel was determined.

The experimental and numerical results of the research were discussed in the paper. All experimental testing pertaining to the variation of thickness of the DADs and the overall life cycle of the composite sandwich panels were discussed. All numerical modeling was addressed in full detail, such as how they were modeled and meshed, to how the boundary conditions and the loading were applied to the model. The results of the numerical models such as the failure modes and the maximum displacements for each model were discussed. Lastly, a comparison between the experimental and numerical results was examined in detail and the effectiveness of the DADs to the composite sandwich panel was examined. 


\section{Manufacture of the Test Specimen}

The design of the experiment was discussed in this chapter. The evolutionary design of the test specimen was discussed from its preliminary dimension and manufacturing process to its final design. The evolutionary design of the DADs was discussed. The problems that arose during the design process, such as the test apparatus, the manufacturing process, and the prepping of the specimens, are discussed on how they affected the final design of the experiment.

\subsection{Testing Standards}

Since there are no definite or accepted standards to test composite sandwich panels with a fastener, a variation or evolution of some of the standards was used for the experimental testing to require the desired or predicted results of the research.

ASTM C364/C364M was the standard test method for edgewise compressive strength of sandwich composites. The test method subjects a composite sandwich panel to a monotonically increasing compressive force along the face sheet of the sandwich. The force was transmitted to the composite sandwich panel through either clamping or bonding end supports to the machine. The data collected was presented in terms of the nominal cross-sectional area of the face sheet, not including the core. The standard also defines that a test was only successful if the failure of the specimen occurs away from the supported ends. It also mentions that the column would see a buckling type of failure if the columns are too long, thus creating a set of parameters for the panel design to avoid the failure; this can be seen in Figure 21. The standard defines the dimensions based on the thickness of the face sheet since it will carry most of the loads due to being significantly stronger than the core. The standard also defines that a minimum of five specimens must be tested per case unless the experiment was designed to a desired sampling error. It also suggests that the loading rate of the machine should be on the user's discretion, but recommends that the testing should be conducted within three to six minutes to reach the failure mode. 


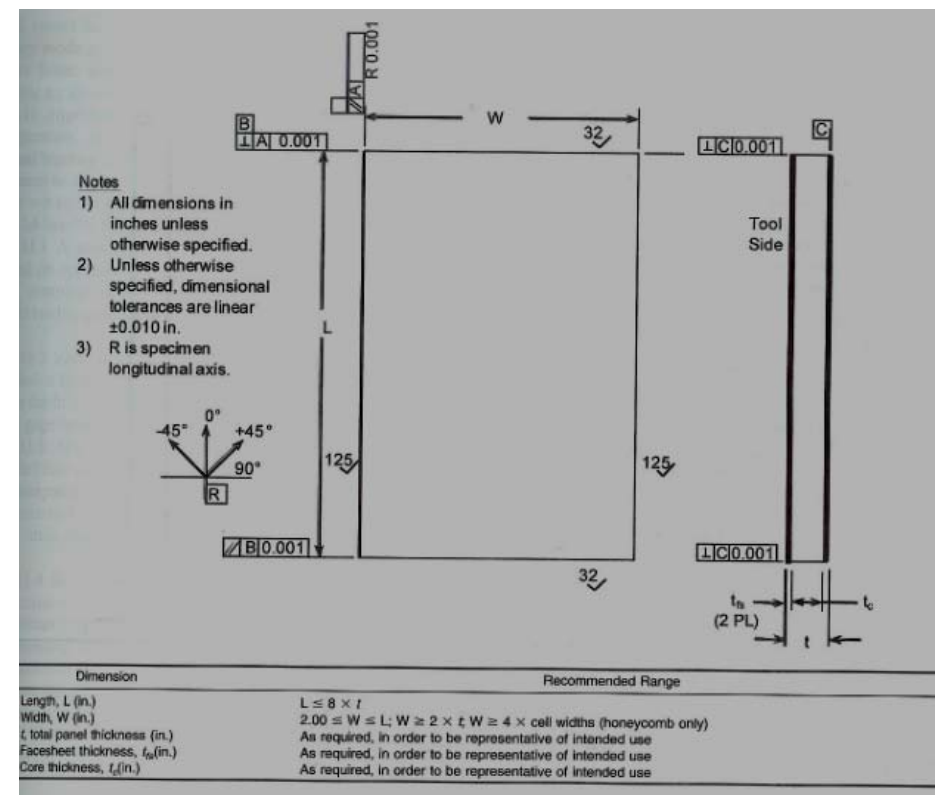

Figure 21: ASTM C364/C364M Test Specimen Dimensions

Another standard was ASTM D3410/D3410M which determines the in-plane compressive properties of composite sandwich panels with the use of compressive forces from shear loading. The purpose of the standard was to find the material characteristics of the sandwich composite material, such as ultimate compressive strength, ultimate compressive strain, and compressive modulus of elasticity, Poisson's ratio, and the transition points of the test piece. The standard follows the same specifications from the previous standard stated, but the difference was the loading seen on the part.

A testing standard for composite structure interaction with fasteners was ASTM D5961/D5961M-05. The purpose of the standard was the bearing response of composite laminates under a shear loading. The bearing load was applied by pulling the specimen in tension or compression through a torque fastener or pin with a diameter of 6 millimeters. The testing apparatus consist of a metal jig that was clamped to one side of the machine while the laminate was bolted to it and clamped to the other, this can be seen in Figure 22. The specimens would have one of the four failures that were previously discussed in the literature review. 


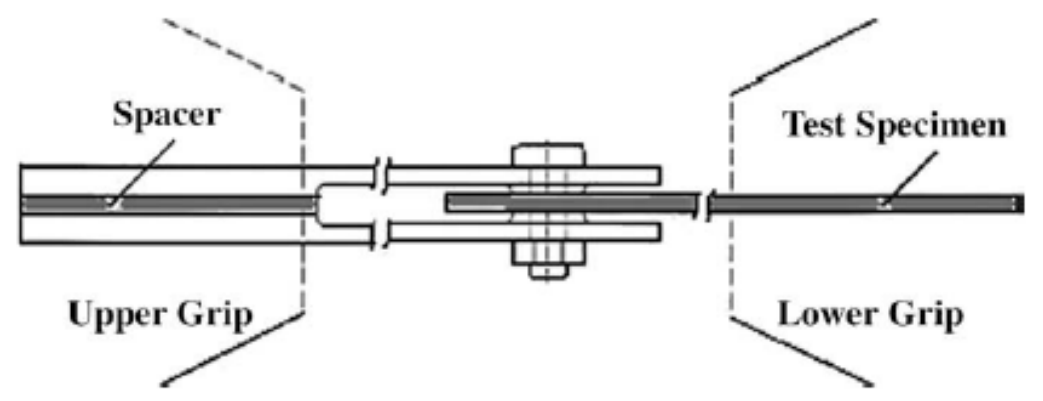

Figure 22: ASTM D5961 Testing Apparatus

With the use of the previous standard, ASTM D68873-03 may be used in conjunction with ASTM D596; the bearing fatigue characteristics of the composite laminate can be determined by subjecting it to a cyclic bearing loading. The testing consist of having a repetitive constant amplitude force, cycled at a specific frequency. The cyclic loading could be tensiontension, compression-compression, and tension-compression. The failure mode for the fatigue testing could be based off a desired bearing stress or based on a certain number of fatigue cycles to measure the bearing stress.

\subsection{Material Selection and Characteristics/Properties}

Since this research was a derivative of the work done by Balatbat et al, a sandwich composite panel was used. The composite sandwich panel consisted of a foam core with fiberglass as the face sheet. Due to the lessons that were learned, the decision was made that the foam core must be heat resistant to withstand the temperatures seen during curing time and testing. This was due to the hazardous nature of non-heat resistant foam because it was harmful to the researchers' health by releasing poisonous fumes. After contacting plastic manufacturers, it was decided to use Last-A-Foam FR-6710 from General Plastics; this can be seen in Figure 23. The foam was polyurethane closed cell foam with a density of $10 \mathrm{lbs} / \mathrm{ft}^{3}$ that has been certified by the FAA to withstand temperatures up to $275^{\circ} \mathrm{F}$. The foam was used for honeycomb edge closeout for aircraft interior sandwich panels, models and design prototypes, vacuum form dies, 
hazardous materials transport packages, and insulated structural panels. For aircraft use, the foam was used for overhead storage bins, passenger cabin class dividers, galleys and lavatories. The selection of the foam was more of an arbitrary decision with the main factors being monetary. The foam was the least expensive of researched foams that was heat resistant.

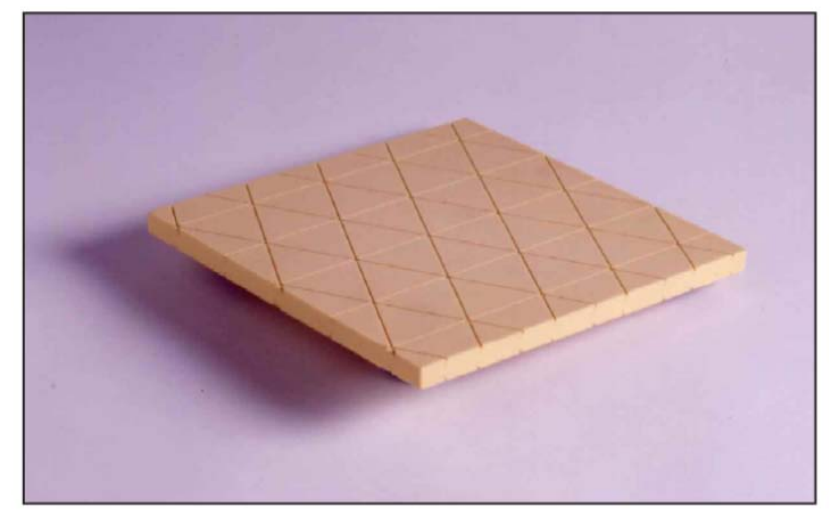

Figure 23: Last-A-Foam FR-6710

The foam's material property was obtained from the manufacturer and was tested to determine the accuracy of the data from the manufacturer; this can be seen in Table 9 in 6.1.2 Material Properties. The experimental testing for the material properties was conducted using ASTM C364/C364M procedure. From the table, the material properties of the foam decreased by $20.6 \%$ for the compressive strength and about $34.8 \%$ for the elastic modulus of the material. The decrease in compressive strength and elastic modulus could be due to the way the specimens were tested. The testing procedure for the foam can be different from how the manufacturer tested the foam causing the significant difference in material characteristics. The decrease in material property can also be due to the degradation of the material from being stored too long after purchasing and receiving the material. Being stored in an uncontrolled environment can affect the material characteristics of the foam; for example, storing the foam in the sun would make it susceptible to UV degradation. For comparison with the FEM, the experimental results of the material characteristics were used for comparison between experimental and numerical solution. 
For the face sheet selection, the composite sandwich panels were made out of low temperature curing epoxy prepreg matrix resin known as LTM45, which was manufactured by The Advanced Composites Group; this can be seen in Figure 24. The carbon epoxy sheets are a bi-directional weave with an epoxy/resin pre-impregnated within the weave. From the previous research, fiberglass was used for the sandwich composite panel. A move from fiberglass to carbon fiber was due to the more prevalent use of the composite in industry. Another main reason was that during the literary review, it was found out that carbon fiber was the preferred use to study the effects of fastener interactions with composite sandwich panels. This was primarily due to the fact that carbon fiber was a lot stronger than fiberglass with no significant increase in the weight of the composite. Also, the main driver for choosing the material was the abundance of pre-preg carbon fiber that the Cal Poly Aerospace Engineering Structures/Composite Lab has. The same reason for the selection of the foam was used for the selection of the composite material because the researchers did not have to spend any extra money for the material.

The main advantages of LTM45 are that the material was suitable for autoclave, vacuum bag or press moldings. Some main features are that it only needs a low temperature to cure the material $\left(131^{\circ} \mathrm{F}\right)$ and were suitable for high temperature use $\left(356{ }^{\circ} \mathrm{F}\right)$. The material was ideal for minimum cost prototype and short production run parts from lost cost molds. It also can give more accuracy in moldings compared to conventional high temperature cure prepreg systems.

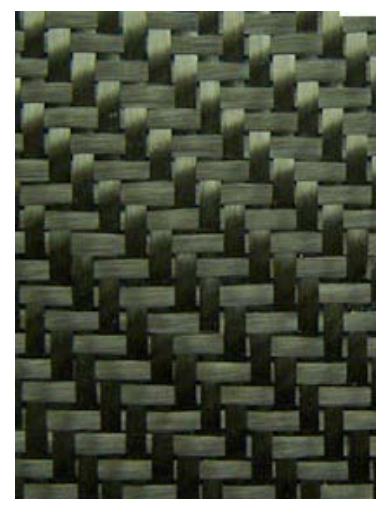

Figure 24: LTM45 Carbon Fiber 
Similar to the determination of the material characteristics of foam, the material properties of LTM45 were obtained from the manufacturer and tested to validate the data, this can be seen in Table 10 in 6.1.2 Material Properties. From the table, the material properties of the material decreased by $35.0 \%$ for the compressive strength and about $58.6 \%$ for the elastic modulus of the material. This decrease in material property can be due to the degradation of the material. The material was donated by Boeing over three years ago and was stored in a freezer that barely met the cooling standards of the carbon. When the material was donated from Boeing, it was considered by the FAA to not meet its specifications, but it was still suitable for research usage. The values obtained from experimental testing show that the material properties of the LTM45 are still adequate for the research because compared to the foam the material was infinitely stiff and can handle the objectives of the thesis. Just like the foam, the experimental results of LTM45 were used for the material characteristics for the FEM.

A film adhesive, known as LTA45ELNC, was used between the face sheet and the core to give the face sheet a better bond to the core. The usage of film adhesive was typical in industry because pre-preg only contains an enough resin content to fully cure the composite fiber instead of it having enough to cure and bond to something at the same time. The material characteristics of the film adhesive were not investigated because it was assumed that the film adhesive was fused with the pre-preg and any excess film adhesive was pushed out of the plate. The decision to add film adhesive will be further discussed in 2.4 Specimen Design.

For the fastener, aircraft grade bolts, washers, and nuts were used for the experiment. The bolt, AN4-24A, was certified by the FAA. The dimensions of the bolt are 2.53125 ” in overall length with a thread length of 2.0625” and a diameter of 0.25 ”. The bolts are made out of 8740 steel with a minimum tensile strength of 125,000 psi. An oilite impregnated bronze bushing was also used in between the composite panel and the bolt to increase the pressure distribution along the hole. The bushing has an inner diameter of 0.25 ” and an outer diameter of 0.375 ” with a 
length of 1”. For the FEM, 8740 steel was used as the material properties of the bolt and bronze for the material properties of the bushing.

\subsection{Manufacturing Process}

For the manufacturing of the panels a Tetrahedron Press was used to cure the LTM45 prepreg; which can be seen in Figure 25. The choice of manufacturing of the face sheet was between the Tetrahedron Press and an autoclave. An autoclave would give a better pressure distribution than the press by applying it on all six surfaces rather than two. It was also better because it was a sealed and controlled environment that can maximize the curing of the face sheets. The press, on the other hand, was less complicated to operate and would apply pressure and heat directly on the face sheet instead of the core. This would make the face sheet more brittle than using the autoclave. The decision to use the press over the autoclave, even though the autoclave was the better choice to manufacture the panels, was due to the operation and maintenance cost of the autoclave. With the limited funds for the research, the press seemed a better process.

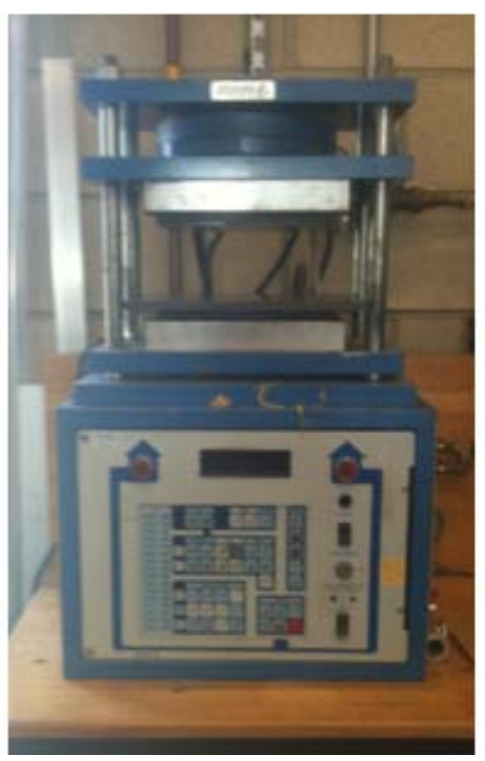

Figure 25: Tetrahedron Press 


\subsection{Specimen Design}

The preliminary design of the composite sandwich panel was based on the dimensions from ASTM D5961/D5961M on the standard test method for bearing response of polymer matrix composite laminates. The standard was applied to a sandwich composite panel instead of the specified laminate because of a lack of standard to investigate the bearing response of composite sandwich panels. The standard defines the dimensions of the laminate based on the thickness of the laminate and the diameter of the hole; this can be seen in Table 1. The dimensions for the specimen thickness were ignored due to the set thickness of the core and the face sheet. The other dimensions such as width, length, and hole edge distance were used from the standard based on a selected hole diameter. The standard does not set concrete dimensions, but sets guidelines to make results comparable to other researches.

Table 1: ASTM D5961/D5961M Specimen Dimensions

\begin{tabular}{|c|c|}
\hline Specimen Thickness & $<$ Hole Diameter/2 \\
\hline Specimen Width & $<6$ x Hole Diameter \\
\hline Specimen Length & $<7.5 ”$ \\
\hline Specimen Hole Edge Distance Ratio & $>3 \times$ Hole Diameter \\
\hline
\end{tabular}

A preliminary specimen dimensions was designed, this can be seen in Figure 26. An initial bolt diameter of 0.25 ” was selected for the diameter of the fastener. This was chosen arbitrarily for being the second smallest bolt diameter that can be purchased off the shelf. Another reason the diameter was arbitrarily chosen was because the researchers wanted to minimize the specimen dimensions as much as possible due to the dimensions being dependent on the bolt diameter. This would lessen the usage of resources and save money. 


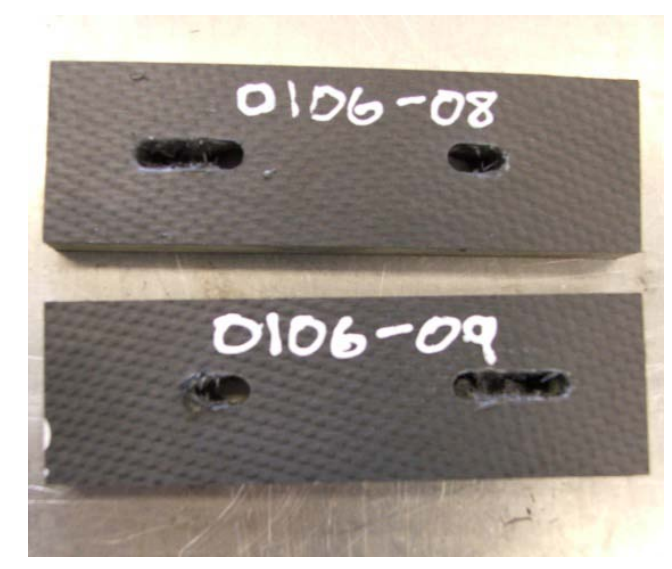

Figure 26: Preliminary Specimen

The preliminary specimen dimensions were a length of 5.0”, a width of 1.5”, and the thickness was dependent on the number of laminates the faces sheet contained. The thickness of the core or the foam was set by prices from the foam manufacturer. The smaller the thickness, the more accurate the manufacturer needed to be, meaning that the price per sheet increased. The researchers settled on a thickness of 0.5 ” for the foam due to the price meeting budget demands. This thickness also allowed the researchers to investigate more layers for the DADs, making it okay to study the effect of the DADs on the core. An arbitrary selection of two layers of LTM45 was chosen for the face sheet. An assumption was made that two layers of LTM45 on each side of the core would give infinite stiffness to the core due to the elastic modulus of the face sheet being 975 times higher than the core. With a thickness of 0.013” per sheet of LTM45 and a thickness of 0.5 ” for the foam, the total thickness for the specimen was 0.552 ”. The final dimension of the preliminary specimens came out to 5 ” x 1.5 ” x 0.552 ”. The specimens contained two holes due to the loading from the test jigs; this will be discussed further in 2.6 Testing Apparatus Design. The hole locations were similar for both holes, with an edge distance of 1.5” and a distance of 2.0" from one another. The edge distance met the requirements set from the standard by being more than three times the diameter of the hole. The preliminary specimen dimensions are summarized in Table 2. 
Table 2: Preliminary Specimen Dimensions

\begin{tabular}{|l|c|}
\hline Bolt Diameter & $0.25 "$ \\
\hline Bolt Location from edge & $1.5 "$ \\
\hline Thickness of foam & $0.05^{\prime \prime}$ \\
\hline Thickness of each face sheet & $0.026 "$ \\
\hline Specimen Length & $5.0 "$ \\
\hline Specimen Width & $1.5 "$ \\
\hline Specimen Thickness & $0.552^{\prime \prime}$ \\
\hline
\end{tabular}

The preliminary specimens were then tested and analyzed on the viability of the design. The results showed that the bolt interaction with the specimen was showing bearing stress that was discussed earlier and meets the objective of the thesis. The failure of the specimens was desired but unseen problems were encountered during the testing that resulted in a redesign of the test pieces. The first problem was that the hole diameter needed to be enlarged to accommodate a bushing to evenly distribute the load along the hole. The bolt started to bend under the loading from the jig, causing a concentrated load distribution on the face sheets instead of the foam. The increase in hole diameter would increase the width of the part. The second problem was that the initial jig was torque, adding a bending load on the specimens instead of pure axial loading. This will be discussed in 2.6 Testing Apparatus Design. The final problem that was encountered was that a film adhesive needed to be added between the core and face sheet to prevent delamination of the face sheet under prepping and testing. The face sheets could easily delaminate from the core from prepping the panels such as cutting to dimension or from drilling; this can be seen in Figure 27. The figure shows that the face sheet can easily be removed from the core with little effort and shows that a better bond was needed between the two surfaces. As discussed earlier, drilling through any composite was challenging because, depending on several parameters, the face sheet can cause delamination between the core and face sheet; this was further discussed in 2.7 Hole Drilling Optimization. Another driving factor to add the film adhesive was that the face sheet was easily delaminating when the fasteners were tightened before testing. 


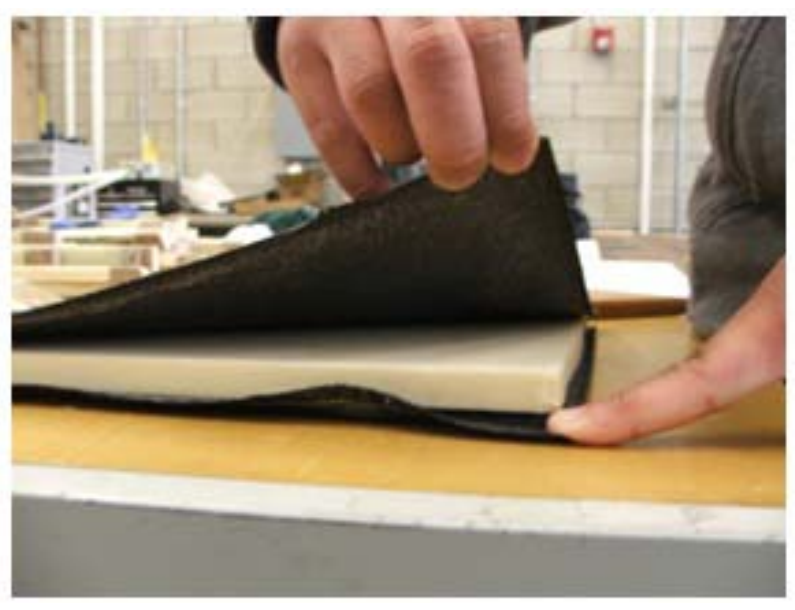

Figure 27: Delamination of face sheet from core for preliminary specimens

The specimens were redesigned. The width of the part was increased to 2”, while the length of the part remained the same from the new diameter of the hole. The addition of the film adhesive increased the thickness of the specimens by 0.020 ”. The new specimen's dimensions became 5” x 2" x 0.576"; this can be seen in Figure 28. The final specimen dimensions can be seen in Table 3. The hole locations for the new specimen dimensions would remain the same because the edge distance was still three times larger than the new hole diameter. The test results of the new specimens showed a better bearing failure compared to the previous specimen design. The new specimens had a more distinct bearing failure and were less inclined to a shearing failure. The new dimensions of the specimens were used as the final dimension for the experiment.

Table 3: Final Specimen Dimensions

\begin{tabular}{|l|c|}
\hline Bolt Diameter & $0.25 "$ \\
\hline Bolt Location from edge & $1.5^{\prime \prime}$ \\
\hline Hole Diameter & $0.375^{\prime \prime}$ \\
\hline Thickness of foam & $0.05^{\prime \prime}$ \\
\hline Thickness of each face sheet & $0.026^{\prime \prime}$ \\
\hline Specimen Length & $5.0^{\prime \prime}$ \\
\hline Specimen Width & $1.5^{\prime \prime}$ \\
\hline Specimen Thickness & $0.552^{\prime \prime}$ \\
\hline
\end{tabular}


The use of the film adhesive might cause two problems with the specimen: a change in the thickness of the specimens and an effect on the resin content of the face sheets, making them oversaturated. Since the specimens were manufactured by a press, the thickness of the specimens would vary depending on how much of the film adhesive was pushed out of the panel. The thickness of the specimens varied from 0.571 ” to 0.580 ” showing that the variance of thickness was within an acceptable tolerance. This implies that the press can create a consistent part every time. The second problem was the addition of extra epoxy to the laminate from the addition of the film adhesive. An increase in matrix or epoxy to a laminate would make the composite more ductile and decrease the overall stiffness of the part. This was undesirable because a more brittle specimen would produce a better bearing failure. To see if the specimen was oversaturated, a burn test was conducted and the Volume fractions of the matrix and fiber were calculated from its results; this will be further discussed in 6.1 Mechanical Characteristics of Material/Specimen

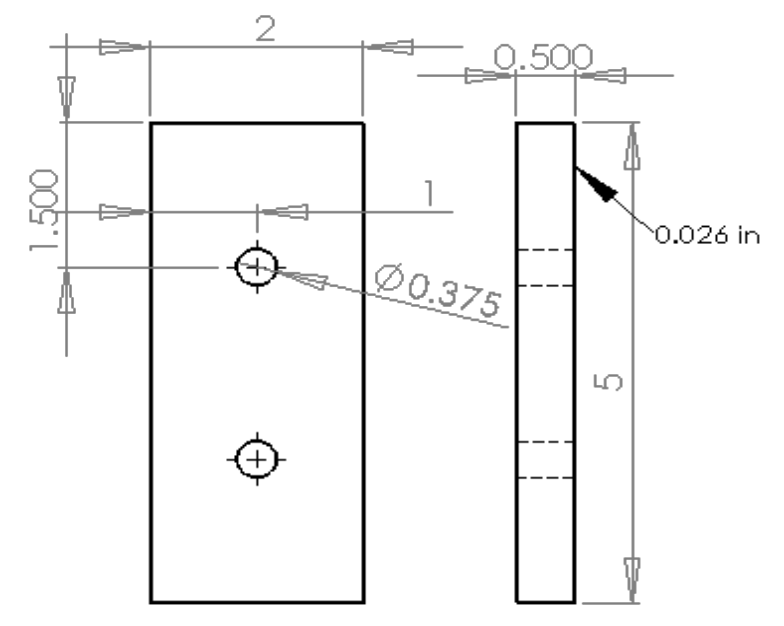

Figure 28: Final Dimensions of Specimens

\subsection{Damage Arrestment Device Design}

The preliminary damage arrestment device design was from Balatbat et al., where the devices were circular keys made out of unidirectional fiber glass and epoxy; this can be seen in Figure 29. The purpose of the damage arrestment device was to arrest the stress concentration 
from propagating along the part. This increased the structural strength of the composite panel by prolonging the overall failure of the panel. This type of damage arrestment device was used as the first generation of damage arrestment device.

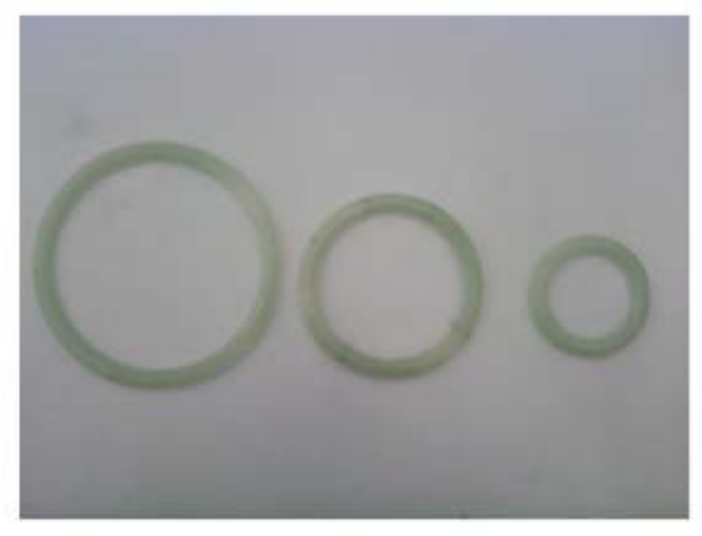

Figure 29: Circular Damage Arrestment Devices from Balatbat et al.

For the damage arrestment device production, a mold was manufactured ahead of time. A CNC mill was used to cut 4mm-deep 8mm-diameter semicircular grooves in a piece of metal. These grooves formed circles of diameters that varied from 2 in to 4 in. Aluminum was used for the mold because it reduced machining time.

After the mold was coated with Free-Coat or High-Temp Wax, the woven roving was pulled apart such that there was a large stack of large fibers remaining. For the manufacture of the device, twenty-eight large fibers were used for each damage arrestment device. This number was determined from trial and error and ensured that there were enough fibers to fill the mold but not too many as to prevent center fibers from absorbing the resin. Once the epoxy had fully cured, the damage arrestment devices were removed from the mold. The entire damage arrestment device came out as one piece due to the excess epoxy. They were then cut, separated, and sanded to remove any excess epoxy.

The foam was machined prior to inserting the devices. A manual mill was used for straight devices, but a CNC mill must be used in the case of circular devices. Each piece of foam was machined to the dimensions of the device used in each respective specimen. The devices 
were then fitted into the machined foam. The devices also needed to be sanded to ensure a tight, flush fit into the foam. Once the devices fit snugly in the foam, a small amount of epoxy was poured into the groove, and the devices were inserted. After these steps were taken, the new panel was laid-up using the VRI method.

Since the purpose of the damage arrestment device was to prevent a crack or failure propagating, the design needs to be augmented to be able to withstand forces acted from the fastener on the composite sandwich panel. The new damage arrestment device must be thicker and larger to accommodate the failure around the fastener. The first change to the damage arrestment device was to switch the composite material from fiber glass to carbon fiber, LTM45, by making it one homogenous material. The main factor for this decision was to make it easier for analysis purposes by making the composite sandwich panel as simple as possible. The use of LTM45 allows for a better bonding with the face sheet and decreases manufacturing time because the DAD could be cured at the same time with the face sheet instead of it curing separately. This will be talked about in 3.4 Damage Arrestment Device Prep.

The next change to the damage arrestment device was the geometry of the device. From the previous research, the devices were straight or circular devices with a semi-circle crosssection. The new geometry was a rectangular shaped design instead of a semi-circular device; this can be seen in Figure 30. The decision for using a rectangular device was to make it simpler for analysis of the interactions of the $\mathrm{DAD}$, face sheet, core, and fastener. A rectangular device makes it easier to manufacture the foam because it was easier to mill out straight slots instead of circular ones. A concern of changing the devices’ geometry was that a rectangular device would create stress concentrations on the corners of the device where it's bonded with the core, while a circular device would have an evenly distributed stress distribution on the core. Since this was to prove the concept of damage arrestment devices on prolonging failure along fastener/panel, the simpler geometry was used. 


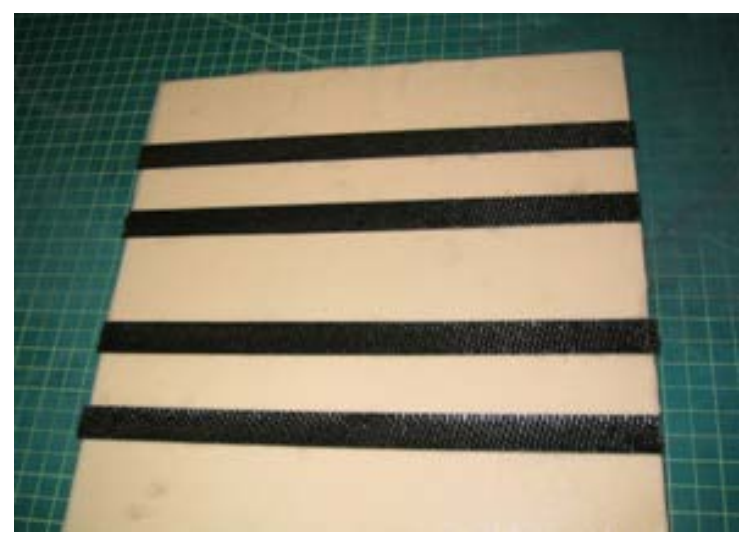

Figure 30: Damage Arrestment Device

The DADs went horizontally along the panel located along each hole on the specimen; this can be seen in Figure 31. The DADs had a width of 0.75, twice the diameter of the hole. Since this was a new concept, the effect of the width of the device was ignored. The thickness of the devices was dependent on the number of layers of LTM45, which has a thickness of 0.013” per sheet. The number of layers was varied until a sufficient trend was observed or until the layers dominate the thickness of the panel instead of the core. The dimensions of the DADs can be seen in Table 4.

Table 4: DAD Dimensions

\begin{tabular}{|l|c|}
\hline DAD Width & $0.75^{\prime \prime}$ \\
\hline DAD Location & $1.5^{\prime \prime}$ \\
\hline LTM Thickness & $0.013^{\prime \prime}$ \\
\hline DAD Thickness & Dependent on Testing \\
\hline
\end{tabular}

The use of LTM45 for the device allowed the specimens to be manufactured in two ways: cured separately or cured with the face sheet. Curing the DADs separately doubled the manufacturing time for the specimens because a laminate must first be manufactured and prepped and inserted for the curing of the face sheets. This was also highly undesirable because it created more stress concentrations on the foam since it was already cured when inserted. The devices underwent another cure cycle which altered the curing of the face sheet because the cured DADs act like a heat sink for the face sheets. Curing the DADs with the face sheet was advantageous 
because it had a better bond surface between the two and also had a lesser manufacturing time. Nonetheless, both manufacturing methods were investigated to see the difference between the two methods; this was further discussed in 6.3 DAD Manufacturing Test Results. The data showed that curing the DADs separately increased the strength of the specimen by $16.2 \%$, while DADs cured with the face sheet had a lower standard deviation. The method of curing the DADs with the face sheet was used for the manufacturing of the DADs because data accuracy was more important than an increase in strength.

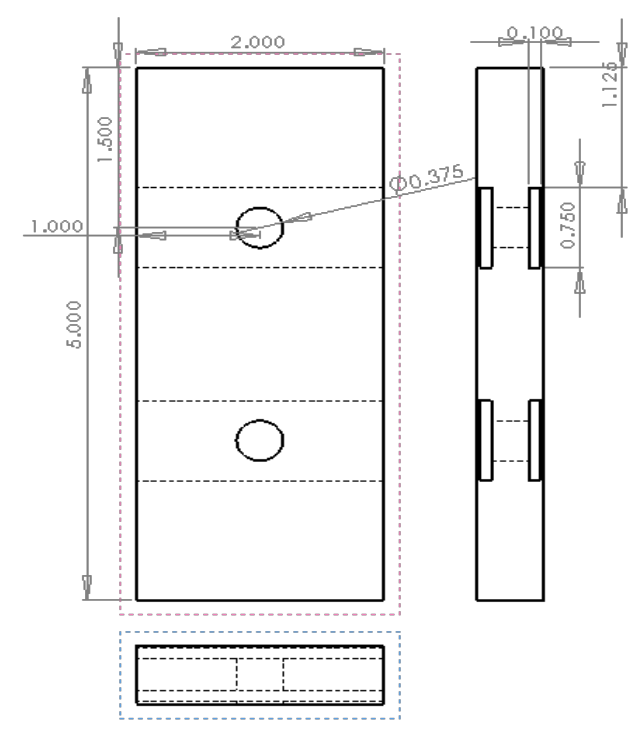

Figure 31: DAD Specimen Design

\subsection{Testing Apparatus Design}

The first generation of the testing jig design was based on the previous research that was conducted. Since the Aerospace Engineering Structures/Composites Lab contains an Instron 2743 Wedge Grips, the first testing jig was limited to the maximum width the jig could fit in the grips. With a geometry limitation, a 2D “anvil” jig was designed and created. The jig was based on the same testing apparatus from Demelio et al. and Song et al. Their apparatus, two metal plates attached to grips connected to the testing machines with a bolt attaching the composite panel to each metal plate. For the first generation test jigs, the apparatus would consist of two steel plates 
cut in the shape of an anvil. The handle part of the jig was 3” in length, 1” in width and a metal thickness of 0.5 ”. The hammer or anvil part of the jig had a length of 2", a width of 1.5” and a thickness of 0.5 ” The hammer part of the jig was supposed to have the same width as the specimens. A hole was located 0.5 ” from the edge of the anvil and centered along the width. The first generation test jig can be seen in Figure 32.

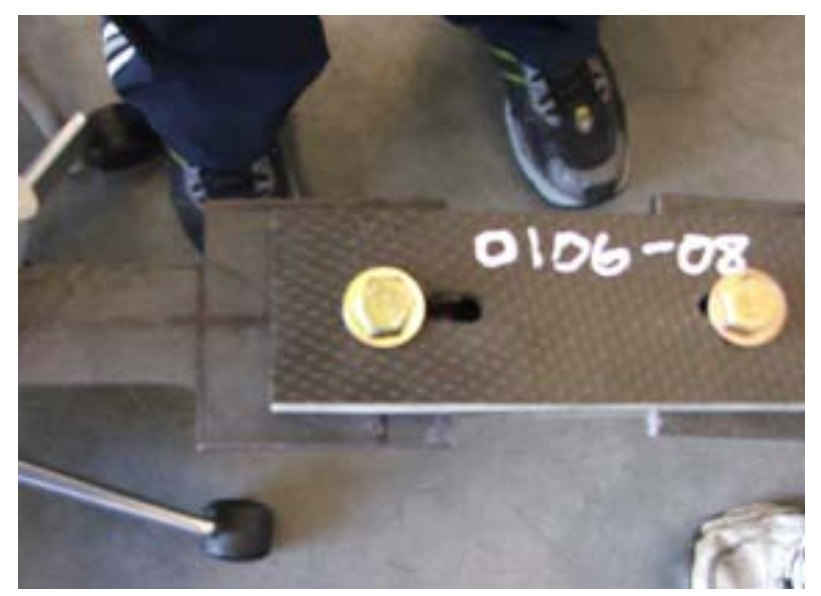

Figure 32: "Anvil" Jig

From the literature review, the testing apparatus had only one composite sandwich specimen on one side during testing. The problem with this set-up was that the specimens would not see a complete axial loading. The testing apparatus created a bending moment on the specimens which would distort the data coming from the machine. To prevent a bending load from being created on the specimens, a panel was placed on each side of the jig and tested at the same time; this can be seen in Figure 33. The new testing set-up created a true axial loading on the specimens, but showed inconsistent results between the two specimens. It would have been harder to analyze the two specimens at the same time because the data outputted from the machine assumed the test specimens were one piece rather than two. It was immediately decided that the test jig had to be replaced with a new jig that could only do one piece at a time and apply axial loading on the specimens. 


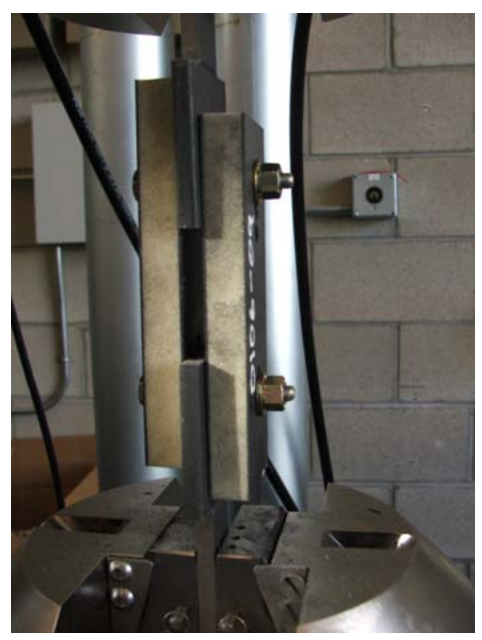

Figure 33: "Anvil" Jig with Multiple Specimens at the same time

The second generation jig was designed after the flaws from the previous jig were observed. The new testing apparatus consisted of a new three dimensional jig attached to the testing machine. The new three dimensional jigs consisted of an I-beam and a rectangular crosssection beam; this can be seen in Figure 34. An I-beam was cut in half to produce a T-beam that contained perfect perpendicular surfaces were attached to the Instron grips. A rectangular crosssection beam was cut in half to create a U shaped beam. The new U cross-section was cut down to the proper width of the specimens and a hole was drilled a quarter of the way up the length. The $\mathrm{T}$ and $\mathrm{U}$ sections were then welded together to create the new jig. The new jig was able to apply a pure axial loading on the specimens during testing. It was able to meet the requirements of the research under monotonic loading but when fatigue loading was applied on the jigs, a new problem arose. Under fatigue loading, the jigs became a tuning fork by vibrating in the horizontal plane. Due to being welded from two separate pieces, the jig was torque and bending at the welds. This gave erratic results in fatigue because the desired loads were not being applied properly to the test specimens. The jig was applying some shearing loads on the test specimens rather than the needed axial loading. A new jig must be designed to remove the horizontal movements that were observed during testing. 


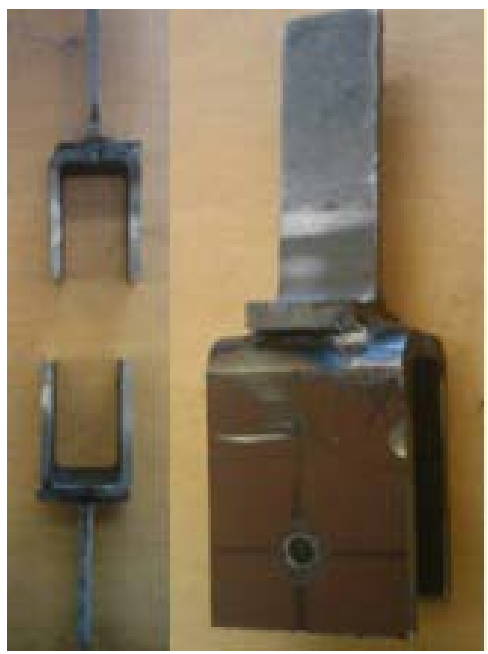

Figure 34: "U" Jig

To counteract the tuning fork tendency of the jigs under fatigue loading, a new jig needed to be designed from one piece to remove the welds. To do this the new jig must be milled from a block of steel. Since the jig was milled from a block of steel, it was also decided to make the new testing apparatus be a hybrid of a jig/grip; this can be seen in Figure 35. The new testing apparatus was attached directly to the machine, removing the wedge grips, and being the testing jig at the same time. This allows the jig to be in line with the machine, making it able to apply $100 \%$ axial force on the test specimens.

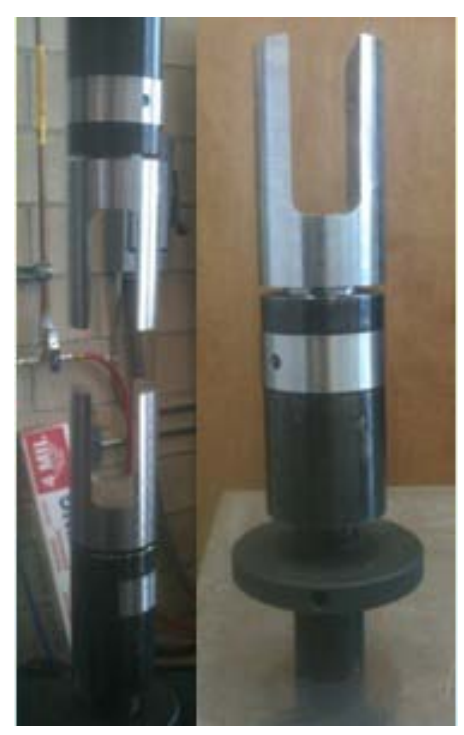

Figure 35: 3rd Generation Testing Jig (Final Jig Design) 
The new jig, or the $3^{\text {rd }}$ generation jigs, was milled out of a cylindrical stock of steel. The jig was manufactured by using a CNC lathe to face and turn the cylindrical stock into its grip and jig sections. Once the stock was faced and turned, the holes were drilled into the sides of the stock using the same machine. The test section part of the testing apparatus was machined out using a CNC end mill. To connect the testing apparatus to the Instron, a hole was drilled on the grip side so a steel dowel could be placed to attach it to the adapter that screws onto the machine; this can be seen in Figure 35 as the part of the testing apparatus painted in black. This process was repeated to create a set of two jigs for testing. The manufacturing of the $3^{\text {rd }}$ generation jig can be seen in Figure 36.
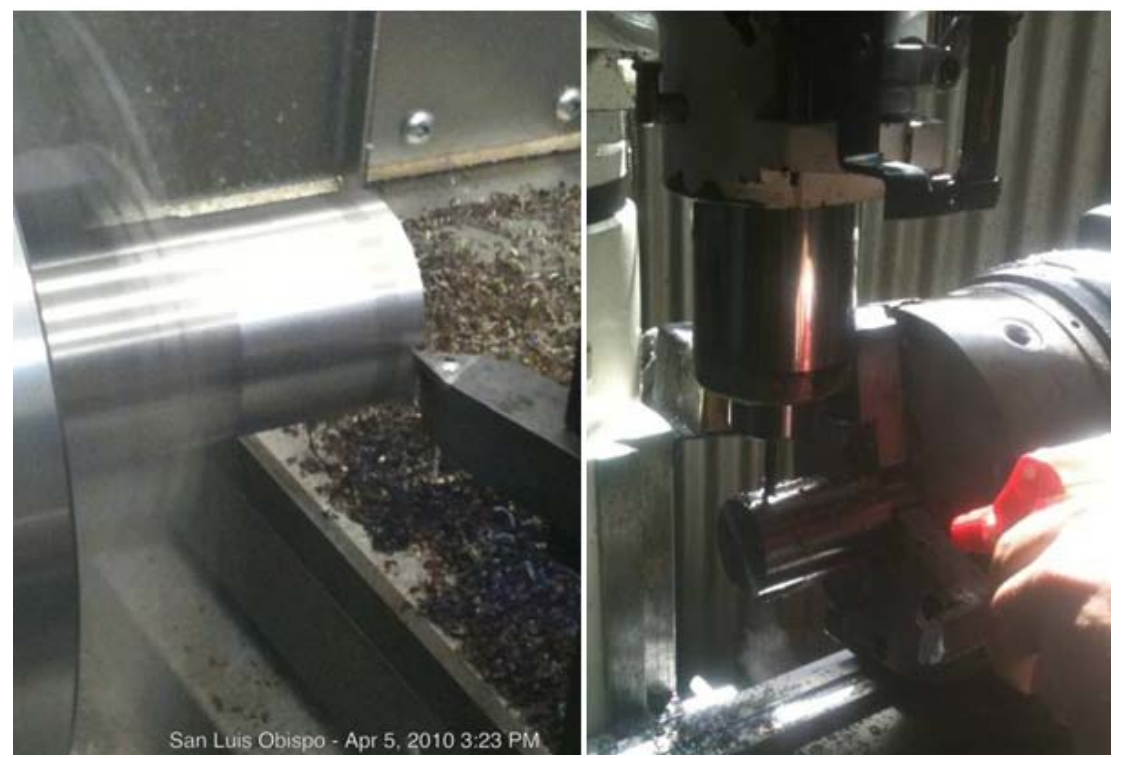

Figure 36: Manufacturing of 3rd generation Jig

\subsection{Hole Drilling Optimization}

The drilling of the holes in the composite sandwich panel was vital since the main objective was to study how the fastener affects it. It was discussed that the specimen needed a film adhesive to be added to the panel to prevent the delamination of the face sheet from the core. This was mainly caused by using a regular drill bit on the specimens. A drill press was used, in addition to drill bits, to drill holes in the composite sandwich panels. Drill bits contain flutes on 
them that push up removed material along the bit when in use. The mechanics of the bit caused an uneven pressure distribution between the core and face sheet which made the face sheet delaminate from the core; this can be seen in Figure 37. The face sheet easily came off the core due to lack of bonding from the prepreg carbon. Another problem with an ordinary drill bit was that burrs appear along the hole showing carbon being frayed from the rest of the laminate.

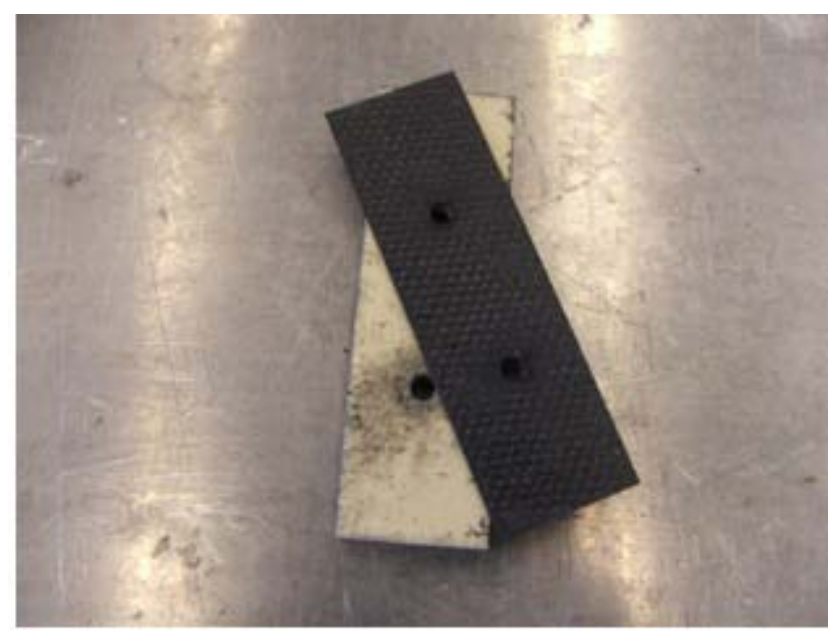

Figure 37: Face sheet delaminated from core

With burrs of carbon appearing around the hole from the use of an ordinary drill bit, other types of tools were investigated to drill holes into composite material. A tool that was looked at was a rotor tool such as a Dremal, instead of a drill press. Rotor tools have conical sandpaper bits that slowly step from a small diameter to the final diameter; this can be seen in Figure 38. The bit started from a 0.125 ” and gradually increased to 0.375 ”. This eliminated the fraying of the carbon by slowly removing material from the panel instead of at once from the drill bit. The tool was very effective in drilling the hole, but the bit created too big of a hole. Even though the final hole size was supposed to be 0.375 ”, the hole was a couple thousands of an inch too big, causing the bushing to easily slide out of the hole instead of being a snug or clearance fit. 

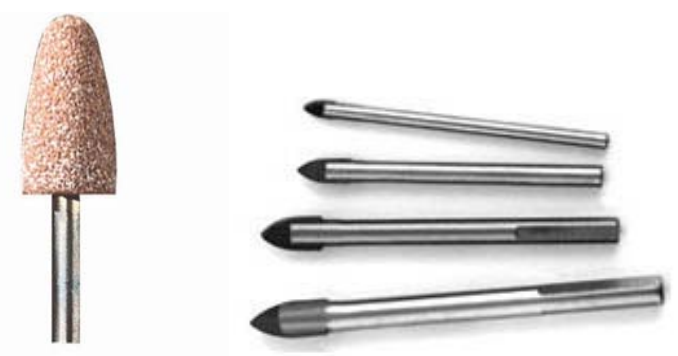

Figure 38: Bits used for hole drilling (Left: Conical Sanding Drill Bit/Right: Glass and Tile Drill Bit)

The next tool that was looked at was a glass and tile drill bit; this can be seen in Figure 38. The bit has a spade shape rather than a conical one. The bit, which was created by Black and Decker, was made out of steel and was tipped with a diamond dust coating to give a cleaner cut. The bit was designed to drill holes through tiles and glass which have similar brittle characteristics to carbon and other composites. It mimics the same drilling pattern as the conical sanding bit but gives a more accurate hole diameter. The hole was more of a clearance fit for the bushing and prevents it from easily sliding around during testing. A way to minimize the burrs more and to remove them completely will be discussed later in 3.5 Specimen Prep. The hole also had fewer burrs than the conical or ordinary bits. This bit was used for the drilling of the holes of the composite sandwich panels for the research.

\subsection{Summary of Design of Experiment}

The experiment consisted of a composite sandwich panel with dimensions of 5” x 2" x 0.552”. The composite sandwich panel consisted of a foam core, Last-A-Foam FR-6710, and a two layer face sheet of LTM45 on both sides of the core. Holes were drilled 1.5” from each edge on the lengthwise dimension with a hole diameter of 0.375 ”. The DADs were rectangular strips made of varying LTM45 thickness inserted around the holes. The strips had a width of 0.75 ”, twice the diameter of the holes. A bushing was used around the bolt to have an even pressure distribution along the composite panel. 
The experimental testing consisted of static and fatigue testing; the summary can be seen in Table 5. For static testing, the strain rate was tested to see the optimum rate to run the rest of the static testing. The DAD thicknesses were varied by the layers of LTM45 to see how it affected the interaction around the fastener/panel interaction. For fatigue testing, two test groups were tested with and without a DAD. The DADs had a 3 layer thickness for the testing. The fatigue amplitudes were varied from $65 \%$ of yield stress to $90 \%$ of yield stress. Each test case had 10 specimens.

Table 5: Cases to be tested

\begin{tabular}{|c|c|}
\hline Variables & \# Specimens \\
\hline 5 strain rates: $5 \mathrm{~mm} / \mathrm{min}-1.5 \mathrm{~mm} / \mathrm{min}$ & 70 \\
\hline 7 thicknesses: 1 layer -7 layers & $60-120$ \\
\hline 6 fatigue amplitudes: $65 \% \sigma_{\mathrm{y}}-90 \% \sigma_{\mathrm{y}}$ & $60-120$ \\
\hline 6 fatigue amplitudes: $65 \% \sigma_{\mathrm{y}}-90 \% \sigma_{\mathrm{y}} \mathrm{w} / \mathrm{DAD}$ & \\
\hline
\end{tabular}




\section{Manufacture Process and Prepping Procedure}

The detailed and step by step process of manufacturing and preparing the test specimen will be discussed in this chapter. The manufacturing of test specimens with and without DADs will be discussed from the cutting of the foam and composite materials to cutting down the panels to dimensions and the marking and naming of the test specimens. The manufacturing and testing process for the volume fraction and material properties of the test specimens will also be discussed.

\subsection{Foam Prep}

The foam was manufactured in sheets of 4' x 8' x 0.5 '”. The contact surface of the press was a 12” x 12”, meaning that the foam sheets had to be cut down to size to be able to fit within the surfaces of the plates. A table saw was used to cut the foam; this can be seen in Figure 39. The foam cutting needed three or more people to keep the foam steady as it was cut into 4' x 1' strips, allowing a total of 8 strips from each sheet. The strips were then cut to 12 ” x 12 ” plates. A total of 32 plates were cut from one sheet of foam. Safety was the biggest priority for prepping the foam because one wrong move could cause major injury from the table saw blade. Safety glasses and face mask were needed during the process to prevent the inhalation of foam particles during the prepping process. A total of about 37 plates were needed to meet the number of testing specimens.
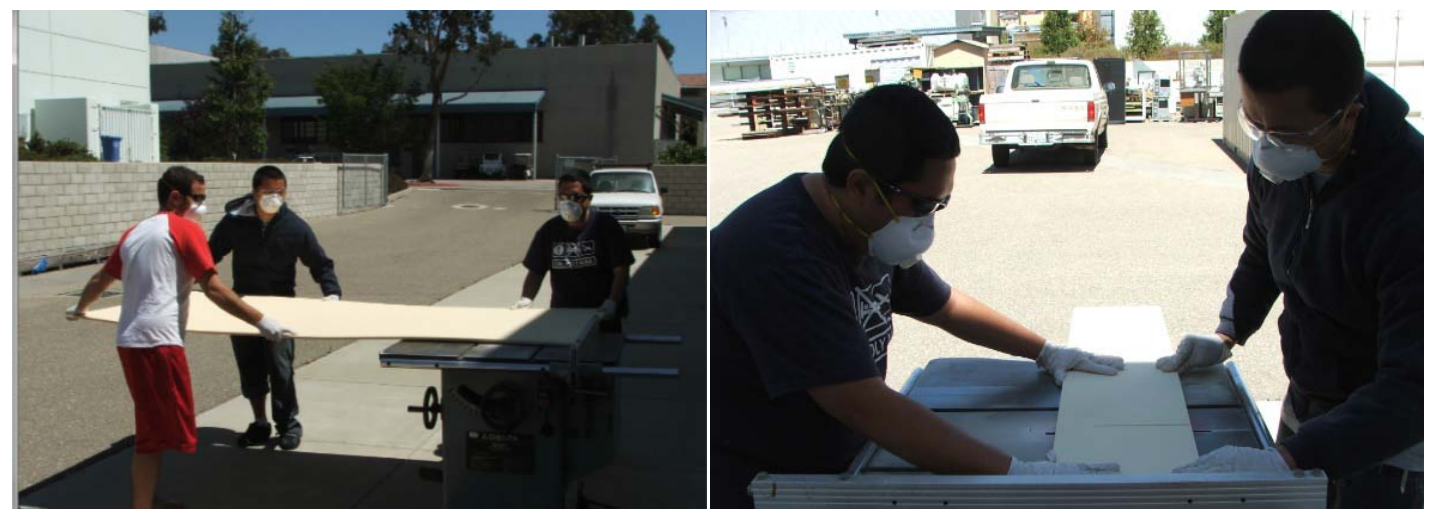

Figure 39: Cutting the Foam 


\subsection{Fiber Prep}

The face sheets and the film adhesive were cut into sheets. The LTM45 sheets were made into huge sheets that were rolled onto a tube and were stored in a freezer to keep the epoxy from curing. The amount of time the roll was removed from the freezer was recorded to make sure that the total time of the carbon did not exceed more than 24 hours. Having the roll out that long would cause a breakdown of the epoxy from the carbon and make it unsuitable for experimental testing. The rolls were removed from the freezer and set on a cutting sheet to be cut down to the exact dimensions. The sheet of foam used for that lay-up was used as a template to cut the LTM45 into size. A box cutter was used to cut the carbon; this can be seen in Figure 40. While cutting the LTM45, the carbon was cut between the fibers to prevent any fraying or unraveling of the carbon sheets. When the razor blade became dull, it was replaced to make sure frays are not created while cutting. Four sheets of 12" x 12” were cut and the roll was returned to the freezer for further freezing. The process for cutting of the face sheet was repeated for the film adhesive. The film adhesive was easier to cut than the carbon because fraying did not appear from the film adhesive.
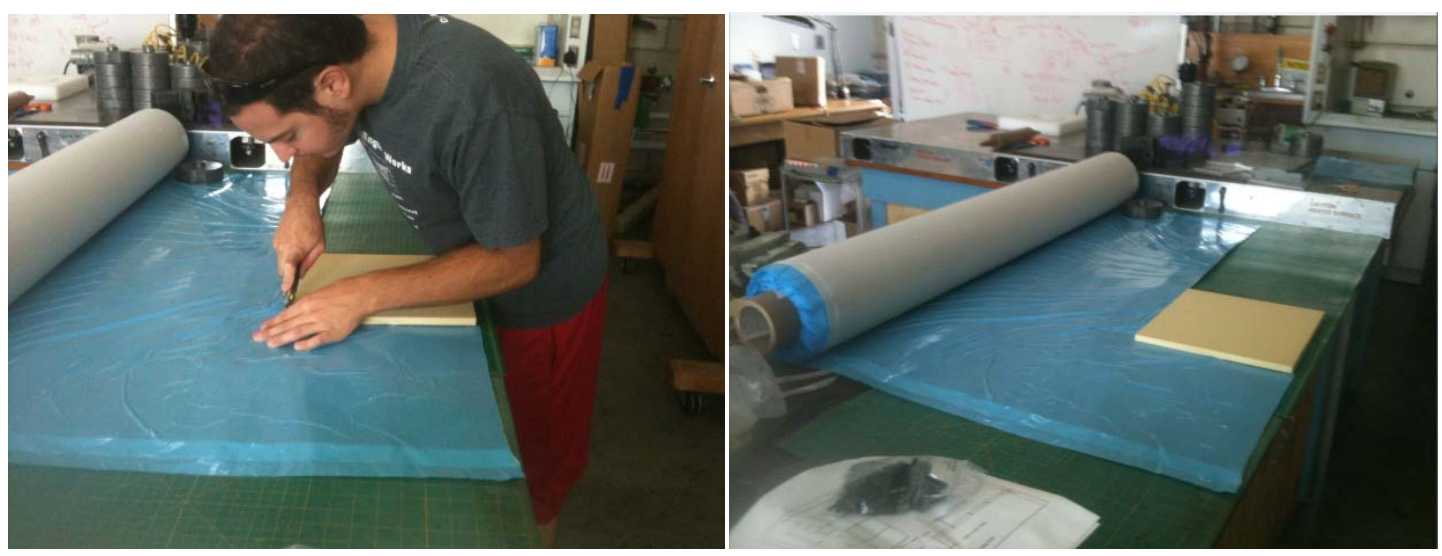

Figure 40: Cutting of the LTM45

\subsection{Composite Sandwich Panel Prep}

The composite sandwich panel consisted of the foam core, 4 sheets of LTM45 (2 layers on each side), and two sheets of film adhesive. The dimensions for all the materials were 12 ” $x$ 
12”. Two layers or two sheets of non-porous material were used between the composite sandwich panel and the metal plates for the press. The purpose of non-porous material for the lay-up was to absorb any excess resin from the plates and prevent it from curing on the plates and causing dimples on the face sheet. It acted like a protective barrier for the composite sandwich panels. The lay-up process for the composite sandwich panels can be seen in Figure 41.

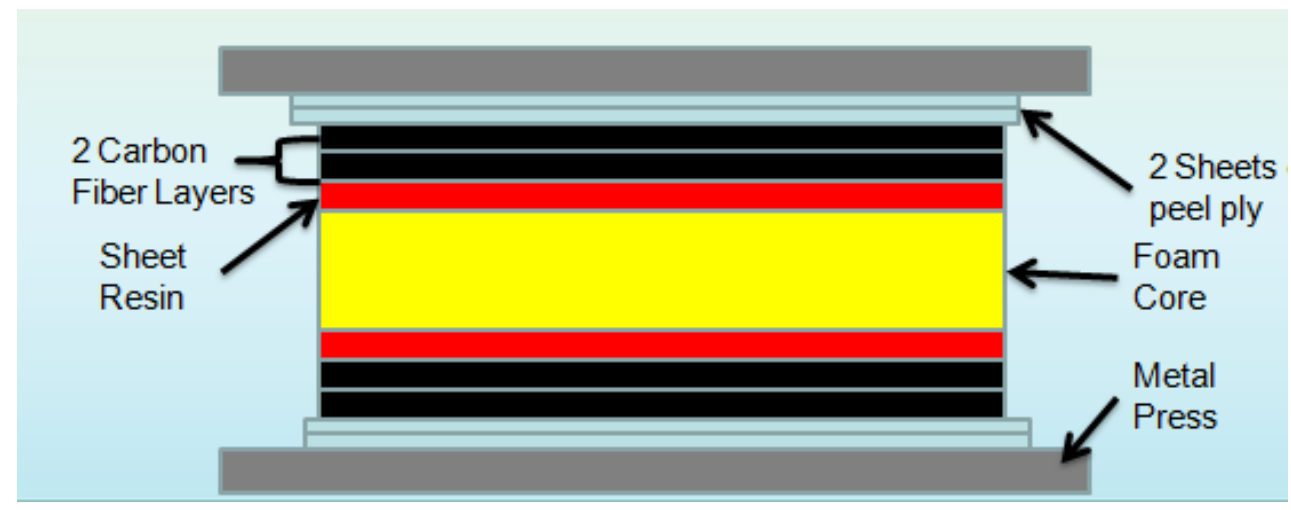

Figure 41: Schematic for Lay-up of Composite Sandwich Panels

The first step of manufacturing the composite sandwich panels was to sand and wax the press metal plates to ensure smoothness of the face sheets; this can be seen in Figure 42 . The plates were sanded down by using a high grit sandpaper to remove any resin that was cured on the metal plate. High grit sandpaper was used to prevent the removable of the magnetic coating on the plate. After the plate was sanded, a wax was used to smooth any divots on the metal plate. The wax was applied by hand and a buffer tool attached to a motorized drill was used to smooth out the wax that was on the plate. 


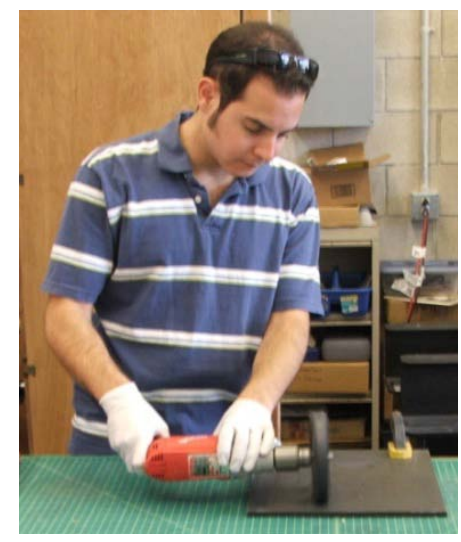

Figure 42: Sanding and Waxing of Press Plates

With all the materials prepped and ready, the materials were laid-up, as shown in Figure 41. The composite sandwich panel was placed into the press. The manufacture of LTM45 has a specific curing cycle, which cured the composite by applying heat and pressure on it for a specific amount of time. The manufacturer's curing cycle can be seen in Figure 43. The cycle first starts out with applying a constant load of 1000 lbs on the composite sandwich with the temperature increasing $2{ }^{0} \mathrm{~F}$ per min until the temperature reaches $150{ }^{\circ} \mathrm{F}$. The press then stays on for 16 hours at the temperature and the same force. After the time elapses, the temperature decreases $2{ }^{0} \mathrm{~F}$ per min until the temperature reaches $75{ }^{\circ} \mathrm{F}$ with a constant force of 1000 lbs. After the machine reaches room temperature the machine runs for another 2 hours with $1000 \mathrm{lbs}$ of force to end the cycle. According to the manufacturer, the composite was 98\% fully cured after the LTM45 has gone through the cure cycle. The plate would then be removed from the press and cut to dimensions of the test specimens, which will be further discussed in 3.5 Specimen Prep. 


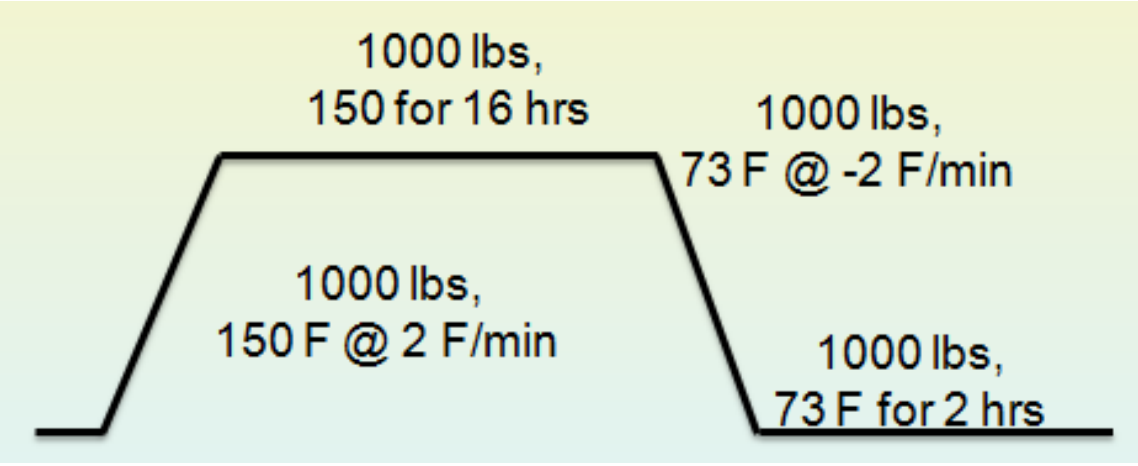

Figure 43: Curing Cycle for LTM45

\subsection{Damage Arrestment Device Prep}

Composite sandwich panels with DADs were manufactured first with milling the slots on the foam; this can be seen in Figure 44. A CNC end mill was used to mill the foam with a 0.75” four-flute straight end mill. A CAD model of the foam was made and was placed into CAMWORKS to create a CNC code to make the machine mill the foam to provide a better accuracy on the depth and alignment of the slots. Special care of the foam was vital for milling the slots to prevent the foam from being destroyed since it was flimsy. A vice grip cannot be used on the foam to keep it in place because it would leave mark and divots on the foam. To keep the foam in place, six toe clamps were used with three rulers going horizontally across the foam. The toe clamps and the rulers were placed around the slots to prevent the machine milling any of it out. The foam was milled on one side and flipped around to mill the other side, making sure that the milled out cuts was aligned on both sides of the foam. The mill depth of the foam was set to 0.039” which was the thickness of three layer of LTM. 


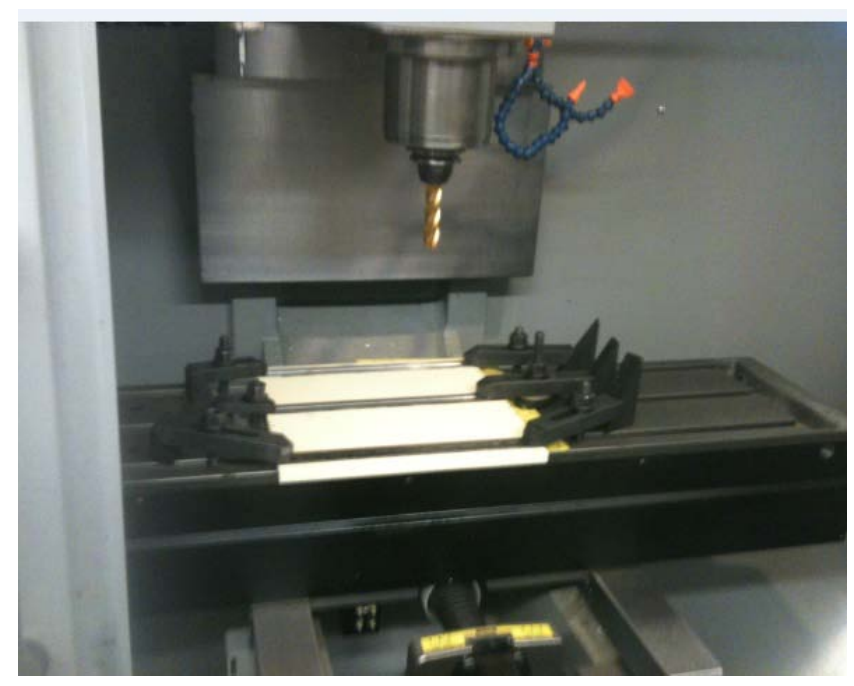

Figure 44: Milling of foam for DADs

With the foam milled, the DADs were created using LTM45 cut into strips of 12” $\mathrm{x}$ 0.75”. A 12” x 12” sheet of LTM45 was marked with dimensions of the DAD strips and was cut using a Rotatrim to have a clean cut; this can be seen in Figure 45. The marking of the sheet was done using a Sharpie and a ruler. This process was repeated until 24 strips are cut to for one 12 ” $\mathrm{x}$ 12” foam plate, with 3 layer DADs per slot.

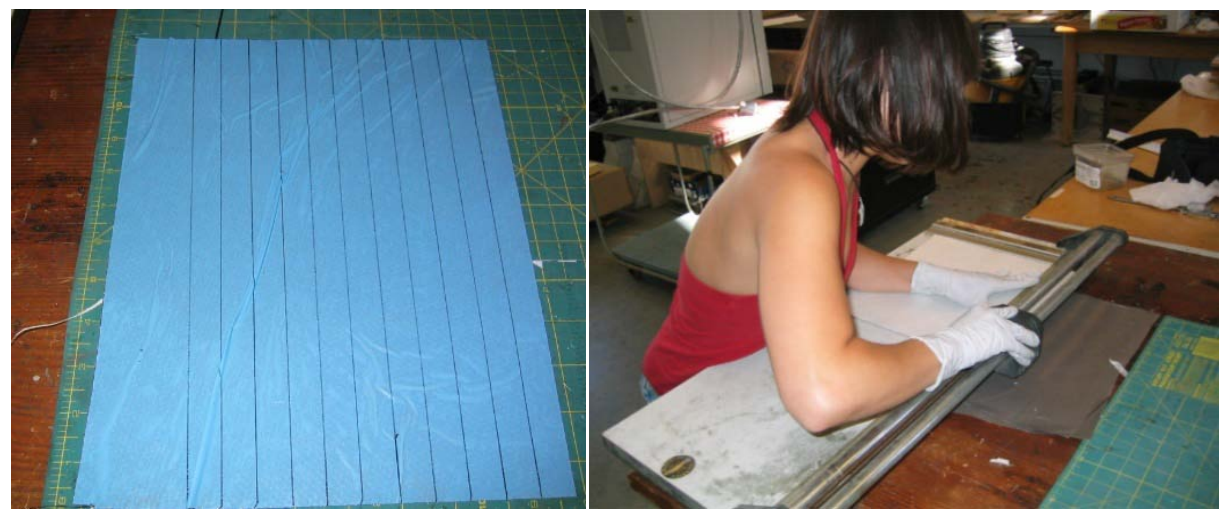

Figure 45: Cutting of the DAD strips

With the strips cut, the strips were set into eight piles of three strips. The protective papers of the strips were removed and laid into the milled out slots of the foam one at a time; this can be seen in Figure 46. 


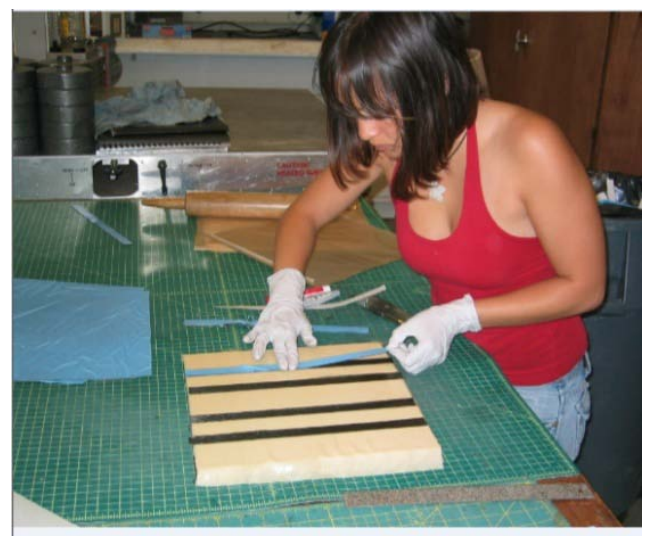

Figure 46: DADs being inserted into foam slots

The rest of the manufacturing of the plate was the same as the regular test specimens. It consisted of four sheets of LTM45 (two layers on each side), and two sheets of film adhesive, and 4 sheets of non-porous material (two sheets on each side). The panel went through the same curing cycle as before; the final product can be seen in Figure 47.

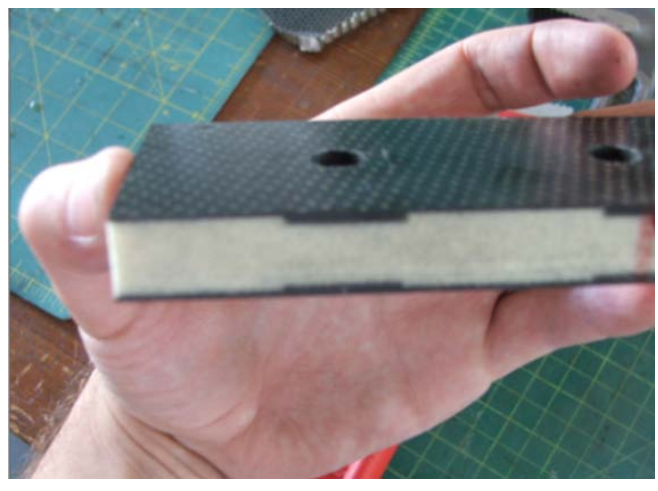

Figure 47: Finish specimen with DADs

\subsection{Specimen Prep}

The plates were then removed from the press and were prepped into the final specimen dimensions. The plates, without DADs in them, were first taken to the tile saw to be cut down into strips and then into test specimens. The first step was to trip down the edges of the plates to try to make the sides be perpendicular with one another. Since there was an inch of clearance on each side of the plate, up to a 0.75 ” could be removed from each edge. The next step in cutting with the tile saw was to use old test pieces to make sure the cut was the right dimension; this can 
be seen in Figure 48. With the right dimension in place on the tile saw, the pieces were cut into 2" width strips totaling five strips for each plate. The strips were then cut into two 5 ” length pieces. These pieces had the final specimen dimensions of 5” x 2” x 0.552 ”. A total of 10 test specimens were made out of each 12" x 12” plate.
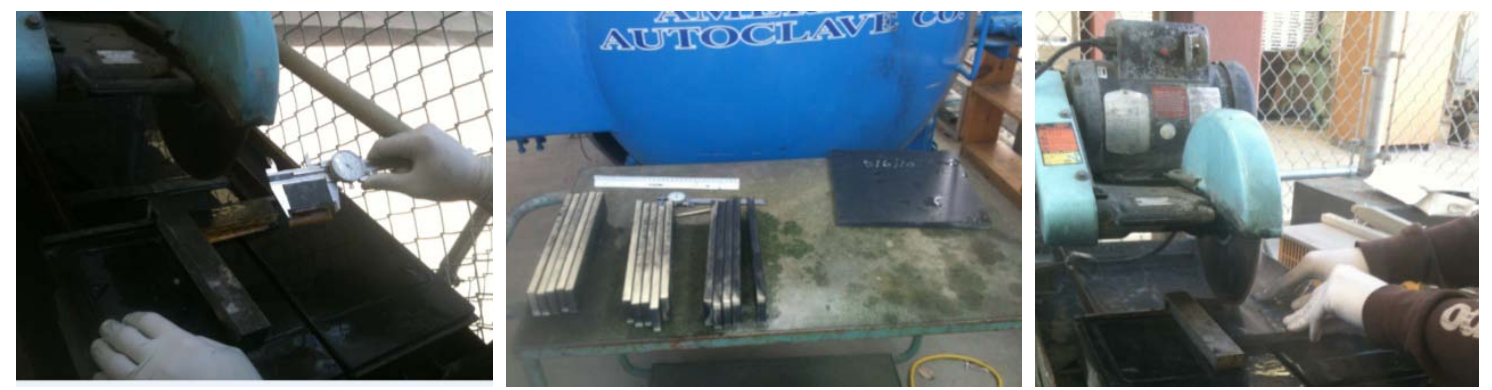

Figure 48: Cutting of Composite Sandwich Plates without DADs

For plates with DADs, special care was taken when cutting with the tile saw because it might cut improperly causing the DAD location from the edge to be shorter than the designed length. To prevent this, measurements had to be marked on the plate for it to be cut properly. The top most DAD location would measure and the half-way point of the DAD was marked on both side of the plate. A line then connected the two points. From the line, a new parallel line was marked 1.5” above it. This was called the main edge. The plate was then cut along the main edge. Next, the plate was cut into 2" width strips like before, making sure the DADs were perpendicular to the cut. The strip was then cut at five inch increments from the main edge to make two test specimens from each strip. This was possible because of how the slots of the foam were CNC and assumed to be accurate. This was repeated until 10 specimens were produced from each plate.

The test specimens were then drilled using the glass and tile drill bit on the drill press. The specimens, without DADS, were first marked by creating cross hairs on the face sheet using a caliber; this can be seen in Figure 49. The caliber was first used to measure the width of the specimen. That width was then divided in half and the caliber was set and locked at that new value. One side of the caliber was placed on the edge of the specimen and the other placed along the face sheet. The caliber was slowly slid across the face sheet creating a small line along the 
lengthwise of the specimen. This created the centerline for the width of the part. The next step was to set and lock the caliber to 1.5”. The marking from the caliber was then done on both edges of the face sheet. This created two crosses on the specimen with its centers being the points to drill the holes through.
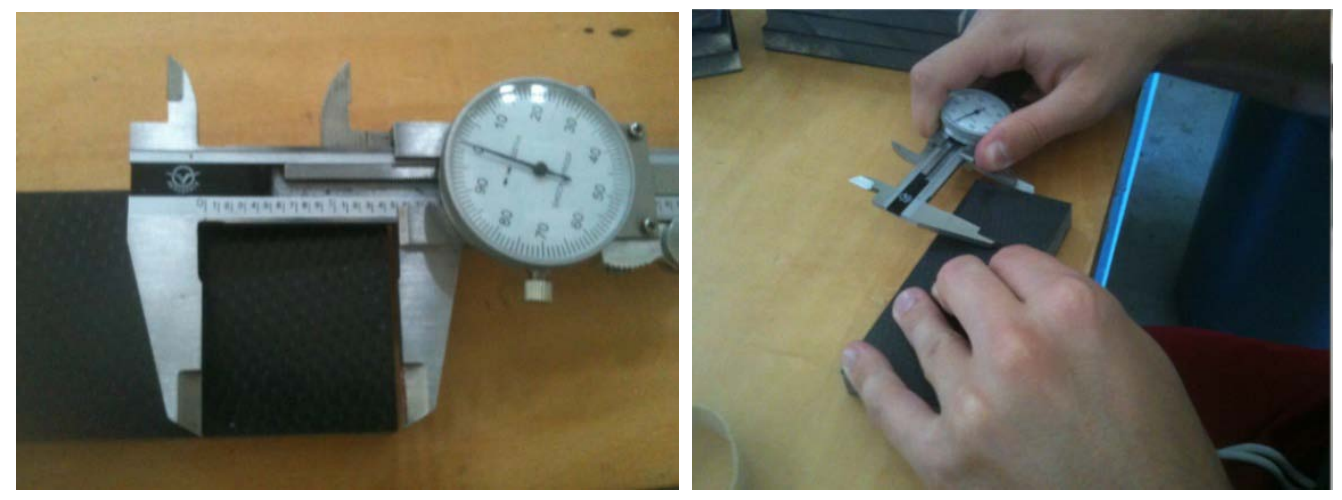

Figure 49: Marking the specimens for drilling

For specimens with DADs, a special tool was created to mark the midpoints of the DADs; this can be seen in Figure 50. The tool was created from a carbon laminate that was cut down to the length from edge to edge of both DADS on a specimen and a thickness of the composite sandwich plate. The tool had slots cut out of it representing the mid-point of each DAD on the specimen. Like previously stated, this was accurate because the slots were milled out with a CNC end mill. The tool was placed on both sides of the specimen and the mid-points were marked. A line was created from the two points with the help of a ruler. This would create the horizontal markings for the hole drilling. The vertical markings were done the same way as specimens without DADs. A caliber was used to mark the vertical line by measuring the width and dividing it in half. 


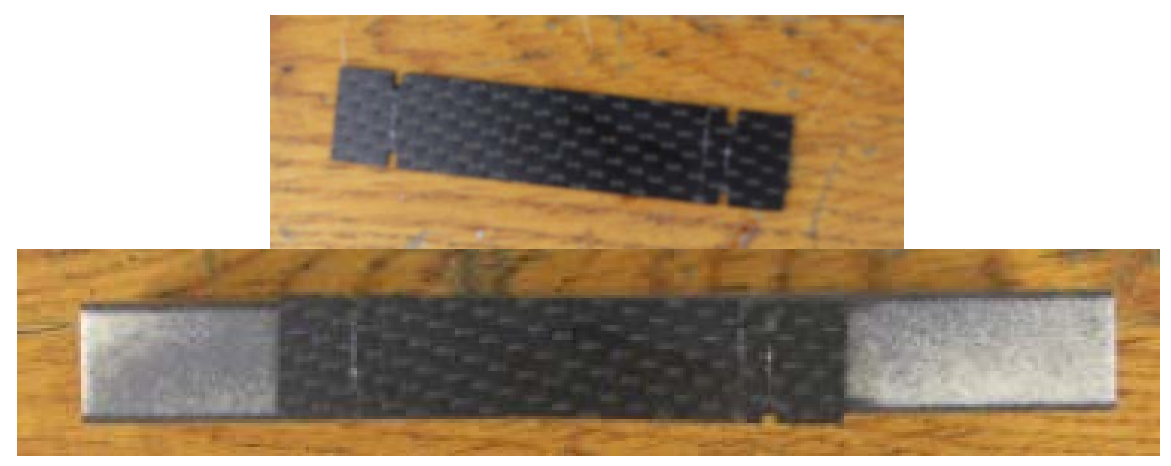

Figure 50: Marking specimens with DADS for drilling

The specimens were then drilled on the drill press. The drilling process was pretty simple, the vice grip was used to align the cross hair markings to the drill bit. Two pieces of cork board were placed at the sides of the specimens so the vice grips would not crush the specimen when it was tightened. Each hole was drilled and then deburred with a deburring tool; this can be seen in Figure 51.
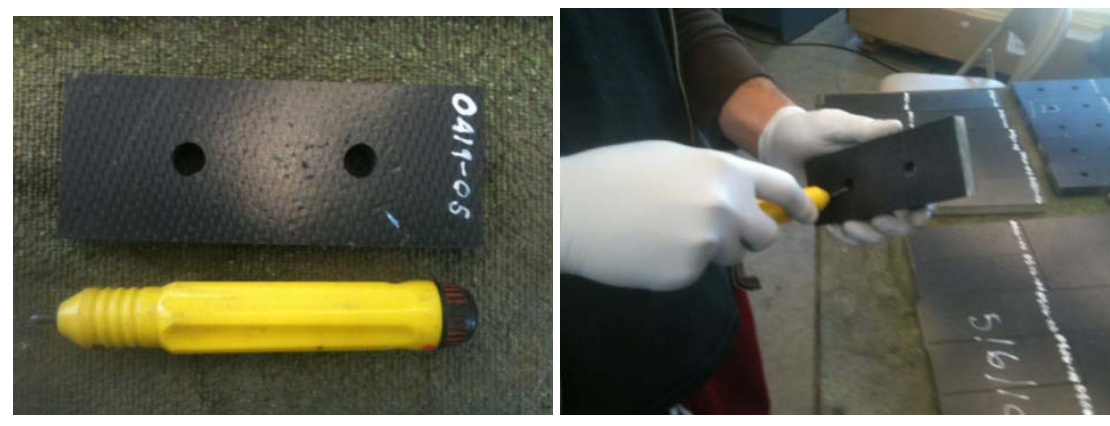

Figure 51: Deburring of holes

The specimens were then marked using a white out to know which specimen was which. A special naming system was used for the specimen; this can be seen in Figure 52. A six number naming system was used for the specimens, for example XXXX-XX. The first four numbers were the date that the plate was manufactured; for example, if a specimen was created on March 3, the first four digits were 0303. The last two numbers was the specimen number from that plate, having a maximum of 10 since only 10 specimens could be made from one plate. The specimens were then ready for testing. 


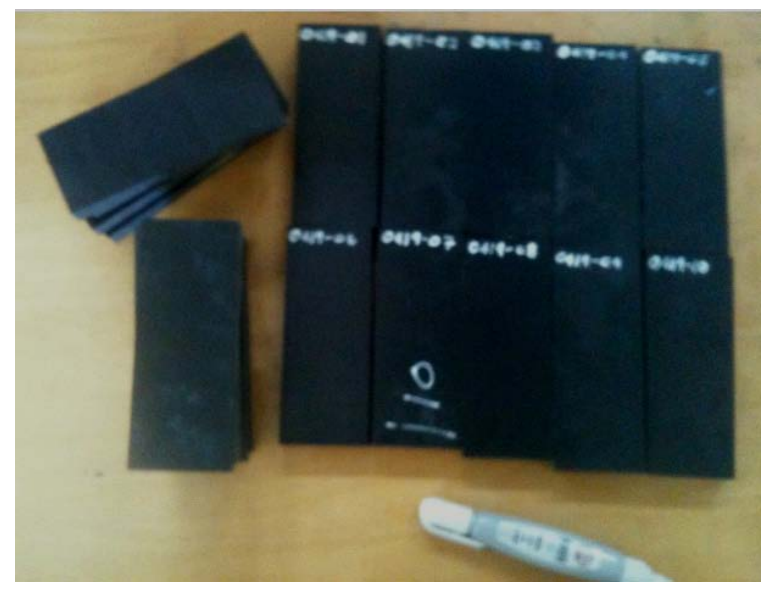

Figure 52: Naming of the specimens

\subsection{Testing Procedure of Volume Fraction of Face Sheet}

The volume fraction of face sheets was tested by obtaining a previous test specimen and removing the face sheet from the core. This was done on two test specimens with and without a film adhesive. The face sheets were first removed from the core by cutting the test specimen in half and sanding down as much of the foam as possible from the composite sandwich panel; this can be seen in Figure 53. A belt sander was used to remove excess foam to have as much as possible of the laminate for the volume fraction testing. This was done for both face sheets of the composite sandwich panel to see if the volume fraction was consistent on both sides of the specimen.
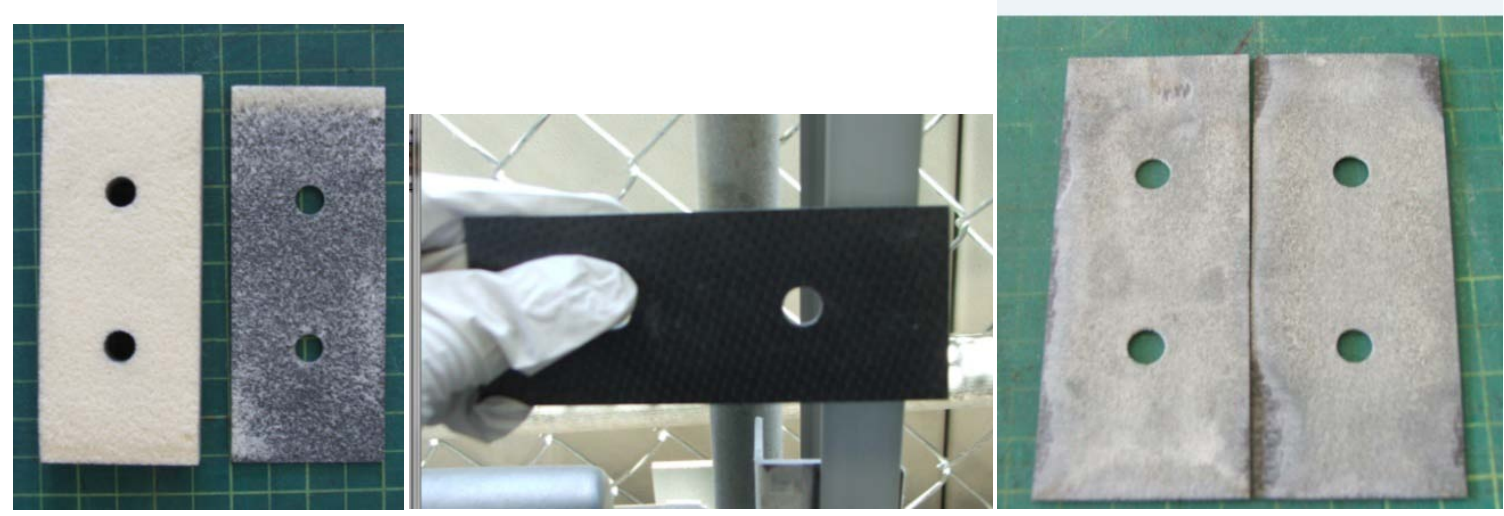

Figure 53: Removal of core from face sheet for burn test 
The specimens were then cut into six 1" x 1” specimens from each face sheet. The tile saw was used to cut the specimens. The specimen's dimensions were measured and recorded by a caliber. After they were measured, the specimens were weighed on a scale and the measurements were recorded. This all can be seen in Figure 54.
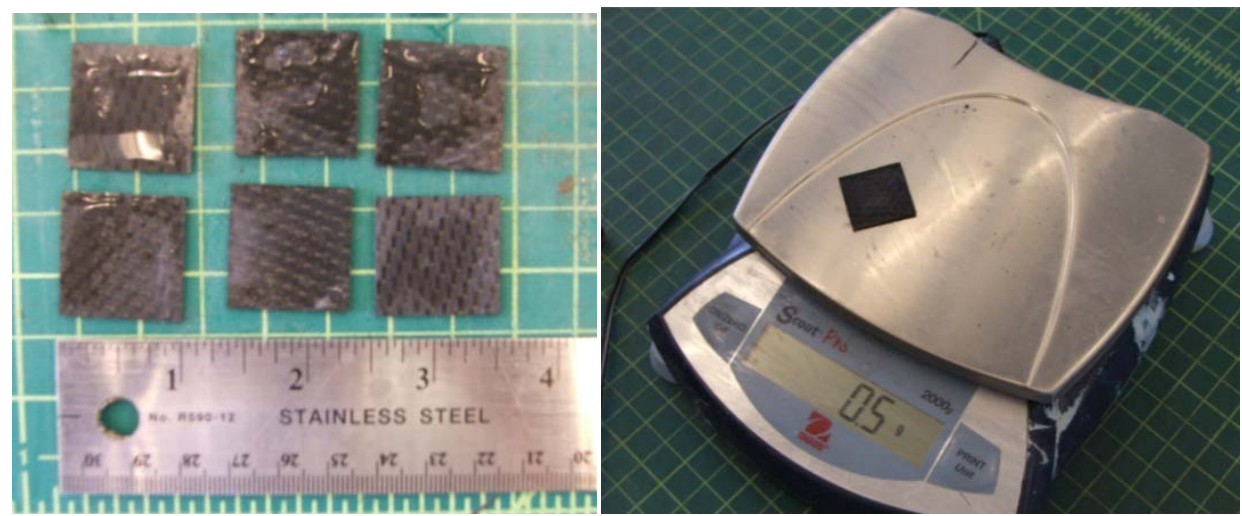

Figure 54: Measurements of specimens for volumetric fraction testing

The specimens were then placed into an oven and were burned at a temperature of 1000 ${ }^{0} \mathrm{~F}$; this can be seen in Figure 55. The specimens stayed inside the oven until all the epoxy was burned off the specimens, leaving only the carbon fibers. The dried fibers were weighed again with the scale to be used later to calculate the volume fraction. The specimens were stored and labeled later for further analysis.
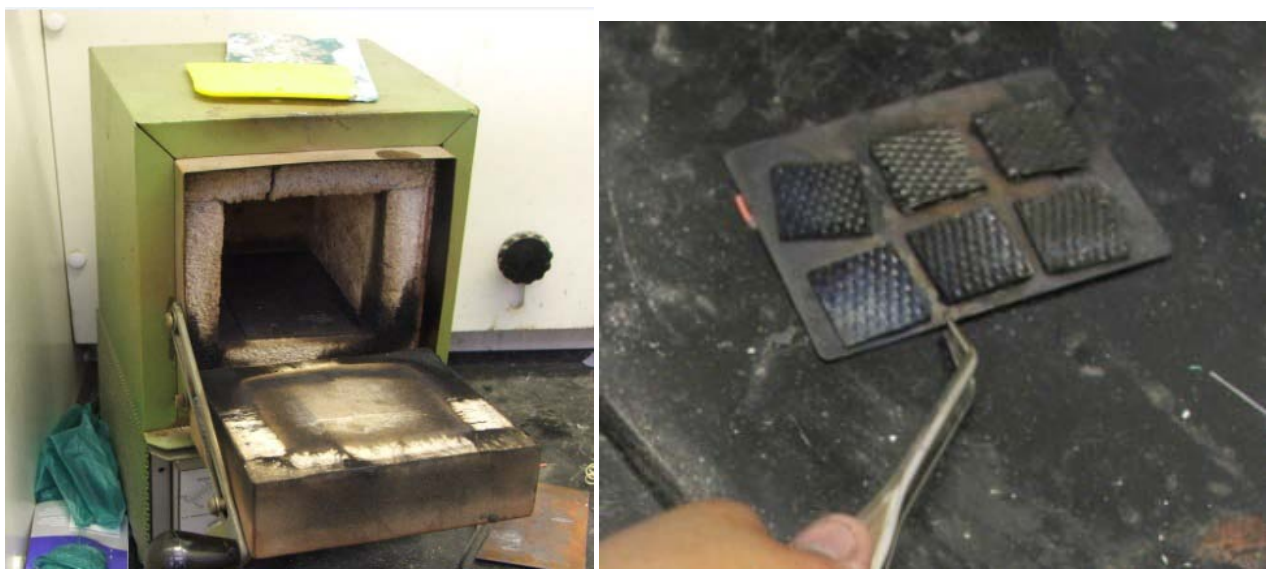

Figure 55: Volumetric Testing of specimens 


\subsection{Specimen Preparation for Material Properties Testing}

The overall elastic modulus of the composite sandwich panel needed to be tested without any holes in the panel. A notched or holed specimen would have a higher yield stress and a lower elastic modulus than the test specimens. These values were compared to the theoretical analysis for calculating the overall elastic modulus of the specimens. The specimens followed augmented testing procedure from ASTM C364 standard. The standard lay out was that the procedure must have a flat surface applied to the panel. Since the manufacturing process could not produce a straight cut, wood was added to the plate to provide an even distribution on the specimens; this can be seen in Figure 56. The wood was a proven testing procedure from previous research.

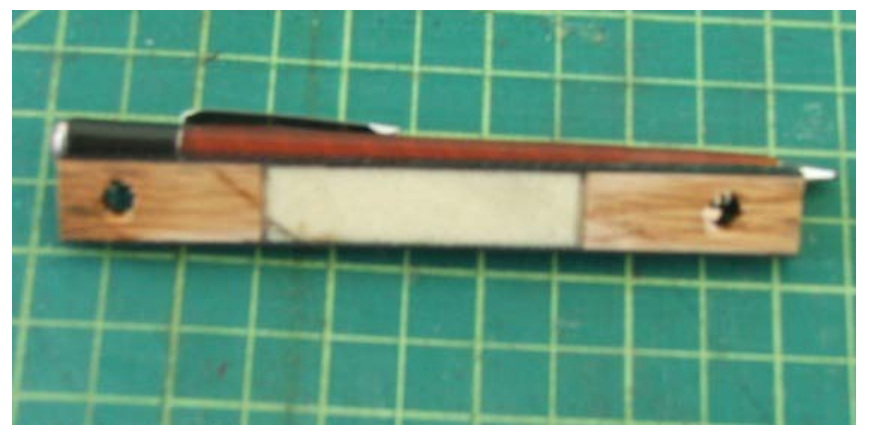

Figure 56: Elastic Modulus Testing Specimen

The specimens were first manufactured by cutting the foam down to dimensions of 2” $\mathrm{x}$ 10 ”. The wood had a dimension of 1.5 ” x 10 ”. Both had a thickness of 0.5 ”. The two materials made up the core for the composite sandwich panel; this can be seen in Figure 57. The foam was placed between the two pieces of wood. To keep the core in contact with one another, the film adhesive was placed on both sides of the core. Two sheets of LTM45 were placed on top of the film adhesive. The face sheet set up was similar to the regular test specimens. The specimens were placed in the plate and follow the same curing cycle with only one change. Since the test specimens were smaller in dimensions, the load on the elastic modulus testing needed the same pressure as before. The load applied to the specimens was $400 \mathrm{lbs}$ instead of $1000 \mathrm{lbs}$. 

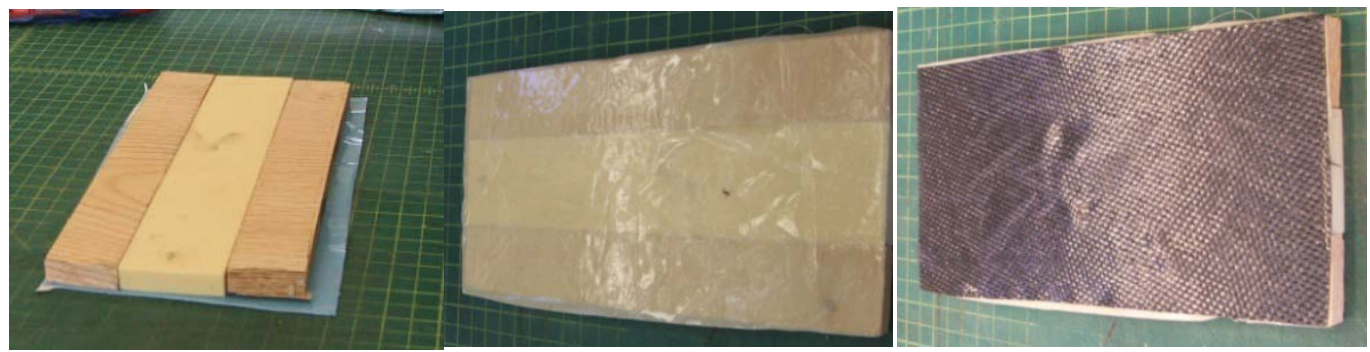

Figure 57: Manufacturing of Elastic Modulus Testing Specimens

Once the specimens were done manufacturing, the plate was cut down to one inch width specimens. The final dimensions of the test specimens were 5” x 1” x 0.552 ”. Holes were drilled through the thickness section of the test specimen instead through the face sheet. The hole was drilled through the wooden section of the core. The hole had a diameter of 0.25 ”. This method produced a constant loading on the wood which transferred an evenly distributed loading on the foam core and face sheet. The testing was conducted using static loading and similar to the regular testing procedure. 


\section{Experimental Set-Up}

The experimental set-up and testing of the research was then designed. The strain rate to run the speed of test of the Instron machine for all monotonic testing was investigated. The failure criterion for monotonic loading was also decided for consistency between the results. The design of the fatigue testing for the research was conducted, such as the failure criterion and the frequency of the machine. Lastly, the procedure for setting up and running the experimental test was discussed.

\subsection{Strain Rate Testing Selection}

A strain rate, according to testing standards, was important for monotonic testing of composites. Since each composite structure was greatly different from one another, one type of strain rate for one structure was bad for another. A quick strain rate distorted failure results by moving the failure of the fastener/panel interaction quickly from bearing to shear or even to a complete failure of the panel. A slow strain rate gave good results but would take too long to test each specimen. For the strain rate selection, 5 strain rates were tested, ranging from $0.5 \mathrm{~mm} / \mathrm{min}$ to $1.5 \mathrm{~mm} / \mathrm{min}$ with 10 specimens per test case and a variance of $0.25 \mathrm{~mm} / \mathrm{min}$ between the strain rates. The standard for testing metal pieces was a strain rate of $1 \mathrm{~mm} / \mathrm{min}$. Since there was no set standard for strain rate for composites, the speeds that were chosen are reasonable to test two rates above and below for the composite sandwich panel. A failure criterion of a 30\% load drop was chosen as the end of test for the monotonic testing. This meant that a total failure drop of $30 \%$ for the most maximum load would stop the machine.

All specimens were tested and analyzed; this was discussed later in detail in 6.2 Strain Rate/Control Group Results and will be briefly discussed in this section to choose a strain rate for all static testing. A summary of the results was shown in Table 12 in 6.2 Strain Rate/Control Group Results. The results showed that all five strain rates had similar failure load varying from $890 \mathrm{lbs}$ to 1,011 lbs and had similar elastic modulus varying from 45,170 psi to 50,250 psi. The 
results showed that the strain rate did not greatly affect the characteristics of the panel, but it did show a variance in standard deviation for each case. The table shows that the slowest rate, 0.5 $\mathrm{mm} / \mathrm{min}$ had the lowest standard deviation for both failure load and elastic modulus. This rate was used for all static testing because it gave the best standard deviations compared to the other rates translating a better accuracy to future tests.

\subsection{Static Testing Design}

From the detailed literature review, all of the fastener/panel interaction testing was conducted under a tension loading. A compression loading was chosen because a compressive loading would produce a more important failure mode to the panel due to buckling being seen during the testing. A buckling failure was more of a catastrophic failure for a panel than a tear or crack propagating from a tension load. The static testing portion of the experiment consisted of finding the optimum strain rate for testing pieces under monotonic loading and finding the trend of how increasing the thickness of the DADs affect the fastener/composite panel interaction.

From the previous section it was decided that for all static testing the strain rate for the machine to run test was $0.5 \mathrm{~mm} / \mathrm{min}$ or $0.0197 \mathrm{in} / \mathrm{min}$. The DAD thickness was varied from 0 layers to 7 layers. Each case had a total of 10 specimens to observe the interaction regardless of whatever the standard deviation for the case was. A small standard deviation was not necessary since a proof of concept was the purpose of the research and it was assumed that it would have an adequate standard deviation from the results shown in the previous section. A failure criterion of a 30\% load drop was chosen as the end of test for the monotonic testing. This meant that a total failure drop of $30 \%$ for the most maximum load would stop the machine.

\subsection{Fatigue Testing Design}

Fatigue testing was when a part was loaded to certain value, usually below the yield stress of the material, and was applied a load amplitude to be cycled. This would show when the material or case would fail over time from normal loading. This was helpful with designers 
because knowing how the material fails helps design how and when the overall system fails. The load amplitude was usually a sinusoidal wave with its load value varying a percent difference from the yield stress; this can be seen in Figure 58. The test was first ramped to half the yield stress. From the detailed literature review and ASTM standards, the yield stress was defined as where the first drop on the stress-strain curves; this was seen in Figure 59. The stress was converted to load by the geometry of each test piece, which became the force yield. The amplitude, or A, was a dependent on the fatigue testing and what the researcher was investigating. One fatigue cycle was equivalent to one cycle of the sinusoidal wave. The frequency of the wave was arbitrarily chosen by the researcher. Once the test reached its failure criterion, the test stopped and ramped the load back to zero. The criterion was dependent on the researcher. There are two ways to define the failure criterion, a drastic load drop or a drastic change in position of the test specimen.

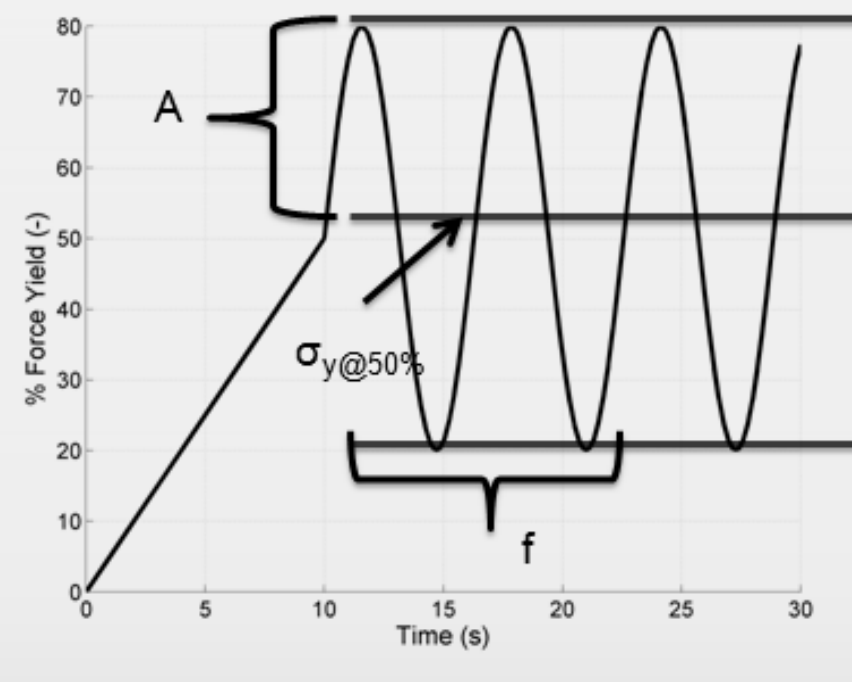

Figure 58: Fatigue Cycle 


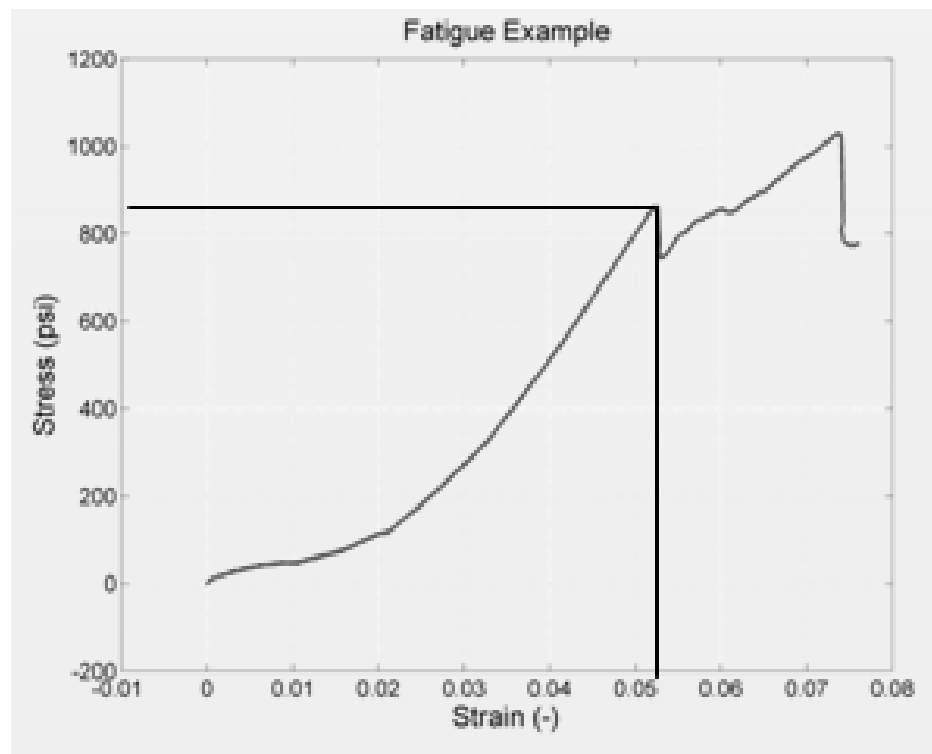

Figure 59: Yield Stress and Failure Criteria for Fatigue Testing

Two selections were made to fatigue testing for the experiment. The first selection was to choose the failure criterion for the testing. Since the yield stress was chosen as the maximum for the amplitude, the strain at that stress on the stress-strain curve was chosen as the failure criterion. The strain, like the stress, was converted to extension based on each test specimen's geometry and was used as the position failure for the test. Once the test specimen was moved by the amount of the extension, the specimen was defined to reach failure. This was also supported from preliminary testing by having a bearing failure around the holes; this was further discussed in 7.2 Fatigue Loading Failures.

The second selection for fatigue testing was to choose the frequency to run the testing. A too high frequency gave inaccurate results because it applied too many cycles per second on the specimen. A low frequency gave more accurate results but took too long per specimen to run. Two frequencies were investigated to see which one to use for fatigue testing, 5 and $10 \mathrm{~Hz}$. Both test cases were tested with 10 specimens each. The numbers of cycles of failure and its standard deviation for the two frequencies are shown in Table 6 . The results show a drastic difference between the two frequencies, the lower frequency had a lower number of failure cycles while the 
higher frequency had a higher number of failure cycles. For standard deviation, both had the same similar trend. Since both had high standard deviations, the higher frequency was chosen because it seemed that it would produce a higher average number of cycle failure. It also gave a decent run time, especially if the specimens reach the hundreds of thousands of cycles, which would mean hours to run one specimen.

Table 6: Summary of Fatigue Frequency Testing Results

\begin{tabular}{|c|c|c|}
\hline Frequency & Avg \# of Cycles & Std Dev \\
\hline$(\mathrm{Hz})$ & $(-)$ & $(-)$ \\
\hline 5 & 5555 & $69.46 \%$ \\
\hline 10 & 7032 & $87.41 \%$ \\
\hline
\end{tabular}

For the fatigue testing for the research, two case groups were run to compare the effects of the DADs on the composite sandwich specimens. The first group was the control group, no DADs in the specimens, and the other group had only one type of DAD thickness in each specimen. The trend of how the number of failures increases as the fatigue amplitude decreased was observed and analyzed for both case groups. The trends showed how the DADs affect the overall lifespan of a composite sandwich panel and how the failure around the fastener happened. It was assumed that different DAD thicknesses would have similar trends but was shifted along the graph. The amplitude for each case group was varied from $90 \%$ yield stress to $65 \%$ yield stress with $5 \%$ spacing, with a total of six test cases. Each test case had a total of 10 pieces.

The thickness of the DADs for the other case group was 3 layers. This was chosen because too thick of a DAD would affect the core/face sheet interaction. The ratio of the total thickness of the DAD over the thickness of the core was set to less than 0.2 . The ratio value assumed that the insert thickness was still within the infinite range, similar to the ratio value for the hole diameter and the width of the specimen. The 3 layer DADs was the closest to the ration without passing the value, making it the best choice. 


\subsection{Static/Fatigue Testing Set-up}

The static/fatigue testing of the specimens was pretty simple and straight forward. The specimens were installed on the jig and the software for each respective testing was conducted. The testing specimens were first measured and recorded. The width, length, and thickness of the specimens were measured using a calculator. The measurements were recorded on Goggle Docs for easy access anywhere; an example of the recording of measurements can be seen in Figure 60 .

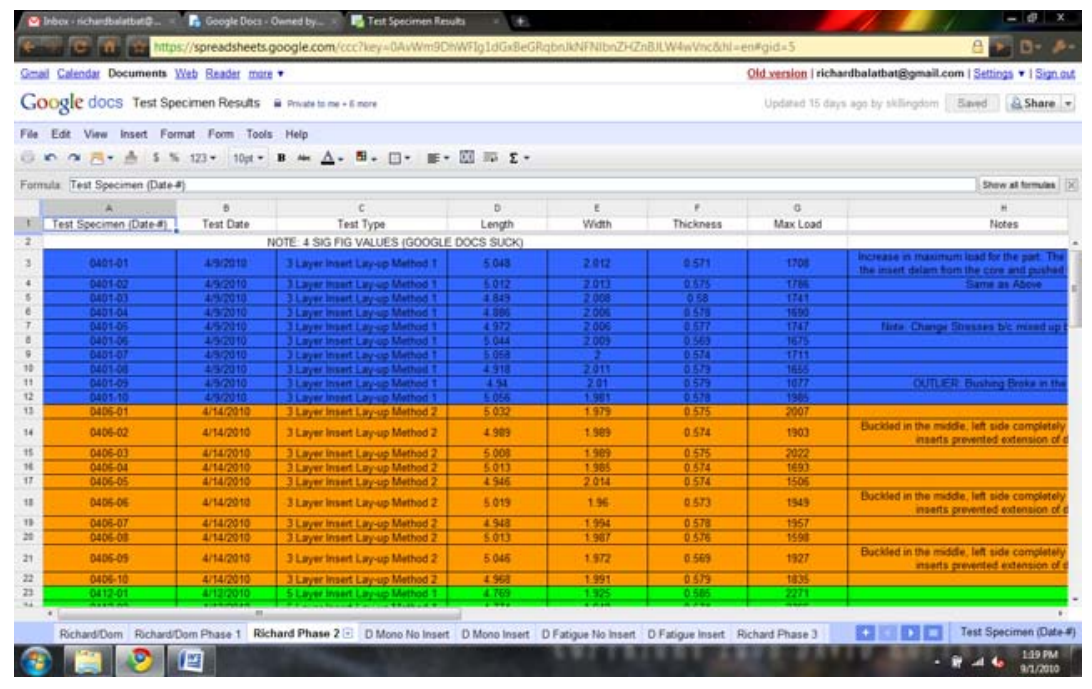

Figure 60: Example of recording measurements in Goggle Docs

Specimens were then taken to the machine and attached to the jig; this can be seen in Figure 61. Bushings were placed inside the hole and lined up with the holes on the jig. The bolts contained two washers, placed on the outside of the jigs so the bolt and nut heads were not touching the jigs. The bolt was tightened until resistance was made with the jig. The software was then being run until the specimens reach the failure criteria. For static testing, the failure criterion was when the load drops more than 30\%. For fatigue testing, the failure criterion was when the position of the test specimens moves a certain amount. For specimens without DADs, the failure criterion was 0.0544 ” and for specimens with DADs, the failure criterion was 0.0597 ”. All values were calculated from experimental testing. Once the specimens reached the failure criterion, the specimens are removed from the testing apparatus and pictures were taken to document the 
failure for each specimen. The load failure was recorded on Goggle Docs for future analyses. The specimens are stored for later use and analyses.

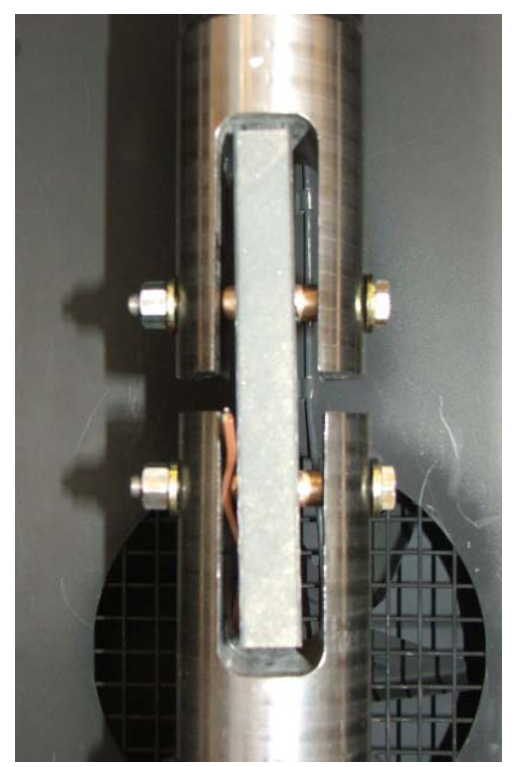

Figure 61: Testing Set-up 


\section{Theoretical Analyses}

The theoretical analyses of the composite sandwich panel will be talked about in this chapter. The analyses of the volume fraction of the composite face sheets were determined to investigate the ratio between the fiber and the matrix of the composite laminate. The theoretical Elastic Modulus of the composite sandwich panel was also analyzed and investigated using the extensional stiffness matrix of isotropic composite laminates.

\subsection{Analysis of Volume Fraction of Face Sheets}

An important factor to determine the properties of composites was the relative proportions of the matrix and reinforcing material. The properties can be obtained from two types of fractions, the weight fraction and the volume fraction. The weight fraction was easier to obtain during fabrication or by experimental testing. The volume fraction was the harder of the two to obtain, but was used more in the theoretical analyses of composite materials. The two fractions can be calculated from one another through the density of the materials.

The Volume fraction can be defined as V and the subscripts are dependent to the c, the composite material, $\mathrm{f}$, the fiber, and $\mathrm{m}$, the matrix or the epoxy. The volume fractions are a function of ratios of the volumes, $v$ of each constituent material. The volume fractions of the materials can be seen in Equation 1 and Equation 2.

Equation 1: Volume of Composite

$$
v_{c}=v_{f}+v_{m}
$$

Equation 2: Volume Fractions

$$
V_{f}=\frac{v_{f}}{v_{c}}, V_{m}=\frac{v_{m}}{v_{c}}
$$

The weight fraction can be defined as $\mathrm{W}$ and the subscripts are dependent to the c, the composite material, $\mathrm{f}$, the fiber, and $\mathrm{m}$, the matrix or the epoxy. The weight fractions are a function of ratio of the weights, $w$ of each constituent material. The weight fraction of the materials can be seen in Equation 3 and Equation 4. 
Equation 3: Weight of Composite

$$
w_{c}=w_{f}+w_{m}
$$

Equation 4: Weight Fractions

$$
W_{f}=\frac{w_{f}}{w_{c}}, W_{m}=\frac{w_{m}}{w_{c}}
$$

The relation of the volume fraction and the weight fraction was dependent of the density, $\rho$, of the composite, the matrix, and the fiber. The respective volume fraction can be converted through a ratio of densities; that can be seen in Equation 5. The equation was in its general form in terms of a place holder subscript, 1 , which can be replaced with either the fiber or matrix.

Equation 5: Generalized Equation of the Volume and Weight Fraction Relation

$$
V_{\iota}=\frac{\rho_{c}}{\rho_{\iota}} W_{\iota}
$$

The volume function was then used to calculate the Elastic Modulus, E, of the composite material in the longitudinal and lateral axes. That can be seen in Equation 6 and Equation 7. The subscripts 1 and 2 represent the axial and transverse axes of the material. The equations are in terms of the elastic modulus and the volume fractions of the constituent materials and also the Poisson's ratio, $v$, of the matrix.

Equation 6: Elastic Modulus of Composite in Longitudinal Direction

$$
E_{1}=V_{f} E_{f}+V_{m} E_{m}
$$

Equation 7: Elastic Modulus of Composite in Transverse Direction

$$
E_{2}=\frac{E_{f} E_{m}^{\prime}}{V_{f} E_{m}^{\prime}+V_{m} E_{f}\left(1-v_{m}^{2}\right)}, \text { where } E_{m}^{\prime}=\frac{E_{m}}{1-2 v_{m}^{2}}
$$

The ratio of the matrix and fiber can be checked to see if the volume fraction for the fiber was within limits to predict the composite strength. A critical fiber volume fraction, $\mathrm{V}_{\text {crit, }}$, that must not be exceeded to ensure strengthening of the fiber from the matrix, this can be seen Equation 8, Equation 9, and Equation 10. The critical fiber volume fraction has to be larger than the volume fiber that was calculated from the results of the volume fraction testing. This was calculated from the longitudinal strength of the composites, $\sigma_{\mathrm{cu}}$, the ultimate strength of the fibers, 
$\sigma_{\mathrm{fu}}$, the matrix stress at the fiber fracture strain, $\left(\sigma_{\mathrm{m}}\right)_{\varepsilon f^{*}}$, and the volume fraction of the fiber. The longitudinal strength of the composites can be calculated from experimental results of the LTM45 laminate only. The fiber and matrix stress can be calculated from the ratios of the elastic modulus of the constituent material and its stress; this can be seen in Equation 11. The ultimate composite failure stress can be used to calculate its constituent stress for the fiber and matrix.

Equation 8: Ultimate Stress of Composite Equation 1

$$
\sigma_{c u}=\sigma_{f u} V_{f}+\left(\sigma_{m}\right)_{\varepsilon f *}\left(1-V_{f}\right)
$$

Equation 9: Ultimate Stress of Composite Equation 2

$$
\sigma_{c u}=\sigma_{m}\left(1-V_{f}\right)
$$

Equation 10: Critical Fiber Volume Fraction Equation

$$
V_{c r i t}=\frac{\sigma_{m u}-\left(\sigma_{m}\right)_{\varepsilon f *}}{\sigma_{f u}-\left(\sigma_{m}\right)_{\varepsilon f *}}
$$

Equation 11: Stress/Elastic Modulus Ratio

$$
\frac{\sigma_{c}}{E_{c}}=\frac{\sigma_{f}}{E_{f}}=\frac{\sigma_{m}}{E_{m}}
$$

\subsection{Analysis of Elastic Modulus of Composite Sandwich Panel}

The Elastic Modulus of a composite sandwich panel can be calculated by combining the constituent Elastic Modulus of the face sheet and the core. It can be calculated by using the extensional matrix, A; this can be seen in its generalized form in Equation 12. The equation can be calculated from the reduced stiffness matrix, $\bar{Q}$ and the layer of the laminate from the center of the sandwich, $h_{k}$. The height from the center of the sandwich can be defined in Figure 62 . ${ }^{\text {vii }}$ The laminate can be defined as the center laminate as the foam core and two laminates on both sides of the core. The reduced stiffness matrix can be calculated from the stress tensor of each constituent material, which uses the elastic modulus of the material. The equation would have two reduced stiffness matrix, one for LTM45 and one for the foam. 
Equation 12: Generalized Form of the Extensional Stiffness Matrix

$$
A_{i j}=\sum_{k=1}^{n}\left(\overline{Q_{i j}}\right)_{k}\left(h_{k}-h_{k-1}\right)
$$

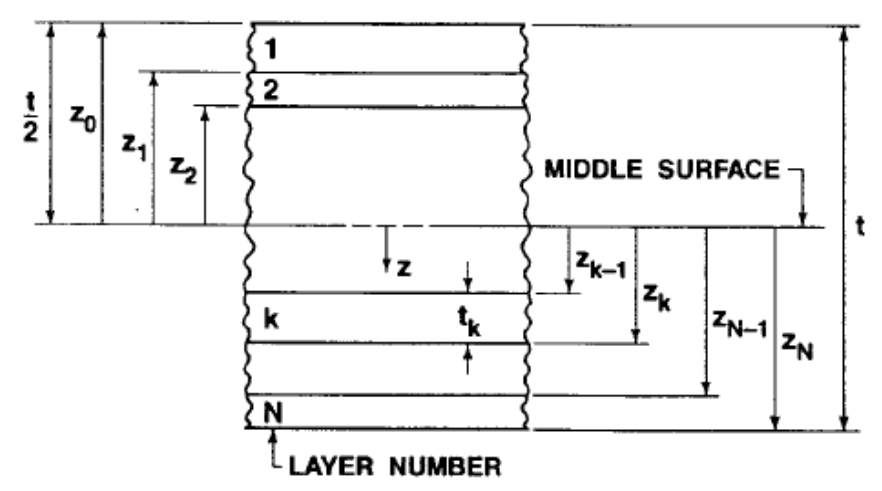

Figure 62: Example of laminate heights (Courtesy of Agarawal)

The Elastic Modulus of the overall sandwich composite panel can be obtained. The extensional stiffness matrix has another equation form in terms of just the overall Elastic Modulus; this can be seen in Equation 13. The equation was in terms of the overall Elastic Modulus, E, and the overall thickness of the sandwich panel, t, and the overall Poisson's Ratio, v. Since it was one equation and has two unknowns, another equation was needed. The general constitutive equation for force can be used as the second equation; this can be seen in Equation 14. The equation contains the forces, $\mathrm{N}$, and the strains, $\varepsilon$, see on the plate. Experimental data can be used for the forces and strains to calculate the Elastic Modulus and Poisson's Ratio of the composite sandwich panel

Equation 13: Alternate Extensional Stiffness Matrix

$$
[A]=\left[\begin{array}{ccc}
\frac{E t}{1-v^{2}} & \frac{v E t}{1-v^{2}} & 0 \\
\frac{v E t}{1-v^{2}} & \frac{E t}{1-v^{2}} & 0 \\
0 & 0 & \frac{E t}{2(1-v)}
\end{array}\right]
$$

Equation 14: General Constitutive Equation for forces with a symmetric plate

$$
\{N\}=[A]\left\{\varepsilon^{0}\right\}
$$




\section{Experimental Results and Discussion}

All experimental testing and results will discussed in this chapter in detail. The mechanical characteristics of the material were tested, such as the volume fraction of the face sheets and the material characteristics of the core, the face sheet, and the overall composite sandwich panels. The effects and trends of changing the DADs thickness on the failure characteristics of the composite sandwich panel would be discussed. Lastly, the fatigue characteristics and trends of the DADs on the composite sandwich panel will be discussed.

\subsection{Mechanical Characteristics of Material/Specimen}

\subsubsection{Volume Fraction of Face Sheets}

The volume fraction of the face sheets with and without a film adhesive was tested and analyzed. The specimen procedure and manufacturing were discussed in 3.6 Testing Procedure of Volume Fraction of Face Sheet. The results from the burn test for both test groups can be seen in Table 7. Six specimens were tested for each group and the results were averaged for further analysis. From the results, it can be seen that adding the film adhesive increases the resin content of the laminate by $6 \%$ from $54.8 \%$ to $61.1 \%$ in weight of the specimens.

Table 7: Results from Volume Fraction Testing

\begin{tabular}{|c|c|c|c|c|}
\hline & \multicolumn{2}{|c|}{ Without Film Adhesive } & \multicolumn{2}{c|}{ With Film Adhesive } \\
\hline Specimen & $\mathbf{W t}_{\mathbf{i}} \mathbf{( g )}$ & $\mathbf{W i}_{\mathbf{f}} \mathbf{( g )}$ & $\mathbf{W t}_{\mathbf{i}} \mathbf{( g )}$ & $\mathbf{W i}_{\mathbf{f}} \mathbf{( g )}$ \\
\hline 1 & 1.4 & 0.8 & 0.9 & 0.5 \\
\hline 2 & 1.4 & 0.8 & 0.9 & 0.5 \\
\hline 3 & 1.6 & 0.8 & 0.9 & 0.6 \\
\hline 4 & 1.4 & 0.8 & 0.9 & 0.5 \\
\hline 5 & 1.2 & 0.6 & 0.9 & 0.6 \\
\hline 6 & 1.4 & 0.8 & 0.9 & 0.6 \\
\hline Average & $\mathbf{1 . 4}$ & $\mathbf{0 . 7 6 7}$ & $\mathbf{0 . 9}$ & $\mathbf{0 . 5 5}$ \\
\hline
\end{tabular}


These values were not really that helpful until converted to volume fractions. The averaged weights of the fiber and the matrix, with the use of the average dimensions, the volume fractions were calculated. The densities of the materials were obtained by using the dimensions of the specimens and the weights of the materials for the densities. The densities for the case the film adhesive of the composites were $6.57 \mathrm{~g} / \mathrm{cm}^{3}$, for the LTM45 was $5.60 \mathrm{~g} / \mathrm{cm}^{3}$, and for the matrix were $1.24 \mathrm{~g} / \mathrm{cm}^{3}$. The volume fractions were than calculated; this can be seen in Table 8 . The volume fraction showed that the matrix content drops by $7.5 \%$, opposite from the $6 \%$ increase in weight. This could be due to the accuracy of the volume fraction because it was based on the densities and the volumes used for the calculations. A constant volume obtained from the test specimen was used for both the fiber and matrix volumes. The table also shows the critical volume fraction for the fiber of the LTM45. The obtained volume fraction for the fiber was found to be $68.5 \%$. The volume fraction of the composite without a film adhesive was within this value while the volume fraction of the composite with a film adhesive was 3.2\% above the value. This means that the composite had exceeded the strain hardening and plastic flow of the matrix. This made the composite more brittle than the optimum conditions that it was manufactured for. Since the research was to study the effects between the fastener and hole of the composite sandwich panel, the volume fraction of the fiber was suitable for testing.

Table 8: Volume Fractions with and without the film adhesive

\begin{tabular}{|c|c|}
\hline $\mathrm{V}_{\mathrm{f}}$ w/o Film Adhesive & $64.2 \%$ \\
\hline $\mathrm{V}_{\mathrm{m}} \mathrm{w} / \mathrm{o}$ Film Adhesive & $35.7 \%$ \\
\hline $\mathrm{V}_{\mathrm{f}} \mathrm{W} /$ Film Adhesive & $71.7 \%$ \\
\hline $\mathrm{V}_{\mathrm{m}} \mathrm{w} /$ Film Adhesive & $28.3 \%$ \\
\hline $\mathrm{V}_{\text {crit }}$ & $68.5 \%$ \\
\hline
\end{tabular}




\subsubsection{Material Properties}

The material properties of the composite sandwich panel were tested and analyzed. The first material property that was tested was the Elastic Modulus and the Poisson's Ratio of the core, FR-6710, and the face sheet, LTM45. The core was tested by cutting the specimens of the core into dimensions of 2" x 2” x 0.5 ”. The face sheet was tested by following ASTM standards on calculating the material properties of the composite, ASTM D3410.

The material properties of the core, FR-6710 were tested and analyzed. The test apparatus can be seen in Figure 64. The Elastic Modulus and the Poisson's Ratio of the material were calculated from the stress/strain curve of the test specimens; this can be seen from Figure 63. The figure shows that the five test specimens had similar slopes, showing that the Elastic Modulus for the foam was consistent with one another. The Poisson's Ratio was calculated by adding a strain gage on the horizontal direction to calculate the value. The results can be seen in Table 9.

Table 9: Material Characteristics of Last-A-Foam FR-6710

\begin{tabular}{|c|c|c|c|c|c|c|}
\hline & $\begin{array}{l}\text { Compressive } \\
\text { Strength (psi) }\end{array}$ & $\begin{array}{c}\text { Percent } \\
\text { Difference } \\
(-)\end{array}$ & $\begin{array}{c}\text { Elastic } \\
\text { Modulus } \\
\text { (psi) }\end{array}$ & $\begin{array}{c}\text { Percent } \\
\text { Difference } \\
(-)\end{array}$ & $\begin{array}{c}\text { Poisson's } \\
\text { Ratio (-) }\end{array}$ & $\begin{array}{c}\text { Percent } \\
\text { Difference } \\
(-)\end{array}$ \\
\hline Manufacturer & 340 & \multirow[b]{2}{*}{$-20.6 \%$} & 11,240 & \multirow[b]{2}{*}{$-34.8 \%$} & 0.30 & \multirow[b]{2}{*}{$-63.3 \%$} \\
\hline $\begin{array}{l}\text { Experimental } \\
\text { Testing }\end{array}$ & 270 & & 7,330 & & 0.11 & \\
\hline
\end{tabular}

The property of the foam was analyzed and it decreased by $20.6 \%$ for the compressive strength and about $34.8 \%$ for the elastic modulus for the material. The decrease in compressive strength and elastic modulus could be due to the way the specimens were tested. The testing procedure for the foam can be different from how the manufacturer tested the foam causing the significant difference in material characteristics. The decrease in material property can also be due to the degradation of the material from being stored too long after purchasing and receiving the material. Being stored in an uncontrolled environment can affect the material characteristics of the foam, for example, storing the foam in the sun would make it susceptible to UV 
degradation. For comparison with the FEM, the experimental results of the material characteristics were used for comparison between experimental and numerical solution.

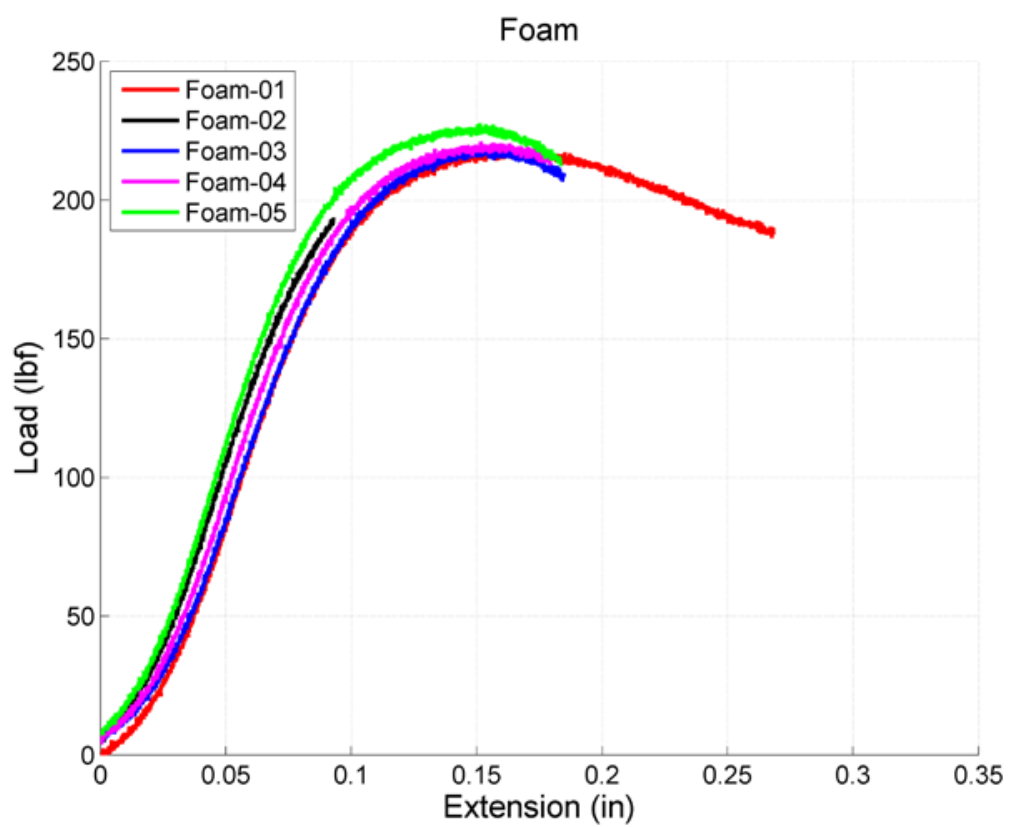

Figure 63: Load/Extension for Last-A-Foam FR-6710

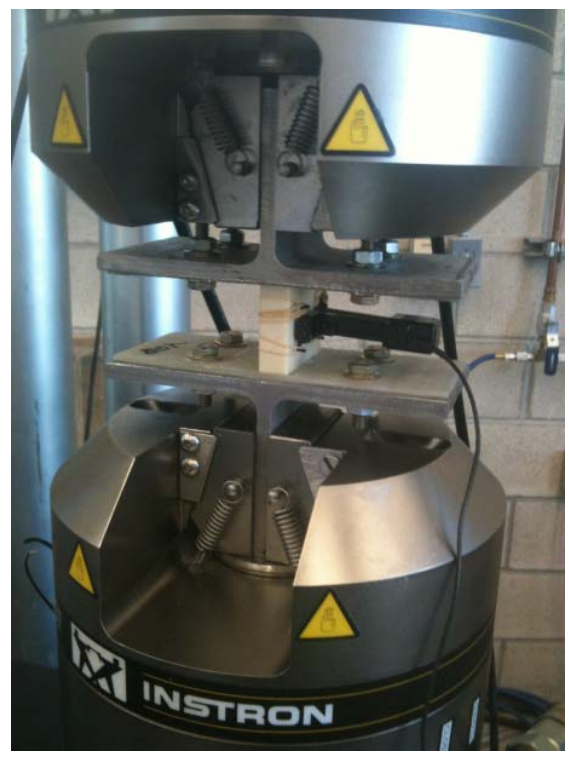

Figure 64: Foam Material Property Testing 
The material properties of the face sheet, LTM45 were tested and analyzed. The test apparatus can be seen in Figure 65. The Elastic Modulus and the Poisson’s Ratio of the material were calculated from the stress/strain curve of the test specimens. The Poisson's Ratio was calculated by adding a strain gage on the horizontal direction to calculate the value. The results can be seen in Table 10.

Table 10: Material Characteristics of LTM45

\begin{tabular}{|c|c|c|c|c|c|c|}
\hline & $\begin{array}{l}\text { Compressive } \\
\text { Strength (psi) }\end{array}$ & $\begin{array}{c}\text { Percent } \\
\text { Difference } \\
(-)\end{array}$ & $\begin{array}{l}\text { Elastic } \\
\text { Modulus } \\
\text { (psi) }\end{array}$ & $\begin{array}{c}\text { Percent } \\
\text { Difference } \\
(-)\end{array}$ & $\begin{array}{c}\text { Poisson's } \\
\text { Ratio (-) }\end{array}$ & $\begin{array}{c}\text { Percent } \\
\text { Difference } \\
(-)\end{array}$ \\
\hline Manufacturer & 214,000 & \multirow[b]{2}{*}{$-35.0 \%$} & $17,260,000$ & \multirow[b]{2}{*}{$-58.6 \%$} & (N/A) & \multirow[b]{2}{*}{ N/A } \\
\hline $\begin{array}{l}\text { Experimental } \\
\text { Testing }\end{array}$ & 130,000 & & $7,149,000$ & & 0.11 & \\
\hline
\end{tabular}

The material properties of LTM45 were obtained from the manufacturer and tested to validate the data. From the table, the material properties of the material decreased by $35.0 \%$ for the compressive strength and about $58.6 \%$ for the elastic modulus of the material. This decrease in material property can be due to the degradation of the material. The material was donated by Boeing over three years ago and was stored in a freezer that barely meets the cooling standards of the carbon. When the material was donated from Boeing, it was considered by the FAA to not meet its specifications, but it was still suitable for research usage. The values obtained from experimental testing show that the material properties of the LTM45 were still adequate for the research because compared to the foam the material was infinitely stiff and could handle the objectives of the thesis. Just like the foam, the experimental results of LTM45 were used for the material characteristics for the FEM. 


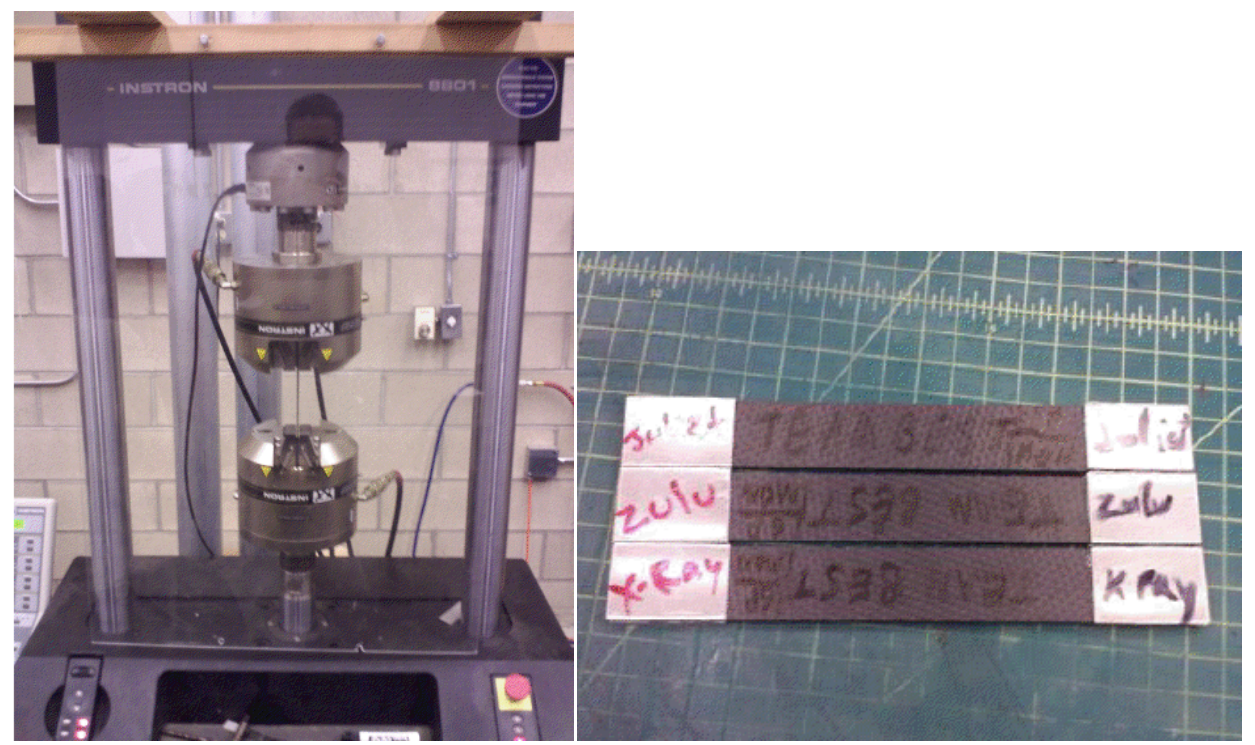

Figure 65: Test Specimens for LTM45

The next material property that was tested was the overall Elastic Modulus and the Poisson's Ratio of the whole composite sandwich panel; the results can be seen in Figure 66. The specimens plotted show the stress/strain curve of the specimens manufactured from 3.7 Specimen Preparation for Material Properties Testing. The results show that stress/strain curve of the specimens were only the linear part of the curve to be able to calculate the true Elastic Modulus of the overall composite sandwich panel without any notches (holes). The results can be seen in Table 11.

The theoretical results of the Elastic Modulus were calculated using the equations from 5.2 Analysis of Elastic Modulus of Composite Sandwich Panel. The typical failure of the test specimens, which can be seen in Figure 67, happens in the wood part of the core. The wood section of the core starts to fracture and it propagates into the foam section of the core which was then transferred to the face sheets. When the fracture reaches the face sheets, it starts to delaminate from the core. 


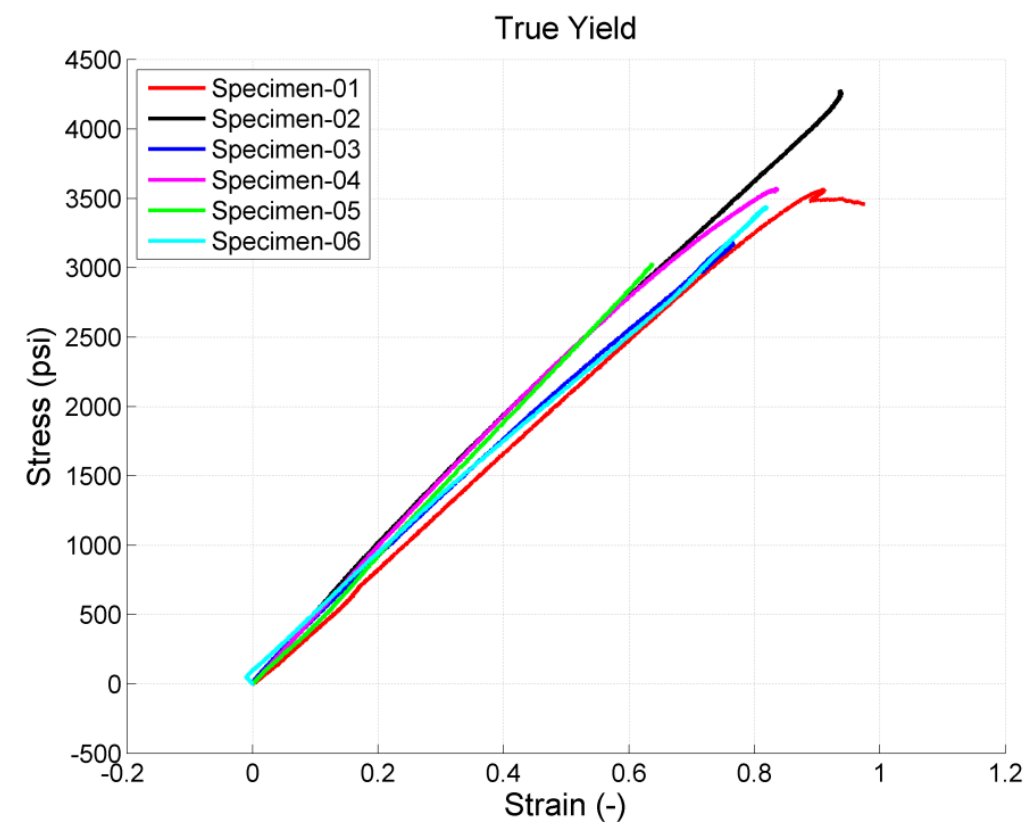

Figure 66: Stress/Strain of Test Specimens without Notches

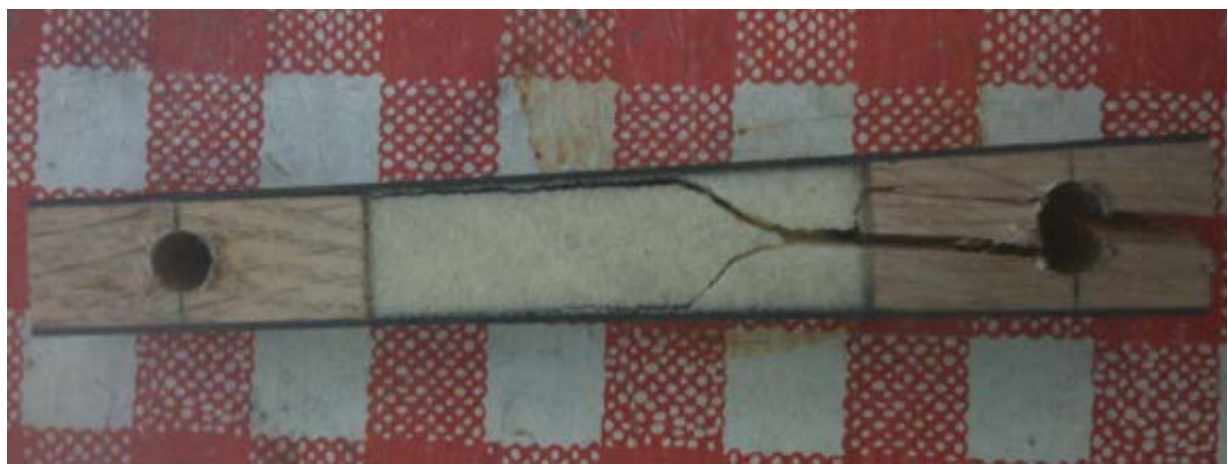

Figure 67: Failure of overall material property specimens

Table 11: Elastic Modulus of Composite Sandwich panel

\begin{tabular}{|c|c|c|c|c|}
\cline { 2 - 4 } \multicolumn{1}{c|}{} & $\begin{array}{c}\text { Elastic } \\
\text { Modulus } \\
(\mathrm{psi})\end{array}$ & $\begin{array}{c}\text { Percent } \\
\text { Difference } \\
(-)\end{array}$ & $\begin{array}{c}\text { Poisson's } \\
\text { Ratio (-) }\end{array}$ & $\begin{array}{c}\text { Percent } \\
\text { Difference } \\
(-)\end{array}$ \\
\hline Theoretical & 62,650 & \multirow{2}{*}{$33.6 \%$} & 0.30 & \multirow{2}{*}{$28.7 \%$} \\
\cline { 1 - 2 } $\begin{array}{c}\text { Experimental } \\
\text { Testing }\end{array}$ & 41,600 & & 0.214 & \\
\hline
\end{tabular}

The result shows that the theoretical Elastic Modulus of the composite sandwich panel was 62,650 psi. This was calculated by assuming a Poisson's Ratio of 0.3 in the equations 
because the calculations from the equations gave a singular matrix. The experimental testing gave an Elastic Modulus of 41,600 psi and a Poisson's Ratio of 0.214. The theoretical results over predicted the material properties of the composite sandwich panel. This over prediction was due to the calculations were for laminates and not composite sandwich panels, but it can be assumed that the core can act as part of the laminate.

\subsection{Strain Rate/Control Group Results}

The strain rate for the static testing needed to be investigated first before investigating the effects of the DADs on the composite sandwich panel. The strain rates that were investigated were between the rates of $0.5 \mathrm{~mm} / \mathrm{min}$ to $1.5 \mathrm{~mm} / \mathrm{min}$ with a spacing of $0.25 \mathrm{~mm} / \mathrm{min}$. The results can be seen in Table 12. Five control group plates were manufactured and cut down to specimens. A random sampling from the five plates was chosen for each strain rate to make sure that the results were not biased from testing the strain rate from the same plate. The results showed that all five strain rates had similar failure load varying from 926 lbs to 1,011 lbs and had similar elastic modulus varying from 37,530 psi to 48,940 psi. Variance in the failure load and the elastic modulus was due to the manufacturing defects in the specimens. The results show that the strain rate did not greatly affect the characteristics of the panel, but it did show a variance in standard deviation for each case.

Table 12: Strain Rate Selection Results

\begin{tabular}{|c|c|c|c|c|}
\hline Rate & $\mathbf{P}_{\text {cr }}$ & Std Dev & E & Std Dev \\
\hline$(\mathrm{mm} / \mathrm{min})$ & $(\mathrm{lb})$ & $(-)$ & $(\mathrm{psi})$ & $(-)$ \\
\hline 0.5 & 1,011 & $4.91 \%$ & 45,170 & $4.21 \%$ \\
\hline 0.75 & 983 & $9.54 \%$ & 48,940 & $7.53 \%$ \\
\hline 1.0 & 934 & 36.51 & 37,530 & $51.92 \%$ \\
\hline 1.25 & 926 & $12.71 \%$ & 45,010 & $9.51 \%$ \\
\hline 1.5 & 940 & $7.34 \%$ & 46,270 & $5.03 \%$ \\
\hline
\end{tabular}


The table shows that the slowest rate, $0.5 \mathrm{~mm} / \mathrm{min}$ had the lowest standard deviation for both failure load and elastic modulus. This rate was used for all static testing because it gave the best standard deviations compared to the other rates translating a better accuracy to future tests; this can be seen in Figure 68. The results show that five of the six test specimens were within one standard deviation from the mean, while the first test specimen was considered an outlier, this being represented with an $\mathrm{X}$ as a symbol.

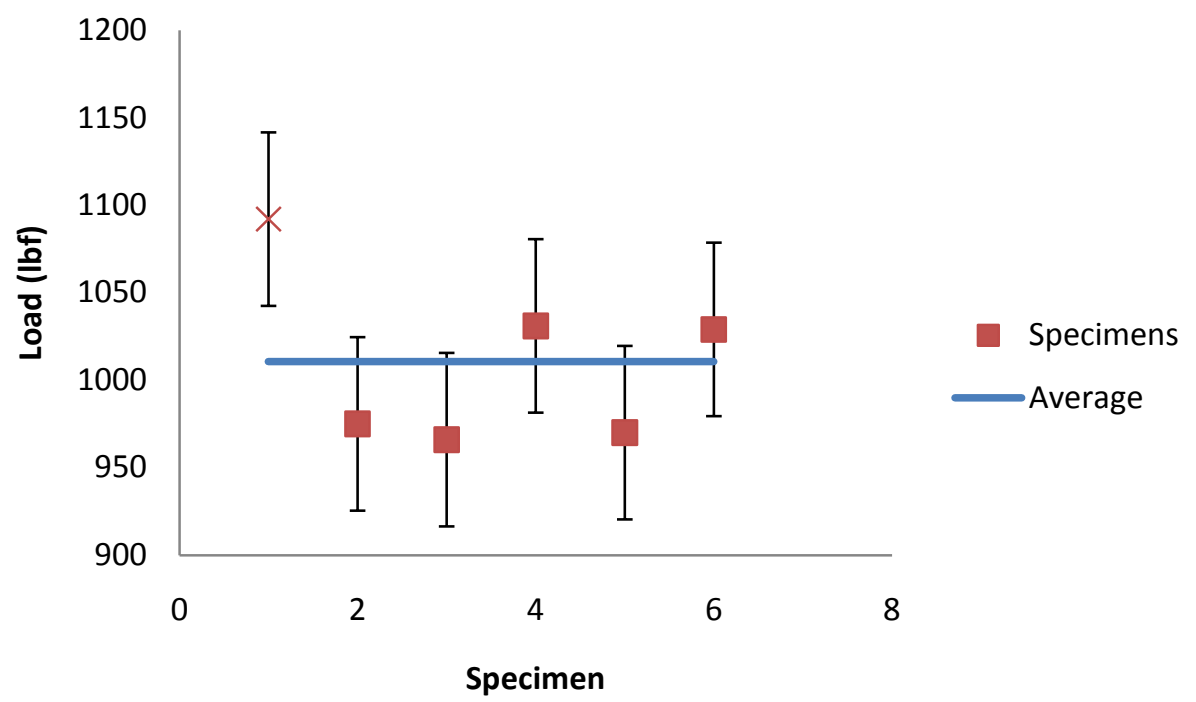

Figure 68: Standard Deviation for $0.5 \mathrm{~mm} / \mathrm{min}$

The results for the case, such as extension, stiffness, elastic modulus, and yield stress can be seen in Table 13. The outlier was highlighted in yellow for the table. The table shows that the load, the yield stress, and the extension were within $5 \%$ of one other in standard deviation meaning that the specimens are failing similarly to one another. This was very useful for fatigue testing because the yield stress or the first drop shows consistency to set the extension from experimental testing as the failure criterion for fatigue. 
Table 13: Results for 0.5 mm/min Strain Rate

\begin{tabular}{|c|c|c|c|c|c|c|}
\hline Test \# & $\begin{array}{c}\text { Test } \\
\text { Sample }\end{array}$ & Load & $\begin{array}{c}\text { Yield } \\
\text { Stress }\end{array}$ & Extension & $\mathrm{K}$ & $\mathrm{E}$ \\
\hline 1 & $0225-01$ & 1091.5 & 954.84 & 0.0538 & 24598 & 43789 \\
\hline 2 & $0225-06$ & 974.0 & 853.71 & 0.0540 & 22920 & 40864 \\
\hline 3 & $0226-01$ & 967.0 & 855.31 & 0.0507 & 27390 & 49375 \\
\hline 4 & $0226-06$ & 1030.7 & 908.31 & 0.0527 & 22551 & 40441 \\
\hline 5 & $0227-01$ & 969.4 & 850.38 & 0.0585 & 30342 & 54194 \\
\hline 6 & $0227-06$ & 1027.6 & 893.31 & 0.0569 & 23909 & 42382 \\
\hline \multicolumn{2}{|c|}{ Average Load } & 1010.014 & 885.98 & 0.05443 & 25285.1 & 45174.2 \\
\hline \multicolumn{2}{|c|}{ Std Dev } & $4.89 \%$ & $4.67 \%$ & $5.21 \%$ & $11.92 \%$ & $12.11 \%$ \\
\hline
\end{tabular}

For the rest of the strain rates that were tested, the standard deviations for the remaining strain rates can be seen in Figure 69, Figure 70, Figure 71, and Figure 72. The experimental results such as extension, stiffness, elastic modulus, and yield stress can be seen in Table 14, Table 15, Table 16, and Table 17. As before, the figures show the outlier with an X marker and the specimens within one standard deviation have a square marker. The other strain rates have more outliers than the $0.5 \mathrm{~mm} / \mathrm{min}$ by having as much as eight outliers and at least four outliers.

The worst of the strain rates was the $1.0 \mathrm{~mm} / \mathrm{min}$ where eight of the nine test specimens were one standard deviation from the median. It also had the highest standard deviation for the ultimate failure load and the Elastic Modulus had the lowest of the five strain rates. 


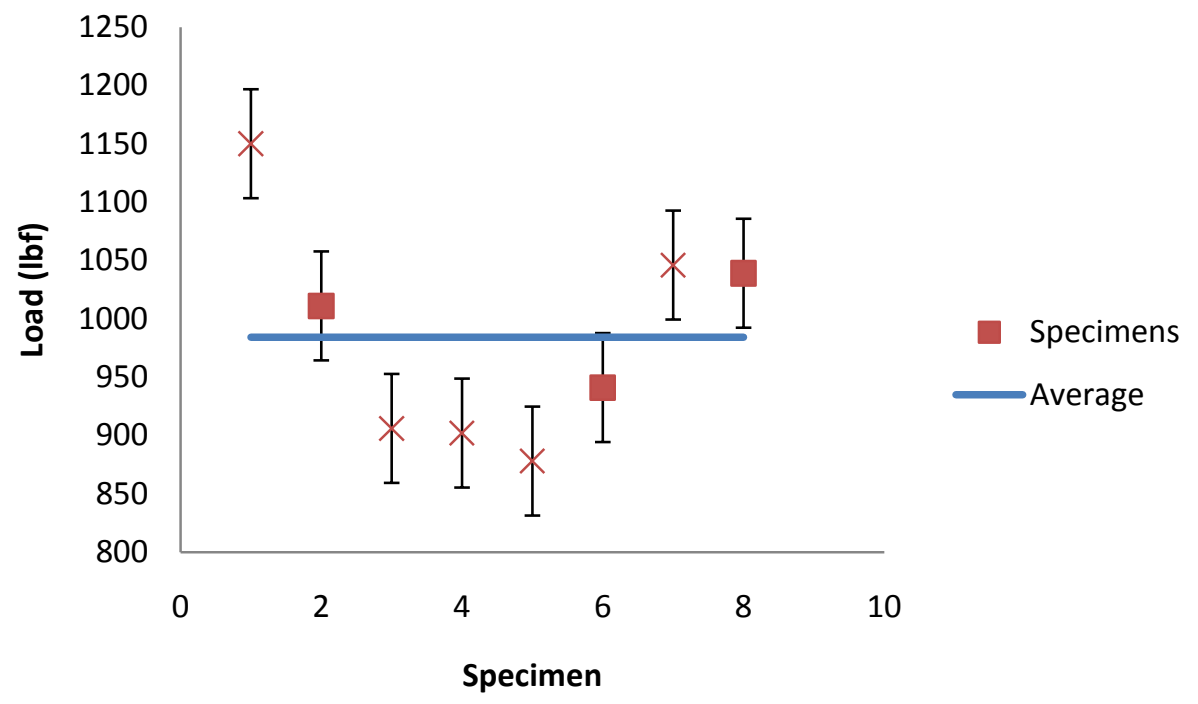

Figure 69: Standard Deviation for $0.75 \mathrm{~mm} / \mathrm{min}$

Table 14: Results for 0.75 mm/min Strain Rate

\begin{tabular}{|c|c|c|c|c|c|}
\hline Test \# & Test Sample & Load & Yield Stress & $\mathrm{K}$ & E \\
\hline 1 & 0225-02 & 1149 & 1001 & 30300 & 53965 \\
\hline 2 & $0225-07$ & 1010 & 891 & 28829 & 52063 \\
\hline 3 & 0226-02 & 905 & 801 & 28060 & 50670 \\
\hline 4 & 0226-07 & 901 & 784 & 25921 & 45082 \\
\hline 5 & 0227-02 & 877 & 765 & 24145 & 42984 \\
\hline 6 & 0227-07 & 940 & 821 & 26416 & 47155 \\
\hline 7 & 0305-01 & 1044 & 922 & 27133 & 48779 \\
\hline 8 & 0305-02 & 1038 & 915 & 28800 & 50824 \\
\hline \multicolumn{2}{|c|}{ Average Load } & 983 & 862 & 27450 & 48940 \\
\hline \multicolumn{2}{|c|}{ Std Dev } & $9.49 \%$ & $9.54 \%$ & $7.11 \%$ & $7.53 \%$ \\
\hline
\end{tabular}




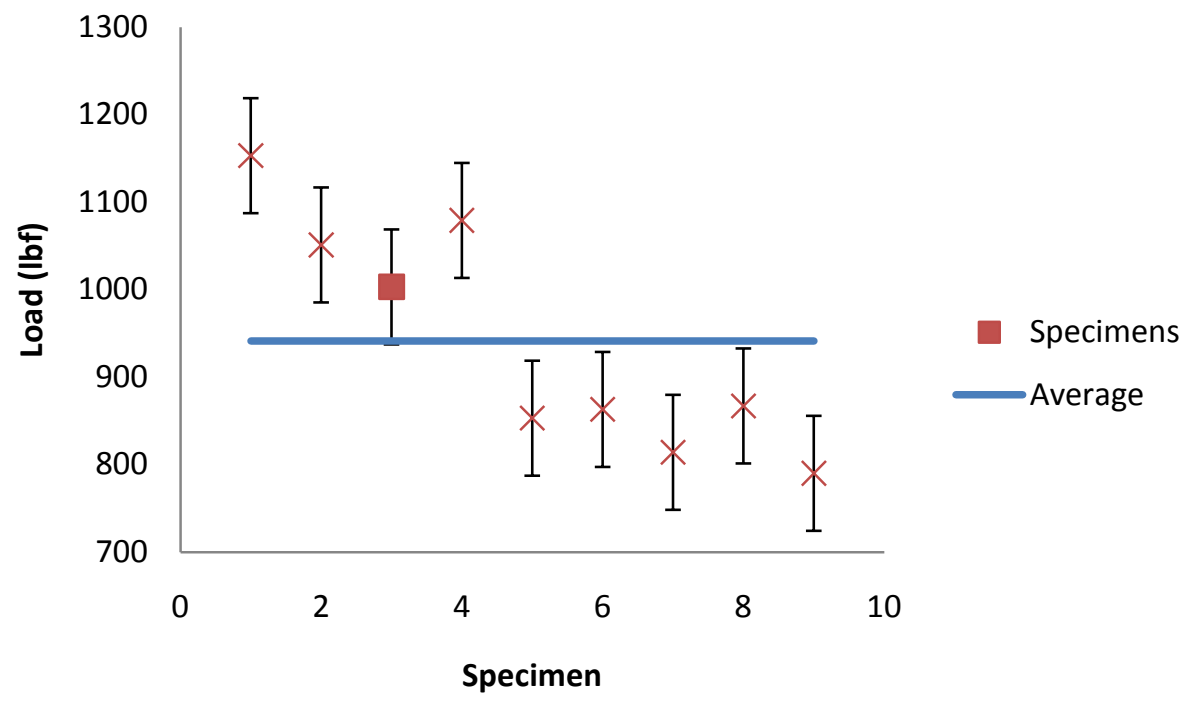

Figure 70: Standard Deviation for $1.0 \mathrm{~mm} / \mathrm{min}$

Table 15: Results for 1.0 mm/min Strain Rate

\begin{tabular}{|c|c|c|c|c|c|}
\hline Test \# & $\begin{array}{c}\text { Test } \\
\text { Sample }\end{array}$ & Load & $\begin{array}{c}\text { Yield } \\
\text { Stress }\end{array}$ & K & E \\
\hline 1 & $0225-03$ & 1153 & 1004 & 30549 & 54272 \\
\hline 2 & $0225-08$ & 1052 & 919 & 27990 & 49681 \\
\hline 3 & $0226-03$ & 1002 & 883 & 26295 & 47176 \\
\hline 4 & $0226-08$ & 1076 & 94 & 27533 & 4875 \\
\hline 5 & $0227-03$ & 853 & 748 & 28185 & 50366 \\
\hline 6 & $0227-08$ & 861 & 752 & 19646 & 34941 \\
\hline 7 & $0305-03$ & 796 & 692 & 24581 & 43640 \\
\hline 8 & $0305-04$ & 865 & 749 & 28030 & 48665 \\
\hline 9 & $0305-07$ & 751 & 670 & 22741 & 4113 \\
\hline \multicolumn{2}{|c|}{ Average Load } & 934 & 657 & 26172 & 37525 \\
\hline \multicolumn{2}{|c|}{ Std Dev } & $14.93 \%$ & $36.15 \%$ & $12.72 \%$ & $51.92 \%$ \\
\hline
\end{tabular}




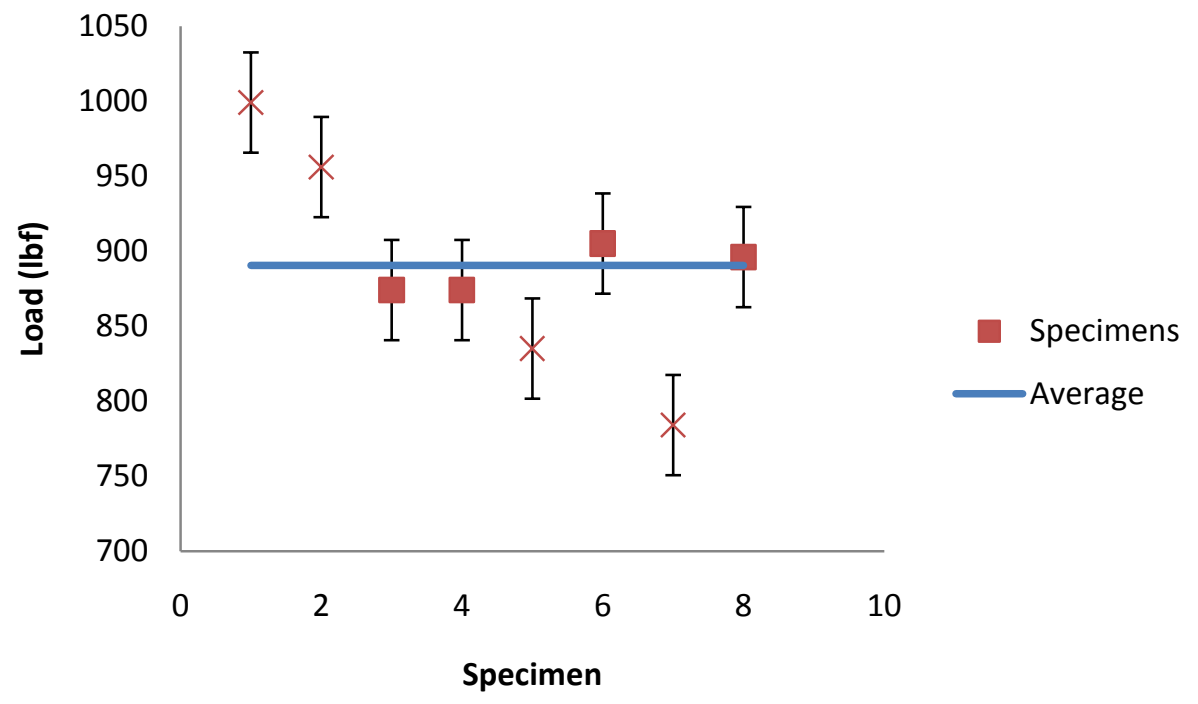

Figure 71: Standard Deviation for $1.25 \mathrm{~mm} / \mathrm{min}$

Table 16: Results for 1.25 mm/min Strain Rate

\begin{tabular}{|c|c|c|c|c|c|}
\hline Test \# & Test Sample & Load & Yield Stress & K & E \\
\hline 1 & $0225-04$ & 997 & 866 & 28798 & 51134 \\
\hline 2 & $0225-09$ & 954 & 832 & 22460 & 40020 \\
\hline 3 & $0226-04$ & 874 & 768 & 22835 & 40915 \\
\hline 4 & $0226-09$ & 1167 & 1017 & 28453 & 50613 \\
\hline 5 & $0227-04$ & 833 & 731 & 25978 & 46149 \\
\hline 6 & $0227-09$ & 904 & 794 & 26069 & 46526 \\
\hline 7 & $0305-05$ & 784 & 679 & 24287 & 42402 \\
\hline 8 & $0305-06$ & 896 & 796 & 23737 & 42331 \\
\hline \multicolumn{2}{|c|}{ Average Load } & 926 & 810 & 25327 & 45011 \\
\hline \multicolumn{2}{|c|}{ Std Dev } & $12.71 \%$ & $12.51 \%$ & $9.54 \%$ & $9.51 \%$ \\
\hline
\end{tabular}




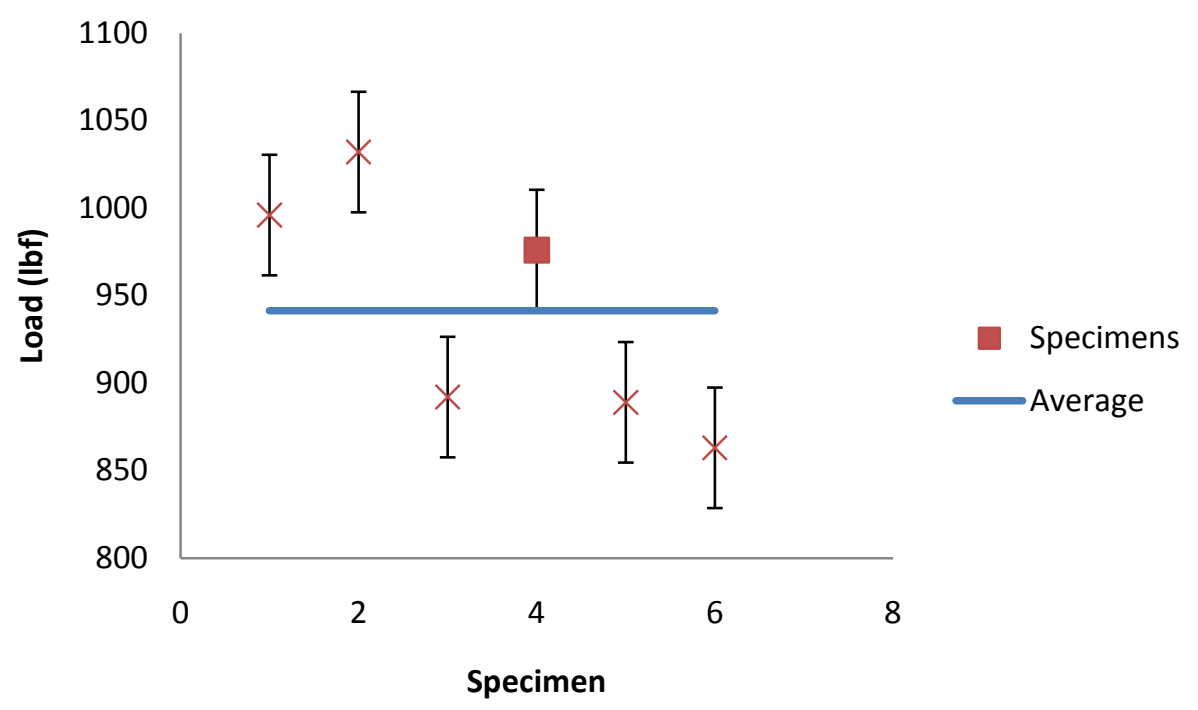

Figure 72: Standard Deviation for $1.5 \mathrm{~mm} / \mathrm{min}$

Table 17: Results for $1.5 \mathrm{~mm} / \mathrm{min}$ Strain Rate

\begin{tabular}{|c|c|c|c|c|c|}
\hline Test \# & Test Sample & Load & Yield Stress & K & E \\
\hline 1 & $0225-05$ & 995 & 865 & 26856 & 47394 \\
\hline 2 & $0225-10$ & 1031 & 904 & 26181 & 46929 \\
\hline 3 & $0226-05$ & 892 & 786 & 23578 & 42446 \\
\hline 4 & $0226-10$ & 974 & 850 & 25942 & 46118 \\
\hline 5 & $0227-05$ & 887 & 782 & 27550 & 49404 \\
\hline 6 & $0227-10$ & 862 & 754 & 25291 & 45292 \\
\hline \multicolumn{2}{|c|}{ Average Load } & 940 & 823 & 25900 & 46264 \\
\hline \multicolumn{2}{|c|}{ Std Dev } & $7.34 \%$ & $7.03 \%$ & $5.32 \%$ & $5.03 \%$ \\
\hline
\end{tabular}

The load/extension curves for the $0.5 \mathrm{~mm} / \mathrm{min}$ case had the best testing results because it had the least amount of test specimens that had a drop in the load/extension curve and continued after; this can be seen in Figure 73. The trend for the control group was that the specimen rose and then dropped and rose again, but dropped at a lower load than before. This was due to the fastener slowly moving along the composite sandwich panel. The second rise in the curve was caused by the face sheet re-fracturing as the fastener moves along it. This was also known as micro buckling where the fibers break one at a time instead of breaking at once. The leveling force seen at the end of the curve was when the fastener was "sawing" through the composite 
material with ease. This was when the panel already failed and was further discussed in 7. Types of Failure of Composite Sandwich Panels

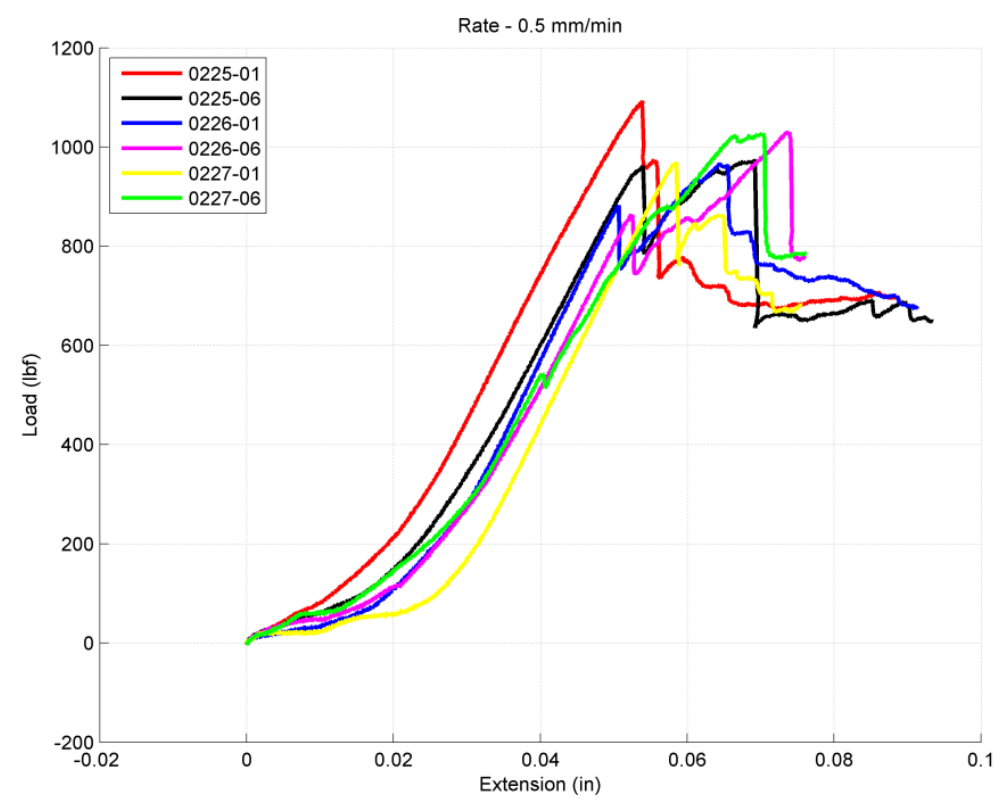

Figure 73: Load/Extension for $0.5 \mathrm{~mm} / \mathrm{min}$ Rate

The load versus extension curves for the remaining strain rates can be seen in Figure 74, Figure 75, Figure 76, and Figure 77. The four figures show that the remaining four strain rates had consistent test results by having similar slopes to one another. Just like the $0.5 \mathrm{~mm} / \mathrm{min}$ strain rate, the test specimens for each strain rate group rose and then dropped and rose again but dropped at a lower load than before. This was due to the fastener slowly moving along the composite sandwich panel. The second rise in the curve was caused by the face sheet refracturing as the fastener moved along it. This was also known as micro buckling where the fibers break one at a time instead of it breaking at once. The leveling force seen at the end of the curve was when the fastener was "sawing" through the composite material with ease. 


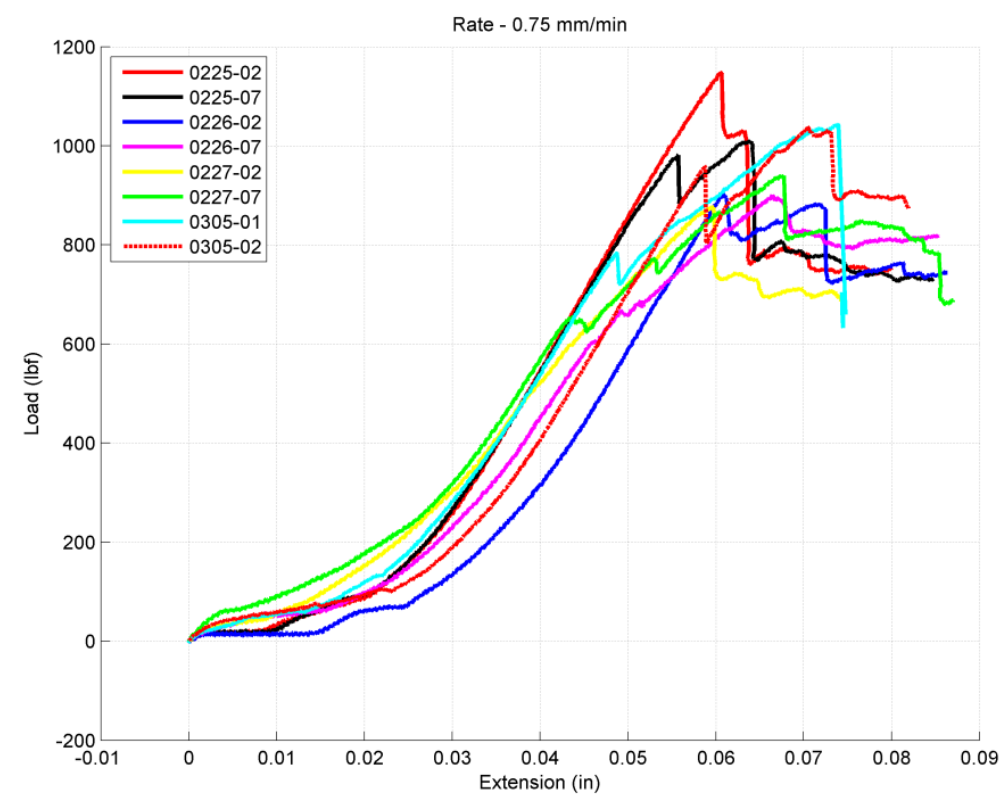

Figure 74: Load/Extension for 0.75 mm/min Rate

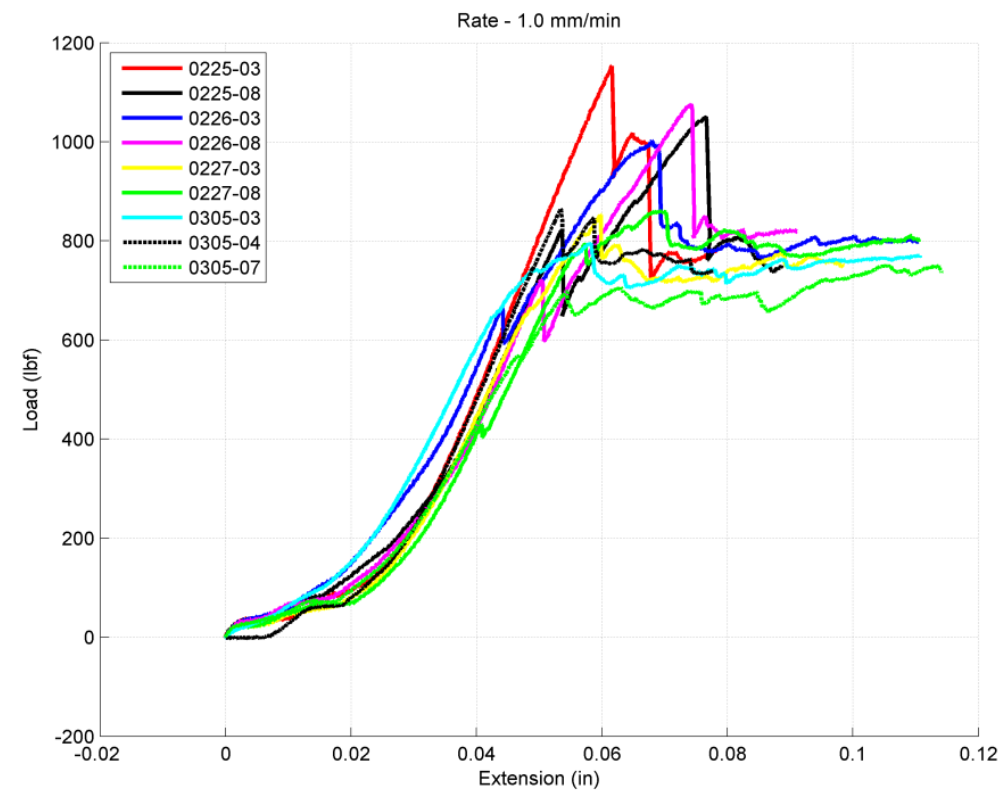

Figure 75: Load/Extension for $1.0 \mathrm{~mm} / \mathrm{min}$ Rate 


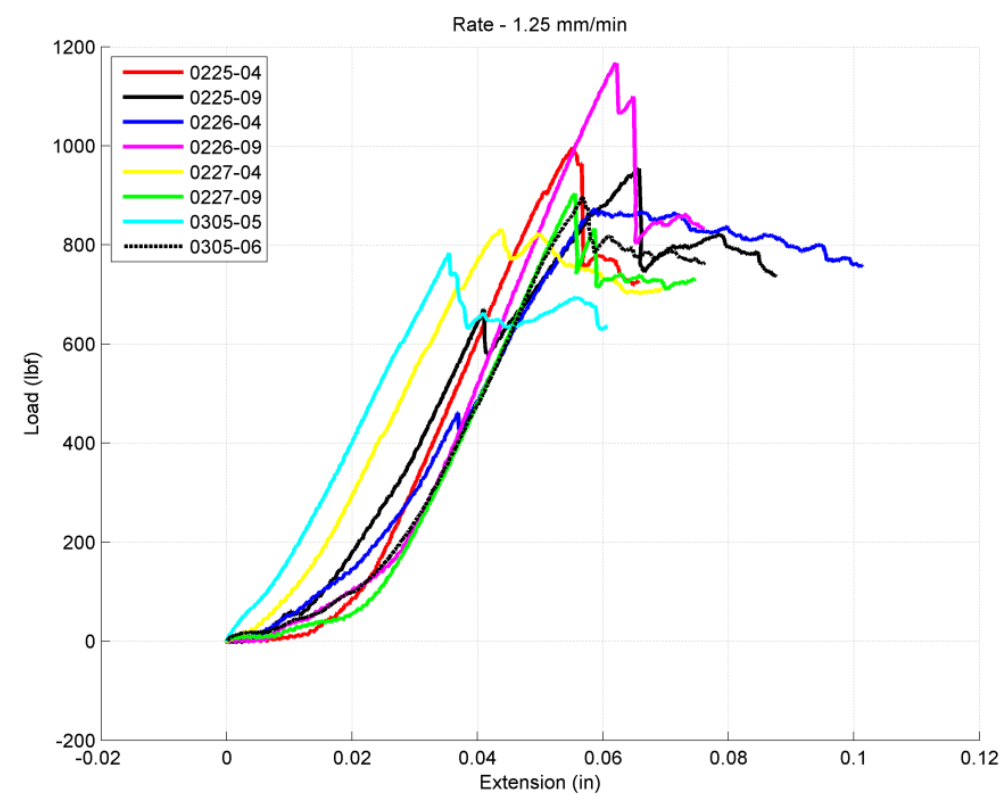

Figure 76: Load/Extension for 1.25 mm/min Rate

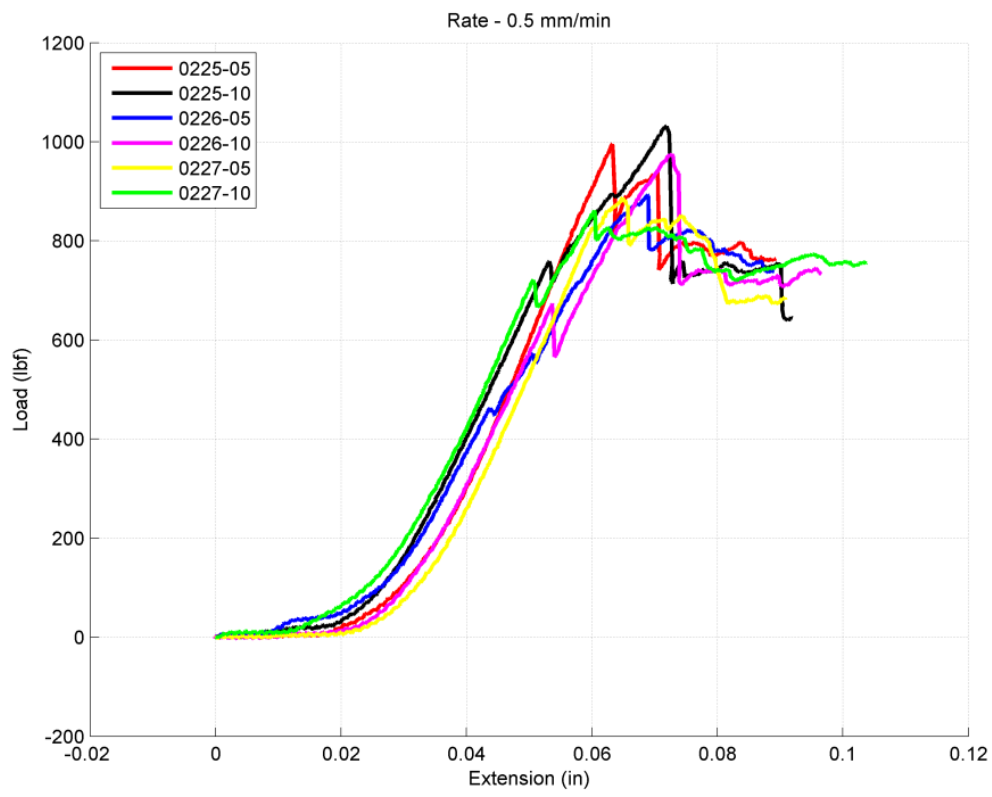

Figure 77: Load/Extension for 1.5 mm/min Rate 


\subsection{DAD Manufacturing Test Results}

Two types of DAD manufacturing techniques were tested. The first method was when the DADs were cured with the face sheet and the core at the same time. The second method was when the DAD was cured separately as a laminate and then cut and then inserted with the core and face sheet to cure for a second time. The two techniques were manufactured and tested to see which method was used for the DAD thickness testing.

The first method results can be seen in Table 18 and the standard deviation can be seen in Figure 78. The results show that the average ultimate load for the test group was 1,628 lbs and had a standard deviation of $2.49 \%$. The method shows that there were two outliers in the test group, the ninth and tenth test specimens. The rest of the test specimens were within one standard deviation of the mean and they were all close to the mean of the test group.

Table 18: Test Results for DAD Manufacturing Method 1

\begin{tabular}{|c|c|c|}
\hline Test \# & Test Sample & Load \\
\hline 1 & $0401-01$ & 1660.9 \\
\hline 2 & $0401-02$ & 1726.5 \\
\hline 3 & $0401-03$ & 1689.4 \\
\hline 4 & $0401-04$ & 1707.2 \\
\hline 5 & $0401-05$ & 1654.2 \\
\hline 6 & $0401-06$ & 1630.6 \\
\hline 7 & $0401-07$ & 1666.0 \\
\hline 8 & $0401-08$ & 1601.1 \\
\hline 9 & $0401-09$ & 1025.3 \\
\hline 10 & $0401-10$ & 1925.2 \\
\hline \multicolumn{2}{|c|}{ Average Load } & 1628.65 \\
\hline \multicolumn{2}{|c|}{ Std Dev W/ Outliers } & $2.49 \%$ \\
\hline
\end{tabular}




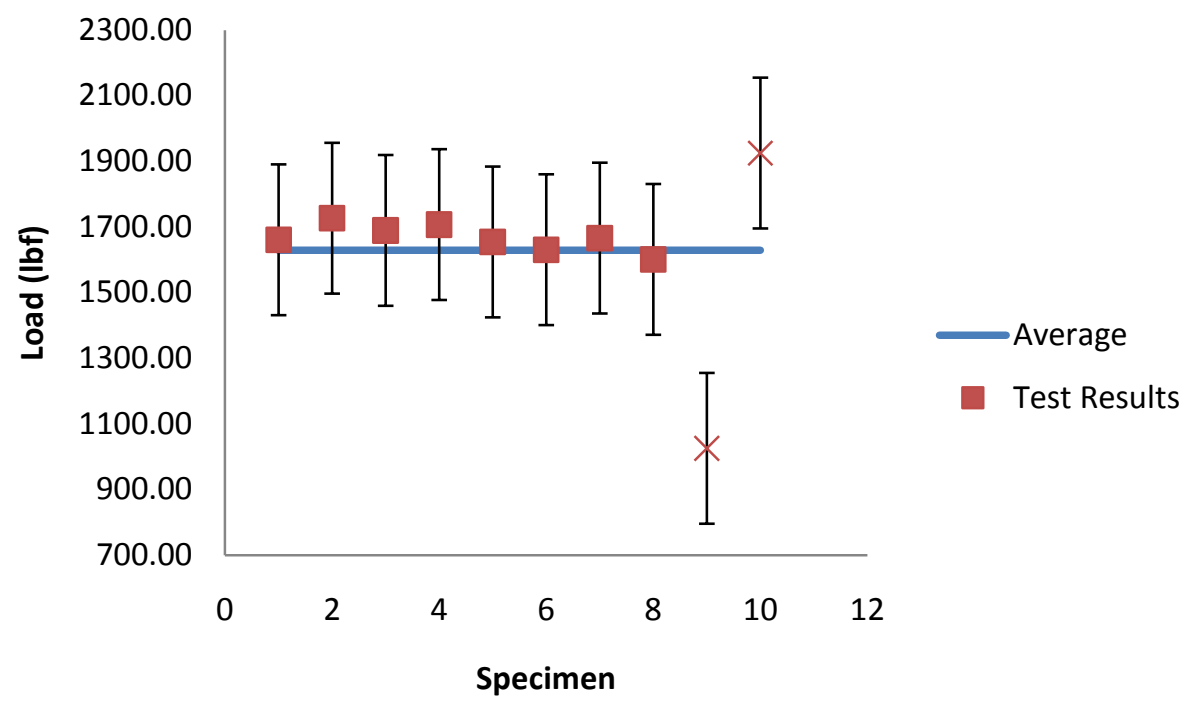

Figure 78: Standard Deviation for DAD Manufacturing Method 1

The first method results can be seen in Table 19 and the standard deviation can be seen in Figure 79.The results show that the average ultimate load for the test group was 1,942 lbs and had a standard deviation of $9.80 \%$. The method shows that there were three outliers in the test group, the fourth, fifth, and eighth test specimens. The rest of the test specimens were within one standard deviation of the mean and they were all close to the mean of the test group.

Table 19: Test Results for DAD Manufacturing Method 2

\begin{tabular}{|c|c|c|}
\hline Test \# & Test Sample & Load \\
\hline 1 & $0406-04$ & 2007 \\
\hline 2 & $0406-02$ & 1903 \\
\hline 3 & $0406-03$ & 2022 \\
\hline 4 & $0406-04$ & 1693 \\
\hline 5 & $0406-05$ & 1506 \\
\hline 6 & $0406-06$ & 1949 \\
\hline 7 & $0406-07$ & 1957 \\
\hline 8 & $0406-08$ & 1598 \\
\hline 9 & $0406-09$ & 1927 \\
\hline 10 & $0406-10$ & 1835 \\
\hline \multicolumn{2}{|c|}{ Average Load } & 1942.857 \\
\hline \multicolumn{2}{|c|}{ Std Dev W/ Outliers } & $9.80 \%$ \\
\hline
\end{tabular}




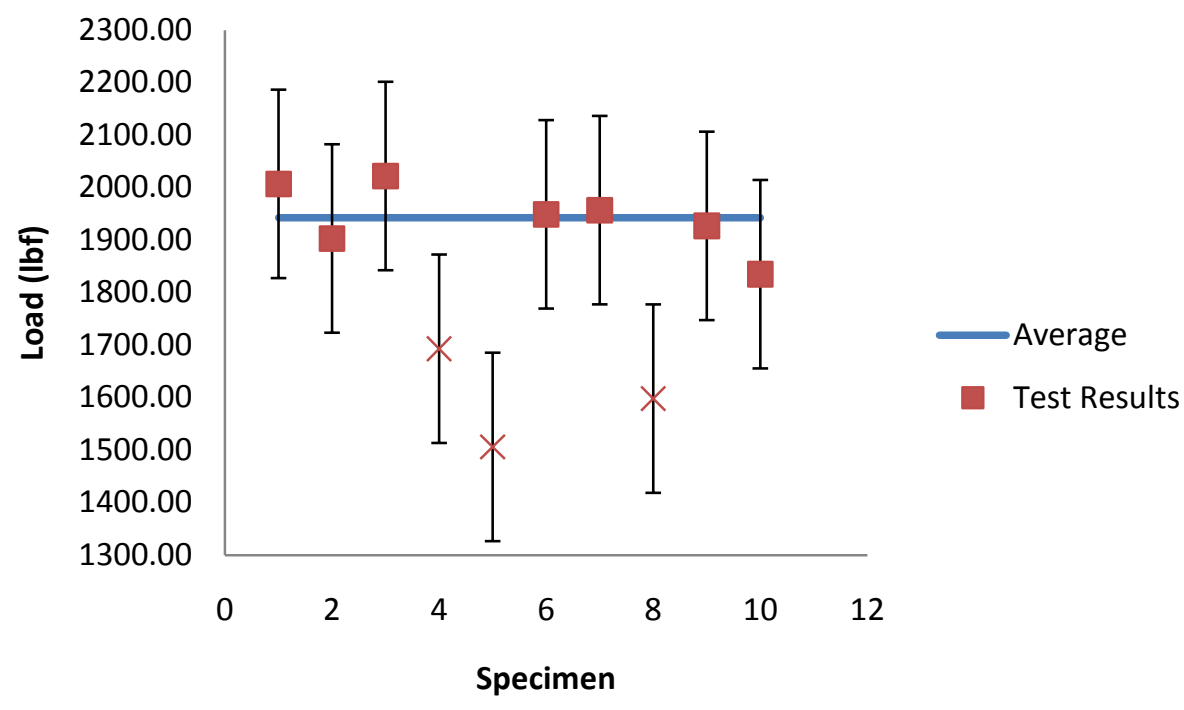

Figure 79: Standard Deviation for DAD Manufacturing Method 2

The test results show that excluding the outlier, the test specimens were close to or on the mean of the ultimate loads for the test groups. The results show that the second manufacturing method had a higher failure load, but had a higher standard deviation than the first manufacturing method. The second manufacturing method though shows better strength than the first method, the failure of the specimen was different from the ASTM standards; this can be seen in Figure 80 . The method had a delamination failure of the face sheet from the core between the two DADs and also buckling was seen on the test specimen. The failure was due to the lack of bonding between the face sheets to the DAD because it was cured before manufacturing. It seems that the cured DADs absorbed too much epoxy from the face sheet, showing the delamination between the two DADs. This was undesirable because it was more difficult to analyze the data with buckling being added to the analysis. 


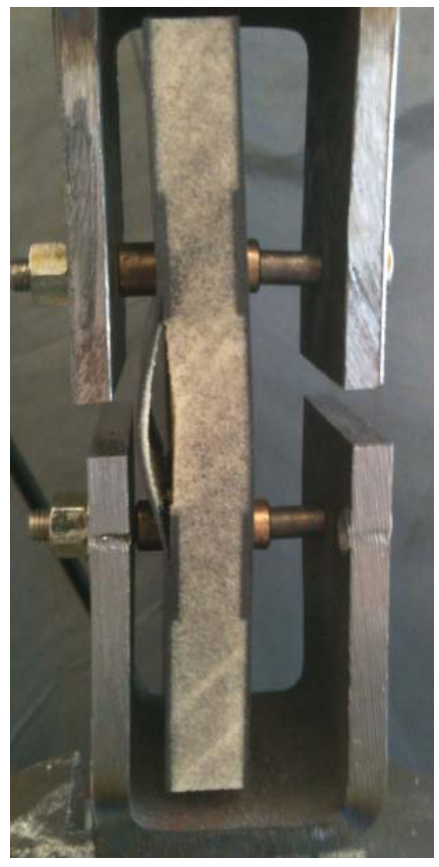

Figure 80: Failure of DAD Manufacturing Method 2

The first manufacturing method shows more of a bearing failure during the testing; this can be seen in Figure 81. It shows that the fastener was moving along the specimen and was creating a bearing failure around the fastener. The fastener pushed the DAD up the panel, causing the DAD to "pop” out of the face sheet and delaminate from the core. This created a fracture along the DAD on the face sheet. The failure will be talked about more in 7.1 Static Loading Failures. For the research, this manufacturing method for the DAD was used because it gave a better standard deviation, consistency and accuracy for the results. The specimen also gave a better failure that was defined by ASTM for this type of testing. 


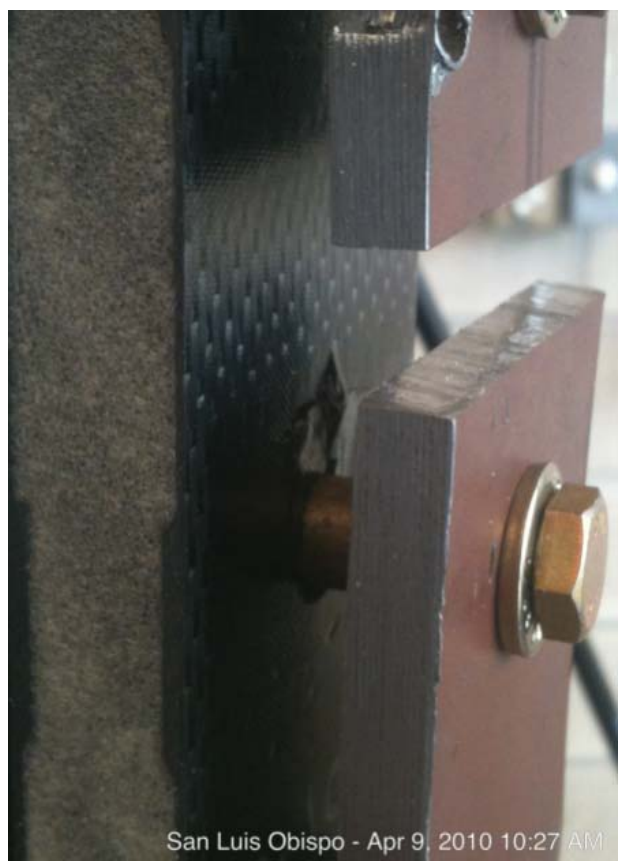

Figure 81: Failure of DAD Manufacturing Method 1

\subsection{DAD 1 Layer Results}

The first DAD static testing had a one layer thickness for the DAD; this can be seen in Figure 82. The test specimens had an increase in load of 36.8\% from the control group and an increase in Elastic Modulus by 55.9\%. The specimen results can be seen in Table 20.The table shows that the average ultimate load for the case was 1,381 lbs and an average Elastic Modulus of 66,736 psi. The increase in both failure load and elastic modulus was due to the addition of one extra layer of LTM45 to the area where the fastener interacted with the composite sandwich panel. The 55.9\% increase in the Elastic Modulus showed an interesting observation; an addition of one layer showed that the Elastic Modulus of the LTM45 was compounded around the hole. It meant that each layer of LTM45 evenly accounts for a third of the Elastic Modulus of the material because the face sheet will always carry a majority of the load than the core. 


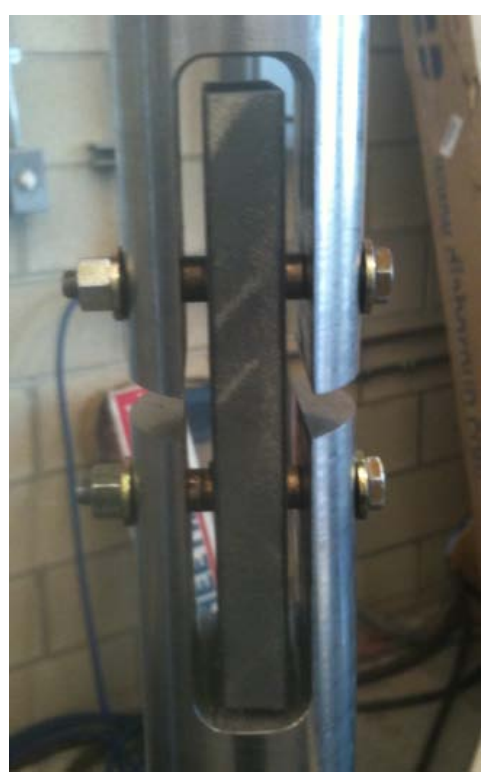

Figure 82: Test Specimen for DAD 1 Layer

The results also show that the standard deviation of the ultimate load was $9.37 \%$ and $10.62 \%$ for the Elastic Modulus. There were three outliers in the testing from the standard deviation which were test specimens two, nine and ten; seen in Figure 83. The figure shows the outlier with an $\mathrm{X}$ marker and the specimens within one standard deviation had a square marker.

Table 20: Test Results for DAD 1 Layer

\begin{tabular}{|c|c|c|c|c|c|c|}
\hline Test \# & $\begin{array}{c}\text { Test } \\
\text { Sample }\end{array}$ & $\begin{array}{c}\text { Ult } \\
\text { Load }\end{array}$ & Y Load & Extension & K & E \\
\hline 1 & $0509-01$ & 1319 & 1318 & 0.0435 & 43235 & 74639 \\
\hline 2 & $0509-02$ & 1801 & 1801 & 0.0569 & 45104 & 76548 \\
\hline 3 & $0509-03$ & 1640 & 1640 & 0.0630 & 45837 & 78258 \\
\hline 4 & $0509-04$ & 1415 & 1415 & 0.0552 & 36066 & 61829 \\
\hline 5 & $0509-05$ & 1287 & 1040 & 0.0534 & 33472 & 57682 \\
\hline 6 & $0509-06$ & 1438 & 1438 & 0.0590 & 45959 & 79753 \\
\hline 7 & $0509-07$ & 1177 & 1012 & 0.0539 & 38308 & 65813 \\
\hline 8 & $0509-08$ & 1426 & 1426 & 0.0624 & 42348 & 75285 \\
\hline 9 & $0509-09$ & 1166 & 1166 & 0.0510 & 39482 & 68134 \\
\hline 10 & $0509-10$ & 1160 & 1151 & 0.0575 & 39637 & 66736 \\
\hline \multicolumn{2}{|c|}{ Average } & 1383 & 1341 & 0.0556 & 40945 & 70468 \\
\hline $\begin{array}{r}\text { Std Dev W/ } \\
\text { Outliers }\end{array}$ & $9.37 \%$ & $19.09 \%$ & $10.27 \%$ & $10.43 \%$ & $10.62 \%$ \\
\hline \multicolumn{2}{|c|}{$\begin{array}{c}\text { Std Dev W/O } \\
\text { Outliers }\end{array}$} & $10.52 \%$ & $17.12 \%$ & $11.94 \%$ & $11.97 \%$ & $12.25 \%$ \\
\hline
\end{tabular}




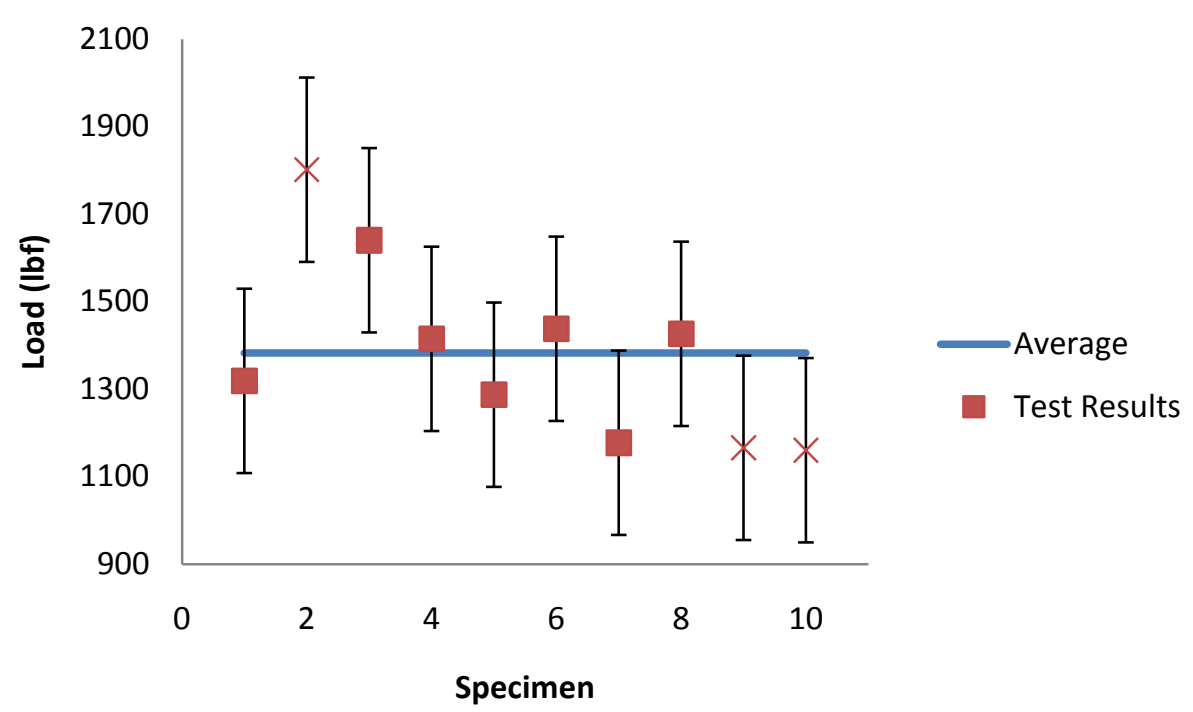

Figure 83: Standard Deviation for DAD 1 Layer

The load/extension curves for the case had consistent results; this can be seen in Figure 84. The trend for the group was that the specimen rises and then drops and continues dropping. This was due to the fastener slowly moving along the composite sandwich panel. This was due tothe insert delaminating from the core, taking the face sheet with it. The DAD "popped out” of the panel due to the force being exerted on it; this was talked about more in detail in 7.1 Static Loading Failures. The figure shows that the curves had similar slopes, showing that the Elastic Modulus and stiffness for the test group were similar to one another. All but one of the test specimens had their yield load, or the failure criterion having an extension of 0.05 in to 0.06 in. The first test specimen failed earlier, about ten thousandths of an inch, than the rest of the specimen. 


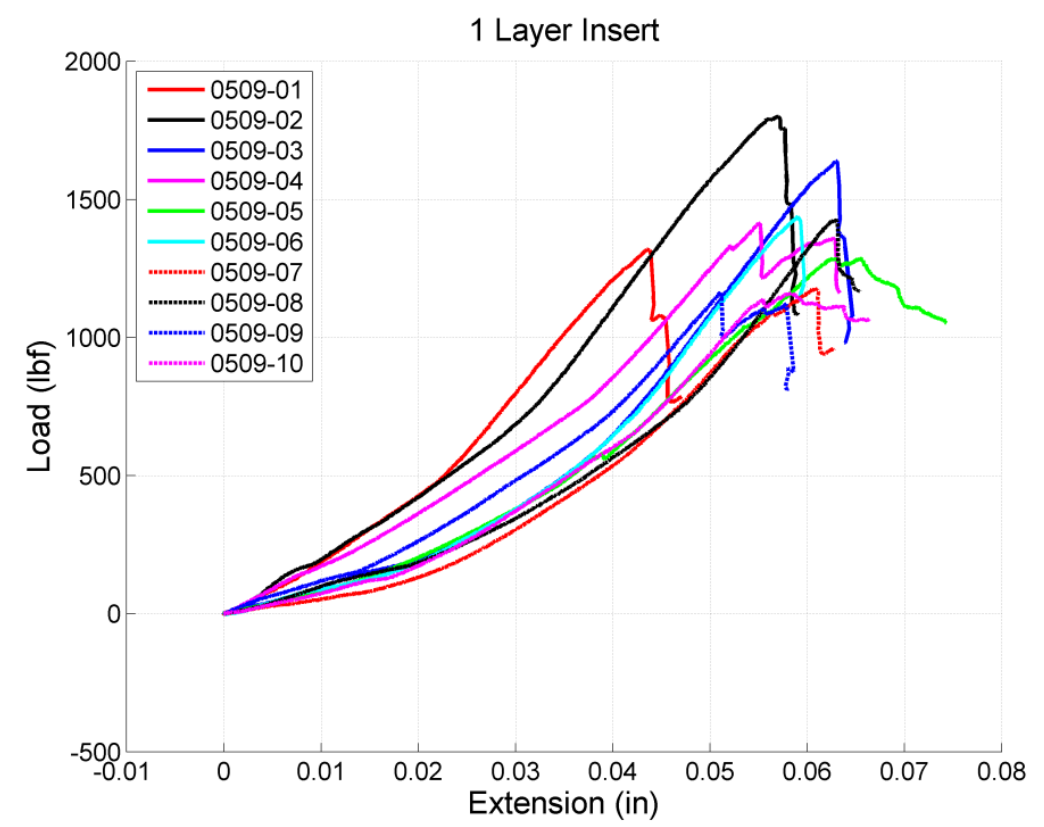

Figure 84: Load/Extension for DAD 1 Layer

\subsection{DAD 2 Layer Results}

The next static testing was the DAD 2 layer group; this can be seen in Figure 85. The test group had an increase of 55.3\% in ultimate load and an increase of 65.6\% in Elastic Modulus compared to the control group; this can be seen in Table 21. The addition of another layer for the DAD didn’t have a drastic increase compared to the DAD 1 Layer group. The test group had an increase of $13.5 \%$ in ultimate load and an increase of $6.2 \%$ in Elastic Modulus compared to the previous testing. This shows that adding an extra layer doesn't have that much of a big benefit as before. The test results show that the ultimate load had an average of 1,570 lbs and Elastic Modulus of 74,809 psi. 


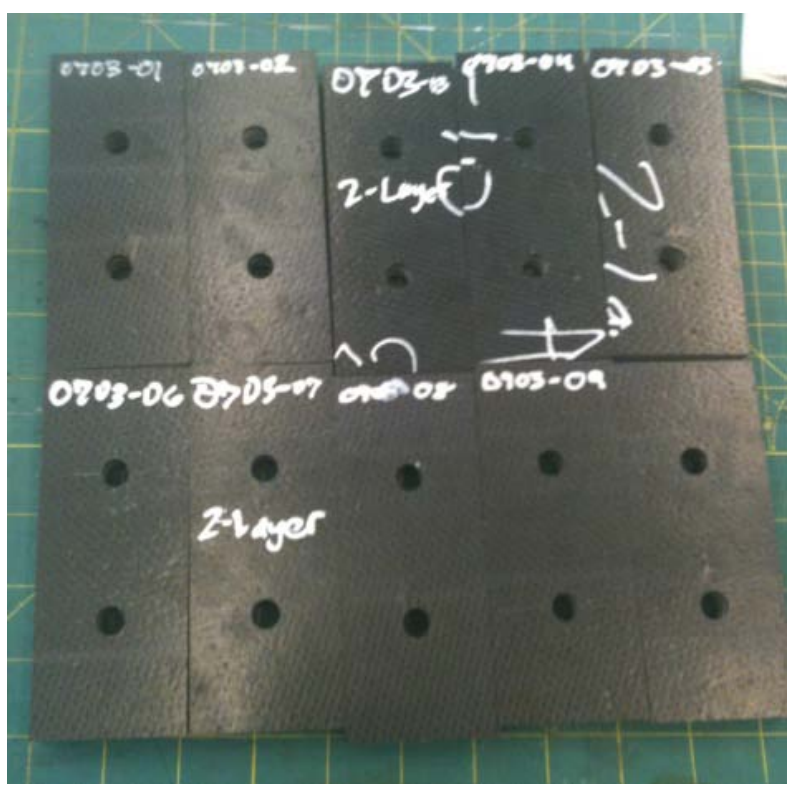

Figure 85: Test Specimens for DAD 2 Layer

Table 21: Test Results for DAD 2 Layer

\begin{tabular}{|c|c|c|c|c|c|c|}
\hline Test \# & $\begin{array}{c}\text { Test } \\
\text { Sample }\end{array}$ & $\begin{array}{c}\text { Ult } \\
\text { Load }\end{array}$ & Y Load & Extension & $\mathrm{K}$ & $\mathrm{E}$ \\
\hline 1 & 0703-01 & 1439 & 1439 & 0.0513 & 37869 & 64757 \\
\hline 2 & 0703-02 & 1776 & 1776 & 0.0642 & 39875 & 69573 \\
\hline 3 & 0703-03 & 1594 & 1594 & 0.0639 & 42256 & 73765 \\
\hline 4 & 0703-04 & 1609 & 1609 & 0.0619 & 41380 & 72054 \\
\hline 5 & 0703-05 & 1586 & 1586 & 0.0637 & 44010 & 75933 \\
\hline 6 & 0703-06 & 1601 & 1601 & 0.0633 & 51148 & 87963 \\
\hline 7 & 0703-07 & 1580 & 1580 & 0.0656 & 40500 & 68983 \\
\hline 8 & 0703-08 & 1460 & 1460 & 0.0528 & 45172 & 78463 \\
\hline 9 & 0703-09 & 1460 & 1460 & 0.0528 & 45172 & 78463 \\
\hline 10 & 0703-10 & 1591 & 1591 & 0.0612 & 45243 & 78141 \\
\hline \multicolumn{2}{|c|}{ Average } & 1570 & 1570 & 0.0601 & 43263 & 74809 \\
\hline \multicolumn{2}{|c|}{$\begin{array}{c}\text { Std Dev W/ } \\
\text { Outliers }\end{array}$} & $4.18 \%$ & $6.28 \%$ & $9.17 \%$ & $8.67 \%$ & $8.72 \%$ \\
\hline
\end{tabular}

The standard deviation for the test group shows that there are no outliers, having all ten specimens within one standard deviation from the mean; this can be seen in Figure 86. The specimens had a standard deviation of $4.18 \%$ from the mean for the ultimate load and a standard 
deviation of $8.72 \%$ from the mean for the Elastic Modulus. It shows that six of the ten testing specimens lie on the average, showing a very consistent test results for the case.

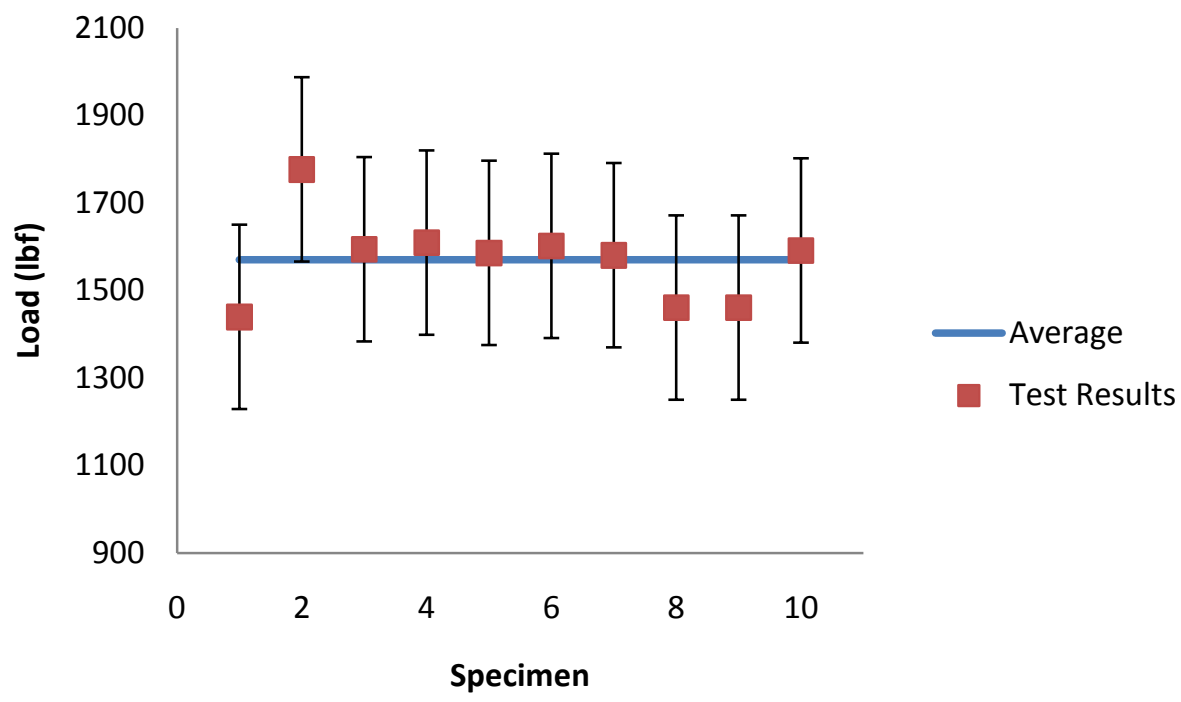

Figure 86: Standard Deviation for DAD 2 Layer

The load/extension curves for the case had consistent results; this can be seen in Figure 87. The trend for the group was that the specimen rises and then drops and continues dropping. The testing was stopped when the specimens started rising again. This was due to the fastener moving along the hole moving the face sheet with it. The test group had the same failure of the DAD "pops out" of the panel. The figure shows that the curves have similar slopes, showing that the Elastic Modulus and stiffness for the test group were similar to one another. The specimens also failed near one another around an extension of 0.06 in. 


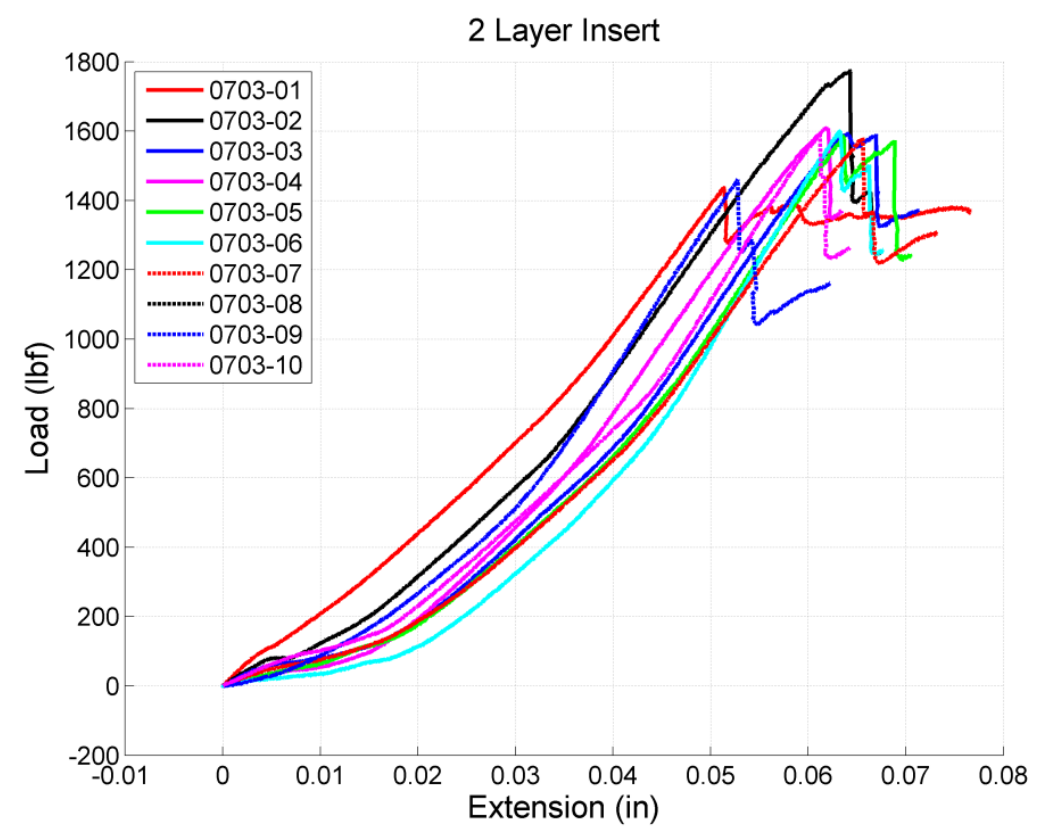

Figure 87: Load/Extension for DAD 2 Layer

\subsection{DAD 3 Layer Results}

The next static testing was the DAD 3 layer group; this can be seen in Figure 88. The test group had an increase of $63.5 \%$ in ultimate load and an increase of $31.4 \%$ in Elastic Modulus compared to the control group; this can be seen in Table 22. The results like the previous test group, show the increase of thickness in the DAD had a huge increase compared to the control group. Compared to the previous DAD layer, the test group had a minimal increase in ultimate load but a decrease in Elastic Modulus. The test group had an increase in 5.2\% for the ultimate load compared to the DAD 2 Layer and a decrease of 20.6\% in Elastic Modulus. The decrease in Elastic Modulus compared to the DAD 2 Layer could be due to the defects in manufacturing of the plate. The Elastic Modulus can also be due to how the value was calculated from the slopes of the curve; this will be discussed later in the section. The test results show that the ultimate load had an average of 1,652 lbs and Elastic Modulus of 59,340 psi. 


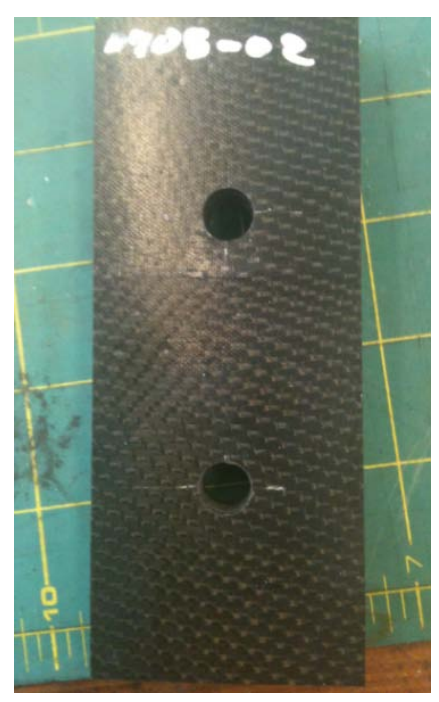

Figure 88: Test Specimen for DAD 3 Layer

Table 22: Test Results for DAD 3 Layer

\begin{tabular}{|c|c|c|c|c|c|c|}
\hline Test \# & Test Sample & $\begin{array}{c}\text { Ult } \\
\text { Load }\end{array}$ & Y Load & Extension & $\mathrm{K}$ & $\mathrm{E}$ \\
\hline 1 & 0705-01 & 1818.7 & 1818.74 & 0.0663 & 39190 & 65342 \\
\hline 2 & 0705-02 & 1717.3 & 1475.15 & 0.0632 & 32485 & 55910 \\
\hline 3 & 0705-03 & 1547.7 & 1547.74 & 0.0539 & 40759 & 69382 \\
\hline 4 & 0705-04 & 1562.1 & 1322.31 & 0.0633 & 24797 & 42383 \\
\hline 5 & 0705-05 & 1536.5 & 1536.47 & 0.0594 & 40107 & 69100 \\
\hline 6 & 0705-06 & 1776.1 & 1776.13 & 0.0564 & 39211 & 67340 \\
\hline 7 & 0705-07 & 1580.0 & 1395.61 & 0.0561 & 32538 & 55503 \\
\hline 8 & 0705-08 & 1379.6 & 1300.23 & 0.0614 & 24773 & 42238 \\
\hline 9 & 0705-09 & 1793.6 & 1611.58 & 0.0569 & 37985 & 63286 \\
\hline 10 & 0705-10 & 1807.8 & 1761.94 & 0.0606 & 36917 & 62913 \\
\hline \multicolumn{2}{|c|}{ Average Load } & 1651.93 & 1554.59 & 0.0597 & 34876 & 59340 \\
\hline \multicolumn{2}{|c|}{ Std Dev W/ Outliers } & $8.80 \%$ & $12.05 \%$ & $6.55 \%$ & $17.31 \%$ & $17.14 \%$ \\
\hline
\end{tabular}

The standard deviation for the test group shows that there was only one outlier; this can be seen in Figure 89. The eighth test specimen was the outlier of the group by being lower than one standard deviation from the mean. The specimens had a standard deviation of $8.80 \%$ from the mean for the ultimate load and a standard deviation of $17.14 \%$ from the mean for the Elastic Modulus. Half of the test specimens were above and below the average of the testing mean. The 
figure shows the outlier with an X marker and the specimens within one standard deviation have a square marker.

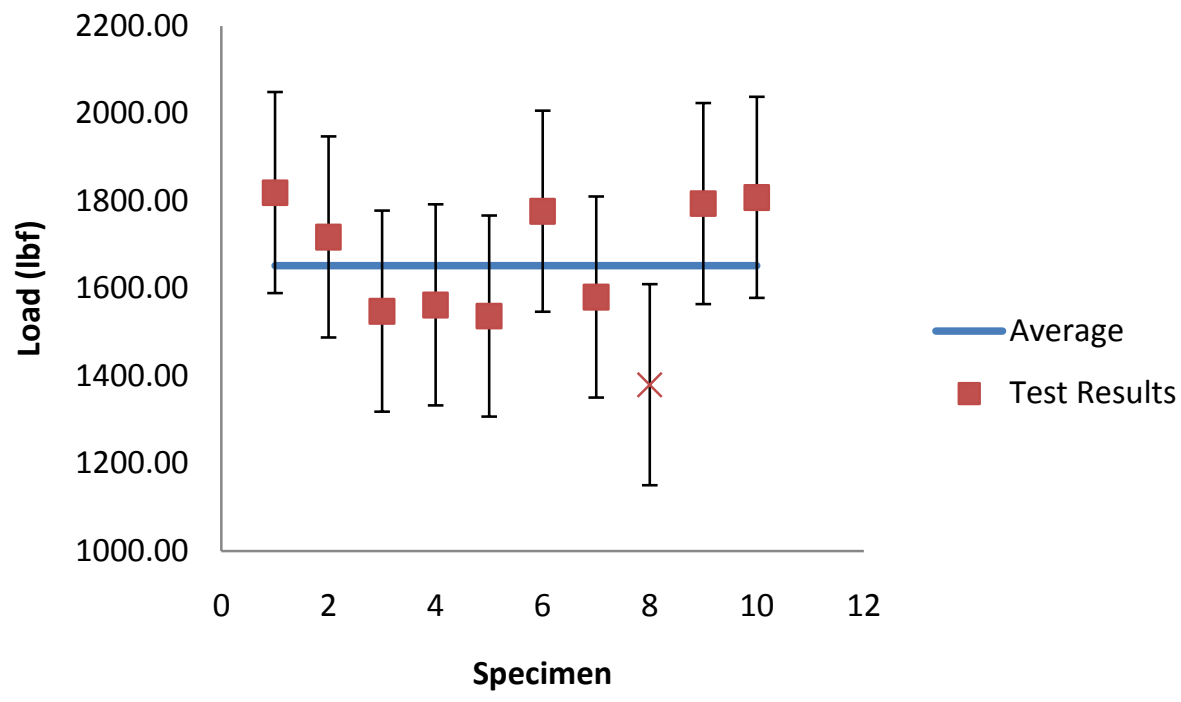

Figure 89: Standard Deviation for DAD 3 Layer

The load/extension curves for the case had consistent results; this can be seen in Figure 90. The trend for the group was that the specimen rises and then drops and levels out. The test group had the same failure of the DAD “pops out” of the panel. The figure shows that the test specimens had two different slopes. The first slope rose very erratically between the test specimens, having steep and leveled slopes. The second slope, which starts around an extension of 0.02 in, was pretty consistent among the test specimens. This was where the Elastic Modulus of the test specimens was calculated. The specimens failed between an extension of 0.05 in and 0.06 in. 


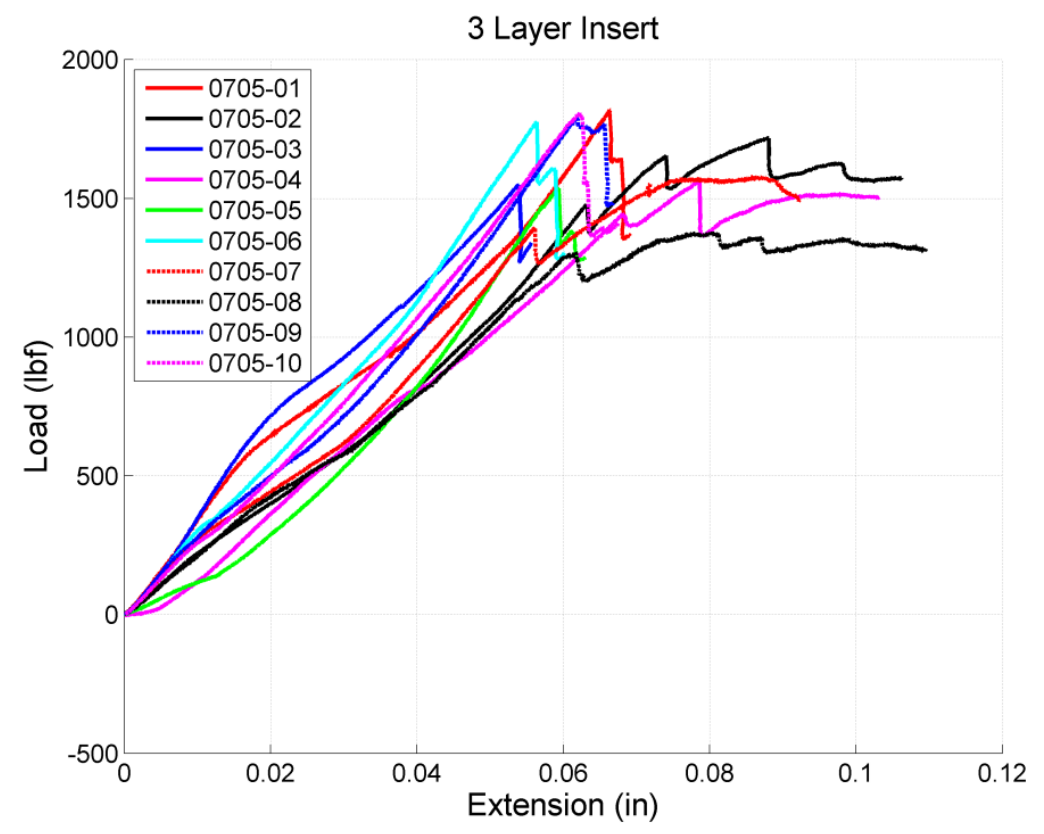

Figure 90: Load/Extension for DAD 3 Layer

\subsection{DAD 4 Layer Results}

The next static testing was the DAD 4 layer group; this can be seen in Figure 91. The test group had an increase of $84.2 \%$ in ultimate load and an increase of 91.6\% in Elastic Modulus compared to the control group; this can be seen in Table 23. The test group had an increase of $13.4 \%$ in ultimate load and an increase of $45.9 \%$ in Elastic Modulus compared to the previous testing. This test case shows that the DAD 3 Layer test group was an outlier for the Elastic Modulus. The trend so far for the Elastic Modulus was that it rose in a linear trend and that the drop in the value from the last test group could be on the low end of its value, meaning that it could be within a statistical average to the mean. The test results show that the ultimate load had an average of 1,860 lbs and Elastic Modulus of 86,568 psi. 


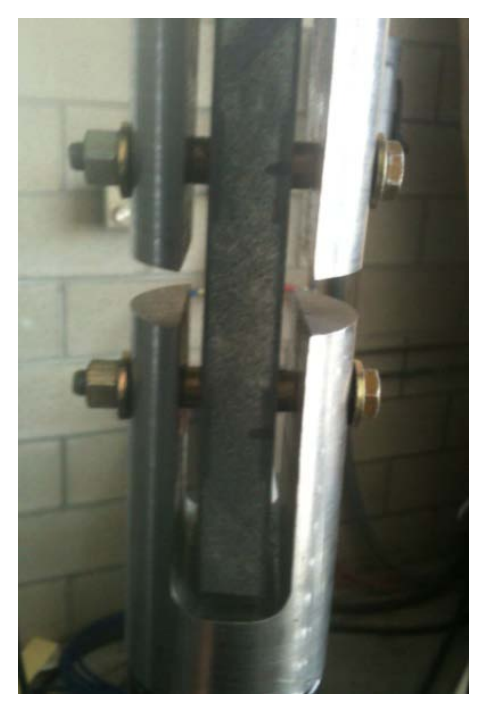

Figure 91: Test Specimen for DAD 4 Layer

Table 23: Test Results for DAD 4 Layer

\begin{tabular}{|c|c|c|c|c|c|c|}
\hline Test \# & $\begin{array}{c}\text { Test } \\
\text { Sample }\end{array}$ & Load & Y Load & Extension & $\mathrm{K}$ & $\mathrm{E}$ \\
\hline 1 & $0512-01$ & 1808.7 & 1808.75 & 0.0418 & 50397 & 85114 \\
\hline 2 & $0512-02$ & 1624.8 & 1079.66 & 0.0367 & 44292 & 73129 \\
\hline 3 & $0512-03$ & 1802.1 & 1780.23 & 0.0537 & 57695 & 98389 \\
\hline 4 & $0512-04$ & 1945.2 & 1456.18 & 0.0520 & 50061 & 85523 \\
\hline 5 & $0512-05$ & 1993.9 & 1856.16 & 0.0532 & 56687 & 99985 \\
\hline 6 & $0512-06$ & 1924.1 & 1623.54 & 0.0604 & 55125 & 95393 \\
\hline 7 & $0512-07$ & 1922.7 & 1921.48 & 0.0622 & 48746 & 83025 \\
\hline 8 & $0512-08$ & 1763.4 & 1465.94 & 0.0491 & 50823 & 88556 \\
\hline 9 & $0512-09$ & 1812.1 & 829.08 & 0.0446 & 38980 & 65954 \\
\hline 10 & $0512-10$ & 2006.7 & 1639.49 & 0.0530 & 53557 & 90617 \\
\hline \multicolumn{2}{|c|}{ Average Load } & 1860.374 & 1546.05 & 0.0507 & 50636 & 86568 \\
\hline \multicolumn{2}{|c|}{$\begin{array}{c}\text { Std Dev W/ } \\
\text { Outliers }\end{array}$} & $6.40 \%$ & $22.84 \%$ & $15.59 \%$ & $11.31 \%$ & $12.41 \%$ \\
\hline \multicolumn{2}{|c|}{$\begin{array}{c}\text { Std Dev W/O } \\
\text { Outliers }\end{array}$} & $4.51 \%$ & $10.59 \%$ & $11.82 \%$ & $6.43 \%$ & $7.15 \%$ \\
\hline
\end{tabular}

The standard deviation for the test group shows that there was only one outlier; this can be seen in Figure 92. The second, fifth, and tenth test specimens were the outliers of the group by being more than one standard deviation from the mean. The specimens had a standard deviation of $6.40 \%$ from the mean for the ultimate load and a standard deviation of $12.41 \%$ from the mean 
for the Elastic Modulus. The figure shows the outlier with an X marker and the specimens within one standard deviation have a square marker.

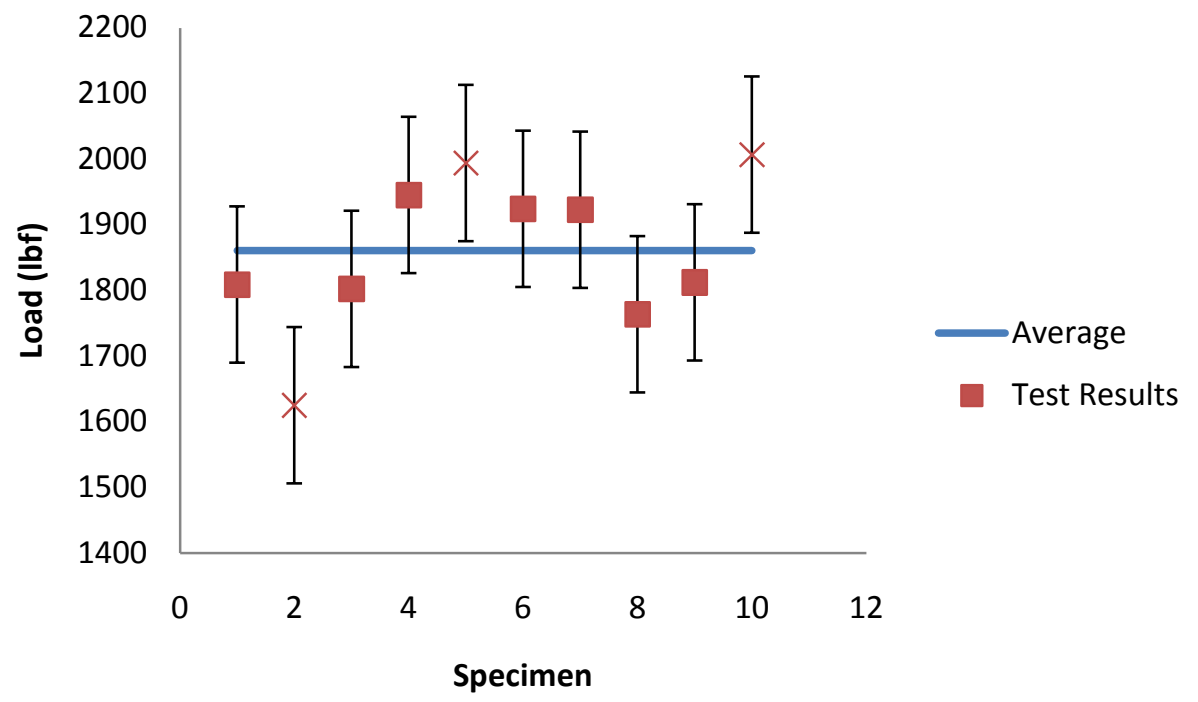

Figure 92: Standard Deviation for DAD 4 Layer

The load/extension curves for the case had consistent results; this can be seen in Figure 93. There are two trends for the group for the load/extension curve. The first trend was that the specimen rises and then drops and levels out. The second trend was that the specimen rose and then dropped severely. The test group had the same failure of the DAD "pops out" of the panel. The specimens failed between an extension of 0.04 in and 0.06 in. 


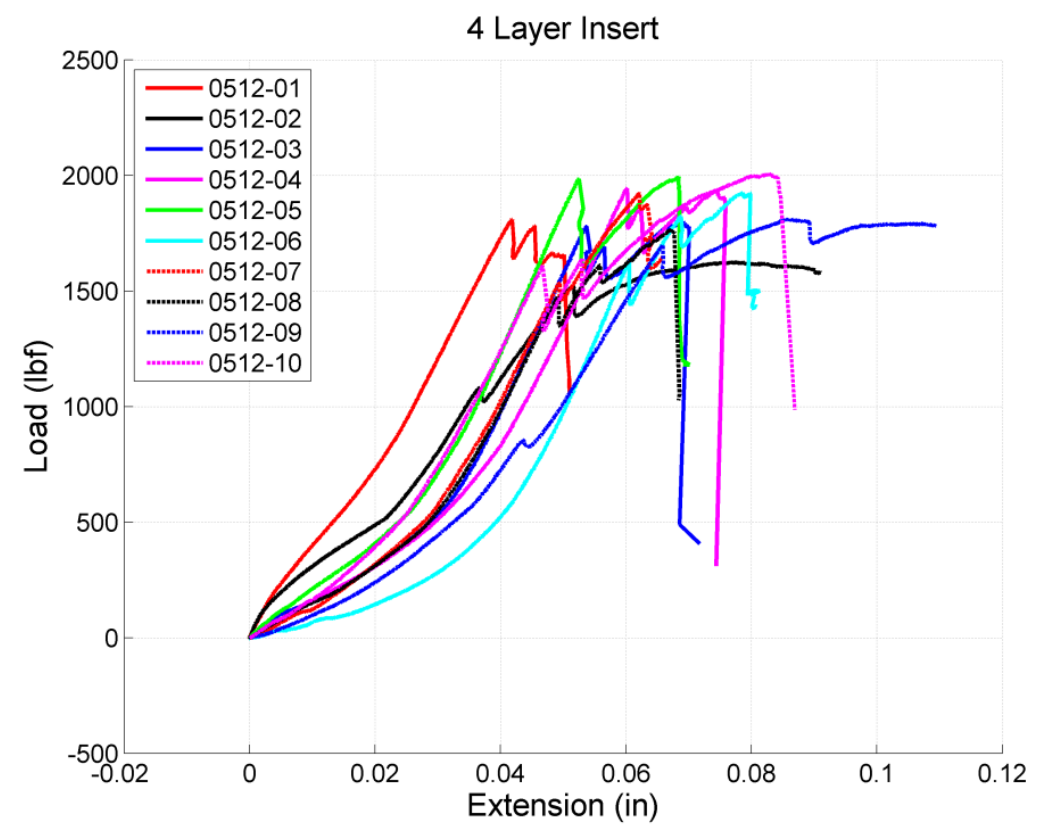

Figure 93: Load/Extension for DAD 4 Layer

\subsection{DAD 5 Layer Results}

The next static testing was the DAD 5 layer group; this can be seen in Figure 94. The test group had an increase of $113.7 \%$ in ultimate load and an increase of $128.1 \%$ in Elastic Modulus compared to the control group; this can be seen in Table 24. The results like the previous test group, showed the increase of thickness in the DAD had a huge increase compared to the control group. Compared to the previous DAD layer, the test group had a minimal increase in ultimate load and Elastic Modulus. The test group had an increase in $16.0 \%$ for the ultimate load compared to the DAD 4 Layer and an increase of 19.1\% in Elastic Modulus. The test results show that the ultimate load had an average of 2,158 lbs and Elastic Modulus of 103,062 psi. 


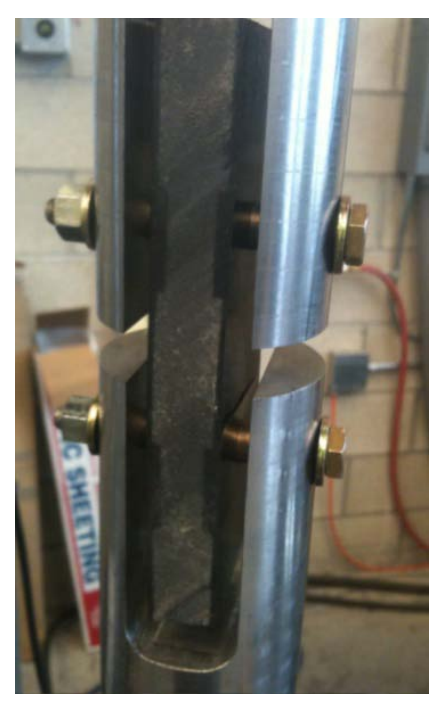

Figure 94: Test Specimen for DAD 5 Layer

Table 24: Test Results for DAD 5 Layer

\begin{tabular}{|c|c|c|c|c|c|c|}
\hline Test \# & $\begin{array}{c}\text { Test } \\
\text { Sample }\end{array}$ & Load & Y Load & Extension & K & E \\
\hline 1 & $0412-01$ & 2270.67 & 1901.41 & 0.0521 & 53514 & 95041 \\
\hline 2 & $0412-02$ & 2353.90 & 2199.08 & 0.0526 & 52659 & 94684 \\
\hline 3 & $0412-03$ & 2130.52 & 1899.39 & 0.0468 & 55758 & 101425 \\
\hline 4 & $0412-04$ & 1983.62 & 1864.49 & 0.0460 & 59329 & 109876 \\
\hline 5 & $0412-05$ & 2250.02 & 1839.48 & 0.0509 & 58486 & 101635 \\
\hline 6 & $0412-06$ & 2128.66 & 2128.66 & 0.0560 & 59513 & 102025 \\
\hline 7 & $0412-07$ & 1901.03 & 1758.48 & 0.0385 & 60416 & 105881 \\
\hline 8 & $0412-08$ & 2112.88 & 2112.88 & 0.0575 & 61617 & 107593 \\
\hline 9 & $0412-09$ & 2127.14 & 1715.65 & 0.0436 & 59447 & 106105 \\
\hline 10 & $0412-10$ & 2325.54 & 2242.42 & 0.0562 & 60672 & 106358 \\
\hline \multicolumn{2}{|c|}{ Average Load } & 2158.397 & 1966.19 & 0.0500 & 58141 & 103062 \\
\hline \multicolumn{2}{|c|}{$\begin{array}{c}\text { Std Dev W/ } \\
\text { Outliers }\end{array}$} & $6.71 \%$ & $9.58 \%$ & $12.32 \%$ & $5.31 \%$ & $4.96 \%$ \\
\hline \multicolumn{2}{|c|}{$\begin{array}{c}\text { Std Dev W/O } \\
\text { Outliers }\end{array}$} & $0.38 \%$ & $6.56 \%$ & $9.10 \%$ & $5.02 \%$ & $5.09 \%$ \\
\hline
\end{tabular}

The standard deviation for the test group shows that there was only one outlier; this can be seen in Figure 95. This test group had an interesting standard deviation for the load failure because half of the test specimens were outside of one standard deviation. The first, second, fourth, fifth, seventh, and tenth test specimens were outside of one standard deviation from the 
mean. The specimens that were within one standard deviation were all near below the test mean, having a standard deviation of $0.38 \%$ for those specimens. The specimens had a standard deviation of $6.71 \%$ from the mean for the ultimate load and a standard deviation of $4.96 \%$ from the mean for the Elastic Modulus. The figure shows the outlier with an X marker and the specimens within one standard deviation have a square marker.

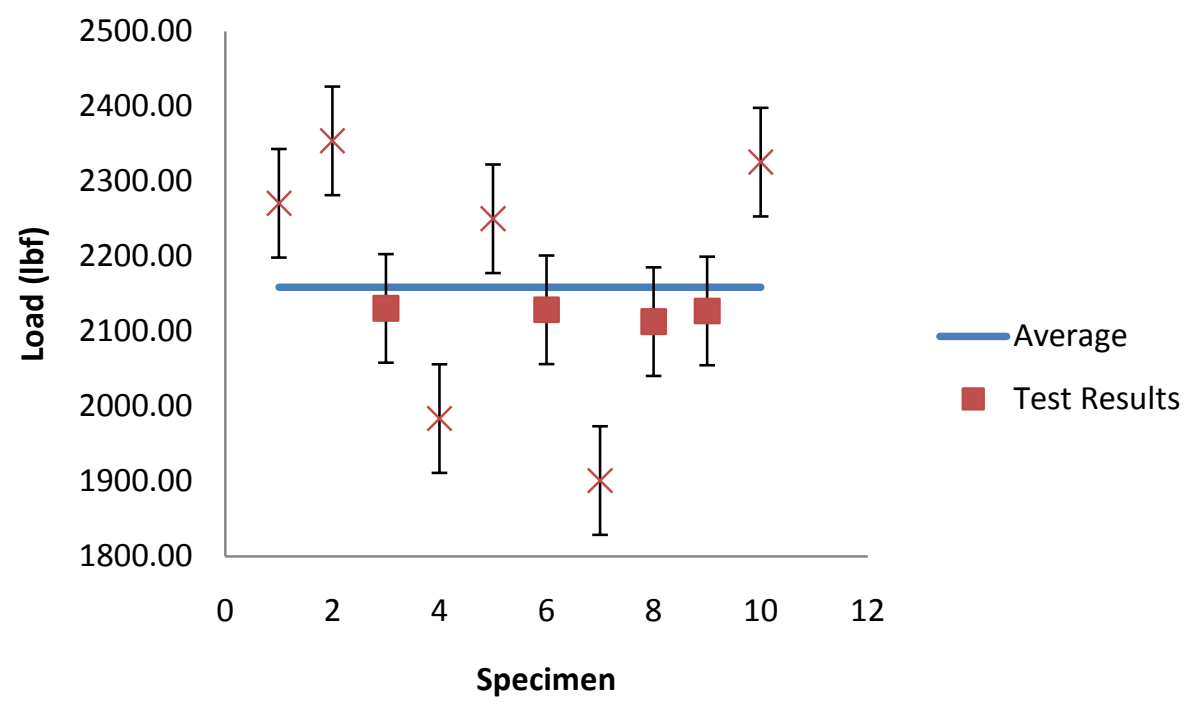

Figure 95: Standard Deviation for DAD 5 Layer

The load/extension curves for the case had consistent results; this can be seen in Figure 96. The trend for this test group was that the specimen rose and then dropped and then rose again, but the second drop has an equivalent load than the previous drop. The second rise and having an equivalent load could be due to the face sheet fracturing along the $\mathrm{DAD}$ for the composite sandwich panel. The test group had the same failure of the DAD "pops out” of the panel, but starts to differ from previous groups. The specimens also had buckling involved in the failure mode showing that the DADs were starting to dominate the thickness of the core. The specimens failed between an extension of 0.04 in and 0.06 in. 


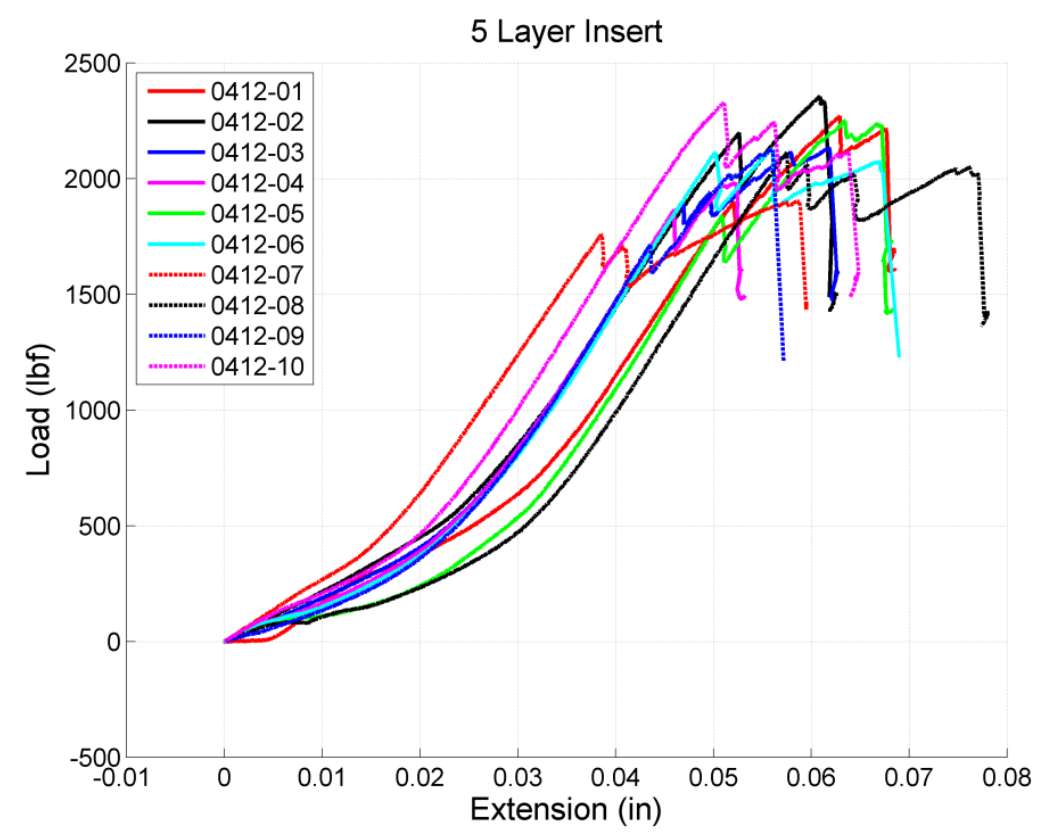

Figure 96: Load/Extension for DAD 5 Layer

\subsection{DAD 6 Layer Results}

The next static testing was the DAD 6 layer group; this can be seen in Figure 97. The test group had an increase of $102.1 \%$ in ultimate load and an increase of $112.4 \%$ in Elastic Modulus compared to the control group; this can be seen in Table 25. The results like the previous test group, the increase of thickness in the DAD had a huge increase compared to the control group but had no minimal increase in ultimate load and Elastic Modulus. The test group had a decrease in $1.85 \%$ for the ultimate load compared to the DAD 5 Layer and a decrease of $6.9 \%$ in Elastic Modulus. The test results show that the ultimate load had an average of 2,118 lbs and Elastic Modulus of 95,953 psi. 


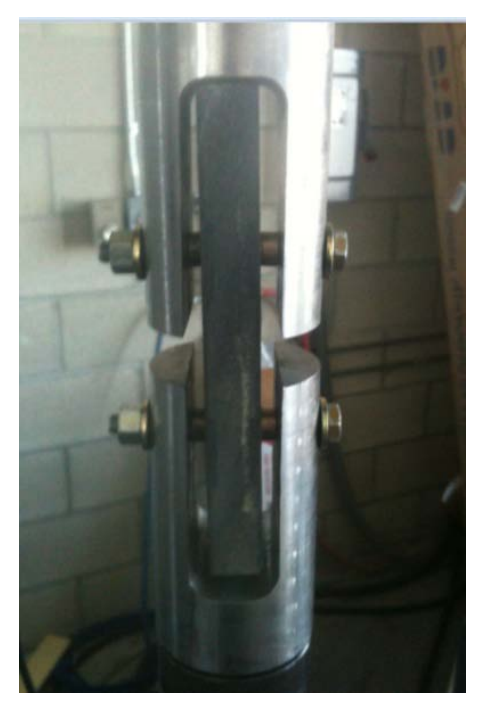

Figure 97: Test Specimen for DAD 6 Layer

Table 25: Test Results for DAD 6 Layer

\begin{tabular}{|c|c|c|c|c|c|c|}
\hline Test \# & $\begin{array}{c}\text { Test } \\
\text { Sample }\end{array}$ & Load & Y Load & Extension & K & E \\
\hline 1 & $0526-01$ & 2135.48 & 1502.14 & 0.0572 & 53831 & 92334 \\
\hline 2 & $0526-02$ & 2106.43 & 2044.58 & 0.0566 & 58365 & 95479 \\
\hline 3 & $0526-03$ & 1575.34 & 1440.01 & 0.0381 & 54259 & 93486 \\
\hline 4 & $0526-04$ & 1973.24 & 1720.83 & 0.0417 & 59992 & 100774 \\
\hline 5 & $0526-05$ & 2212.74 & 1755.72 & 0.0406 & 64575 & 107291 \\
\hline 6 & $0526-06$ & 2010.34 & 1802.57 & 0.0585 & 55864 & 93885 \\
\hline 7 & $0526-07$ & 2283.91 & 1767.25 & 0.0586 & 56655 & 97469 \\
\hline 8 & $0526-08$ & 2411.44 & 2182.96 & 0.0651 & 57372 & 95901 \\
\hline 9 & $0526-09$ & 2278.54 & 1610.55 & 0.0516 & 55051 & 87539 \\
\hline 10 & $0526-10$ & 2192.75 & 2063.26 & 0.0639 & 56854 & 95372 \\
\hline \multicolumn{2}{|c|}{ Average Load } & 2118.021 & 1788.99 & 0.0532 & 57282 & 95953 \\
\hline \multicolumn{2}{|c|}{$\begin{array}{r}\text { Std Dev W/ } \\
\text { Outliers }\end{array}$} & $10.92 \%$ & $13.67 \%$ & $18.40 \%$ & $5.53 \%$ & $5.48 \%$ \\
\hline \multicolumn{2}{|c|}{ Std Dev W/O } \\
Outliers & $4.58 \%$ & $15.19 \%$ & $22.72 \%$ & $7.06 \%$ & $5.90 \%$ \\
\hline
\end{tabular}

The standard deviation for the test group shows that there was only one outlier; this can be seen in Figure 98. This test group was similar to the last test group for the standard deviation for the load failure because half of the test specimens were outside of one standard deviation. The third, fourth, seventh, eight, and ninth test specimens were outside of one standard deviation from the mean. The specimens had a standard deviation of $10.92 \%$ from the mean for the ultimate load 
and a standard deviation of $5.48 \%$ from the mean for the Elastic Modulus. The figure shows the outlier with an X marker and the specimens within one standard deviation had a square marker.

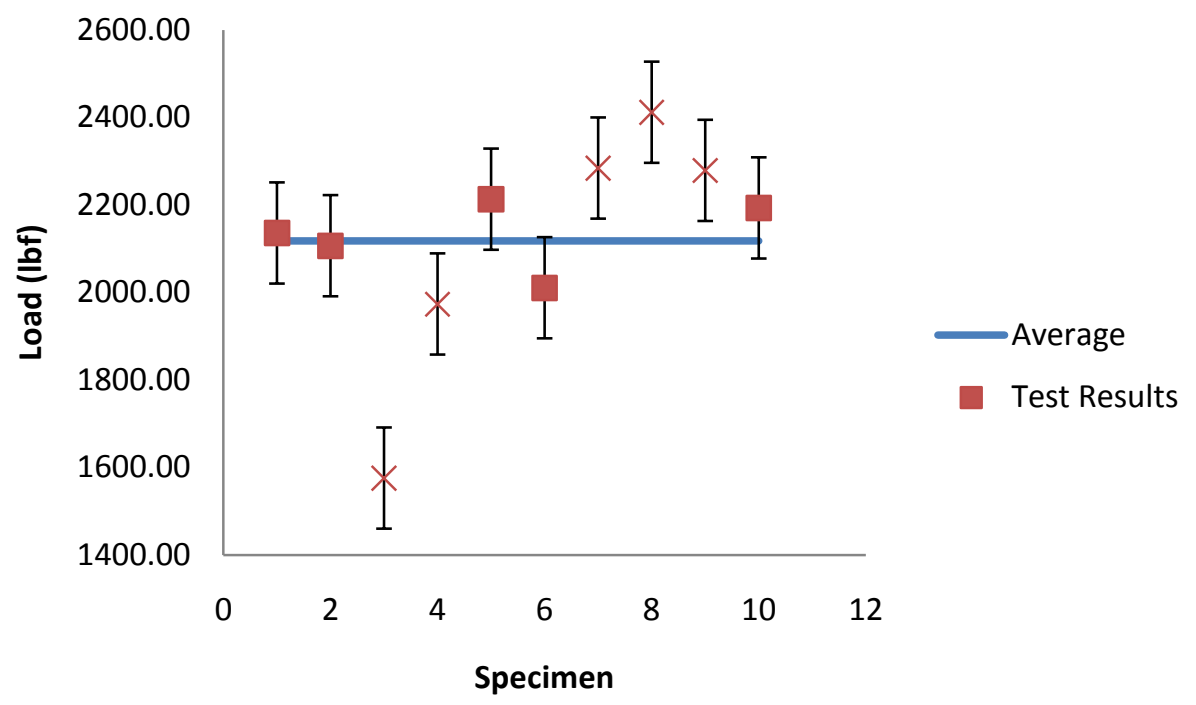

Figure 98: Standard Deviation for DAD 6 Layer

The load/extension curves for the case had consistent results; this can be seen in Figure 99. There we two trends for the group for the load/extension curve. The first trend was that the specimen rose and then dropped and leveled out and then severely dropped. The second trend was that the specimen rose and then dropped. These load/extension curves had an ideal curve by rising and then dropping and then rising again as a parabolic curve and then failing at a certain point. The test group had the same failure of the DAD "pops out” and buckling of the panel. The buckling of the panels could be a reason for the erratic curves from the specimen. It can also explain the number of test specimens that were at least one standard deviation from the mean. The specimens failed between an extension of 0.03 in and 0.06 in. 


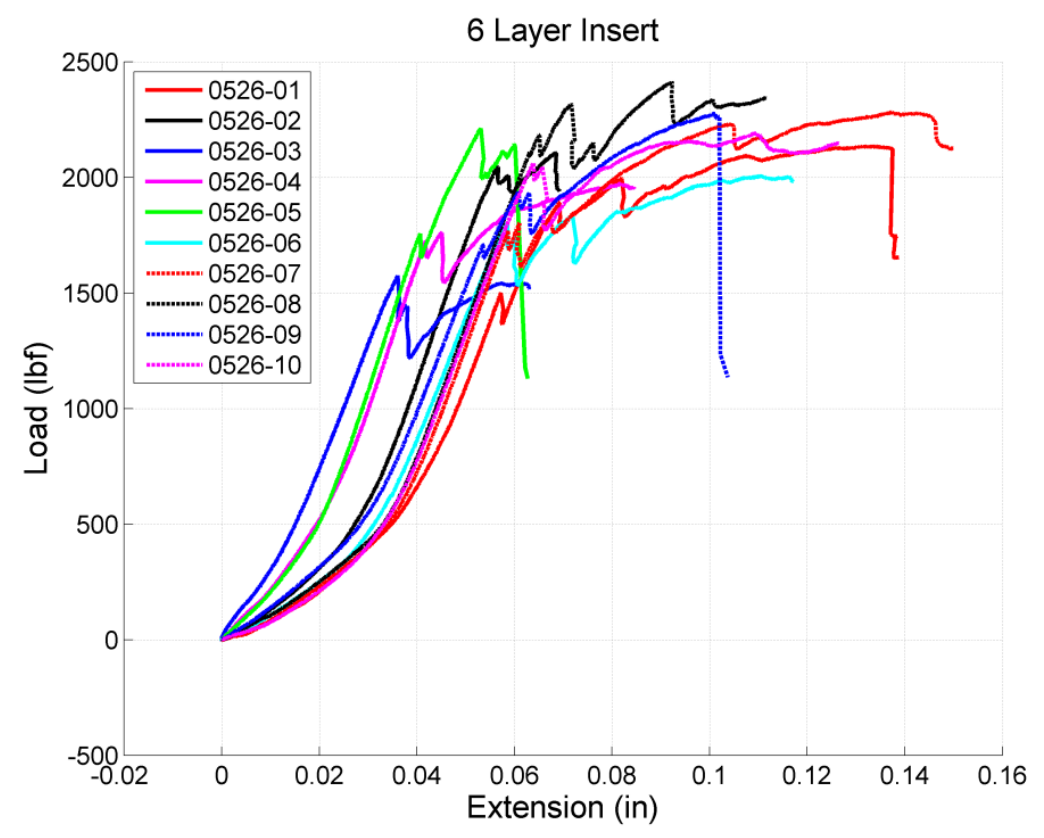

Figure 99: Load/Extension for DAD 6 Layer

\subsection{DAD 7 Layer Results}

The next static testing was the DAD 7 layer group; this can be seen in Figure 100. The test group had an increase of $99.7 \%$ in ultimate load and an increase of $46.5 \%$ in Elastic Modulus compared to the control group; this can be seen in Table 26. The results like the previous test group, showed the increase of thickness in the DAD had a huge increase compared to the control group but had no minimal increase in ultimate load and Elastic Modulus. The test group had a decrease in $4.77 \%$ for the ultimate load compared to the DAD 6 Layer and a decrease of $31.1 \%$ in Elastic Modulus. The test results had a huge decrease in the Elastic Modulus from the previous test group. The test results show that the ultimate load had an average of 2,017 lbs and Elastic Modulus of 66,196 psi. 


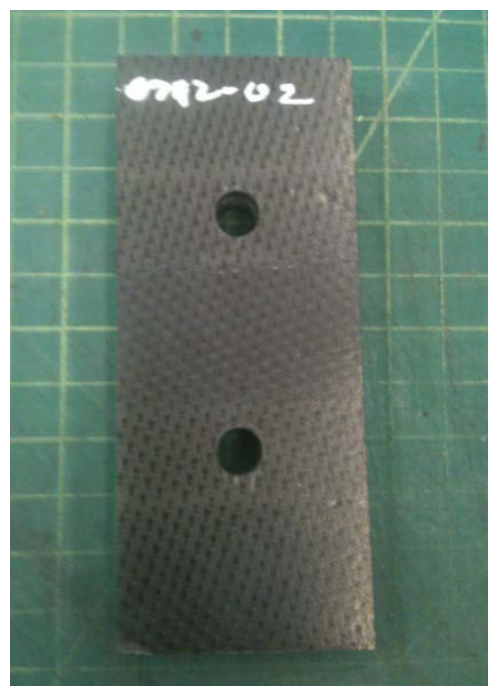

Figure 100: Test Specimens for DAD 7 Layer

Table 26: Test Results for DAD 7 Layer

\begin{tabular}{|c|c|c|c|c|c|c|}
\hline Test \# & $\begin{array}{c}\text { Test } \\
\text { Sample }\end{array}$ & Load & Y Load & Extension & K & $\mathrm{E}$ \\
\hline 1 & 0712-01 & 1928.88 & 1118.96 & 0.0423 & 34048 & 59718 \\
\hline 2 & 0712-02 & 1871.71 & 1400.42 & 0.0709 & 34230 & 58322 \\
\hline 3 & 0712-03 & 1777.33 & 1600.37 & 0.0824 & 31080 & 54459 \\
\hline 4 & 0712-04 & 1864.06 & 1689.12 & 0.0592 & 46476 & 79597 \\
\hline 5 & 0712-05 & 2435.16 & 1189.97 & 0.0598 & 39175 & 66041 \\
\hline 6 & 0712-06 & 1917.45 & 934.91 & 0.0549 & 48838 & 83348 \\
\hline 7 & 0712-07 & 2110.02 & 997.48 & 0.0428 & 39602 & 67935 \\
\hline 8 & 0712-08 & 1969.70 & 842.30 & 0.0386 & 38501 & 65067 \\
\hline 9 & 0712-09 & 2121.01 & 1001.23 & 0.0637 & 32852 & 56939 \\
\hline 10 & 0712-10 & 2180.44 & 2037.53 & 0.0760 & 41735 & 70532 \\
\hline \multicolumn{2}{|c|}{ Average Load } & 2017.575 & 1281.23 & 0.0591 & 38654 & 66196 \\
\hline \multicolumn{2}{|c|}{$\begin{array}{c}\text { Std Dev W/ } \\
\text { Outliers }\end{array}$} & $9.67 \%$ & $30.35 \%$ & $25.09 \%$ & $15.12 \%$ & $14.46 \%$ \\
\hline
\end{tabular}

The standard deviation for the test group shows that there was only one outlier; this can be seen in Figure 101. This test group was similar to the last test group for the standard deviation for the load failure because half of the test specimens are outside of one standard deviation. The second, third, fourth, fifth, and tenth test specimens were outside of one standard deviation from the mean. The specimens had a standard deviation of $9.67 \%$ from the mean for the ultimate load 
and a standard deviation of $14.46 \%$ from the mean for the Elastic Modulus. The figure shows the outlier with an $\mathrm{X}$ marker and the specimens within one standard deviation have a square marker.

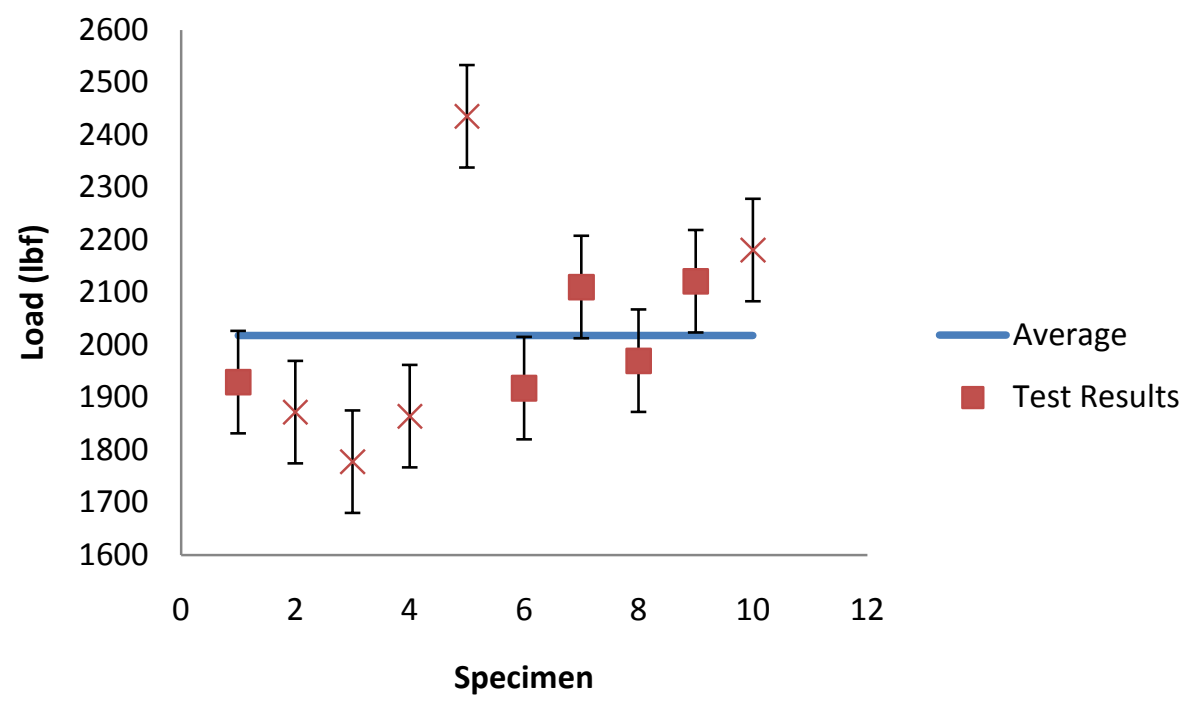

Figure 101: Standard Deviation for DAD 7 Layer

The load/extension curves for the case had consistent results; this can be seen in Figure 102. There were two trends for the group for the load/extension curve. The first trend was that the specimen rose and then dropped and rose again and repeated until failure. Some of the test specimens had three or four drops and rises. The second trend was that the specimen rose and then leveled out. These load/extension curves had an ideal curve by rising and then dropping and then rising again and a parabolic curve and then failing at a certain point. The test group had the same failure of the DAD "pops out" of the panel and buckling of the panel. The buckling of the panels could be a reason the erratic curves from the specimen. It can also explain the number of test specimens that were at least one standard deviation from the mean. The specimens failed between an extension of 0.03 in and 0.07 in. This test group had the largest variance in extension for the first drop. This could be due to how the thickness of the DADs affected the overall thickness of the composite sandwich panel. 


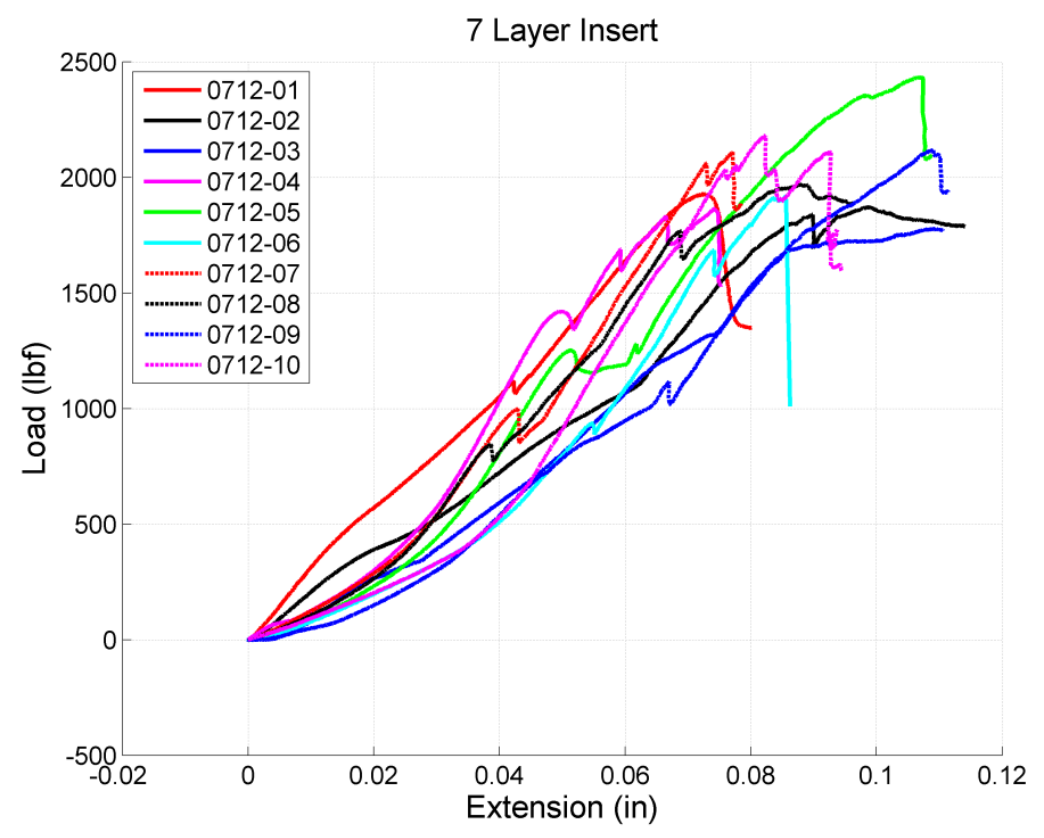

Figure 102: Load/Extension for DAD 7 Layer

\subsection{DAD Static Trend}

With enough DAD thickness tested, a trend can be observed for the ultimate failure load for the static loading; this can be seen in Table 27. The test results showed that the trend for the ultimate load rose and can have a linear, polynomial, or logarithmic trend as the thickness of the DADs rises. This showed that regardless of whatever the thickness of the DAD, the extension for the specimen stayed the same for any thickness. The results showed that the ultimate load for the DAD thickness rose and then dropped after the DAD 5 Layer test group. The test group could be the tipping point for the DADs by having the most effectiveness without adding any more excess weight. This could be due to the buckling seen from the specimens that would cause the tipping point of the trend. This also shows that the DAD thickness started affecting the overall thickness of the composite sandwich panel. As the thickness of the DADs increased, it started to dominate by taking up most of the core around the fastener. The yield extension was varied for the test results, but was all within a range of 0.05 in to $0.601 \mathrm{in}$. 
Table 27: DAD Trend Test Results

\begin{tabular}{|c|c|c|c|c|c|}
\hline $\begin{array}{c}\text { DAD } \\
\text { Thickness }\end{array}$ & $\begin{array}{c}\text { Ult } \\
\text { Load }\end{array}$ & Y Load & Extension & K & E \\
\hline 0 & 1010 & 940 & 0.0544 & 25285 & 45174 \\
\hline 1 & 1383 & 1341 & 0.0556 & 40945 & 70468 \\
\hline 2 & 1570 & 1570 & 0.0601 & 43263 & 74809 \\
\hline 3 & 1652 & 1555 & 0.0597 & 34876 & 59340 \\
\hline 4 & 1860 & 1546 & 0.0507 & 50636 & 86568 \\
\hline 5 & 2158 & 1966 & 0.0500 & 58141 & 103062 \\
\hline 6 & 2118 & 1789 & 0.0532 & 57282 & 95953 \\
\hline 7 & 2018 & 1281 & 0.0591 & 38654 & 66196 \\
\hline
\end{tabular}

The ultimate load DAD thickness trend can be seen in Figure 103. The trend showed a linear and parabolic trend line with its coefficient of determination, $\mathrm{R}^{2}$. The trend showed that a linear trend had a value of 0.8702 while the parabolic trend line had a value of 0.9587 . The parabolic trend captured the trend pretty well by taking into account the drop in loading after the DAD 5 Layer. It also showed that each test group, except the DAD 5 layer, was within one standard deviation of the trend line. The trend could still prove that if the DAD 5 layer test group was retested, the results could drop and have the save loading as the DAD 6 and 7 layers. Another test case had to be tested. DAD 8 Layer might need to be investigated to see if the trend becomes logarithmic. 


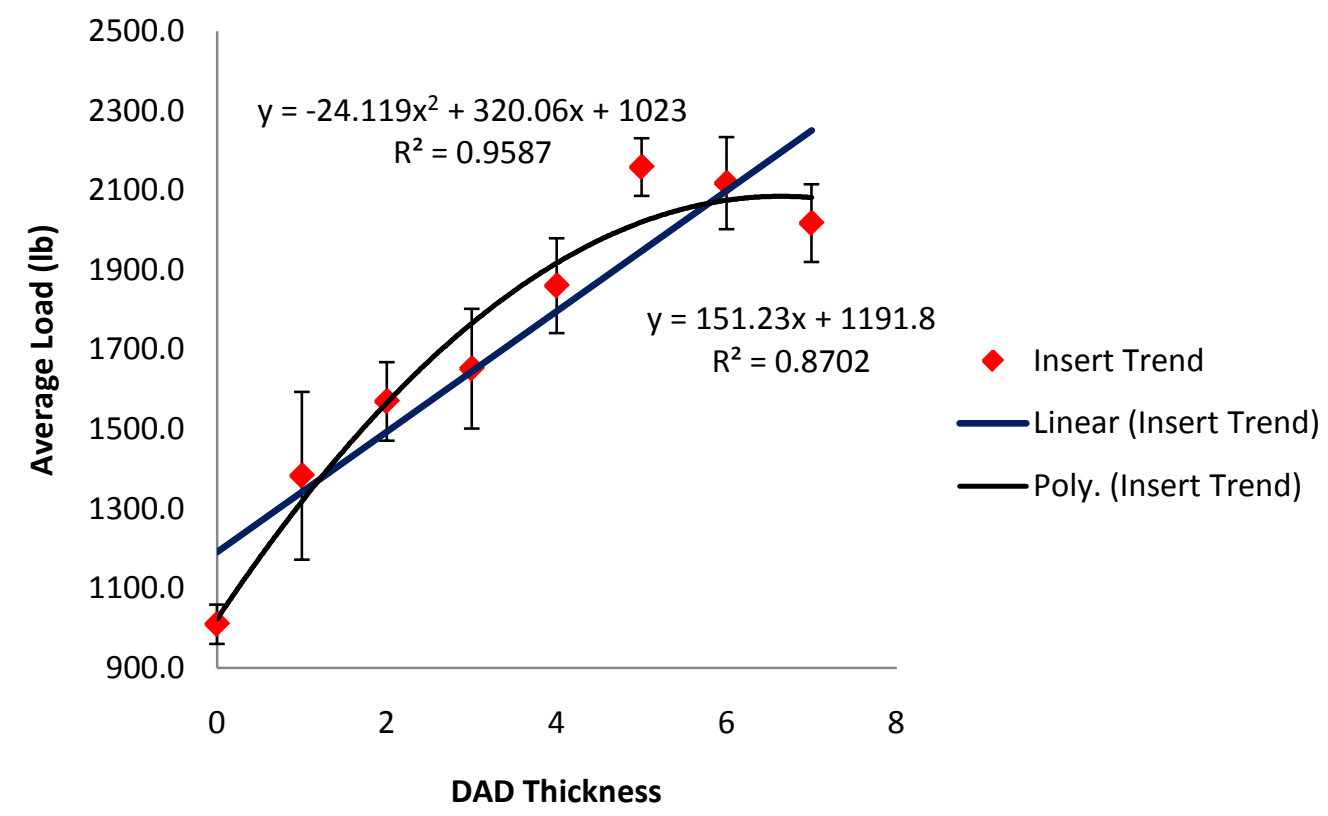

Figure 103: Ultimate Load DAD thickness trend

The Elastic Modulus DAD thickness trend can be seen in Figure 104. The trend shows that there was no trend for the Elastic Modulus for the DAD thickness trend because its polynomial and linear coefficient of determination was too low. The trend shows that the DAD 3 Layer Elastic Modulus was an outlier to the trend. If that data point was omitted, the parabolic trend line increased to 0.8392 from 0.5739 . It showed that the DAD 3 Layer test group had to be redone to determine a more accurate Elastic Modulus trend for the static testing. This would show that the Elastic Modulus would follow an identical trend to the Ultimate Failure Load of the specimens. 


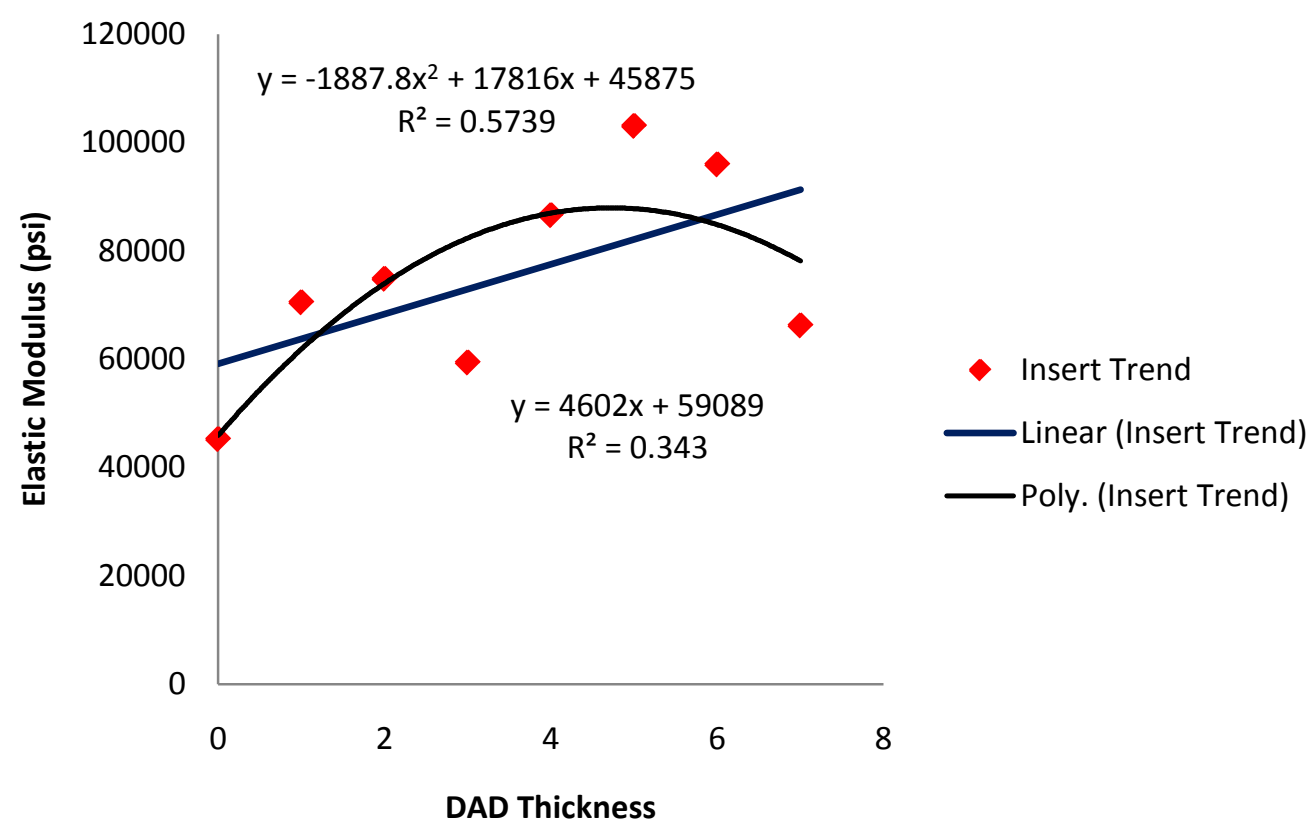

Figure 104: Elastic Modulus DAD thickness Trend

\subsection{Fatigue Control Group Results}

The fatigue control group results showed a broad range of results for the fatigue cases tested. The average number of cycles for failure test results can be seen in Table 28. The results showed that as the fatigue amplitude of the testing decreased, the number of test cycles increased exponentially. The specimens for this test group greatly varied, this can be seen in Figure 105. The plot showed the fatigue amplitude versus the number of cycles for failure at that amplitude in a log scale for the number of cycles. The figure showed a vast range of test results by having a huge range between the numbers of failures for each amplitude group.

Table 28: Test Results for Fatigue Control Group

\begin{tabular}{|c|c|}
\hline Fatigue Amplitude & Average Number of Cycles to Failure \\
\hline 90 & 310 \\
\hline 85 & 1,999 \\
\hline 80 & 1,771 \\
\hline 75 & 5,529 \\
\hline 70 & 53,269 \\
\hline 65 & 500,000 \\
\hline
\end{tabular}




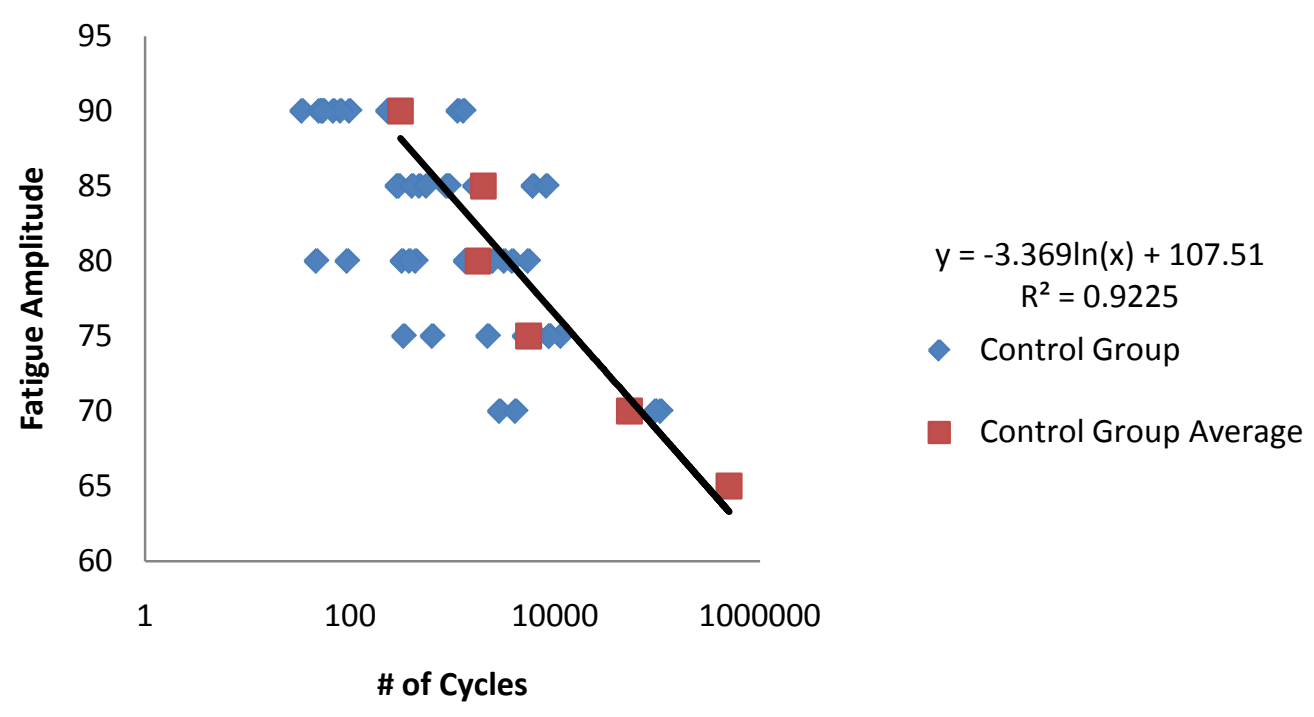

Figure 105: Control Group Trend

The standard deviation for each test group ranged from 56\% to 93\%. The huge standard deviation was due to the manufacturing defects of the test specimens. The purpose of fatigue testing was to investigate how the material fails from a constant loading and unloading of a specimen. Since specimens cannot be completely identical to one another, failure of a specimen was due to the defects of that specimen. Trends were more important to the testing by knowing the characteristics of failure for the specimen.

The results showed that specimens with many manufacturing defects tended to fail quicker. A lot of the test specimens were clumped to the left side of the chart, due to the defects of the specimens. Specimens that had an ideal failure result were on the right side of the test results. The results showed that the results were in the extremes of the material.

The averages of the specimens were calculated to be used for the trend line of the material. The trend line for the control group fatigue testing had a coefficient of determination of 0.9225, showing that a logarithmic trend was ideal for the material property. The test results in a Cartesian scale showed that the endurance limit of the material was between $65 \%$ to $70 \%$ fatigue amplitude of the yield stress; this can be seen in Figure 106. The trend showed that the number of 
failures levels out after amplitude of $70 \%$ was reached. This was helpful for designing a system with the use of the material.

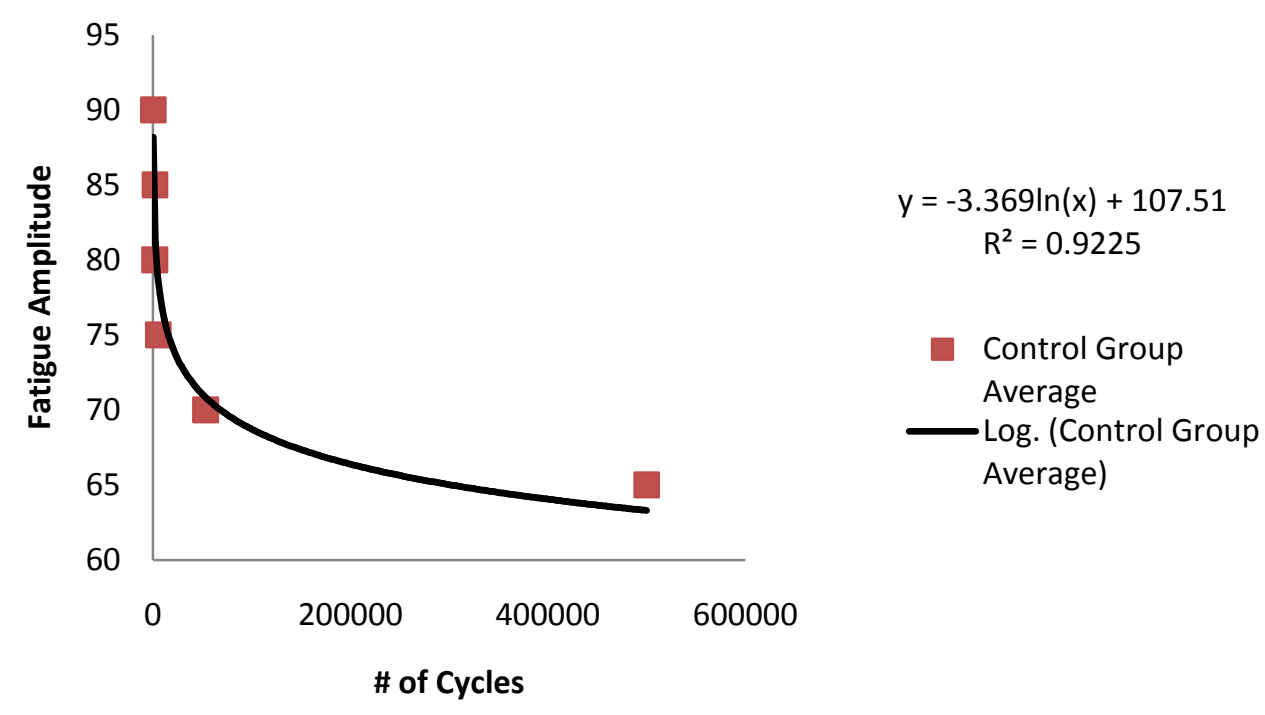

Figure 106: Control Group Average Results

\subsection{Fatigue DAD Results}

The fatigue DAD results showed a broad range of results for the fatigue cases tested. The average number of cycles for failure test results can be seen Table 29. The results showed that as the fatigue amplitude of the testing decreased, the number of test cycles increased exponentially. The specimens for this test group greatly varied, this can be seen in Figure 107. The plot shows the fatigue amplitude versus the number of cycles for failure at that amplitude in a log scale for the number of cycles. The figure shows a vast range of test results by having a huge range between the numbers of failures for each amplitude group.

Table 29: Test Results for Fatigue DAD Group

\begin{tabular}{|c|c|}
\hline Average Fatigue Amplitude & Average Number of Cycles to Failure \\
\hline 90 & 183 \\
\hline 85 & 1,525 \\
\hline 80 & 1,665 \\
\hline 75 & 7,311 \\
\hline 70 & 51,504 \\
\hline 65 & 872,560 \\
\hline
\end{tabular}




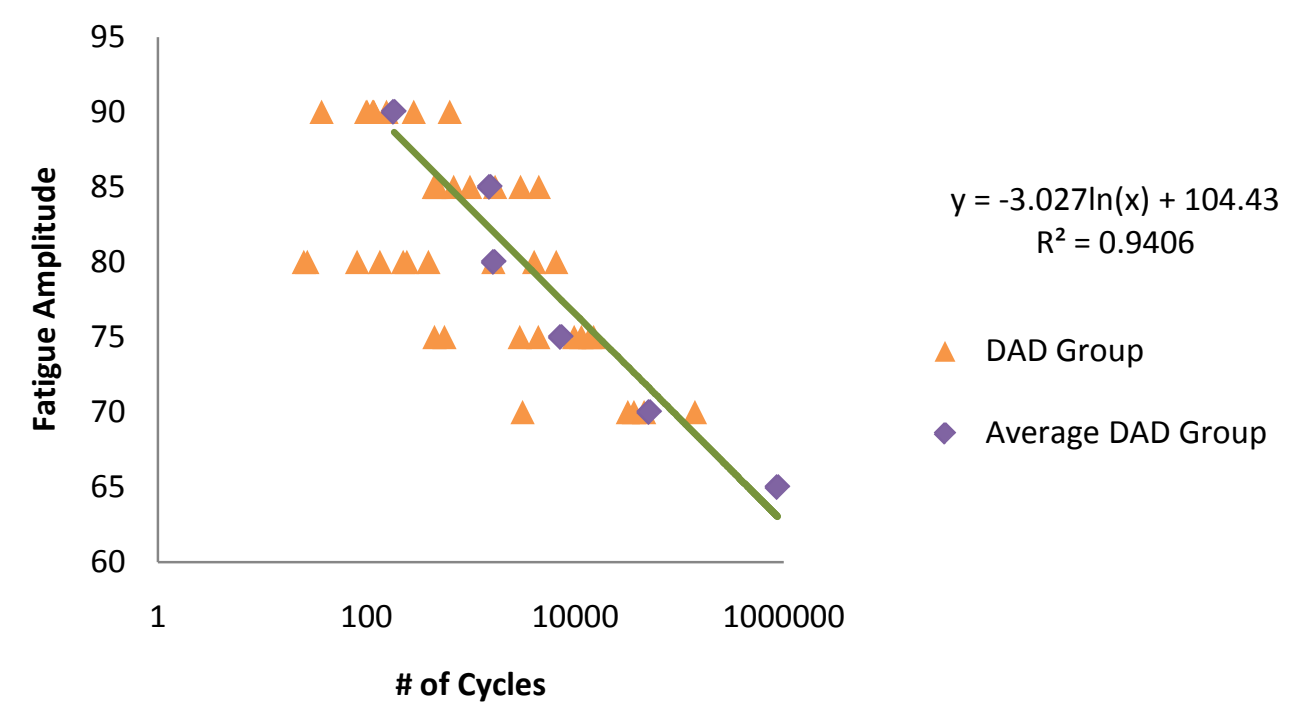

Figure 107: DAD Group Trend

The standard deviation for each test group ranged from $81 \%$ to $166 \%$. The huge standard deviation was due to the manufacturing defects of the test specimens. Similar to the previous section, the trend of the material failure of the specimen was more important than the accuracy of the results.

The results showed that specimens with many manufacturing defects tended to fail quicker. A lot of the test specimens were clumped to the left side of the chart, due to the defects of the specimens. Specimens that had an ideal failure result are on the right side of the test results. The results show that the results were in the extremes of the material.

The averages of the specimens were calculated to be used for the trend line of the material. The trend line for the control group fatigue testing had a coefficient of determination of 0.9406, showing that a logarithmic trend was ideal for the material property. The test results in a Cartesian scale show that the endurance limit of the material was between $65 \%$ to $70 \%$ fatigue amplitude of the yield stress; this can be seen in Figure 108.The trend shows that the number of 
failures levels out after amplitude of $70 \%$ was reached. This was helpful for designing a system with the use of the material.

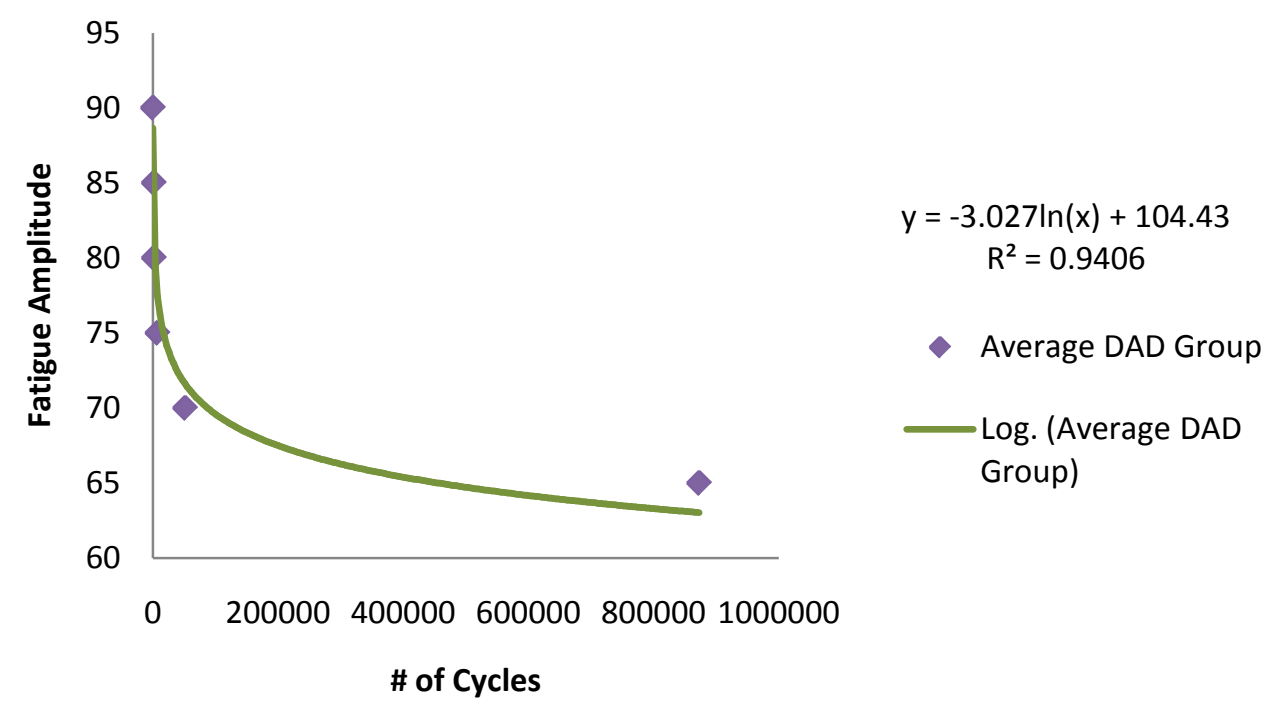

Figure 108: DAD Group Average Results

\subsection{Fatigue Trend}

The fatigue trend for the research was compared; this can be seen in Figure 109. The fatigue showed that the two trend lines were almost on top of one another, with its constituent results identical to one another. For each fatigue amplitude case, the number of failures was similar to both the control group and DAD group testing. This showed that the fatigue trend for both test groups was similar to one another. It shows that regardless of adding DADs to the specimen, the overall material properties were the same to each other. The addition of the DADs had no significant effect on the failure characteristics, but increased the strength of the material. 


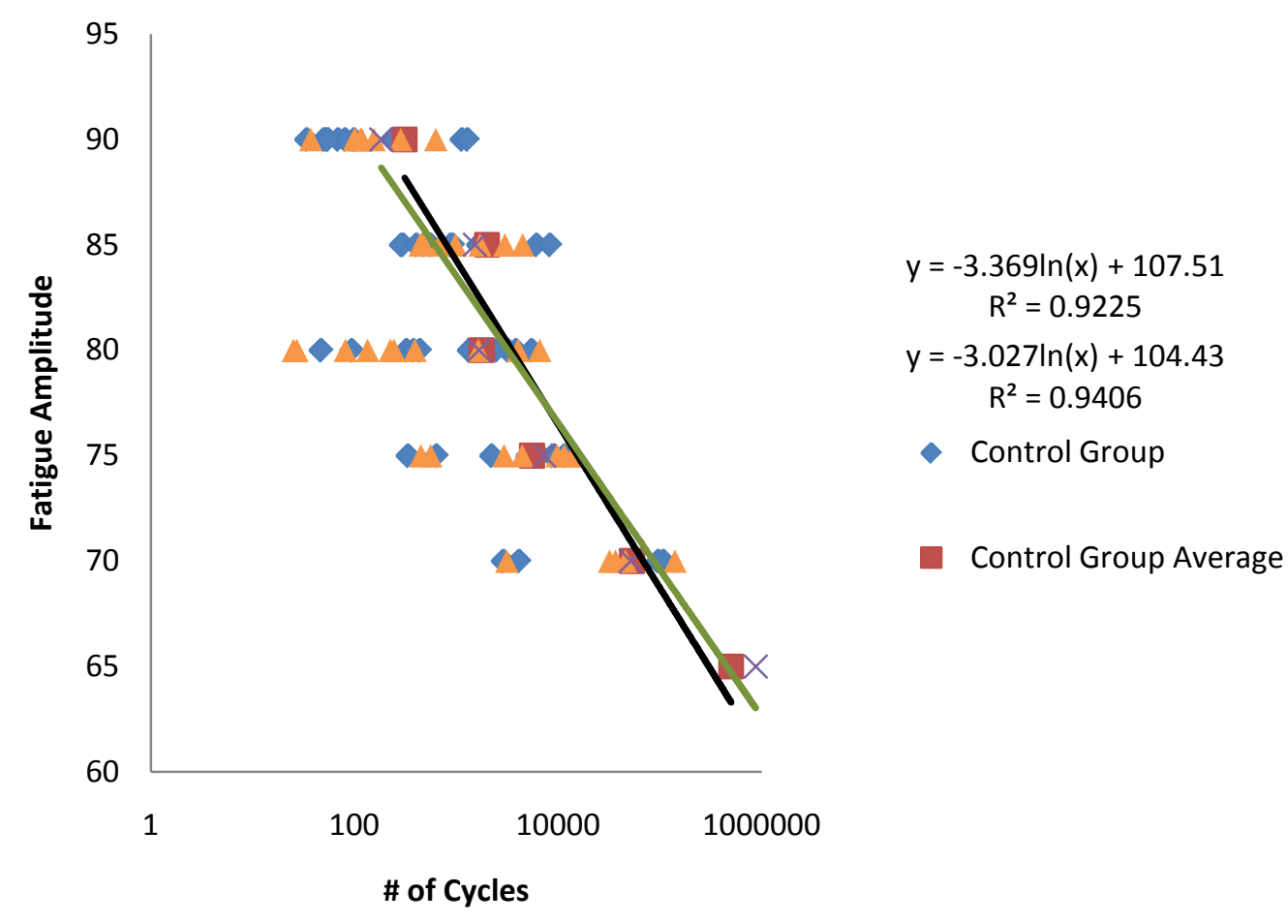

Figure 109: Fatigue Trend

The average test results showed that the specimens had similar endurance limit; this can be seen in Figure 110. It showed that the coefficient of determination for both groups was pretty good by both having values above 0.9. It showed that the drops for both specimens were identical but the part of the curve that asymptotes was different from one another. The DAD group showed a higher endurance limits, than the control group by its asymptote being a little higher. This could be due to the error in the testing for $65 \%$ fatigue amplitude. Only one test specimen was tested for each case because the length of test for each specimen was a day. If more specimens were tested,r the case could show that both groups asymptote at the same fatigue percentage. 


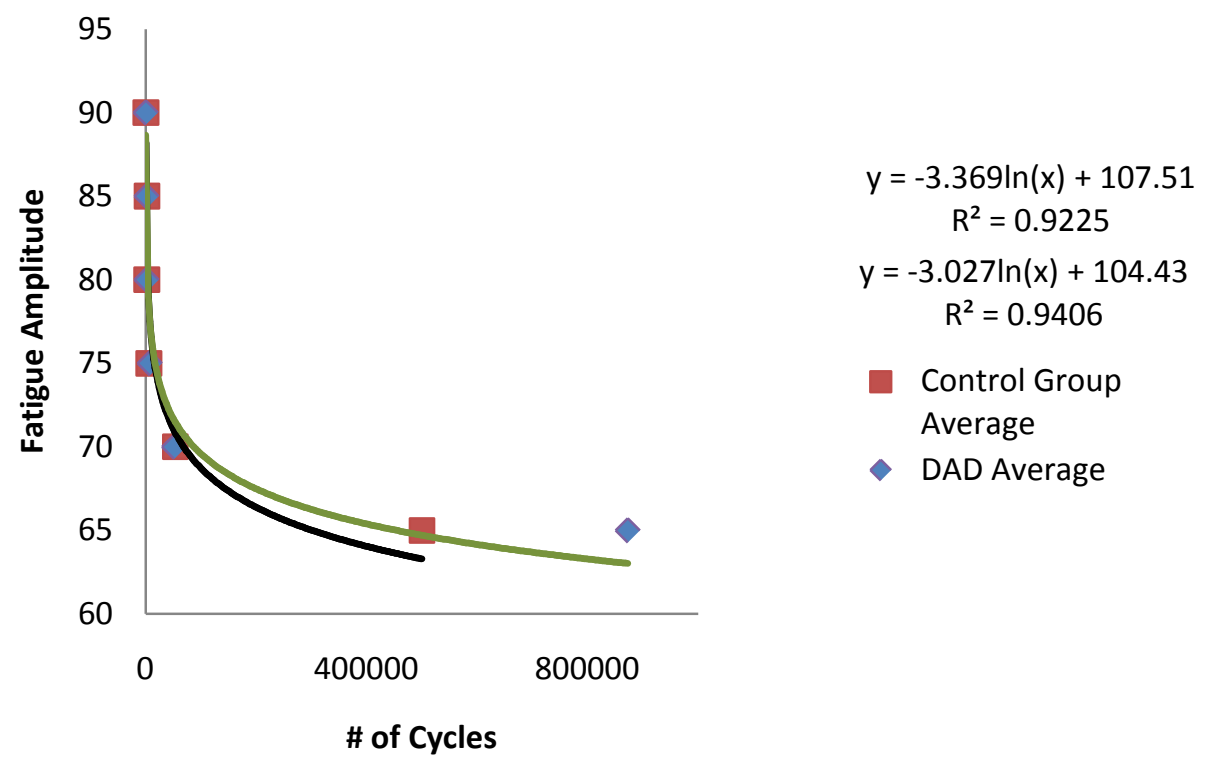

Figure 110: Average Fatigue Trend 


\section{Types of Failure of Composite Sandwich Panels}

\subsection{Static Loading Failures}

There are several types of failure seen in the static loading testing of the test specimens. For the control group and the DAD group, the most common failure seen was bearing stress around the fastener; this can be seen in Figure 111. The bearing stress on the specimen showed that the upper half of the hole had the specimen deforming around the fastener. The face sheet was crinkling around the fastener, by pushing some of the material out of the plane of the face sheet. This was the acceptable failure defined by ASTM D5961. The failure was localized and only appeared around the hole instead of causing a failure for the whole specimen. The overall structural integrity of the composite sandwich panel was still intact from the bearing stress.

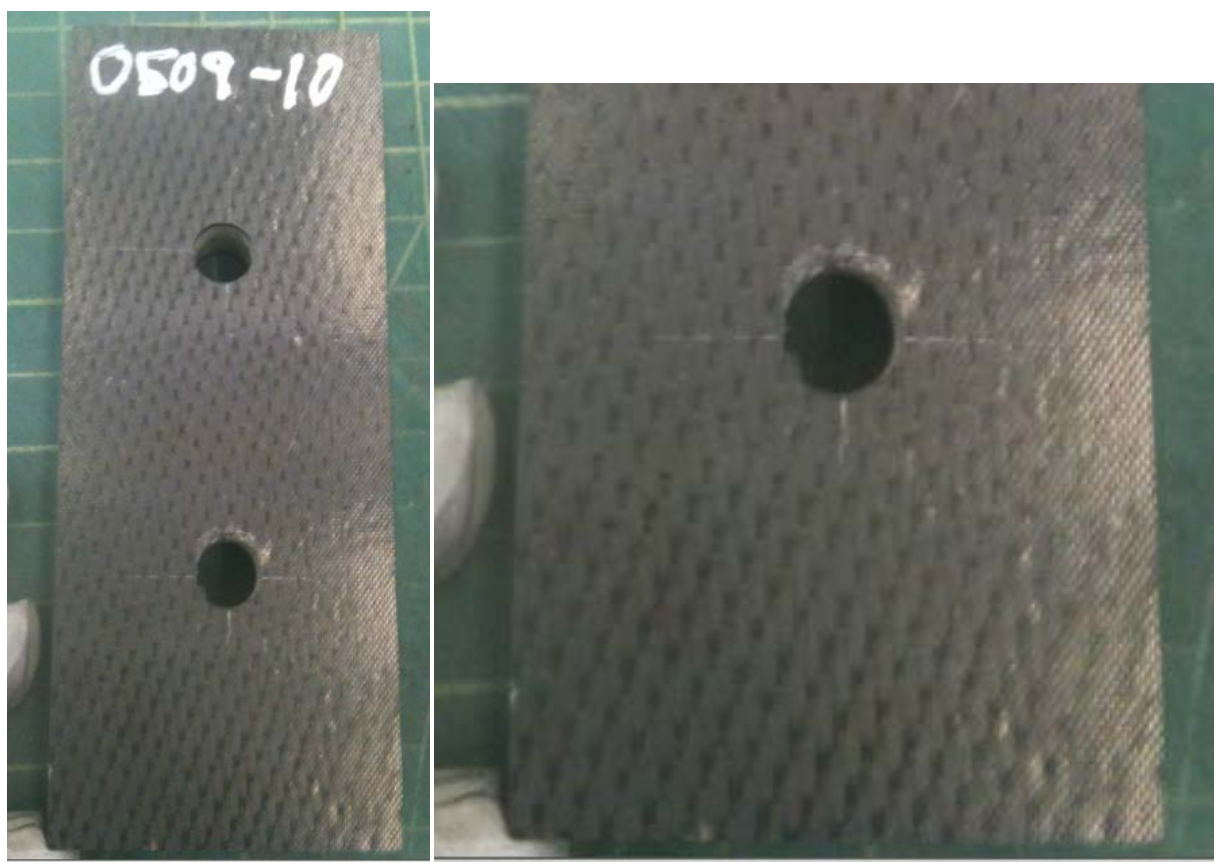

Figure 111: Bearing Stress Failure

A micro-buckling and bearing failure was seen on some of the test specimens; this can be seen in Figure 112. Bearing failure was still seen along the hole of the specimen, but was less pronounced than the previous failure. The failure had more of a micro-buckling of the individual fibers around the hole. The fibers were buckling one at a time, instead of the whole face sheet 
failing at once. This can be seen with a few fibers above the hole moving outward from the face sheet plane. The failure was a localized event but could propagate along the face sheet in the vertical direction of the specimen. This was an interesting and unexpected failure because the bearing stress affected individual fibers instead of the whole laminate.

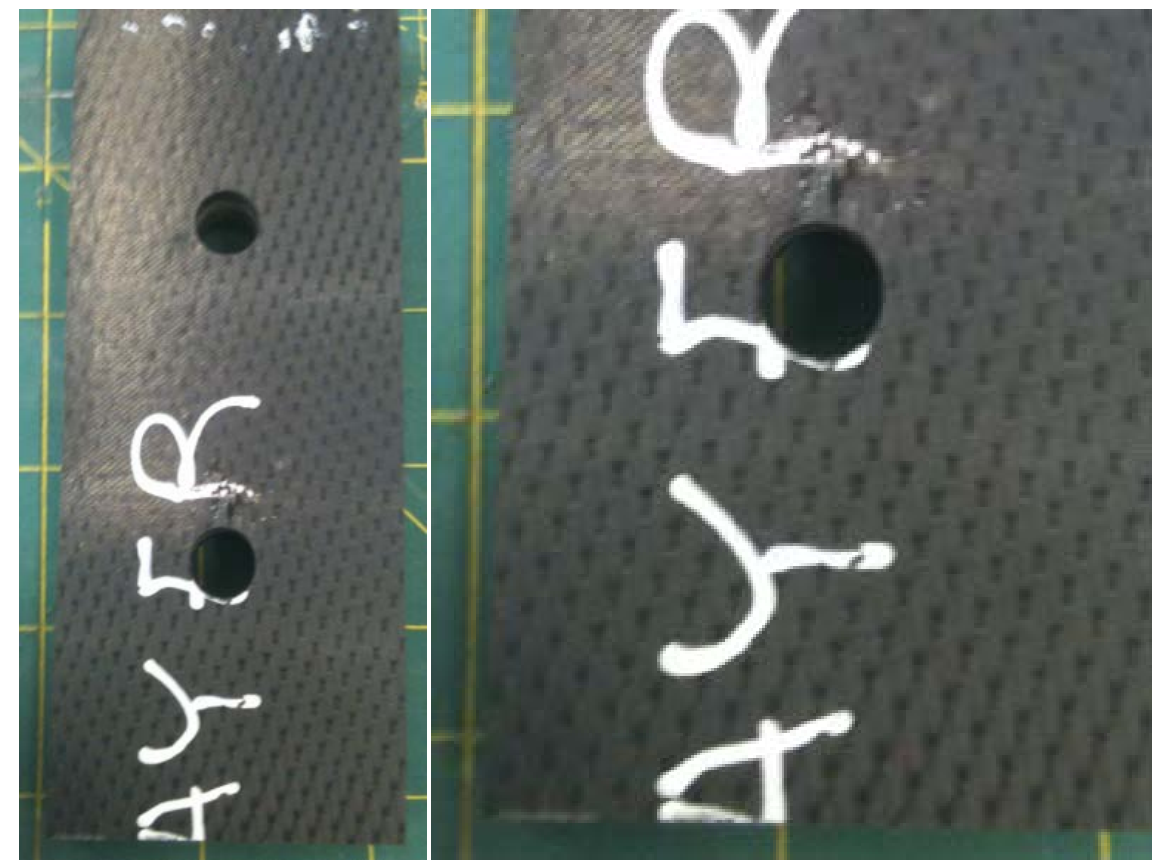

Figure 112: Bearing/Micro-buckling failure

Another type of bearing failure of the test specimens, mostly with higher thickness DADs can be seen in Figure 113. The failure was still a bearing stress around the hole, but the bearing failure was more evenly distributed along the DAD. The DAD was carrying the bearing stress from the fastener and was transferring it to the composite sandwich panel. It was delaying the bearing failure around the hole by transferring it into where the core of the panel meets the DAD. The specimen showed that the section of the DAD around the fastener was being pushed up causing fracture between the DAD and the face sheet. A small horizontal fracture can be seen above the hole, showing the DAD trying to "pop" out of the composite sandwich panel. The failure was still localized around the hole, but the failure can expand and take up the entire width of the composite sandwich specimen. 

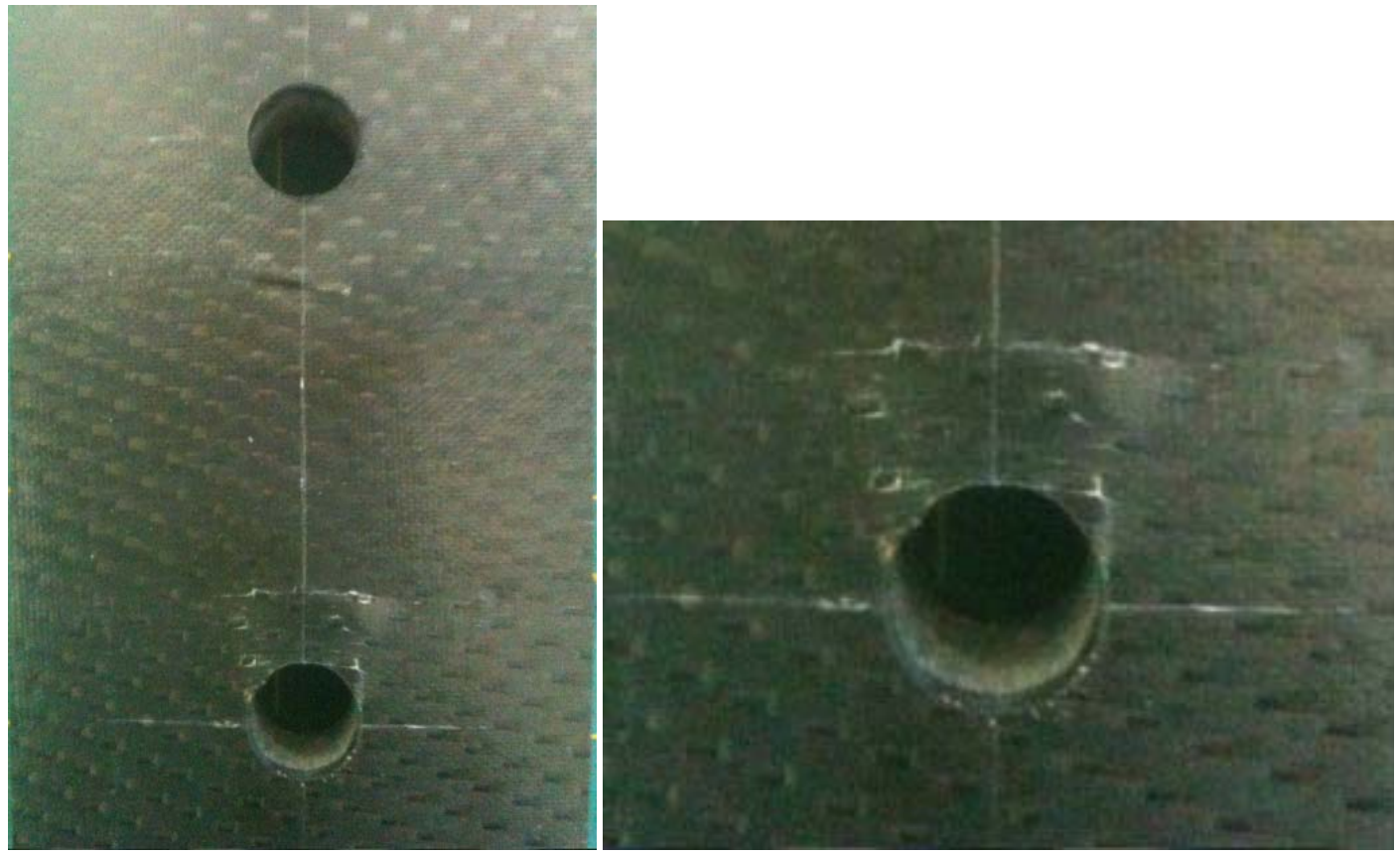

Figure 113: Bearing Stress on high thickness DADs

A complete failure of the specimen can be seen in Figure 114. The specimen was seen to have the face sheet being delaminated from both sides of the core at the edge of the DAD. The DAD was pushing on the core, causing the face sheet to delaminate. The fracture propagated between the face sheet and the core until it reached the other DAD on the specimen. The specimen had a full failure, rather than a localized failure, because the DAD was shearing along the core. The fracture can be seen with a horizontal line going along the width of the specimen at the bottom of the hole. The fracture follows along the fibers of the face sheet. The failure was usually a straight line along the width of the specimen. 


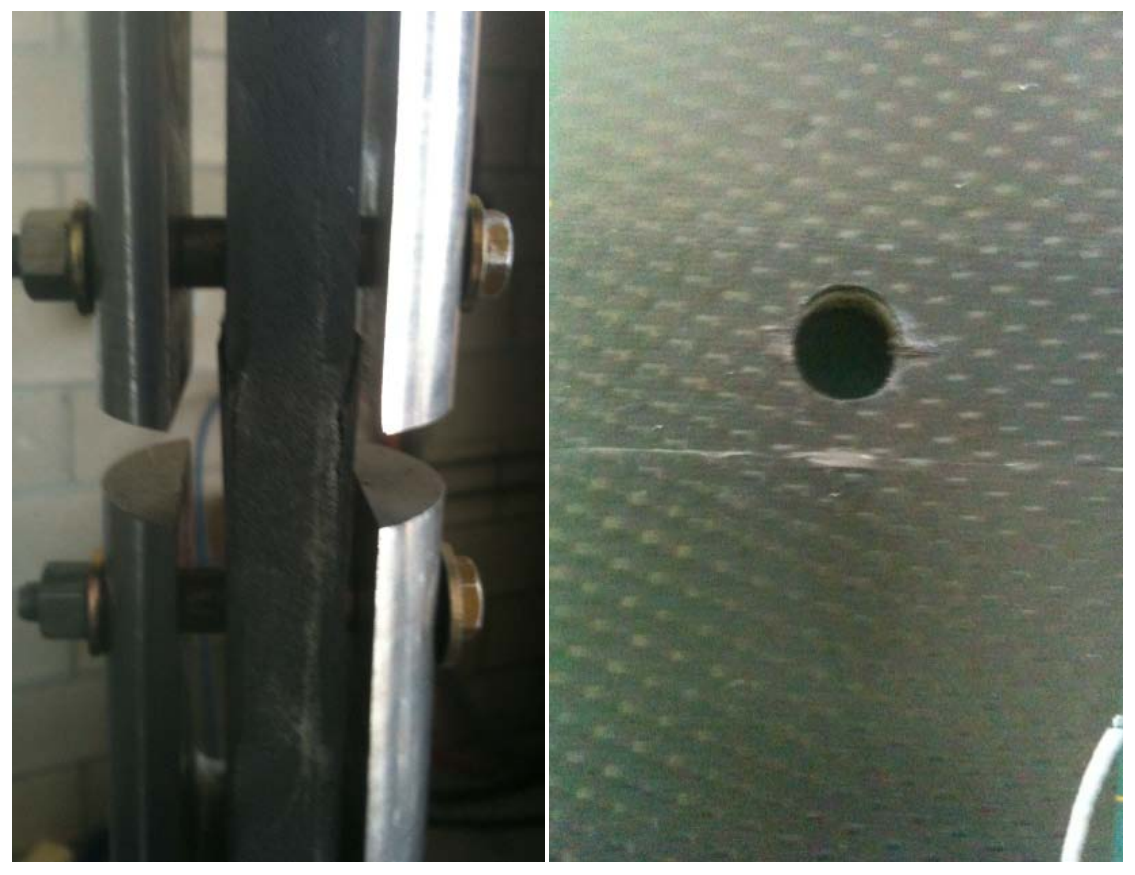

Figure 114: Complete failure of composite sandwich panel

A similar complete failure with buckling was seen for specimens with thicker DADs; this can be seen in Figure 115. The figure showed complete failure of the specimen with the face sheet above the DADs being completely delaminated from the core. The specimen was also buckling due to the failure seen on the specimen by giving way and buckling on the opposite side of the failure of the face sheet. The buckling could be due to the additional stiffness added around the fastener. The thicker DAD layers dominated the core by taking more than $25 \%$ of the thickness. 


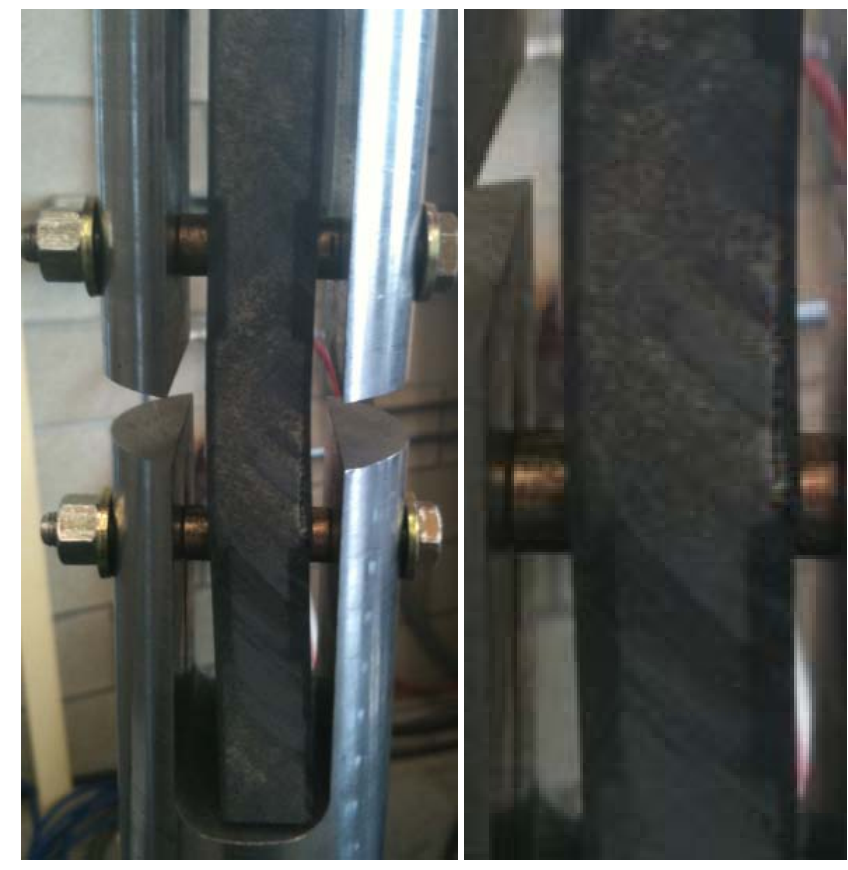

Figure 115: Complete failure with buckling of composite sandwich panel

The failures seen during monotonic testing showed that the specimen failure started out at a bearing failure to a complete delamination of the face sheet with buckling as the thicknesses of the DADs increased. This was due to the combined thickness of the DADs taking up a majority of the core thickness and was only being seen by the fastener through the composite sandwich panel.

\subsection{Fatigue Loading Failures}

There were several types of failure seen in the fatigue loading testing of the test specimens. For the control group and the DAD group, the most common failure seen was bearing stress around the fastener; this can be seen Figure 116. The bearing stress on the specimen shows that the upper half of the hole had the specimen deforming around the fastener. This was due to the machine stopping testing at set extension that was equivalent to the yield stress of the material. The face sheet was crinkling around the fastener, by pushing some of the material out of the plane of the face sheet. This was the acceptable failure defined by ASTM D5961. The failure was localized and only appeared around the hole instead of causing a failure for the whole 
specimen. The overall structural integrity of the composite sandwich panel was still intact from the bearing stress. Most of the failure during fatigue loading was this type of failure.

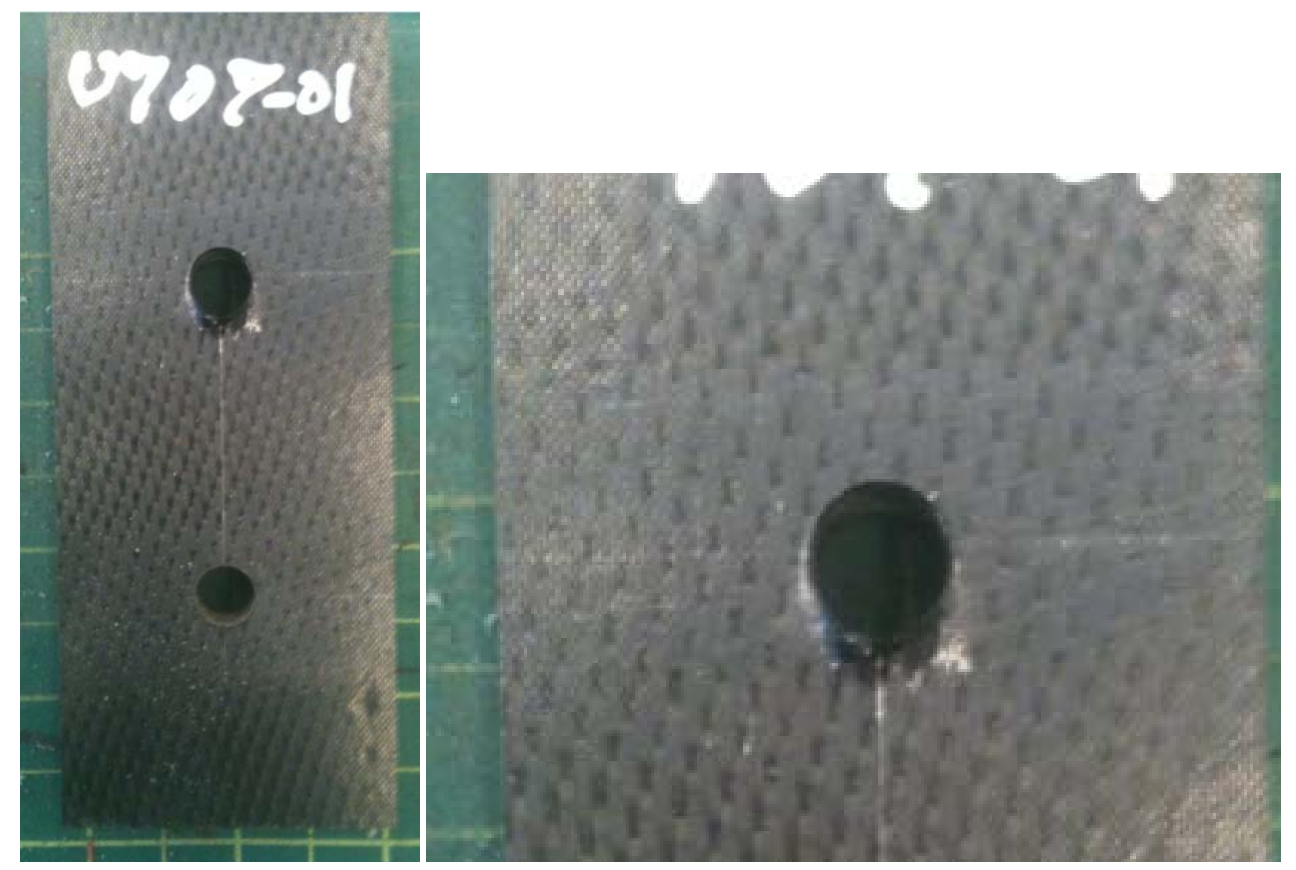

Figure 116: Bearing Failure at Fatigue

The other type of failure seen during fatigue testing was the fastener shearing along the composite sandwich panel; this can be seen in Figure 117. The fastener was applied a "sawing” motion along the composite sandwich panel by pulling through the composite sandwich panel with little resistance. This happened to some specimens when the testing was near the failure extension criterion and at high fatigue amplitudes. This happened because the specimens failed quicker by the load applied on the specimens being close to the yield load of the test specimens. This meant that it reaches the failure criterion quicker. This happened to both the control group and DAD group testing. 


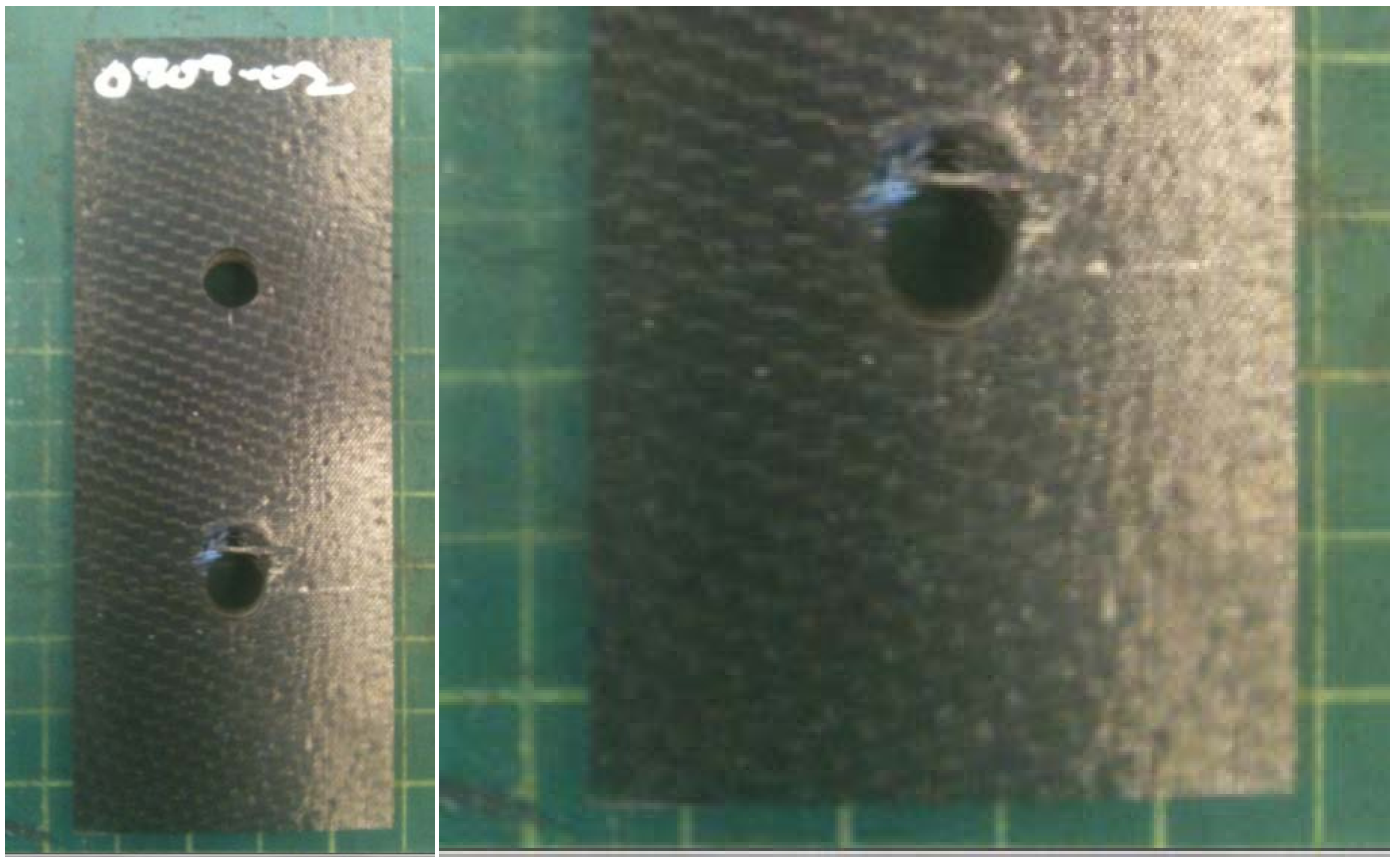

Figure 117: Shearing Failure during Fatigue Testing 


\section{Finite Element Modeling}

For this chapter, the FEM analysis of the composite sandwich panels will discussed. The creation of the model geometries and the mesh configurations for each model will be examined. The loading and boundary conditions applied to the models will also be talked about. Lastly, the displacement and stress distributions results of each model will be discussed.

\subsection{Finite Element Model Geometry}

The finite element model for the research contained three different models for each the control group and the DAD group for the analysis. The software that was used for the analysis was GeoStar or CosmosM, which was made by SolidWorks. The three different models consisted of just the test specimen, one with the test specimen and the bushing, and one with the test specimen, bushing and the fastener. The analysis had a base model to work from, which was just the test specimen because the other parts of the test were added to the model. The base model can be seen in Figure 118.
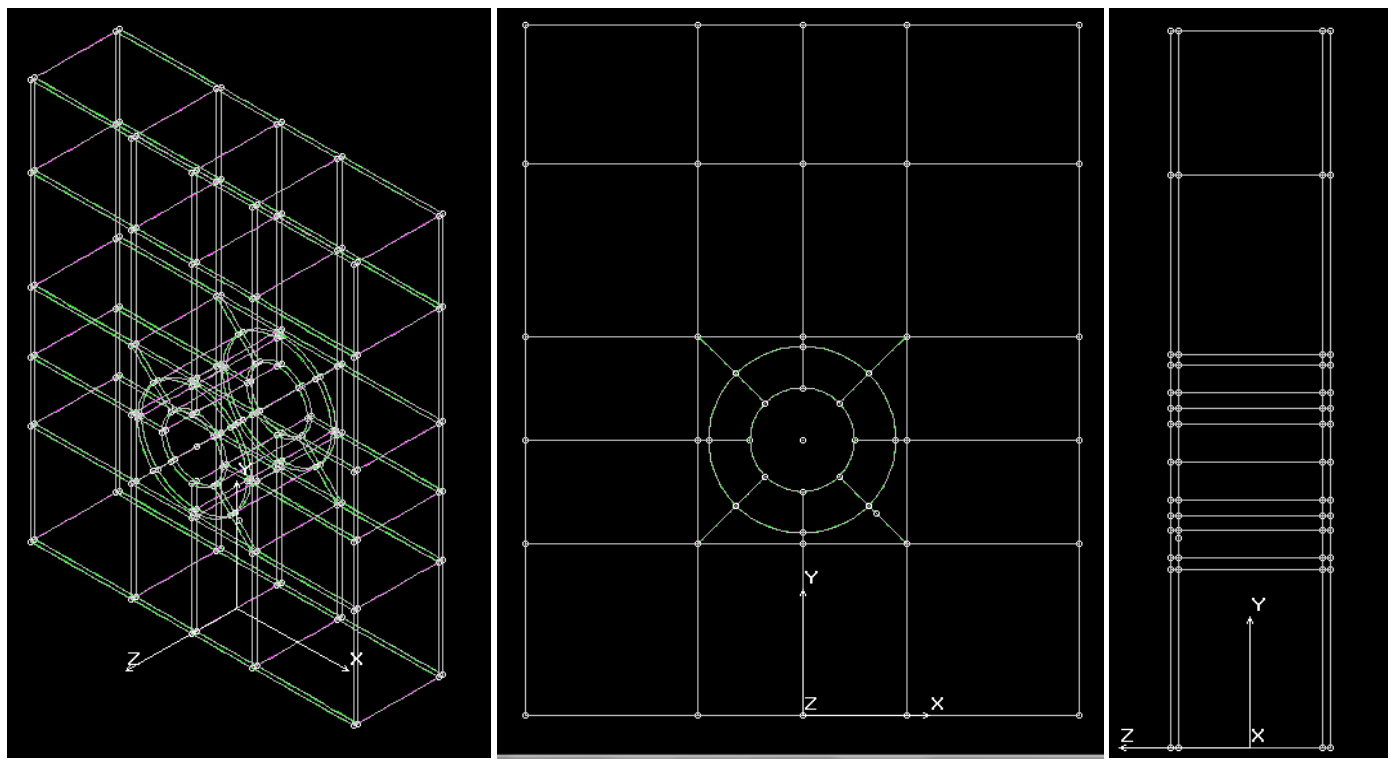

Figure 118: Base FEM Geometry

The FEM base model was the half model of the specimen, having only one hole rather than two, because of symmetry in the geometry. The geometry dimensions of the combine 
surfaces were $2.5 \times 2$. The software accepted values as unit less allowing the operator to nondimension the data. The units had to be recorded for further analysis. The base geometry contained 32 surfaces that were extruded into volumes. The surfaces around the hole were segments of circles and outside of them are triangle shaped quadrilaterals. All surfaces were quadrilaterals because the maximum surfaces that can be created in the software were with four lines/curves. The radius of the circular surface around the hole was $40 \%$ of the radius of the hole, which was $0.15 ”$. The purpose of this was to have a fine mesh of elements around the hole to capture the bearing failure and stresses from the fastener. Surfaces away from the hole were rectangles. The rectangular surfaces around the hole represent the DADs for later models. Doing this simplified the model and made it comparable to one another by having similar geometries and meshes. The 32 surfaces were then extruded into three volumes to represented the test specimen. The three volumes were the two face sheets and the core. The surfaces were extruded into the dimensions of each volume. The face sheet had a depth of 0.026 and the core had a depth of 0.5 . The model geometry would then be ready to mesh.

The next model that was created was the base model with the bushing inside the hole. The bushing was being added to the FEM model to alleviate the pressure on the test specimen; this can be seen in Figure 119. The half model of the bushing was only be modeled. The bushing had a radius of 0.0625 ” because the inner diameter of the bushing was 0.25 ” and the outer diameter of the bushing was 0.375 ”. 


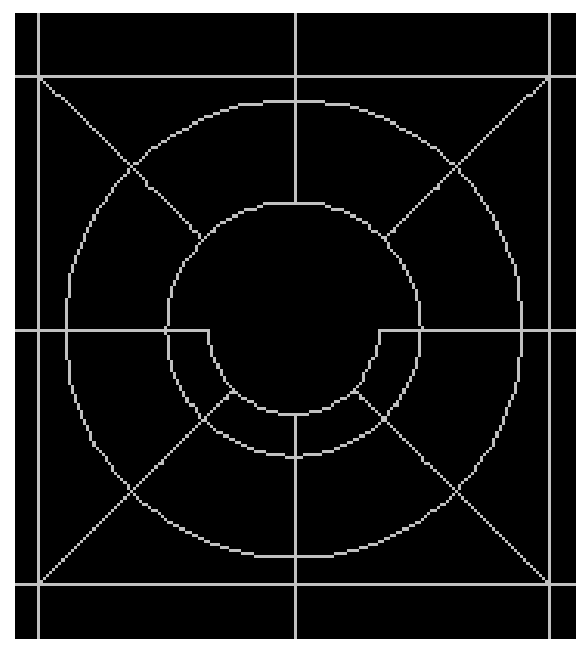

Figure 119: Base geometry with bushing

The last model for the control group FEM was the base model with the bushing and the fastener modeled; this can be seen in Figure 120. Just like the bushing, the fastener was added to the FEM model to simulate the true loading and geometry of the test specimens. The half model of the fastener was only modeled. The fastener had a radius of 0.125 because of the diameter of the fastener. The geometry was created by using the three surface features to make each surface of the model.

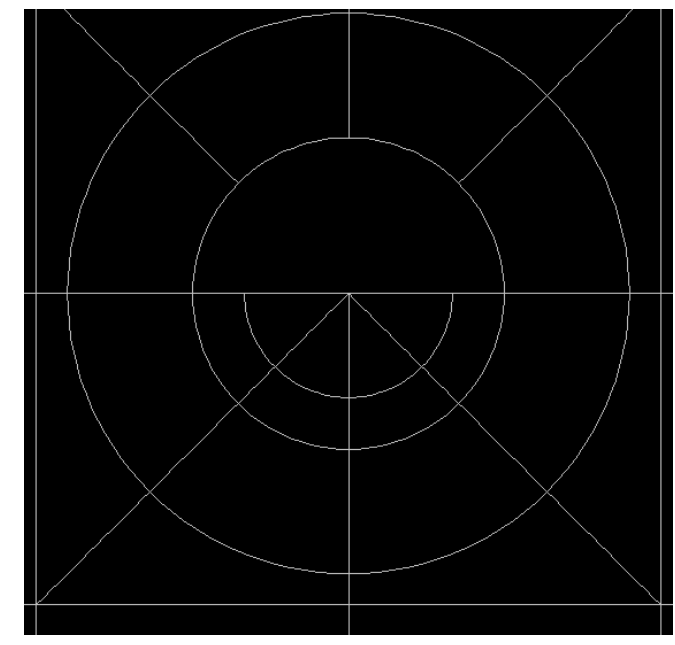

Figure 120: Base geometry with bushing and fastener

The model for the DAD geometry was different than the base geometry. The planar geometry was the same as the base model by having the same number of surfaces; this can be 
seen in Figure 121. The geometry had the same dimensions of the overall sandwich composite panel, a dimension of $2.5 \times 2$. The difference in the geometry was in the $\mathrm{Z}$ direction where the middle surfaces that represented the DAD were extruded into the core. The dimensions of the geometry were adjusted to accommodate the meshing of the elements. Since the thickness of each layer of LTM45 was 0.013”, the thickness of the material for the FEM model was a thickness of 0.02”. This made the thickness of the face sheet 0.04 ” and the thickness of the DAD 0.06”. This created an even number of elements in the core to match the number of elements with the DAD and the face sheet. The thickness of the core would remain the same. This geometry was the base model for the FEM of the DADs with the bushing and the fastener. The geometries for the DAD models with the bushing and the fastener were the same as the previous model. The bushing was added on the lower part of the hole on the composite sandwich panel and the fastener was added on top of the bushing. The half models of the bushing and the fastener was modeled.
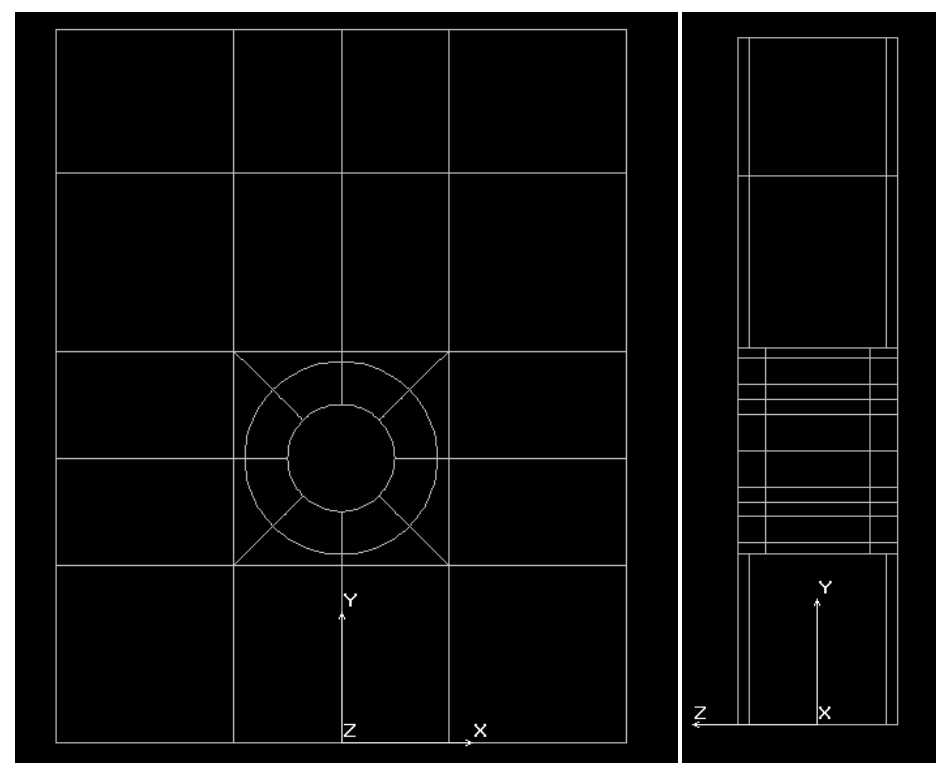

Figure 121: DAD geometry

\subsection{Finite Element Model Meshing}

The base model used a solid element to be able to calculate the strains and stresses inside the test geometry. The element mesh of the base model can be seen in Figure 122. All the 
elements for the model were quadrilateral elements. Two elements made up the face sheet in the orthogonal direction of the plane. Each element represented a layer of LTM45. Six elements made up the core in the orthogonal direction of the plane. Six were chosen because having too much made the element count too high and too long to calculate and too low would decreased the accuracy of the analysis. The mesh grew from a coarse mesh from the top edge to a fine mesh near the hole. A parametric mesh for each volume was done. This means that the user defined the number of elements in the $\mathrm{X}, \mathrm{Y}$, and $\mathrm{Z}$ directions of the volume. The figure was divided into groups to make it easier to describe the mesh densities of each volume/surface. Group 1 was the top horizontal surfaces. Group 2 was the next horizontal surfaces below Group 1. Group 3 represented the rectangular surfaces on both sides of the hole. Group 4 represented the circular sections and the triangle shaped rectangular surfaces. Group 5 represented the bottom horizontal surfaces of the geometry. The material properties of the LTM45 and the FR-6710, the experimental results, were used for the input for the elements for the face sheets and the core. The parametric meshing of each grouping can be seen in Table 30. The total element count for the whole geometry was 25,000 elements with 40,197 nodes. A majority of the elements were contained in Group 5 due to the fine mesh needed to capture the bearing failure around the hole. 


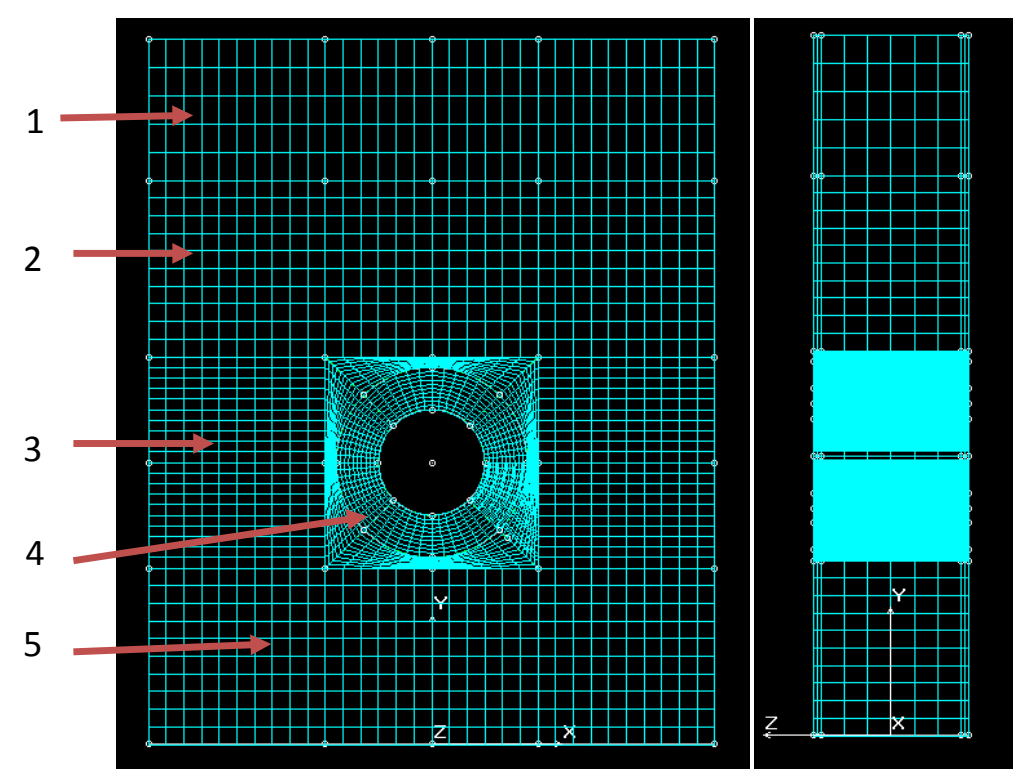

Figure 122: Base model element mesh

Table 30: Base Model Parametric Mesh

\begin{tabular}{|l|c|c|c|}
\cline { 2 - 3 } \multicolumn{1}{c|}{} & \multicolumn{3}{c|}{ Parametric Volume Meshing } \\
\cline { 2 - 3 } \multicolumn{1}{c|}{} & $\mathrm{X}$ & $\mathrm{Y}$ & $\mathrm{Z}$ \\
\hline Group 1 & 32 & 5 & \multicolumn{1}{|c|}{$\mathrm{Z}$} \\
\hline Group 2 & 32 & 10 & 2 for Face sheet and 6 \\
for Core
\end{tabular}

The base model with a bushing was meshed; which can be seen in Figure 123. Everything but the bushing geometry had the same element mesh as before. The bushing material property that was used for the mesh was the bronze material property that was standard in the software. The two outside volumes, or the top volumes, would have a parametric mesh of ten elements in the $\mathrm{X}$ direction and a parametric mesh of ten elements in the $\mathrm{Y}$ direction. The two inside volumes, or the bottom volumes, would had a parametric mesh of six elements in the $\mathrm{X}$ direction and ten elements in the $\mathrm{Y}$ direction. In the $\mathrm{Z}$ direction of the mesh, there were a total of 10 elements for the whole geometry. The mesh had a total of 28,800 elements, the bushing only adding 3,800 elements to the base model. 


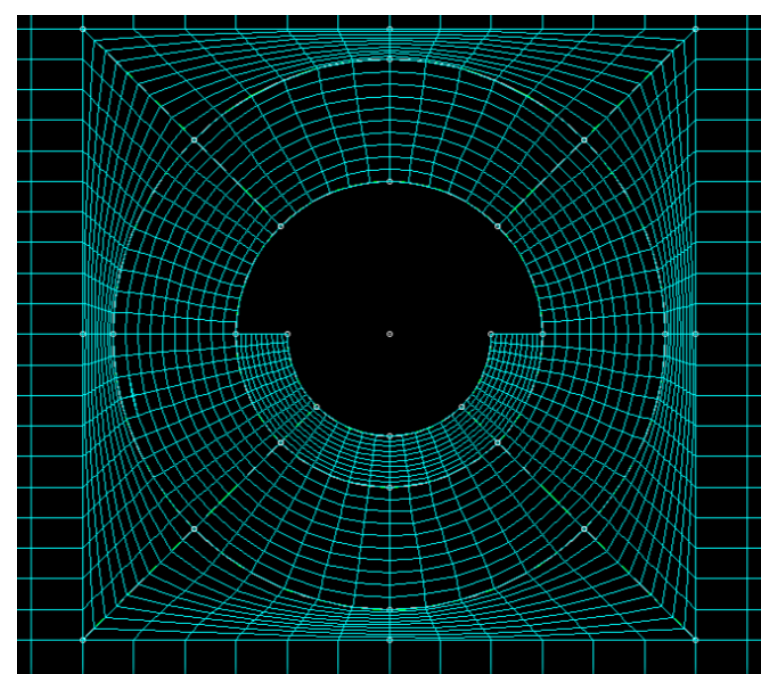

Figure 123: Base model with bushing element mesh

The base model with a bushing and a fastener was meshed; which can be seen Figure 124. Everything but the fastener geometry had the same element mesh as before. The fastener material property that was used for the mesh was the steel material property that was standard in the software. The two outside volumes, or the top volumes, had a parametric mesh of ten elements in the $\mathrm{X}$ direction and a parametric mesh of six elements in the $\mathrm{Y}$ direction. The two inside volumes, or the bottom volumes, had a parametric mesh of six elements in the $\mathrm{X}$ direction and ten elements in the $\mathrm{Y}$ direction. In the $\mathrm{Z}$ direction of the mesh, there were a total of 10 elements for the whole geometry. The mesh had a total of 30,000 elements, the bushing only adding 3,800 elements to the base model. The fastener added 1,200 elements to the model with the bushing and added 5,000 elements to the base model. 


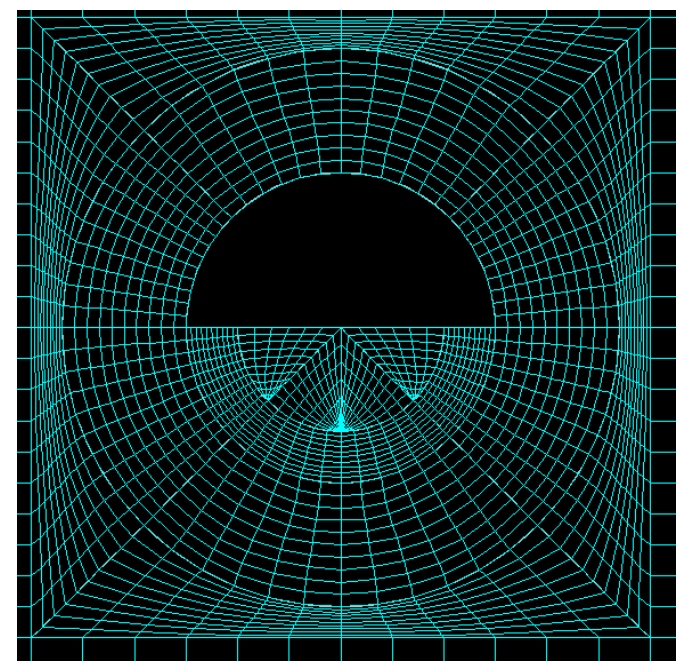

Figure 124: Base model with bushing and fastener element mesh

The DAD models had the same mesh for the sandwich plane, the $\mathrm{X}$ and $\mathrm{Y}$ direction that was talked about for the base model. The only difference in the meshing between the DAD models and the base models was the number of elements in the $\mathrm{Z}$ direction; this can be seen in Figure 125. The number of elements for the face sheet in the $\mathrm{Z}$ direction remained the same having two elements. The DADs sections had 3 elements in the $\mathrm{Z}$ direction. For the sections of the core that was only between the face sheets without the DADS, the number of elements in the $\mathrm{Z}$ direction was 25. For the sections of the core that were between the DADs there were19 elements in the $\mathrm{Z}$ direction. The sections of the core between the DADs have only 19 elements because the remaining six elements came from the DADs. Having four times the amount of elements in the $\mathrm{Z}$ direction greatly increased the total element count of the model. The DAD model had a total of 73,420 elements for the whole model. This was a huge increase from the base model, which had a total of 25,000 elements. An increase of 48,420 elements was mostly in the core of the model. This increase of elements would not affect the results. It actually made the analysis more accurate, but it took a lot longer to run the analysis than the base model. The main key for this analysis was that the same meshing method was used for the DAD models with the bushing and fastener. 


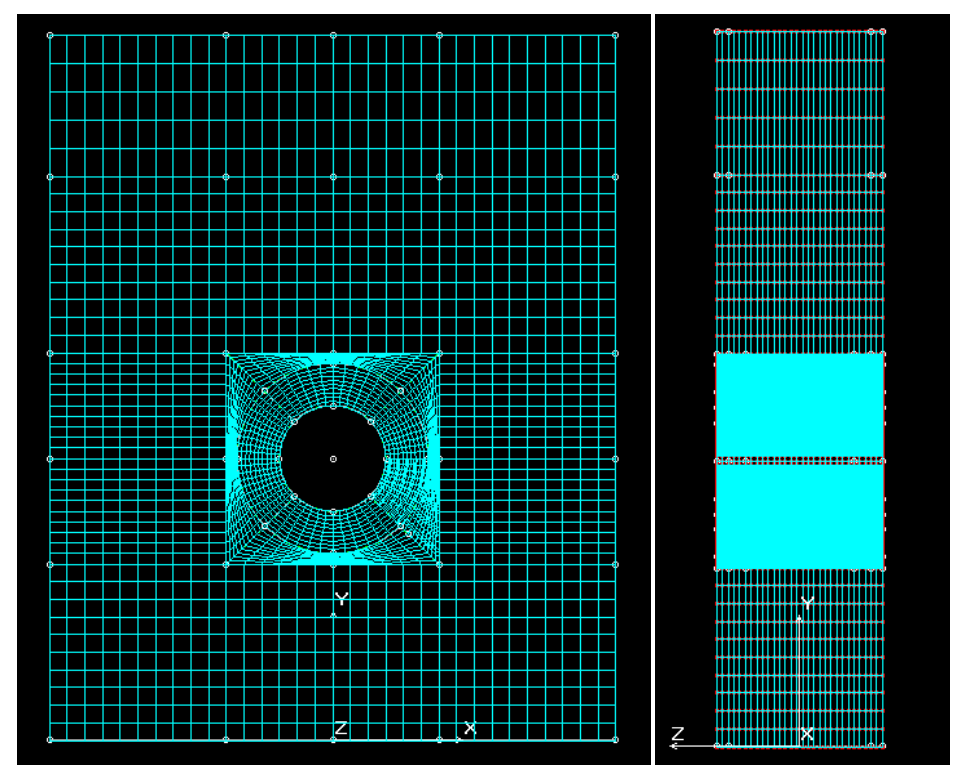

Figure 125: DAD model element mesh

The element meshing scheme for the DAD models with the bushing and fastener remained the same as before. The $\mathrm{X}$ and $\mathrm{Y}$ direction meshing scheme were the same, but the $\mathrm{Z}$ direction differed by having more elements like the base DAD model. The DAD model with a bushing had a total of 81,200 elements. This was an increase of 7,780 elements with the addition of the bushing. The DAD model with the fastener had a total of 88,160 elements. This was an increase of 6,960 elements.

\subsection{Finite Element Model Static Loading/Displacement}

The loading forces and the displacement constraints were added to the geometry. The displacement constraint was added on the bottom surface of the geometry to represent the symmetry of the specimen; this can be seen in Figure 126. Since the FEM geometry was a half model of the test specimen, it can be assumed that on the symmetry plane of the test specimen, the specimen would not move in the $\mathrm{X}, \mathrm{Y}, \mathrm{Z}$ directions and would have no moments acting on it. Displacement constraints were necessary so deformation displacements could be created from the loads applied on the test specimen. Without displacement constraints, the specimen just moved as 
one piece and not deform. The displacement constraints were applied to all FEM models, including the models with and without DADs, at the bottom surface.
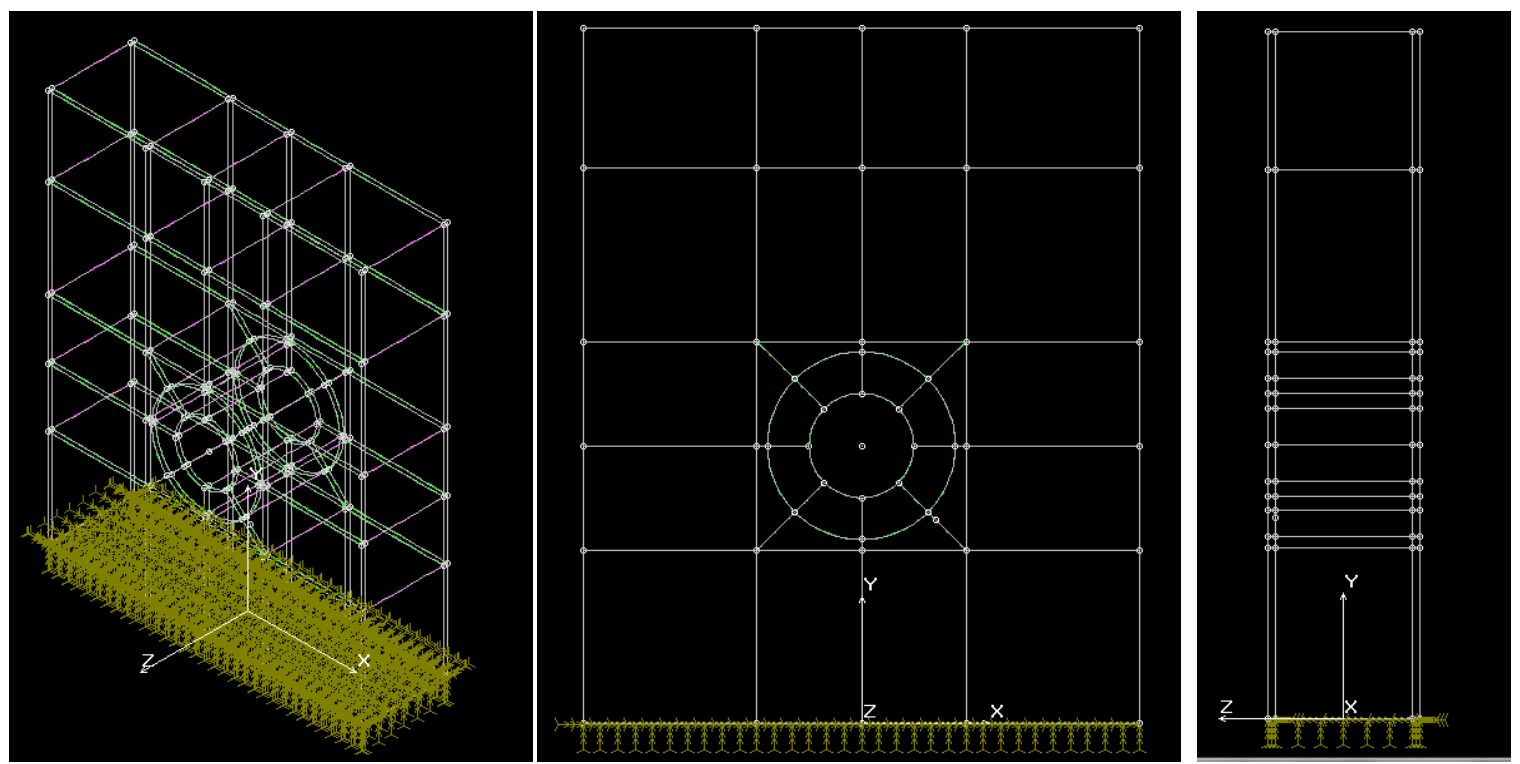

Figure 126: Base Model Displacements

The loading on the specimens was then added to the model; this can be seen in Figure 127. The loading was applied on the bottom surfaces of the cut-out hole section of the test specimens. The loading applied was a pressure force, with a pressure of -2,891 psi, which applied a monotonic load of -940 lbs. The loading for specimens with a DAD had a pressure force of 5081 psi, which applied a monotonic load of $-1,652 \mathrm{lbs}$, applied to the same surfaces. This applied load was equivalent to the yield load found during experimental testing. The pressure would only be applied in the vertical or Y direction to try to capture the real life loading seen on the test specimens. The overall displacement/extension of the FEM was then compared to experimental results to see the accuracy of the model. 

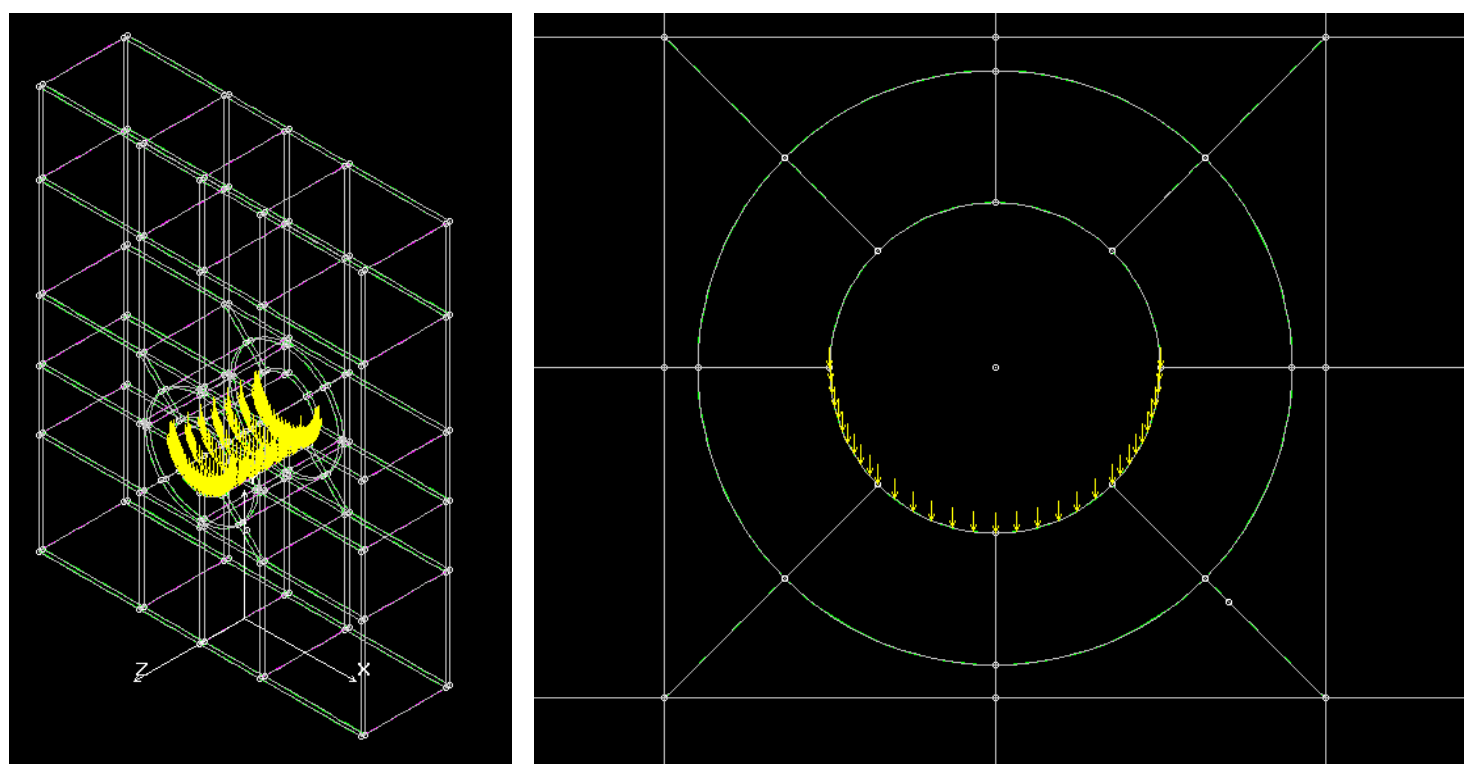

Figure 127: Base Model Loading Forces

The loading applied for the base model with the bushing was applied directly on the inner diameter of the bushing; this can be seen in Figure 128. The loading applied was a pressure force, with a pressure of $-2,891 \mathrm{psi}$, which applied a monotonic load of -940 lbs. The loading for specimens with a DAD had a pressure force of -5081 psi, which applied a monotonic load of 1,652 lbs, applied to the same surfaces. This applied load was equivalent to the yield load found during experimental testing. The pressure was only applied in the vertical or Y direction to try to capture the real life loading seen on the test specimens. 


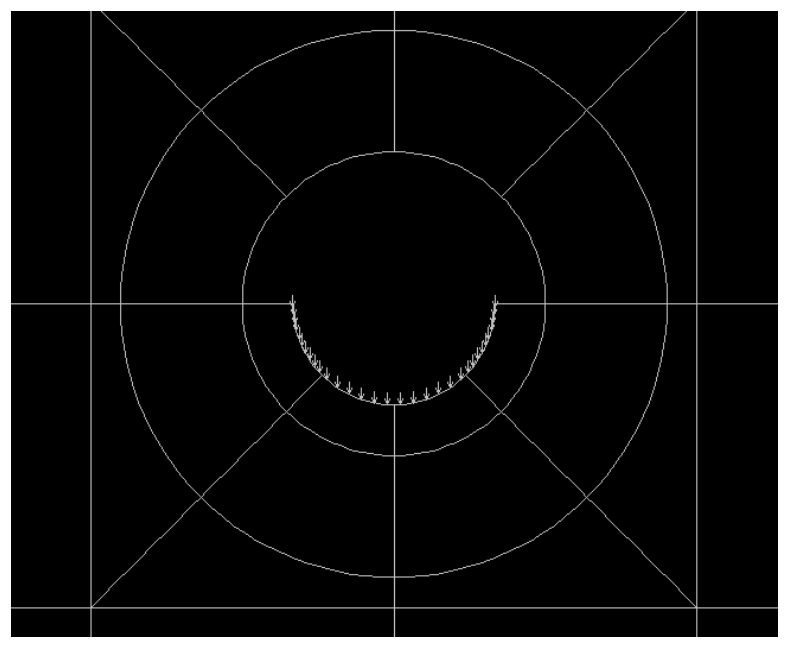

Figure 128: Base model with bushing loading forces

The loading applied for the base model with the bushing was applied directly on the inner diameter of the bushing; this can be seen in Figure 129. The loading applied was a pressure force, with a pressure of $-2,891 \mathrm{psi}$, which applied a monotonic load of -940 lbs. The loading for specimens with a DAD had a pressure force of -5081 psi, which applied a monotonic load of 1,652 lbs, applied to the same surfaces. This applied load was equivalent to the yield load found during experimental testing. The pressure was only applied in the vertical or Y direction to try to capture the real life loading seen on the test specimens.

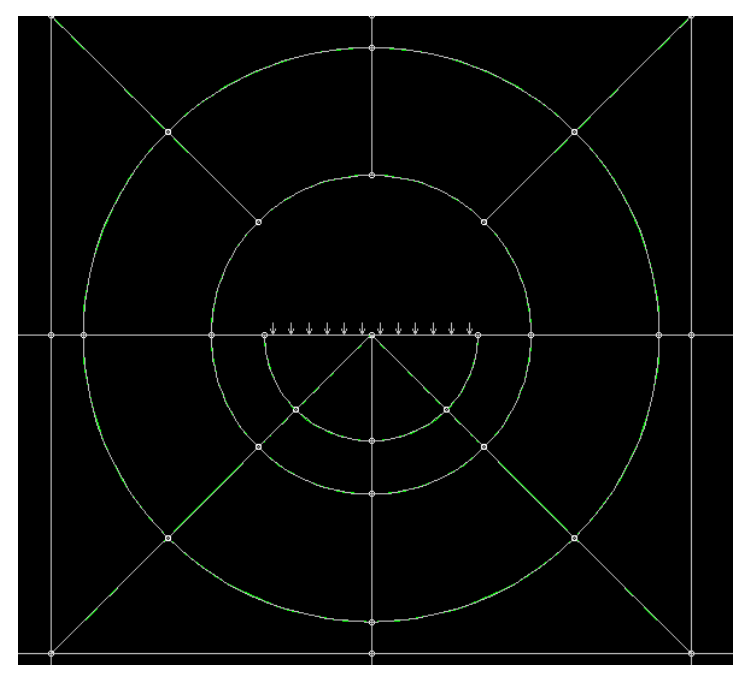

Figure 129: Base model with bushing and fastener loading forces 
From the results obtained for the pressure loading, the results for the bushing and fastener models gave very small displacement results from the experimental testing. The results obtained were all a power of ten smaller than the experimental value; this will be talked further in 8.4 Finite Element Model Static Results. The decision that was made was to change the loading on each of the models from a pressure loading to a force loading. Instead of applying the loads along the bottom surface of the hole, the loads were applied to the center line of the hole; this can be seen in Figure 130. The force applied was the force obtained from experimental testing, which was -940 lbs for non-DAD models and -1652 lbs for DAD models. This loading was applied to the remaining models to see if there was any significant improvement in the results of the models.
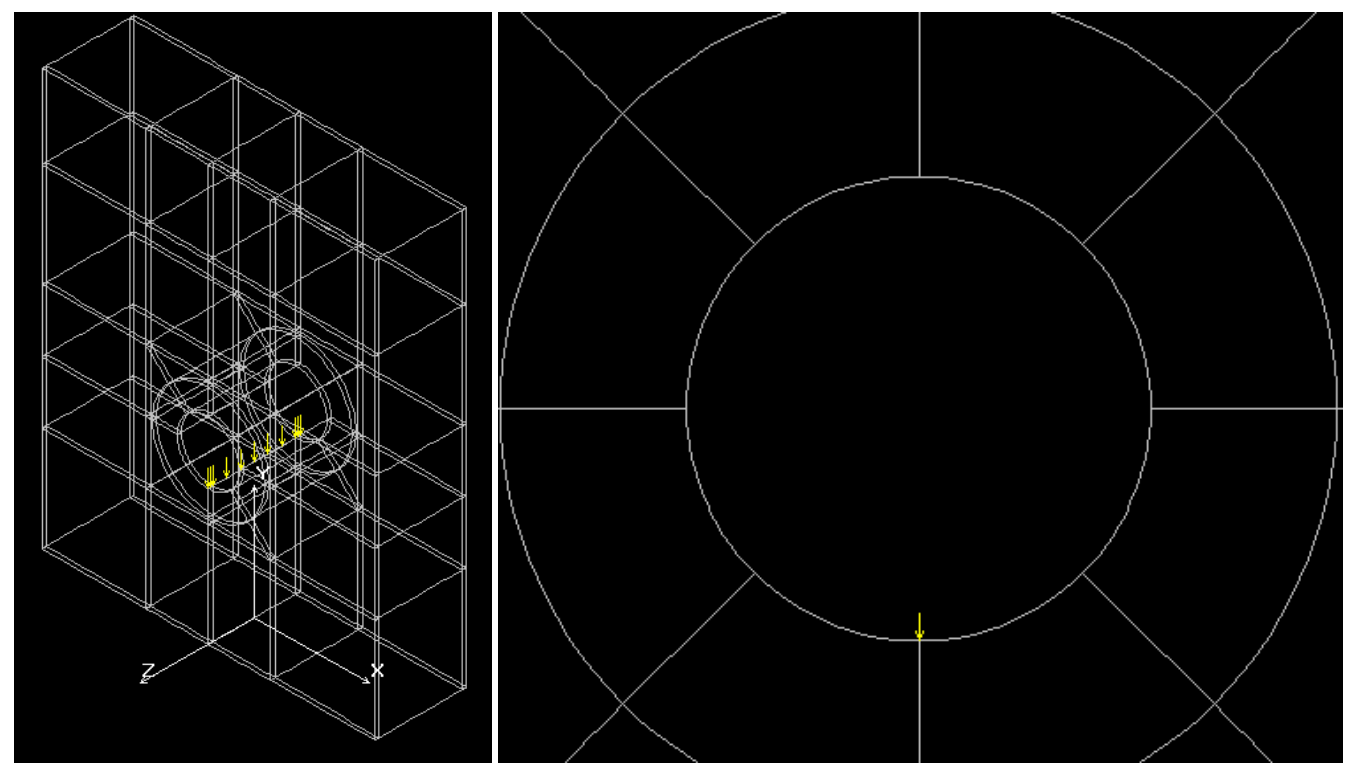

Figure 130: Force Loading on FEM Base Model

\subsection{Finite Element Model Static Results}

The results of the models for all static results were then analyzed. The first analysis was the base FEM model for the control group. The displacement response of the model can be seen in Figure 131. The stress response of the model can be seen in Figure 132. The model showed that nothing was happening with the face sheets in both the displacement and stress figures. Most of the movement in the model was occurring in the foam. The foam was being displaced from the 
constant pressure and was trying to push on the face sheet. This assumption can be backed up with the upward deformation of the foam seen in the displacement figure. The foam should be moving downward but instead was sliding up the face sheet. The foam cannot penetrate through the face sheet because it was a much stronger material than the core.

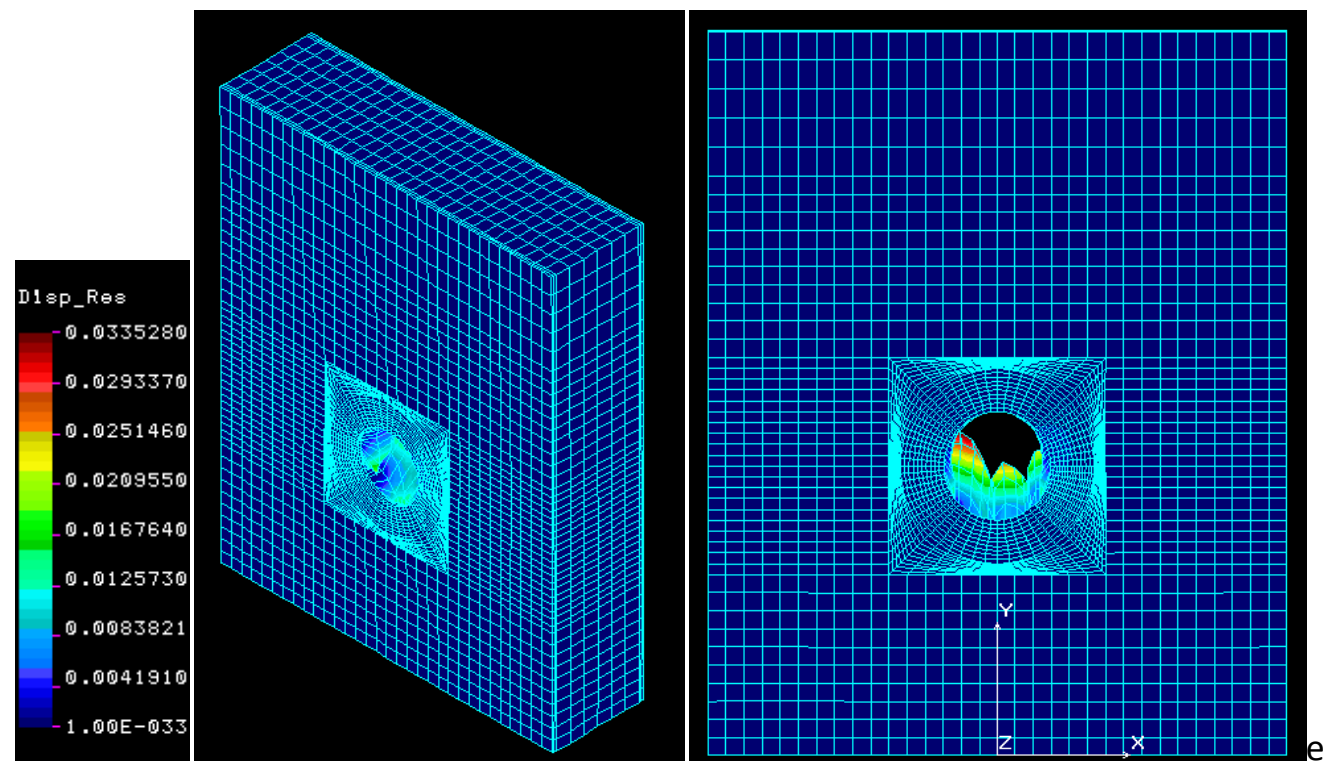

Figure 131: Displacement response of FEM base model with pressure loading

The stress distribution of the model showed that a circular stress concentration was seen at the bottom of the hole and was seen around the hole. The stress distribution at the bottom of the hole could be from the stress from the core trying to push out into the face sheet. An interesting stress concentration can be seen on the top corners of the meshing configuration on top of the hole. This could be due to how the mesh was created around the hole. The elements might not be transferring the loads properly at that area. Another reason the stress concentration was appearing was that there was a counter reaction of the face sheets and the core from the pressure loading. 


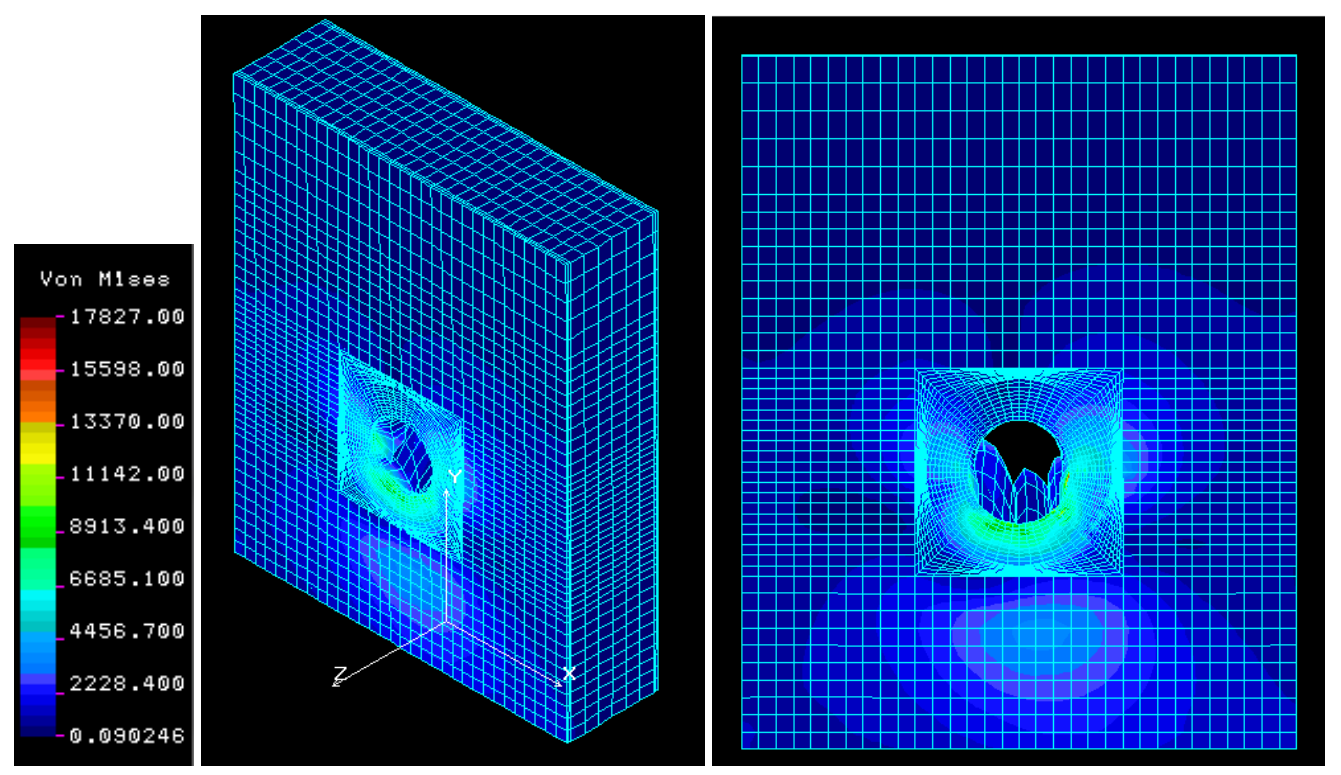

Figure 132: Stress response of FEM base model with pressure loading

The next analysis was the base model with the bushing for the control group. The displacement response of the model can be seen in Figure 133. The stress response of the model can be seen in Figure 134. The displacement response of the model resembled more what happened during experimental testing. The section below the hole was actually displaced in the planar direction of the model, showing a bulge on the model. The bushing prevented the core from moving upward, like the previous model. Instead the core was forced to move downward and push out into the face sheet to create the bulge on the model. A semi-circular displacement distribution was also seen at the bottom of the hole from the loading seen from the bushing. The addition of the bushing severely affected the displacement of the model, even though the failure mode was similar to experimental results. The displacement contour bar shows that the maximum displacement seen in the model was 0.00278 in, which was a complete order of magnitude below the actual results. It seems that the bushing was dissipating too much of the loading seen on the model. The actual sandwich composite sandwich panel could have seen a lot less force than what was applied. 


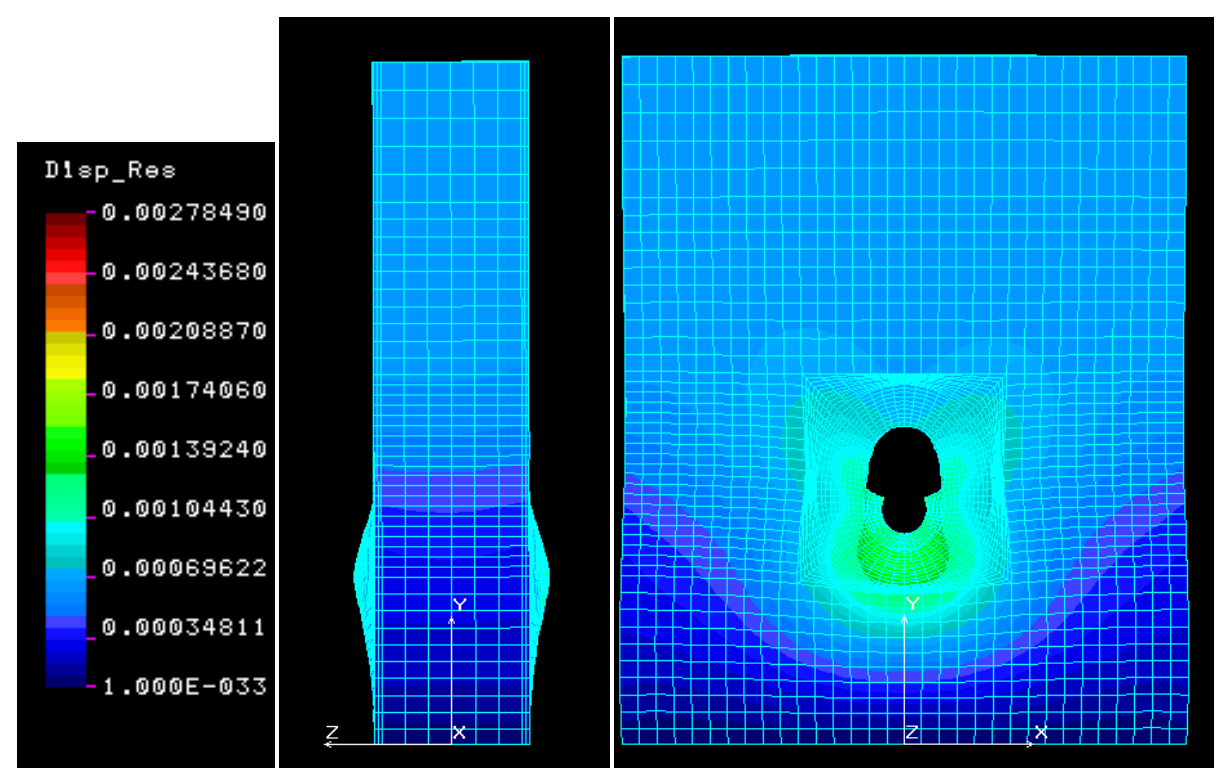

Figure 133: Displacement response of FEM base model with bushing and pressure loading

The stress distribution of the model with the bushing was similar to the base model. A more pronounced stress concentration was seen at the bottom of the hole than before. Instead of a circular shape, the stress concentration at the bottom of the hole stretched from the bushing all the way to the bottom of the model. This backs up the assumption made earlier that the core was pushing along the face sheet at the bottom of the hole. With the addition of the bushing, the stress concentration was actually able to push out onto the face sheet. The stress concentrations above the hole had disappeared, but new stress concentrations were seen at the tip of the bushing and the composite sandwich panel. 


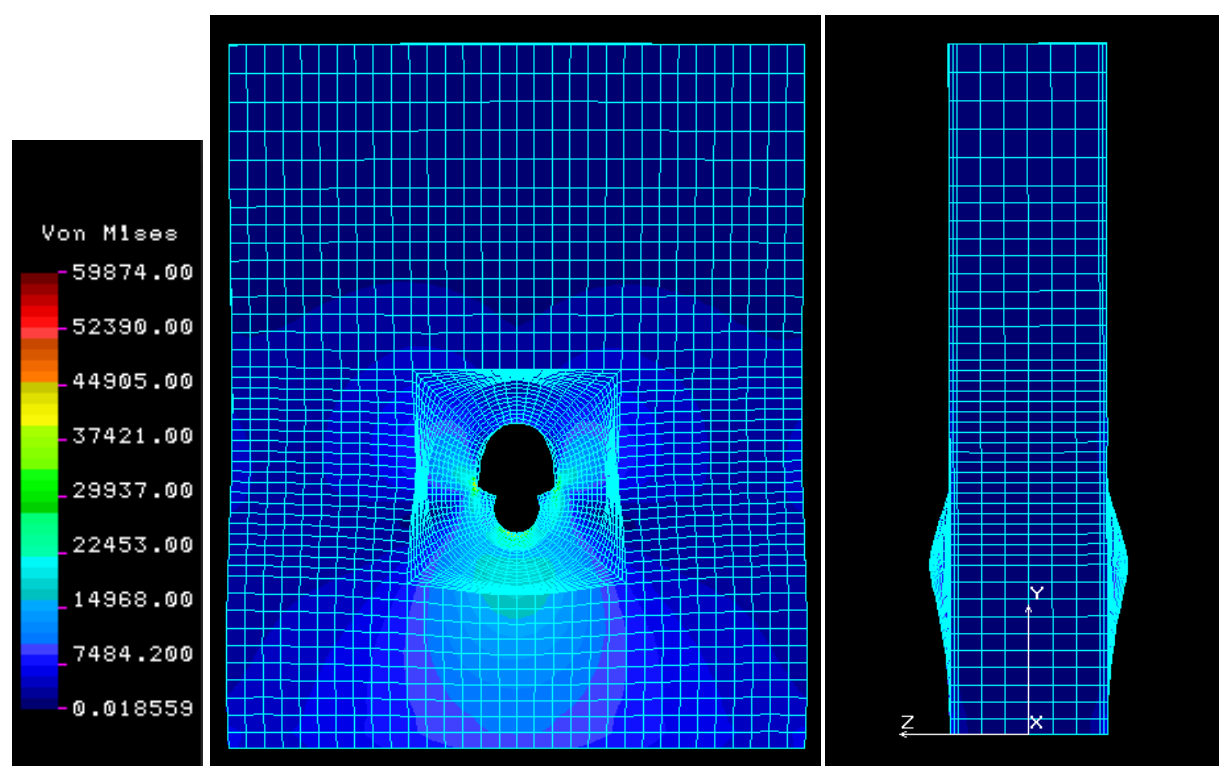

Figure 134: Stress response of FEM base model with bushing and pressure loading

The next analysis was the base model with the bushing and fastener for the control group.

The displacement response of the model can be seen in Figure 135. The stress response of the model can be seen in Figure 136. The displacement response of the model was similar to the model with the bushing. The model had more of a pronounced displacement under the bushing and the fastener. The model also had a similar bulge at the bottom of the hole appearing on the face sheet. An interesting observation that was seen in the model was that the addition of the fastener only decreased the maximum displacement seen on the specimen by half compared to the model with the bushing. The maximum displacement from the model was still an order of magnitude below the experimental results showing that the fastener and the bushing were still dissipating the force seen on the composite sandwich panel. 


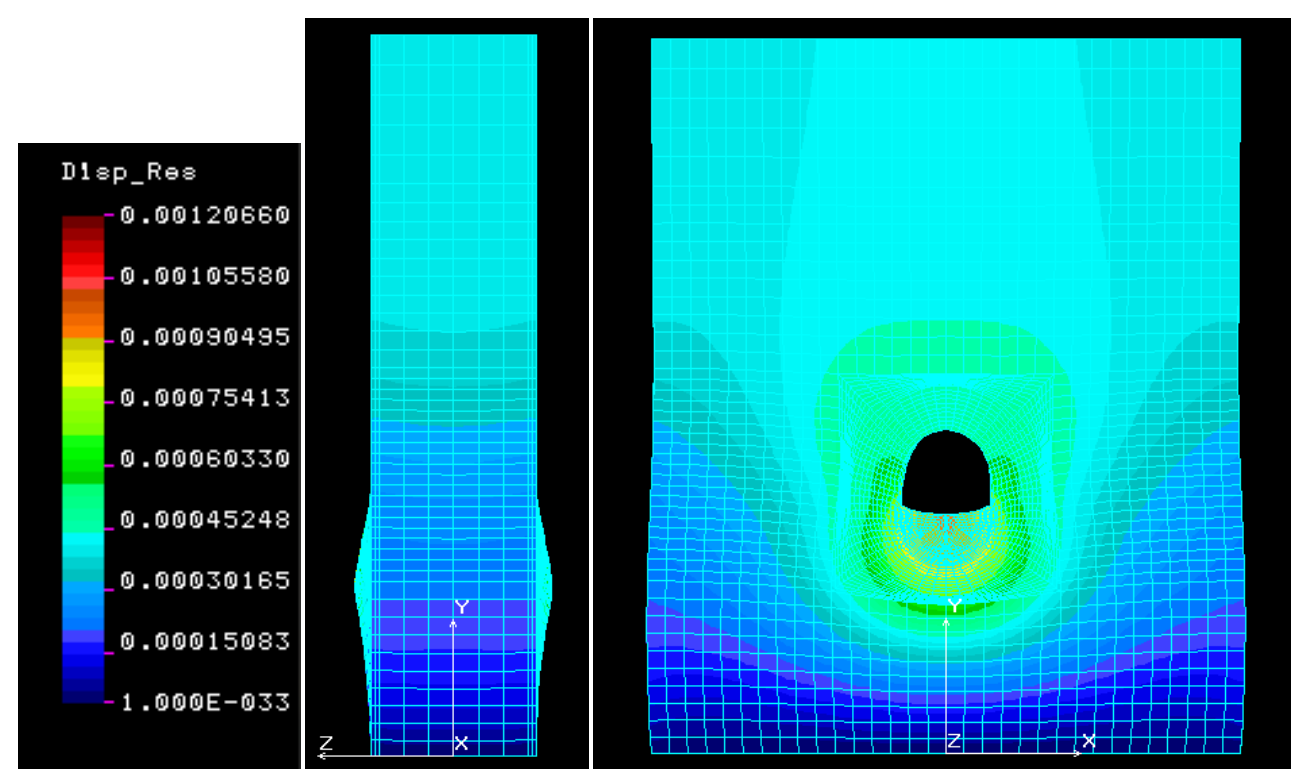

Figure 135: Displacement response of FEM base model with bushing, fastener and pressure loading

The stress distribution of the model with the bushing and fastener was similar to the bushing only model. The model still contained a more pronounced stress concentration as seen at the bottom of the hole than before. Instead of a circular shape, the stress concentration at the bottom of the hole stretched from the bushing all the way to the bottom of the model. This further backs up the assumption made earlier that the core was pushing along the face sheet at the bottom of the hole. With the addition of the bushing and the fastener, the stress concentration was actually able to push out onto the face sheet. 


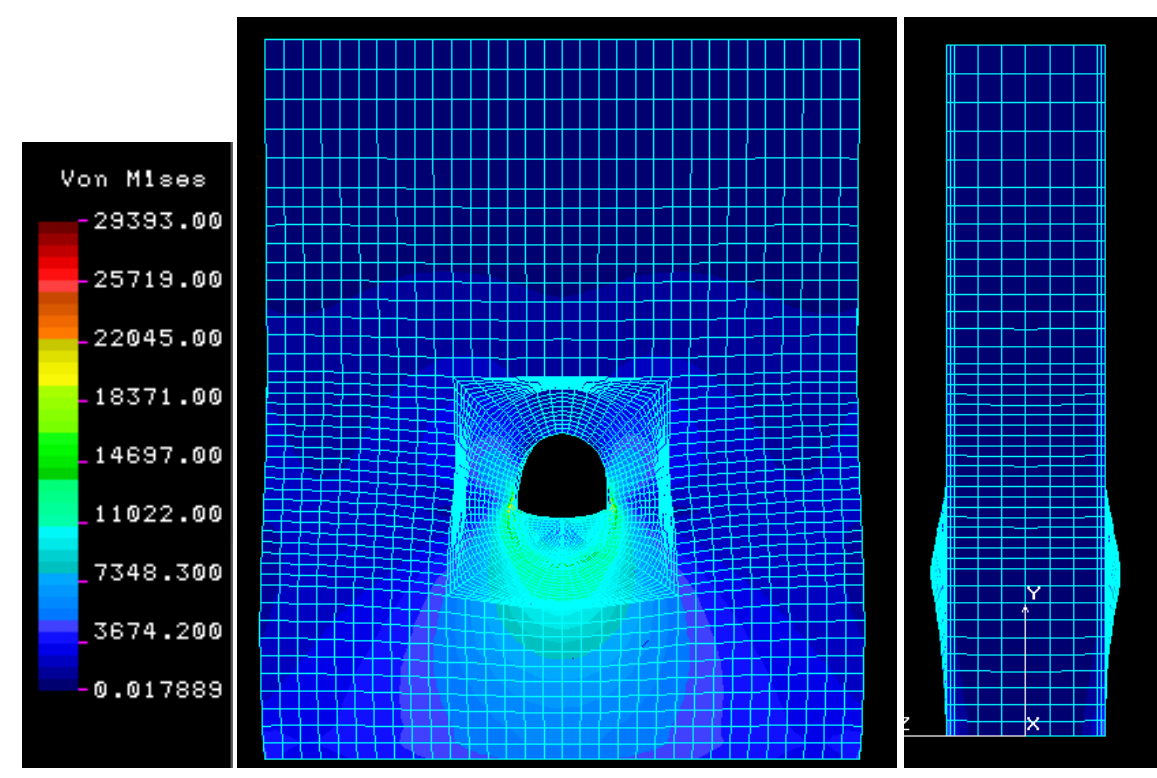

Figure 136: Stress response of FEM base model with bushing, fastener and pressure loading

The next analysis was the base model with DADs. The displacement response of the model can be seen in Figure 137. The stress response of the model can be seen in Figure 138. The base model for the DADs showed not that much of a displacement along the face sheet of the composite sandwich panel. The model did show that there was an elongation of the core. The increase in force due to the increase stiffness of the DADs seemed like it affected the core. Since the core was a lot weaker than the face sheet, the model showed that the core was being easily pushed down the panel, showed the elongation of the core in the model. The displacement contour bar shows that the maximum displacement was three times larger than the displacement observed from experimental testing. A little deformation was seen at the bottom of the hole, but was not as pronounced as the previous models. The little deformation could be due to the additional stiffness added from the DADs. This slightly resembled the failure results from the experimental testing. 


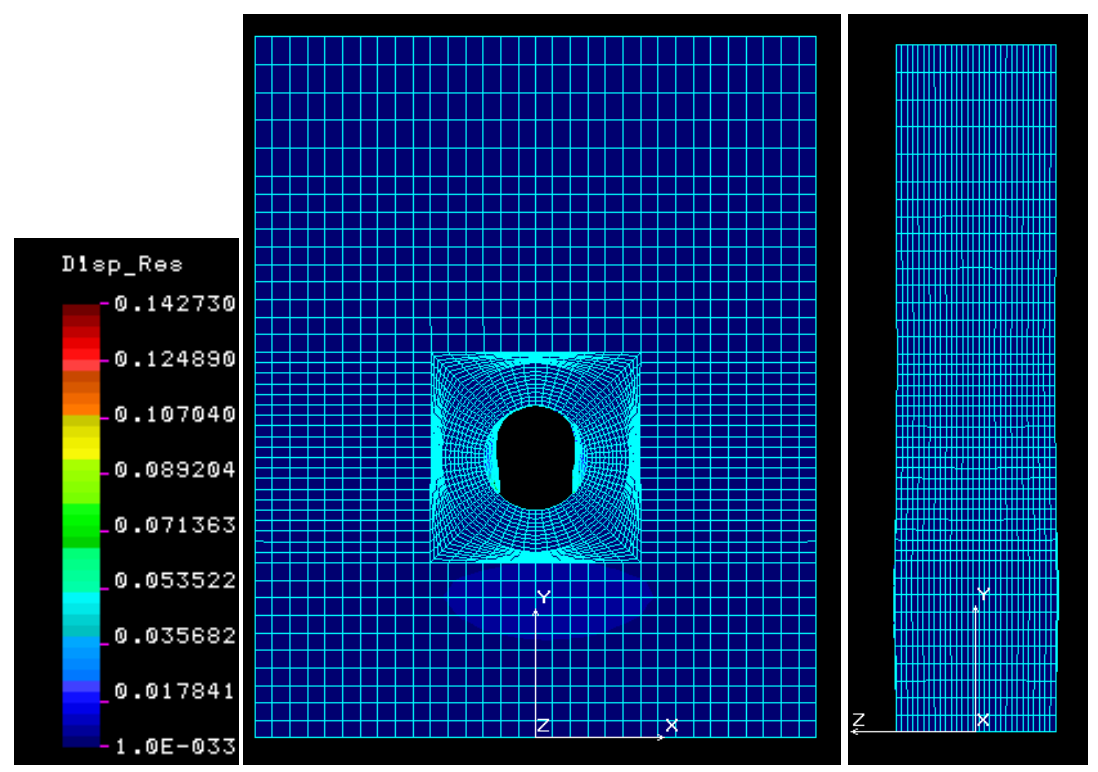

Figure 137: Displacement response of FEM DAD base model and pressure loading

The stress distribution of the model showed a far different distribution from the non-

DAD models. The first significant difference was that the model showed a stress concentration all along the DAD, running along the width of the model. The whole DAD showed that stresses were seen along it and that the stresses were higher where the $\mathrm{DAD}$ and the core meet. Another stress concentration was also seen at the bottom of the model. This could be a counter reaction from the boundary conditions placed on the bottom of the model. It can be mainly created from the core being pushed down more than the previous models creating a high stress concentration. 

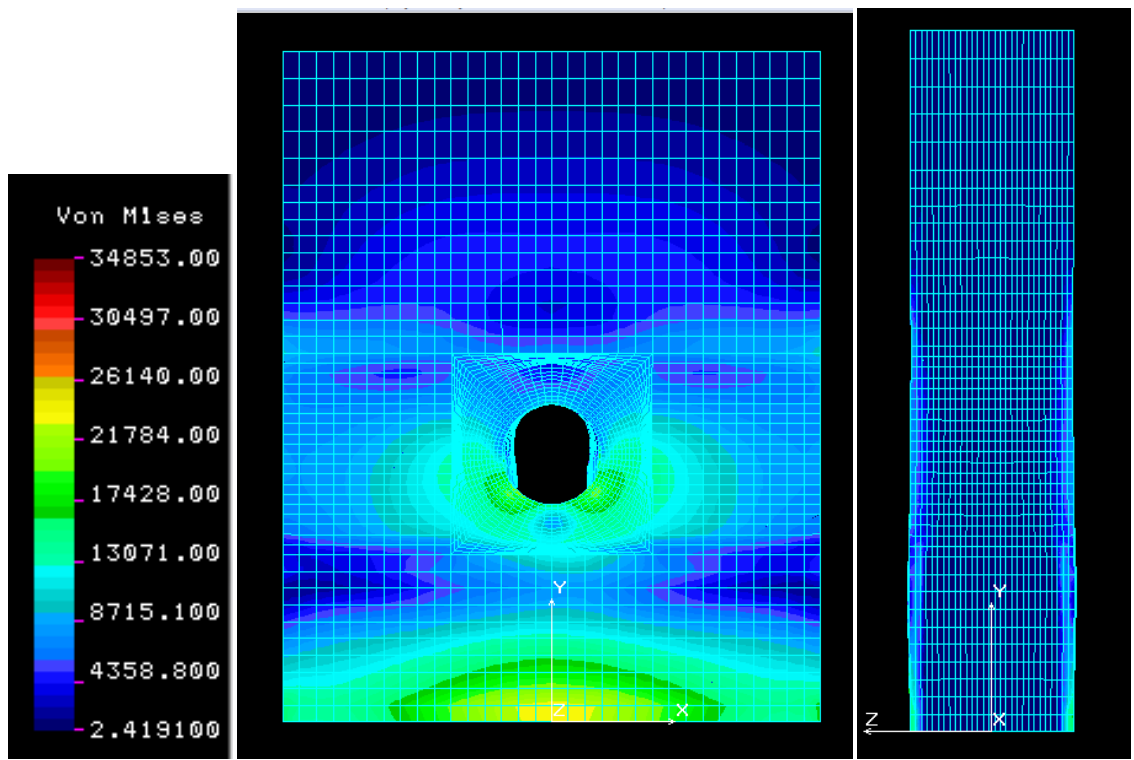

Figure 138: Stress response of FEM DAD base model and pressure loading

The next analysis was the base model with DADs and a bushing added to the model. The displacement response of the model can be seen in Figure 139. The stress response of the model can be seen in Figure 140. The displacement response showed a far different deformation from the base DAD model. The first significant difference was that the model had a more pronounced bulge at the bottom of the hole than all the other models. The side profile view showed that the model's deformation resembled a vase with the thickness of the model getting smaller at the hole and becoming larger at the base. The largest displacement was seen right below the model, creating a circular shape displacement field. The maximum displacement from the model was still an order of magnitude below the experimental results showing that the bushing was still dissipating the force seen on the composite sandwich panel. 


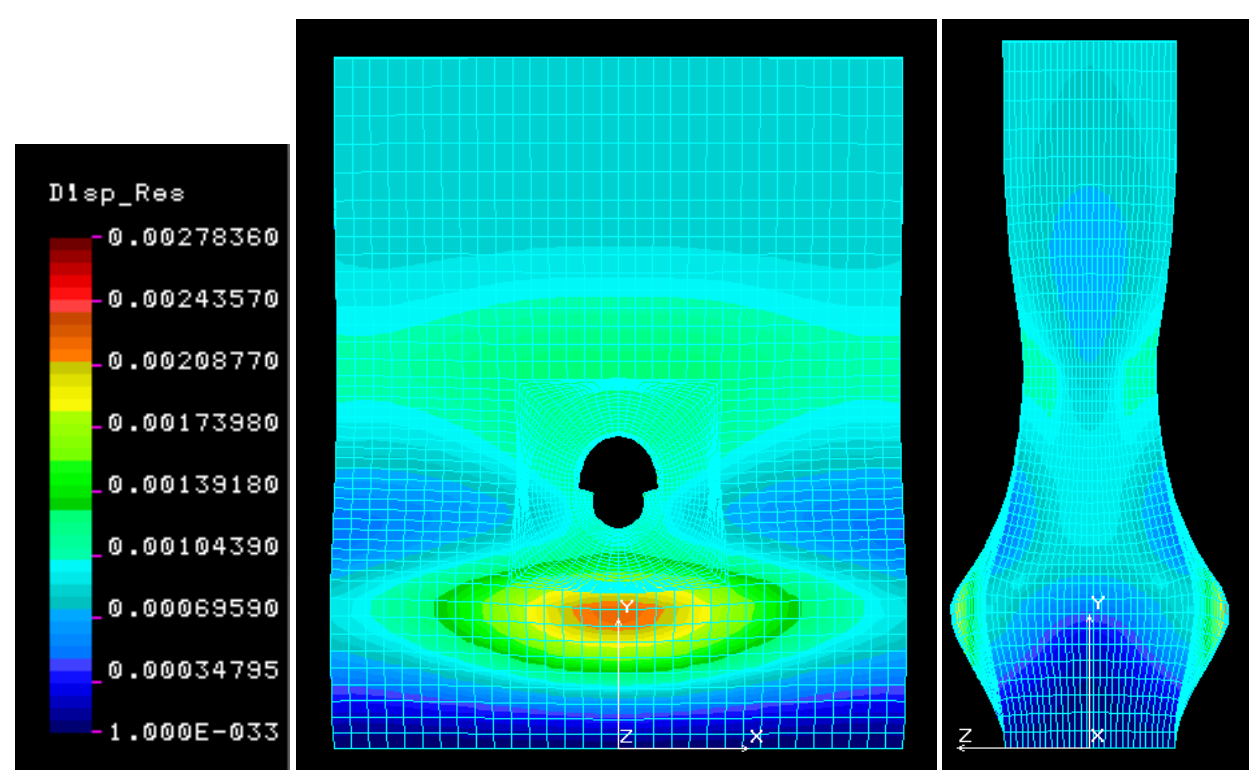

Figure 139: Displacement response of FEM DAD base model with bushing and pressure loading

The stress distribution of the model resembles more the stress distribution of the nonDAD models but greatly differs from the base DAD model. The stress distribution showed a stress concentration seen along the bottom of the model. Like before, this could be a counter reaction from the boundary conditions placed on the bottom of the model. It was mainly created from the core being pushed down more than the previous models creating a high stress concentration. The model also showed a stress concentration directly at the bottom of the hole but ends at the edge of the DAD. This was an interesting stress concentration because the figure showed that there was no stress transfer where the DAD and the core meet. This was far different from the previous model where stress concentrations were seen along the DAD. 


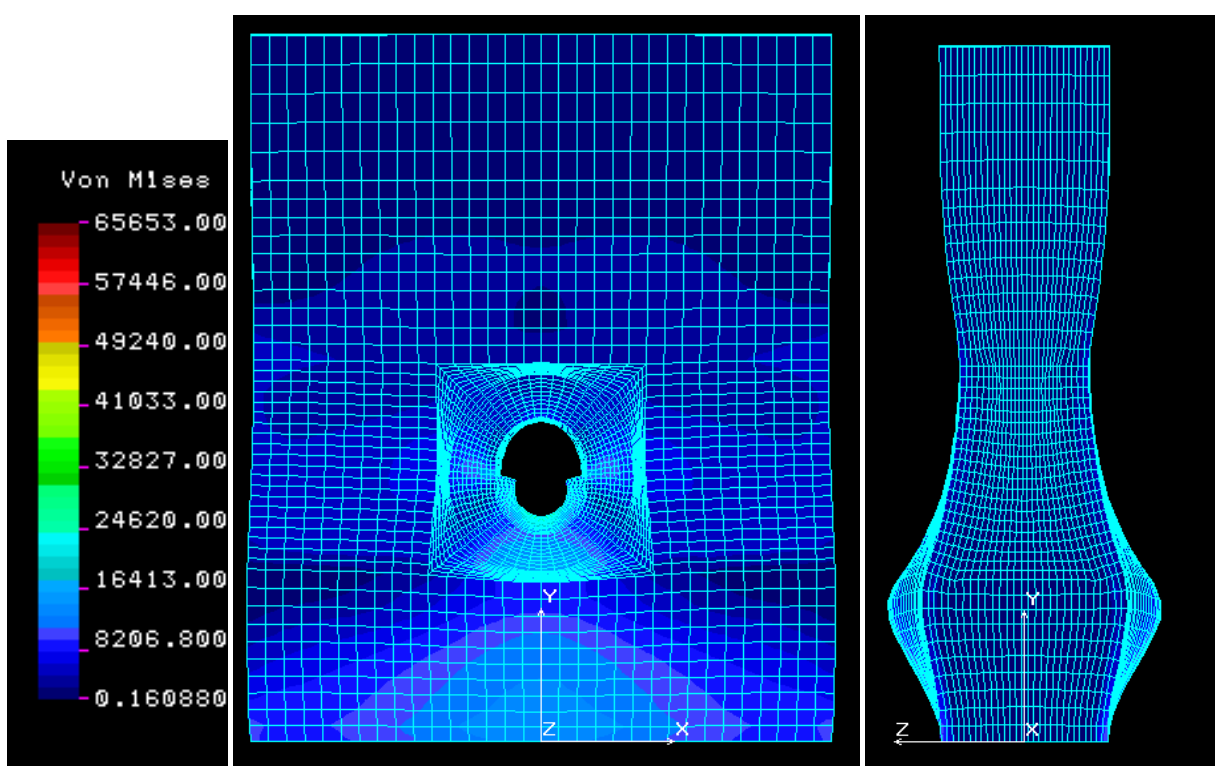

Figure 140: Stress response of FEM DAD base model with bushing and pressure loading

The next analysis was the base model with DADs with a bushing and a fastener added to the model. The displacement response of the model can be seen in Figure 141. The stress response of the model can be seen in Figure 142. The displacement response showed a similar response to the DAD base model with a bushing. The model had a similar bulge at the bottom of the hole as all the other models. The side profile view showed that the model's deformation resembles of a vase with the thickness of the model getting smaller at the hole and becoming larger at the base. The largest displacement was seen right below the model, creating a circular shape displacement field. The displacement distribution extended vertically around the hole of the composite sandwich panel making a distorted anvil shape. The maximum displacement from the model was still an order of magnitude below the experimental results showing that the bushing and fastener were still dissipating the force seen on the composite sandwich panel. This model shows a similar trend from the non-DAD models where the maximum displacement was only half the displacement seen on the DAD model with a bushing. 


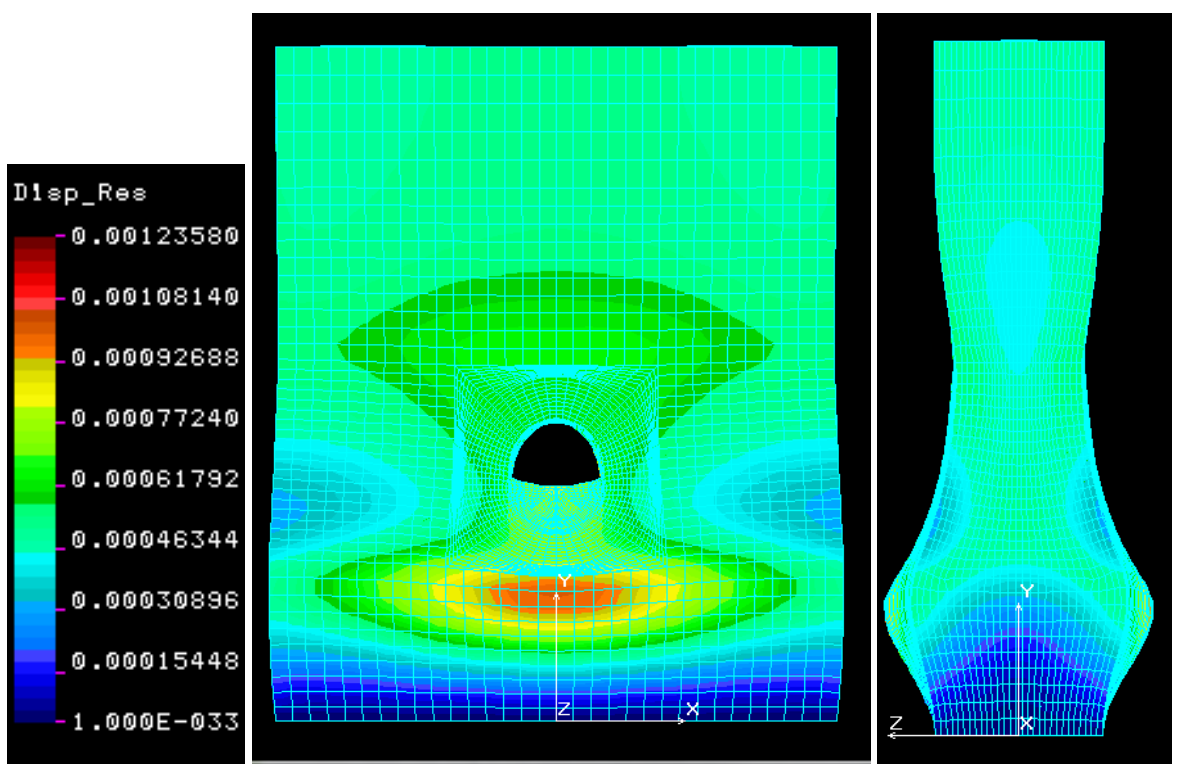

Figure 141: Displacement response of FEM DAD base model with bushing, fastener and pressure loading

The stress distribution of the model resembled a similar distribution to the bushing model. The stress distribution showed a stress concentration seen along the bottom of the model. Like before, this could be a counter reaction from the boundary conditions placed on the bottom of the model. It was mainly created from the core being pushed down more than the previous models creating a high stress concentration. The model also showed a stress concentration directly at the bottom of the hole and expanding slowly to the edge of the DAD. Instead of the previous model, the stress concentration did not stop but expands all the way to the bottom of the model. This shows that the model needed a better mesh between the fine mesh around the hole and the DAD and a coarse mesh at the bottom. A better transition in the mesh would give a smoother stress concentration at the bottom of the hole. 

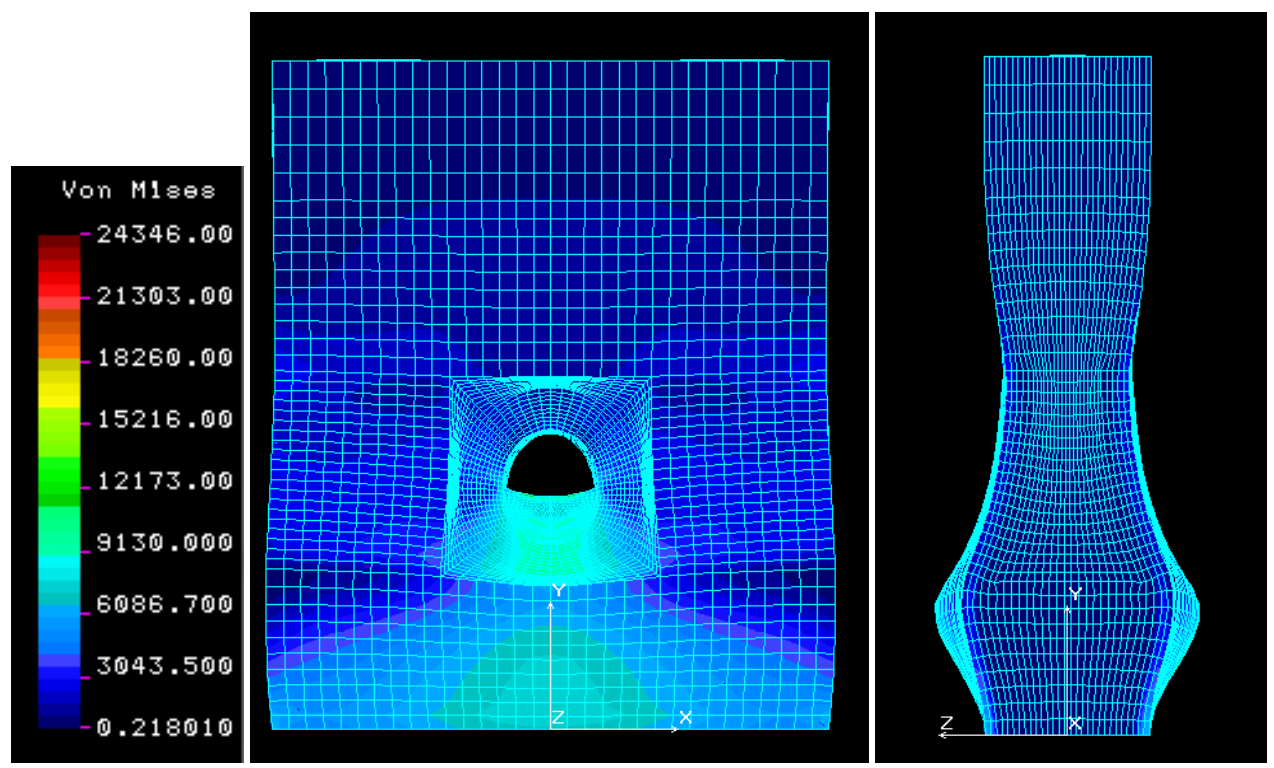

Figure 142: Stress response of FEM DAD base model with bushing, fastener and pressure loading

A summary of the displacement results for all six models with and without DADs can be seen in Table 31. The table shows that the base models for each group were in the same order of magnitude of numbers, while the base with the bushing and the base with the bushing and the fastener were one order of magnitude less than the base group. An interesting observation was that the base model with the bushing and the base model with the bushing and the fastener were the same for each group. This could be due to the dissipation seen in the metals where it contacted the composite sandwich panel. A more accurate loading must be applied for better results. The comparison between the FEM and the experimental results will be further discussed in 9.1 Comparison of maximum displacement.

Table 31: FEM displacement results with pressure loading

\begin{tabular}{|c|c|c|}
\cline { 3 - 3 } \multicolumn{2}{c|}{} & Extension \\
\cline { 2 - 3 } Control Group (No DAD) & (in) \\
\cline { 2 - 3 } & Base & 0.0335 \\
\cline { 2 - 3 } & Base/Bushing/Fastener & 0.0012 \\
\hline \multirow{2}{*}{ DAD Group } & Base & 0.1473 \\
\cline { 2 - 3 } & Base/Bushing & 0.0028 \\
\cline { 2 - 3 } & Base/Bushing/Fastener & 0.0012 \\
\hline
\end{tabular}


To obtain better results, a force loading was applied on the center line of the hole. The first analysis that was conducted was the base model with no DADs with a force loading. The displacement response of the model can be seen in Figure 143. The stress response of the model can be seen in Figure 144. The displacement model showed that the displacement on the model was on an order of magnitude of seven, being nine whole orders above the experimental results. The displacement distribution also showed that parts of the core and face sheet have complete fractured and were hanging over the hole. This was totally unexpected and something happened during the analysis that caused a complete failure of the specimen. This was mainly due to the loading applied on the specimen. Instead of a pressure distribution which made sure that the yield loading was evenly distributed along the surface, the yield load was applied to each node along the center line. The displacement and stress distribution showed that there were no effects to the core or the face sheet. From the previous models, there was bulge present at the bottom of the hole or the core being distorted. The results for this case were omitted for analysis due to the obscure and unexpected results.
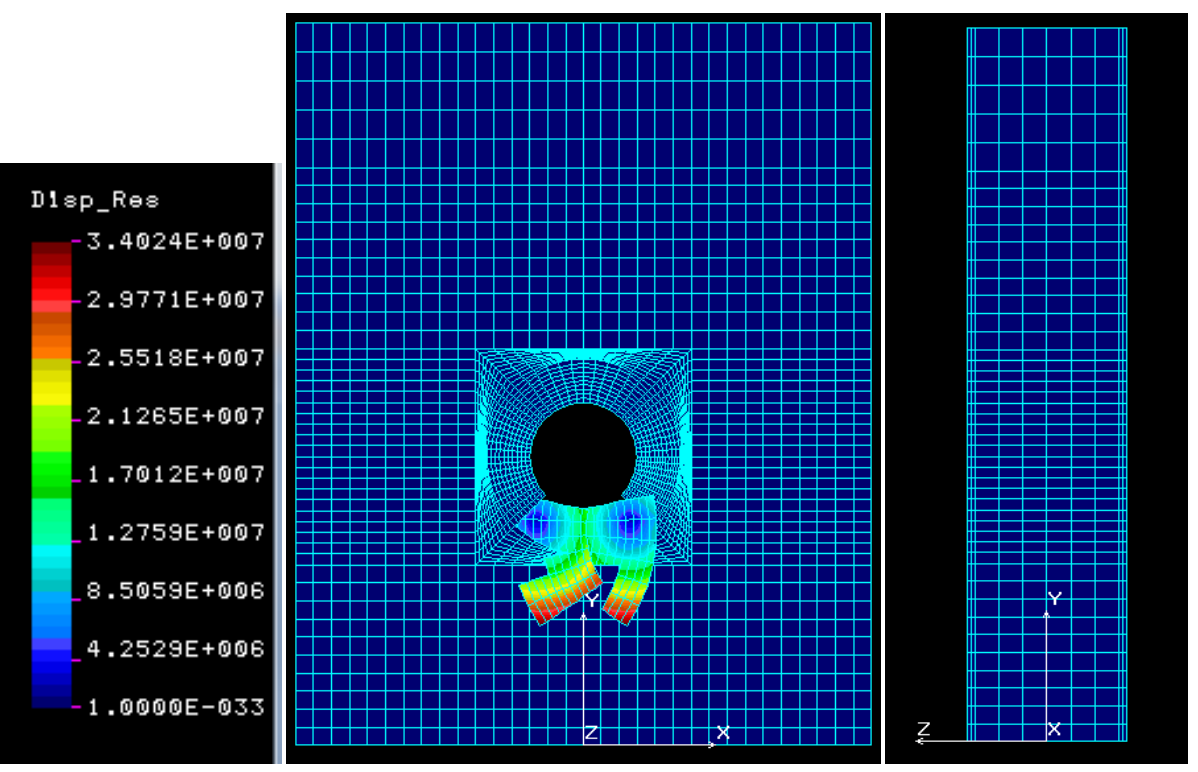

Figure 143: Displacement response of FEM base model with force loading 
The stress distribution shows that there were not that many stress concentrations seen on the face sheet of the composite sandwich panel. The stresses seen on the model could be coming more from the core than the face sheet. Since a direct force was applied on the core, the core must be deforming within the panel causing the failure in the model.

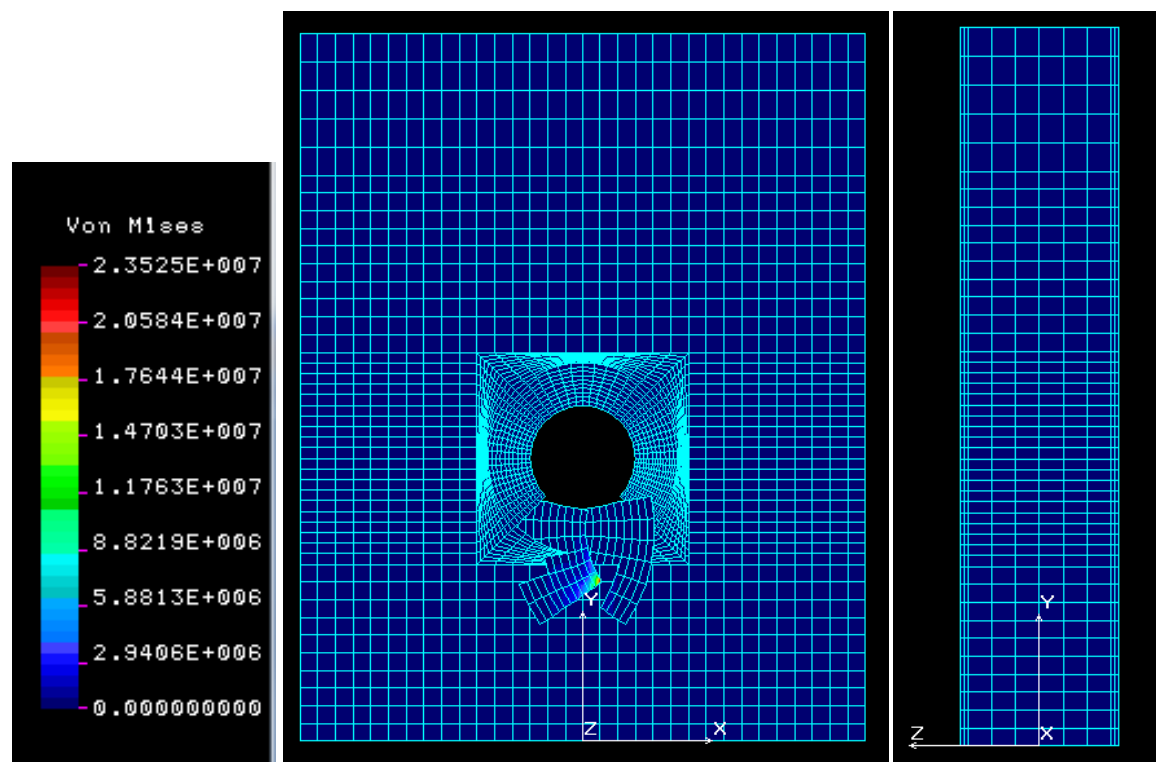

Figure 144: Stress response of base model with force loading

The next analysis for the force loading was the base model with the bushing. The displacement response of the model can be seen in Figure 145. The stress response of the model can be seen in Figure 146. The displacement model resembled more the base model with bushing with pressure loading. Both displacement models had similar contour curves on the model, showing that regardless of the loading, the bushing applied a similar distribution on the specimen. The main difference between the two models was that there was a sharp indent on the bushing caused by the loading on the centerline of the hole. The model also had a similar bulge at the bottom of the hole. The great advantage to this model was that the maximum displacement of the specimen was in the same order of magnitude as the experimental results. The displacement distribution also showed that the maximum displacement of the model occurred in the core. 


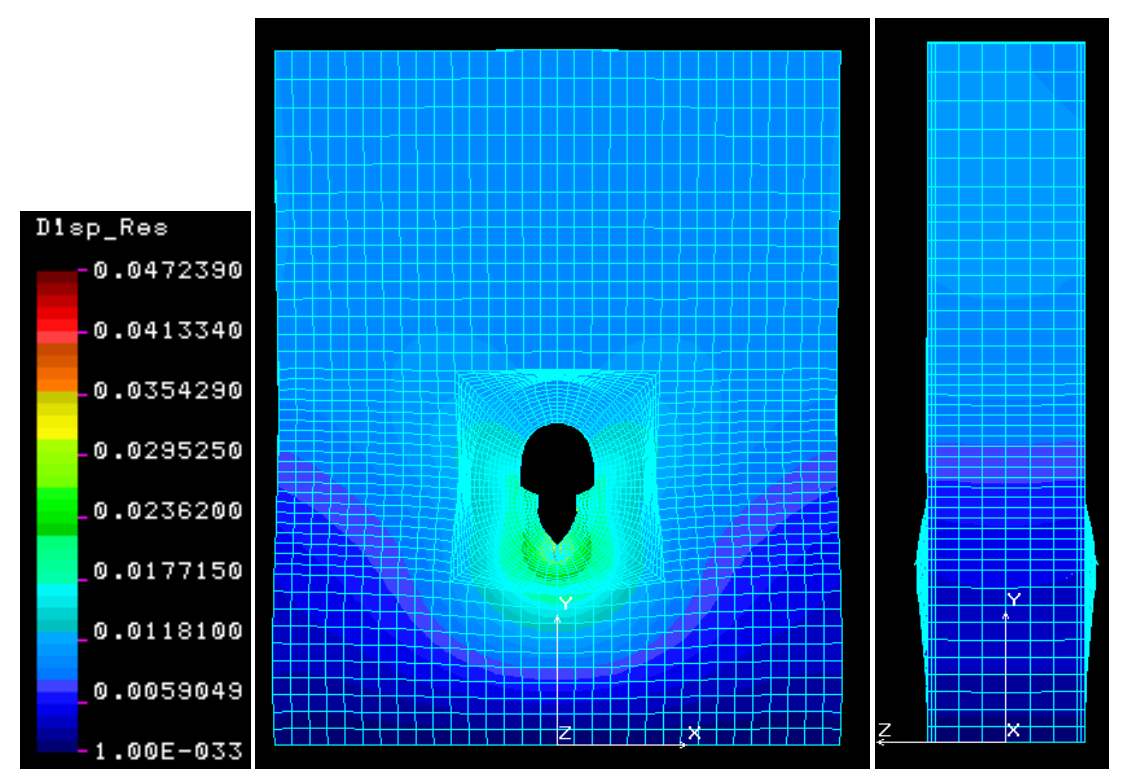

Figure 145: Displacement response of FEM base model with bushing and force loading

The stress distribution of the specimen showed that the use of force loading on the model did not create any stress concentrations on the face sheet. It looked like most of the stress concentrations occured on the foam at the hole. The model shows that there was an increase in stress around the hole compared to the rest of the outer surface of the model.
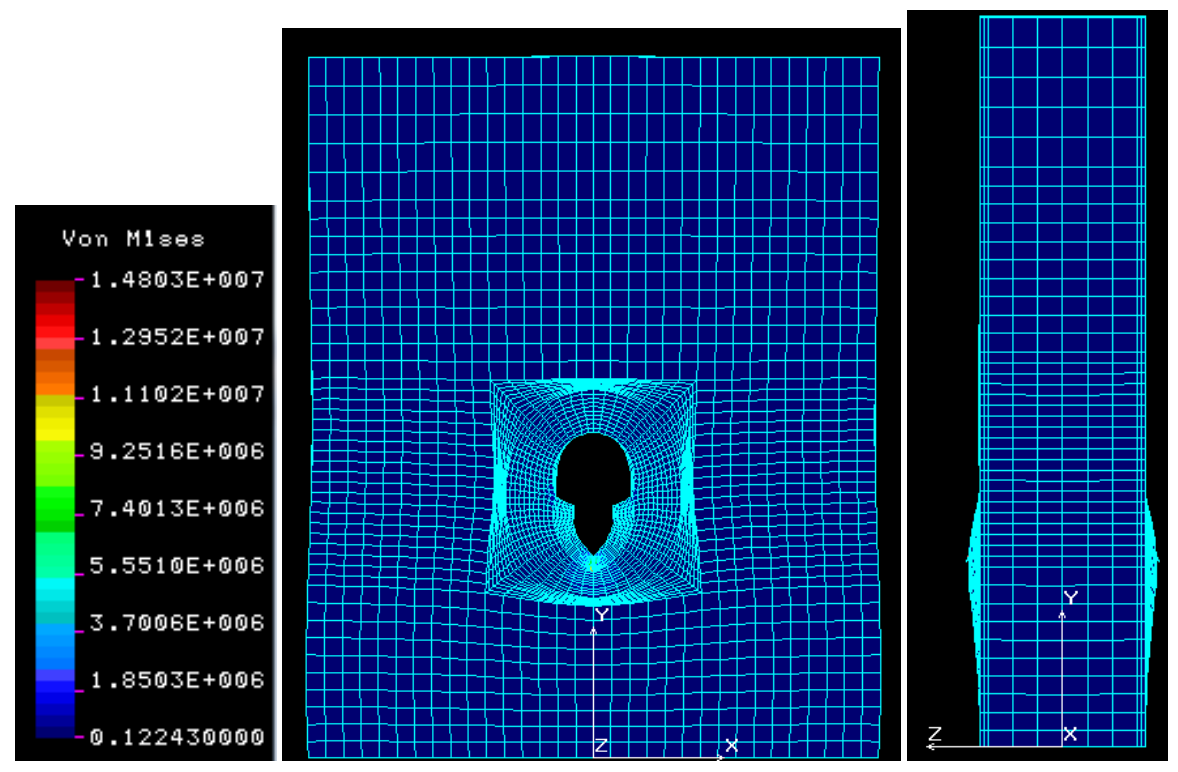

Figure 146: Stress response of FEM base model with bushing and force loading 
The next analysis for the force loading was the base model with the bushing and the fastener. The displacement response of the model can be seen in Figure 147. The stress response of the model can be seen in Figure 148. The displacement model resembled more the base model with the bushing and the fastener and pressure loading. Both models had similar contour curves on the model, showing that regardless of the loading, the bushing and fastener would applied a similar distribution on the specimen. The main difference between the two models was that there was a sharp indent on the bushing caused by the loading on the centerline of the hole. The model also had a similar bulge at the bottom of the hole. The bulge was less pronounced than previous models. The great advantage to this model was that the maximum displacement of the specimen was in the same order of magnitude as the experimental results.

The stress distribution of the specimen showed that the use of force loading on the model did not create any stress concentrations on the face sheet. It looked like most of the stress concentrations occurred on the foam at the hole. The model showed that there was an increase in stress around the hole compared to the rest of the outer surface of the model.

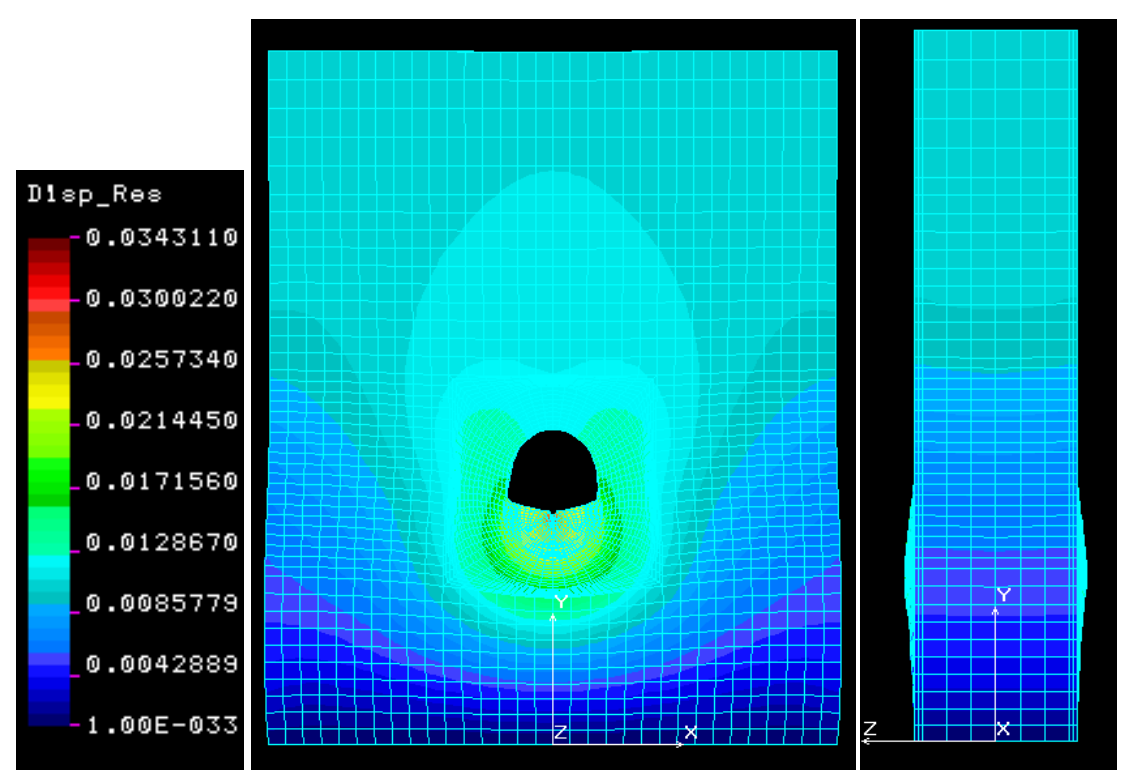

Figure 147: Displacement response of FEM base model with bushing, fastener and force loading 


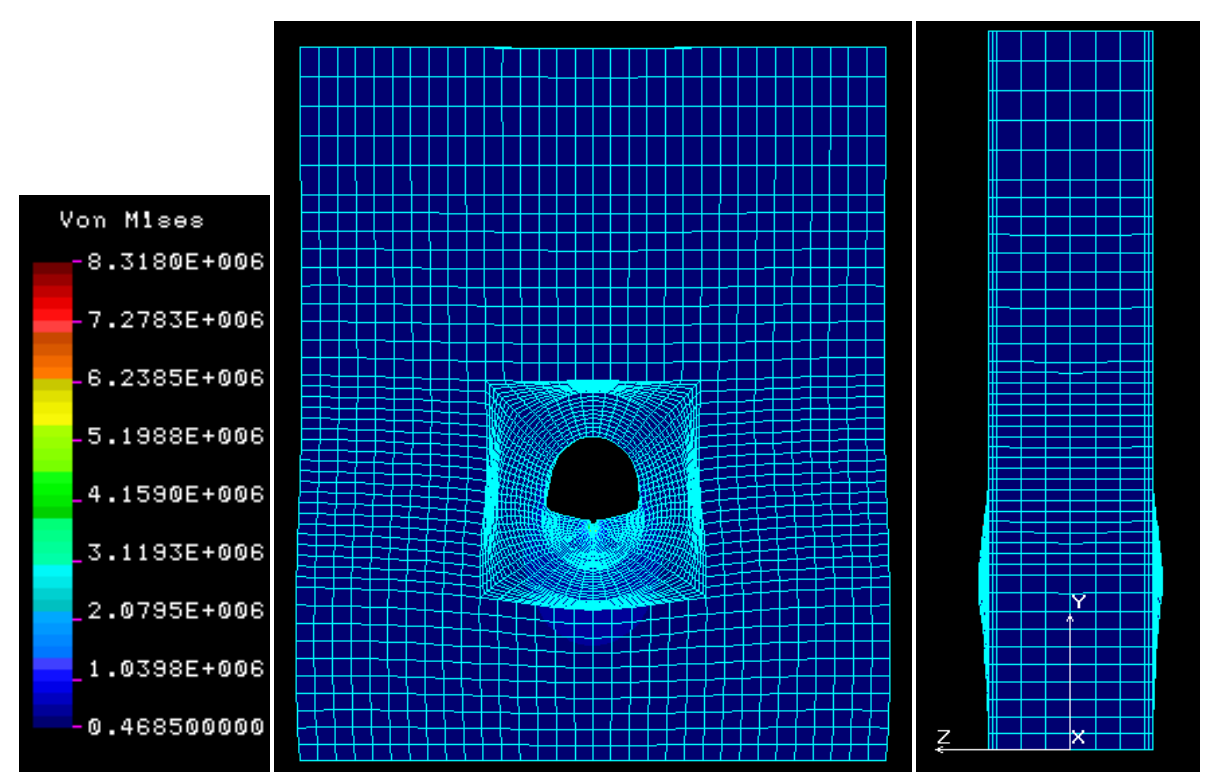

Figure 148: Stress response of FEM base model with bushing, fastener and force loading

The next analysis for the force loading was the base model with DADs. The displacement response of the model can be seen in Figure 149. The stress response of the model can be seen in Figure 150. Like the base model with no DADs with force loading, there was no significant displacement seen on the outer surface of the specimen. There was no bulge or any deformation seen on the model. This could be due to the addition of the DADS adding infinite stiffness to the model. The lack of deformation or displacement could be due to only having the loading on the centerline of the bottom hole surfaces. The maximum displacement contour bar showed that the maximum displacement seen on the specimen was three orders of magnitude higher than the experimental testing. This showed that the loading was affecting the model in an unusual and unexpected way. The results for this model were omitted for analysis due to the high displacement value and the lack of any distortion on the model. 


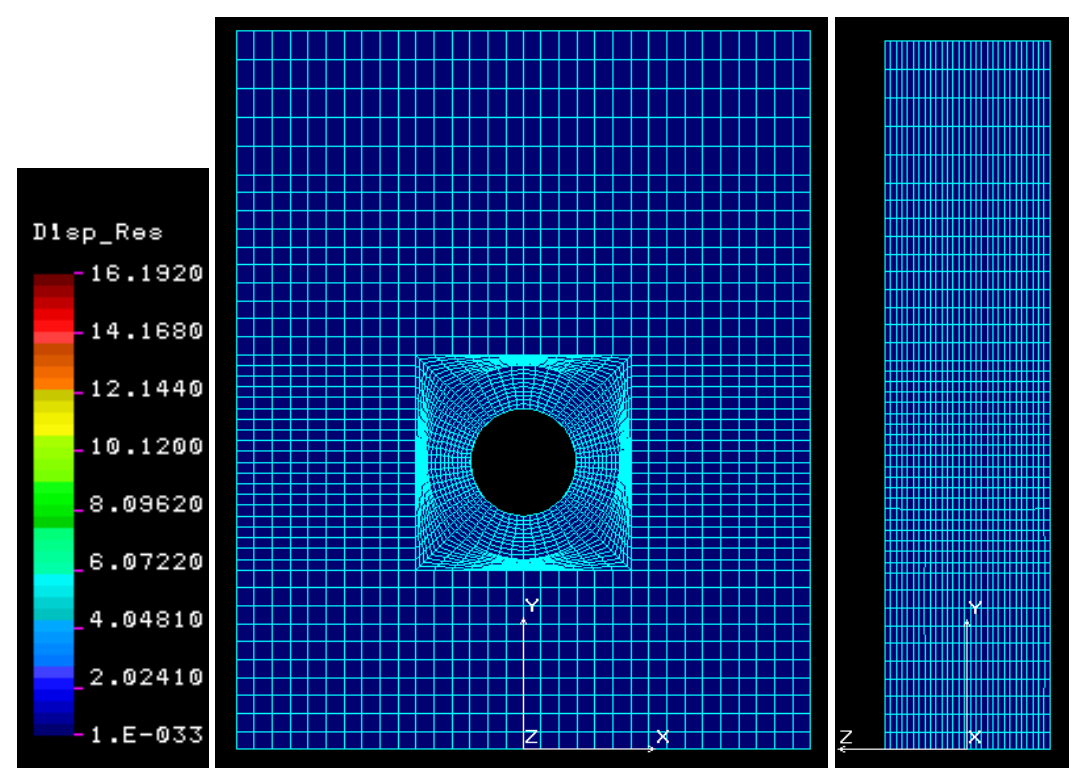

Figure 149: Displacement response of FEM DAD base model with force loading

The stress distribution of the model showed no high stress concentrations on the model.

The distribution did show an increase stress at the bottom of the model and around the hole. The slight increase in stress resembles the shape of a mushroom cloud. This increase in stress could be due to the counter forces from the boundary conditions placed on the bottom of the model.

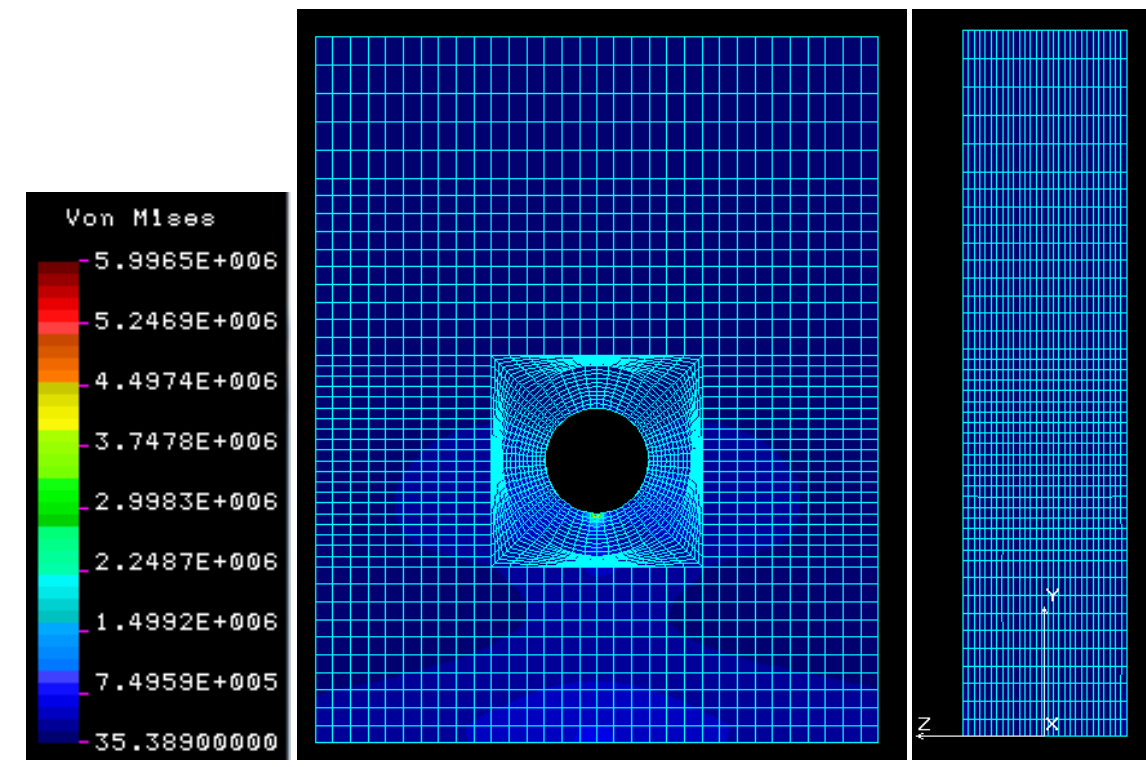

Figure 150: Stress response of FEM DAD base model with force loading 
The next analysis with the force loading was the base model with DADs with a bushing and a fastener added to the model. The displacement response of the model can be seen in Figure 151. The stress response of the model can be seen in Figure 152. The displacement response showed a similar response to the DAD base model with a bushing and force loading. The model had a similar bulge at the bottom of the hole as all the other models. The side profile view showed that the model's deformation resembles of a vase with the thickness of the model getting smaller at the hole and becoming larger at the base. The largest displacement was seen right below the model, creating a circular shape displacement field. The displacement distribution extended vertically around the hole of the composite sandwich panel making a distorted anvil shape. The maximum displacement from the model was an order of magnitude above the experimental results showing that the bushing and fastener was surprisingly not dissipating the loading compared to the previous loading analysis with this model.

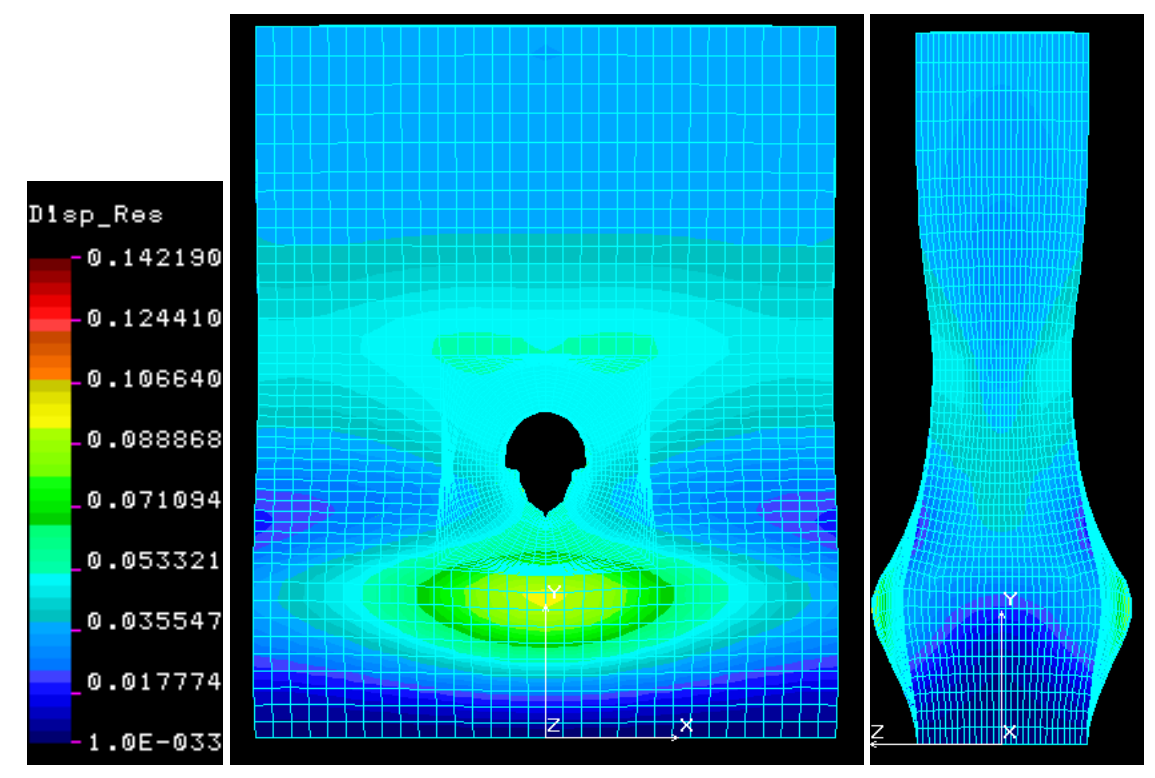

Figure 151: Displacement response of FEM DAD base model with bushing and force loading

The stress distribution of the models showed more of a stress concentration than previous models. The model had an increase stress concentration seen at the bottom of the panel. The 
slight increase in stress had a triangular shape with the tip of the triangle near where the DAD and the core meet.

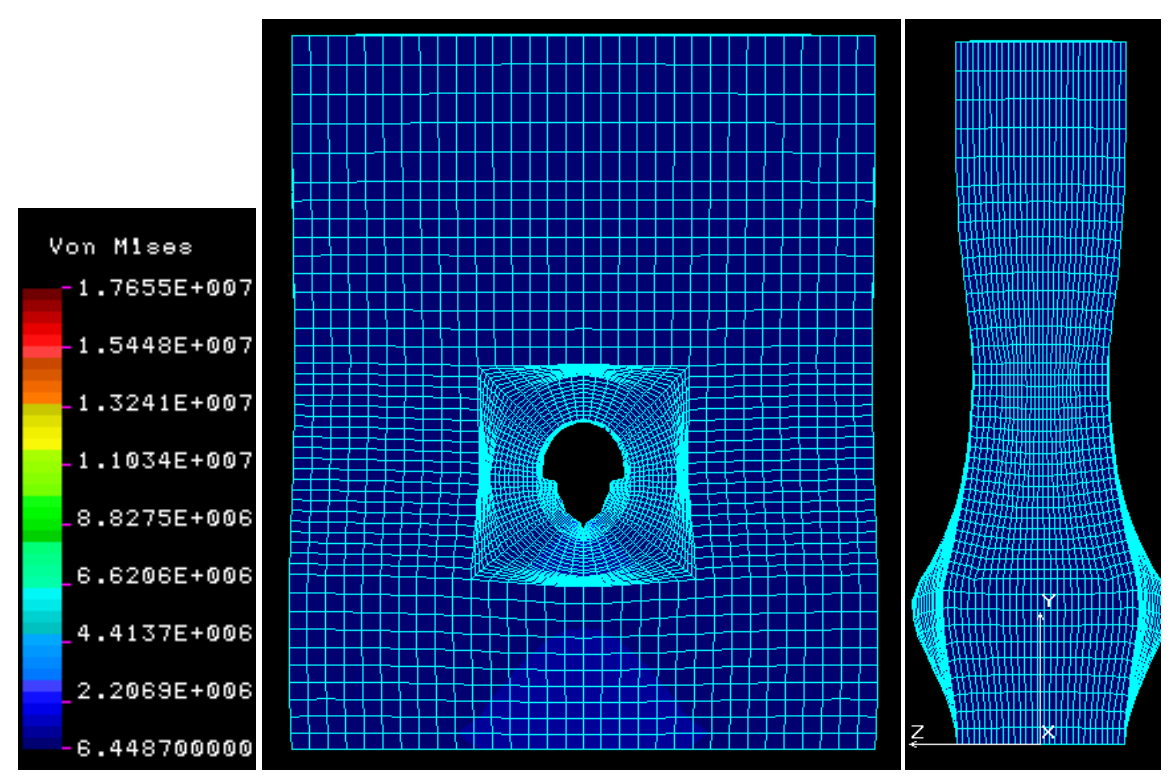

Figure 152: Stress response of FEM DAD base model with bushing and force loading

The last analysis with the force loading was the base model with DADs with a bushing and a fastener added to the model. The displacement response of the model can be seen in Figure 153. The stress response of the model can be seen in Figure 154. The displacement response showed a similar response to the DAD base model with a bushing. The model had a similar bulge at the bottom of the hole as all the other models. The side profile view showed that the model's deformation resembles a vase with the thickness of the model gets smaller at the hole and becoming larger at the base. The largest displacement was seen right below the model, creating a circular shape displacement field. The displacement distribution extended vertically around the hole of the composite sandwich panel making a distorted anvil shape. The maximum displacement from the model was the same order of magnitude of the experimental results. 


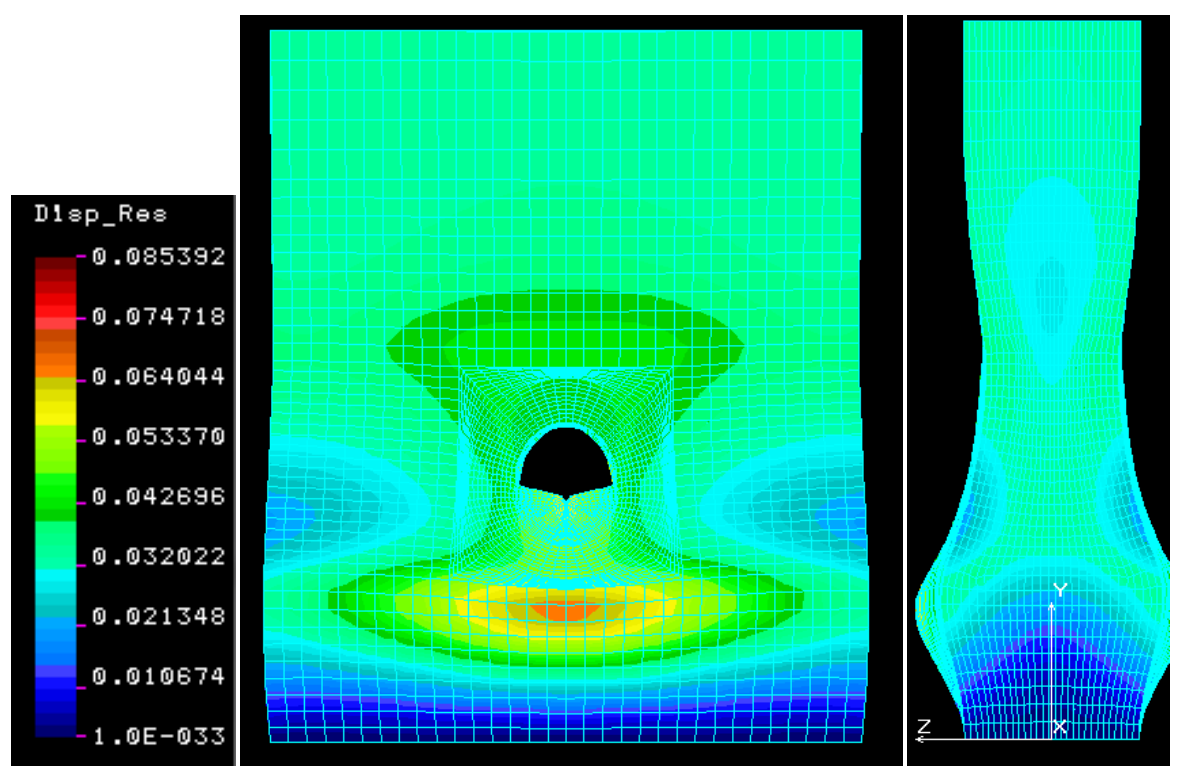

Figure 153: Displacement response of FEM DAD base model with bushing, fastener and force loading

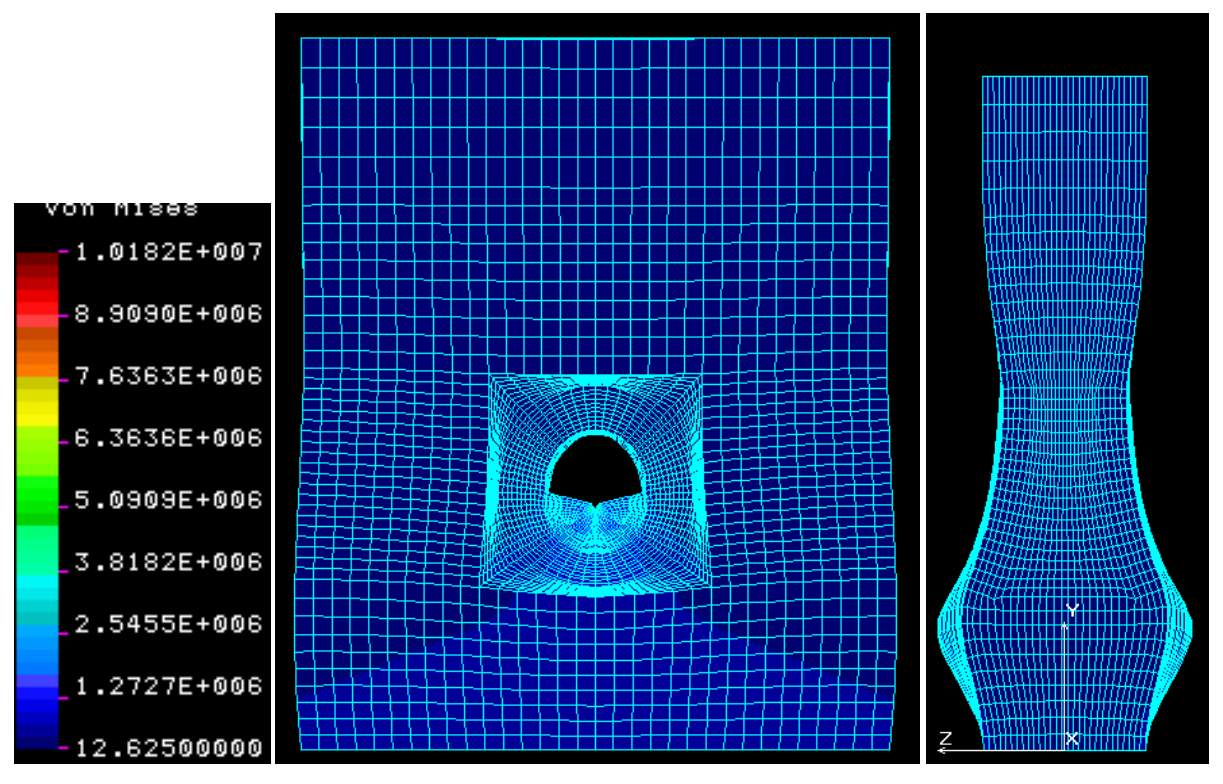

Figure 154: Stress response of FEM DAD base model with bushing, fastener and force loading

A summary of the displacement results for all six models with and without DADs can be seen in Table 32. The tables showed that the base models for each group were omitted having too high displacements due to the unexpected and obscure results. The use of the force loading made the results for both groups more in line with the experimental results. The control groups, models without DADs, were pretty close to one another showing that the addition of the fastener 
dissipated the force seen on the panel compared to the bushing alone. The DAD group showed the same trend by having a smaller displacement with the addition of the fastener. The comparison between the FEM and the experimental results will be further discussed in 9.1 Comparison of maximum displacement.

Table 32: FEM displacement results with force loading

\begin{tabular}{|c|c|c|}
\cline { 3 - 3 } \multicolumn{2}{|c|}{} & Extension \\
\cline { 2 - 3 } Control Group (No DAD) & Bin) \\
\cline { 2 - 3 } & Base/Bushing & N/A \\
\cline { 2 - 3 } & Base/Bushing/Fastener & 0.0472 \\
\hline \multirow{3}{*}{ DAD Group } & Base & N/A \\
\cline { 2 - 3 } & Base/Bushing & 0.1422 \\
\cline { 2 - 3 } & Base/Bushing/Fastener & 0.0854 \\
\hline
\end{tabular}




\section{Comparison between Theoretical, Experimental and FEM}

\subsection{Comparison of maximum displacement}

A comparison of the maximum displacement or extension of the composite sandwich panel between the experimental testing and the FEMs was conducted. The comparison of results can be seen in Table 33. The table shows the results from both the pressure and force loading with and without DAD for the FEM models. The results highlighted with yellow represent the most accurate in extension/displacement for the FEM model compared to its respective group. For the control group, non DAD models, the base model with a bushing and force loading was the most accurate by under predicting the experimental extension by $14.1 \%$ by having an extension of 0.0472 in. For the DAD group, the base model with a bushing, a fastener, and force loading was the most accurate by over predicting the experimental extension by $43.0 \%$ by having an extension of 0.0854 in.

Table 33: Comparison of maximum displacement between Experimental and FEM Results

\begin{tabular}{|c|c|c|c|c|}
\hline & & & Extension & \% Difference \\
\hline & & & (in) & $(-)$ \\
\hline \multirow{8}{*}{$\begin{array}{l}\text { Pressure } \\
\text { Loading }\end{array}$} & \multirow{4}{*}{$\begin{array}{l}\text { Control } \\
\text { Group (No } \\
\text { DAD) }\end{array}$} & Experimental & 0.0550 & - \\
\hline & & Base & 0.0335 & $-39.1 \%$ \\
\hline & & Base/Bushing & 0.0028 & $-94.9 \%$ \\
\hline & & Base/Bushing/Fastener & 0.0012 & $-97.8 \%$ \\
\hline & \multirow{4}{*}{ DAD Group } & Experimental & 0.0597 & - \\
\hline & & Base & 0.1473 & $146.7 \%$ \\
\hline & & Base/Bushing & 0.0028 & $-95.3 \%$ \\
\hline & & Base/Bushing/Fastener & 0.0012 & $-97.9 \%$ \\
\hline \multirow{8}{*}{ Force Loading } & \multirow{4}{*}{$\begin{array}{l}\text { Control } \\
\text { Group (No } \\
\text { DAD) }\end{array}$} & Experimental & 0.0550 & - \\
\hline & & Base & N/A & - \\
\hline & & Base/Bushing & 0.0472 & $-14.1 \%$ \\
\hline & & Base/Bushing/Fastener & 0.0343 & $-37.6 \%$ \\
\hline & \multirow{4}{*}{ DAD Group } & Experimental & 0.0597 & - \\
\hline & & Base & N/A & - \\
\hline & & Base/Bushing & 0.1422 & $138.2 \%$ \\
\hline & & Base/Bushing/Fastener & 0.0854 & $43.0 \%$ \\
\hline
\end{tabular}


Some interesting trends from the table was that for both groups with pressure loading, the base model with a bushing and the base model with both the bushing and the fastener were a complete order of magnitude below the experimental results. This could be mainly due to how the loading was transferred to the composite sandwich panel by the metal inserts. On the other hand, the base models for both groups with pressure loading were in the same order of magnitude with the experimental results.

Another interesting trend from the table was that for both groups with force loading, the extension decreased as the models become more complex. Adding a fastener to both groups’ models decreased the extension. This reinforced the assumption that adding the bushing or the fastener to the model would dissipate the load seen by the composite sandwich panel. The last interesting trend was that for the force loading models, the control group under predicted the experimental results and the DAD group over predicted the experimental results. This could be due to the addition of the DADs and showed that another type of loading must be applied to see if better results can be obtained for that group.

Changing the loading on the specimen from a pressure loading to a force loading along the centerline of the bottom surface of the hole significantly affected the overall extension of the specimen. It moved the results from being one complete order of magnitude below the experimental results to within the same order of magnitude. The change to the new loading also gave better results, making the models ideal for future works.

\subsection{Comparison of load/extension curve and Elastic Modulus}

A comparison of the load versus extension curve and the elastic modulus for the theoretical, experimental, and FEM results was conducted. The first comparison was the control group without any DADs for the load versus extension curve; this can be seen in Figure 155. The figure showed five curves: the theoretical curve, the experimental curve, the FEM with force loading on the base model with a bushing, the FEM with force loading on the base model with a 
bushing and a fastener, and the FEM with pressure loading on the base model. The three FEM were plotted because those were the only models that had the same order of magnitude to the experimental or theoretical results. The FEM curves were created changing the loading of the model to find its respective displacement. Five data points were taken for each FEM model to create the curve. The theoretical curve was obtained using the force spring equation and solved for displacement from stiffness and the yield force. The experimental curve was plotted using the test specimen that had the closest to the average elastic modulus from that test group.

The FEM results have a linear curve that end at the yield load and its respective yield extension because the FEM software assumes that the model was running in the elastic region and assuming a linear failure model. A more advanced analysis or software must be used to have a more non-linear curve.

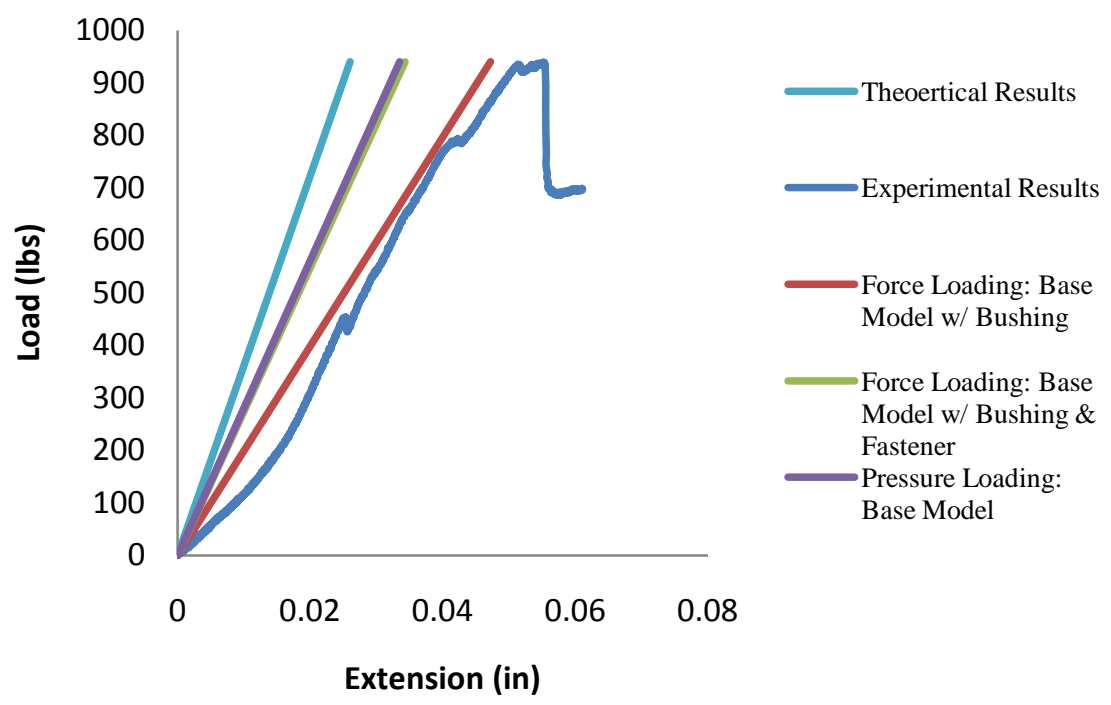

Figure 155: Load/Extension Comparison for Control Group

From the figure, it shows that the force loading on the base model with bushing and the pressure loading on the base model had similar slopes to the experimental results. The force loading on the base model with a bushing had the closest yield extension to the experimental results, but it had more of a gradual slope than the experimental results. The figure shows that the 
steeper the slope of the curve, the lower the yield extension from the experimental results. The elastic modulus of the curves can be seen in Table 34. The table showed that the theoretical elastic modulus of the composite sandwich panel was the highest. The elastic modulus was calculated by taking the slopes from the load versus extension curves, the slope was the stiffness (k), and was converted to elastic modulus by the geometry of the ideal test specimen. The FEM with force loading on the base model with a bushing had the lowest elastic modulus but was the closest in maximum displacement compared to the experimental results. The FEM with force loading on the base model with a bushing and a fastener and the FEM with pressure loading on the base model had similar elastic modulus and extension.

Table 34: Elastic Modulus Comparison for Control Group

\begin{tabular}{|c|c|c|c|}
\cline { 2 - 4 } \multicolumn{1}{c|}{} & $\begin{array}{c}\text { Elastic } \\
\text { Modulus }\end{array}$ & $\begin{array}{c}\text { \% Difference } \\
\text { from Theoretical }\end{array}$ & $\begin{array}{c}\text { \% Difference from } \\
\text { Experimental }\end{array}$ \\
\cline { 2 - 4 } & $(\mathrm{psi})$ & $(-)$ & - \\
\hline Theoretical Results & 62,650 & - & - \\
\hline Experimental Results & 45,174 & $-27.9 \%$ & $-23.5 \%$ \\
\hline $\begin{array}{c}\text { Force Loading: Base } \\
\text { Model w/ Bushing }\end{array}$ & 34,573 & $-44.8 \%$ & $5.3 \%$ \\
\hline $\begin{array}{c}\text { Force Loading: Base } \\
\text { Model w/ Bushing \& } \\
\text { Fastener }\end{array}$ & 47,578 & $-24.1 \%$ & $7.8 \%$ \\
\hline $\begin{array}{c}\text { Pressure Loading: } \\
\text { Base Model }\end{array}$ & 48,715 & $-22.2 \%$ & - \\
\hline
\end{tabular}

The first comparison was the control group with DADs for the load versus extension curve; this can be seen in Figure 156. The figure showed four curves: the theoretical curve, the experimental curve, the FEM with force loading on the base DAD model with a bushing, and the FEM with force loading on the base DAD model with a bushing and a fastener. The FEM, experimental and theoretical curves were created the same method as previously discussed. 


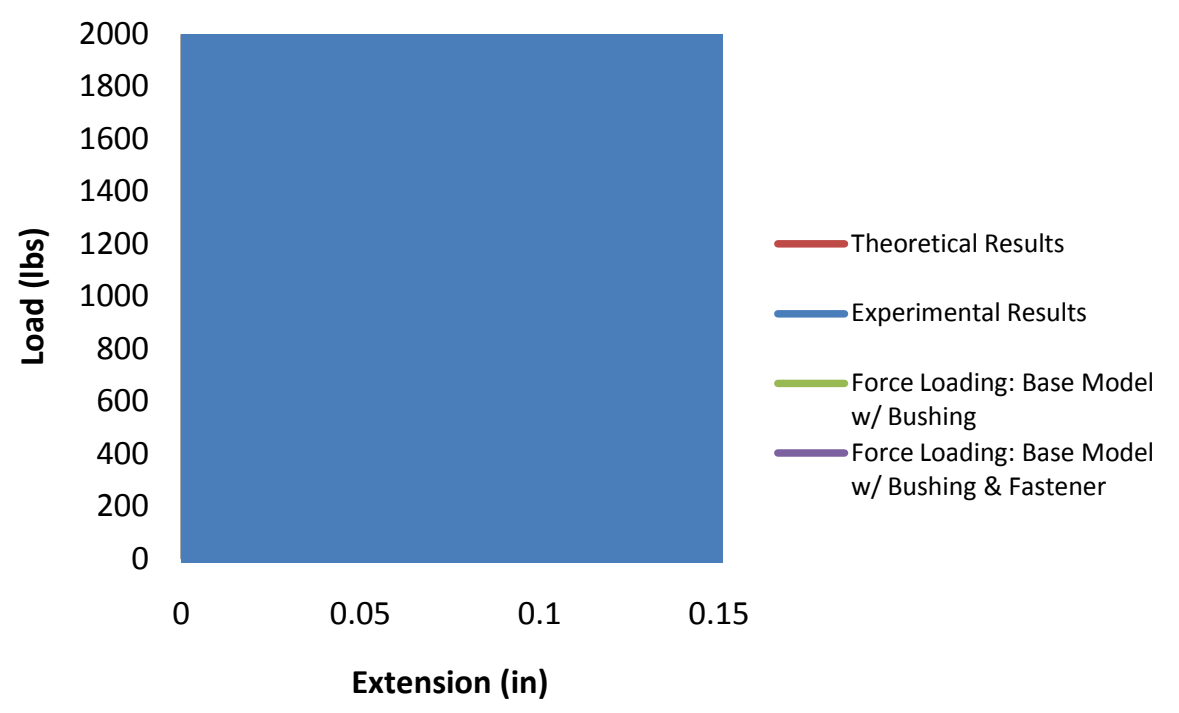

Figure 156: Load/Extension Comparison for DAD Group

The figure showed that the theoretical results over predict the elastic modulus of the experimental results and under predict the extension of the specimen. The FEM with force loading on the base DAD model with a bushing under predicts the elastic modulus of the experimental results and over predicts the extension of the specimen. The most interesting curve was the FEM with the force loading on the base DAD model with a bushing and a fastener had a similar elastic modulus of the experimental testing by overlapping one another early on, but differs halfway through the experimental results. The model did over predict the extension compared to the experimental testing, but was the closest of all FEM models. This was very surprising because that model captured the experimental results early on. The addition of a second linear curve to the model or a non-linear model would make the Fem more accurate to experimental results.

The elastic modulus of the curves can be seen in Table 35. The table showed a big contradiction to the curve by showing that the FEM with force loading on a base DAD model with a bushing and a fastener was $43 \%$ lower than the experimental values. This could be due to the elastic modulus of the experimental testing being calculated from the second slope of the 
curve rather than the first, where the FEM and the experimental results overlapped one another. The two comparisons between the experimental and FEM results showed that the FEMs needed more refinement to be accurate to the experimental values.

Table 35: Elastic Modulus Comparison for DAD Group

\begin{tabular}{|c|c|c|c|}
\cline { 2 - 4 } \multicolumn{1}{c|}{} & $\begin{array}{c}\text { Elastic } \\
\text { Modulus }\end{array}$ & $\begin{array}{c}\text { \% Difference from } \\
\text { Theoretical }\end{array}$ & $\begin{array}{c}\text { \% Difference } \\
\text { from } \\
\text { Experimental }\end{array}$ \\
\cline { 2 - 4 } & $(\mathrm{psi})$ & $(-)$ & - \\
\hline Theoretical Results & 62,650 & - & - \\
\hline Experimental Results & 59,340 & $-5.3 \%$ & $-66.0 \%$ \\
\hline $\begin{array}{c}\text { Force Loading: Base DAD } \\
\text { Model w/ Bushing }\end{array}$ & 20,168 & $-67.8 \%$ & $-43.4 \%$ \\
\hline $\begin{array}{c}\text { Force Loading: Base DAD } \\
\text { Model w/ Bushing \& } \\
\text { Fastener }\end{array}$ & 33,583 & $-46.4 \%$ & \\
\hline
\end{tabular}




\section{Conclusion}

\subsection{Summary of Thesis}

This study presented the effect of damage arrestment devices to the failure mode around a hole in a composite sandwich panel. Seven different DAD thicknesses were tested under monotonic loading. Experimental and analytical tests were performed to determine. Fatigue testing was also conducted on a control group without any DADs and a DAD group containing only a DAD thickness of three layers.

Numerical analysis was done using a finite element modeling software to predict the failure of the composite sandwich panels and compare with experimental results. Three main models were created for specimens with and without DADs. The three models were just the composite sandwich panel, a composite sandwich panel with a bushing, and a composite sandwich panel with a bushing and a fastener. Two types of loadings were applied on the six models to see which would produce the most accurate results compared to experimental testing.

The key conclusion that was determined from experimental testing and numerical analysis was summarized:

- As the DAD thickness increased in the specimen, the overall failure load increased. An addition of one DAD layer to the composite sandwich panel increased by $42.6 \%$. The overall failure load trend for the varying DAD thickness was a parabolic shape; with the maximum number of layers five for the DADs to have a failure load increase of $109.2 \%$.

- The failure mode of the experimental testing started from a pure bearing stress on specimens with small or no DAD thicknesses to a buckling failure with the face sheet delaminating for specimens with thicker DADs.

- The fatigue testing showed that the specimens with or without a DAD had similar life cycle curves and fatigue endurance limits. This showed that the DADs only strengthened the composite sandwich panel and not prolongs failure of the panels. 
- The numerical analysis showed that the type of loading on the model and the configuration of the model greatly affected the results. It was discovered that a force loading on the centerline of the specimen was ideal and that the addition of a bushing or a bushing and a fastener made the results more accurate. The addition of a bushing or a fastener helped dissipate the loading seen on the composite sandwich panel and gave it a more even distribution. The numerical analysis needs more work on it because the models only captured the elastic or linear portion of the experimental results. Better modeling of the core and the interaction between the DADs and the core must be further investigated.

\subsection{Possible Future Works}

From the experimental and numerical testing and analysis that was conducted in this research, more work must be done to continue this research to better understand the effects of damage arrestment devices around a hole of a composite sandwich panel. Since this research was a proof of concept of the usage of damage arrestment devices, this research only brushed the surface of the advantages and usage of DADs to composite structures. The following suggestions to continue the research was summarized:

- Impact and three-point bending testing must be done on the composite sandwich panels. Tension loading can also be tested.

- More fatigue testing of different DAD thicknesses. Also changing the cyclic loading seen on the specimens from a compression-compression cycle to a tension-compression cycle or a tension-tension cycle.

- A different type of DAD shape must be investigated to lessen the stress concentration seen where the DADs and the foam core meet. 
- Change the material of the face sheet to fiber glass to see if the same trends and assumptions remain the same. Changing the thickness of the face sheets can also be investigated.

- More numerical analysis on the specimens such as:

o Varying the loading applied on the test specimen that wasn't conducted in this research

o Varying the thickness of the DADs

o Creating a non-linear model for the panels to predict the plastic region 


\section{References}

' Castings, M. Effects of Manufacturing and face/core bonding on impact damage in glass/polyester-PVC foam core sandwich panels. Science Direct: Elsevier. 2008

${ }^{i i}$ Balatbat, Richard, James Dixon, Jacobson Michael, Mackey Allen, and Thomas Woo. In-Plane

Compression Response of Composite Sandwich Plate with Center Hole. Aerospace Engineering Senior Project. Aerospace Engineering Department, Cal Poly San Luis Obispo.

iii Camanho, P. P., and F. L. Matthews. "Stress Analysis and Strength Prediction of Mechanically Fastened Joints in FRP: a Review." Elsevier Science Limited (1996). Print.

iv Demelio, Giuseppe, Katia Genovese, and Carmine Pappalettere. "An Experimental Investigation of Static and Fatigue Behavior of Sandwich Composite Panels Joined by Fastener." Composites Part B. 299.308 (2001). Elsevier. Web.

${ }^{\vee}$ Song, Keun-Il, Ji-Young Choi, Jin-Hwe Kweon, Jin-Ho Choi, and Kwang-Soo Kim. "An Experimental Study of the Insert Joint Strength of Sandwich Composite Structures." Composite Structures 107.113 (2008). Elsevier. Web.

${ }^{v i}$ McCarthy, M. A., C. T. McCarthy, V. P. Lawlor, and W. F. Stanley. "Three-dimensional Finite Element Analysis of Single-bolt, Single-lap Composite Bolted Joints: Part I \& Part II." Composite Structures 140.158 (2005). Elsevier. Web.

${ }^{v i i}$ Agarwal, Bhagwan. Analysis and Performance of Fiber Composites. John Wiley \& Sons, Inc. 2006 